Monatsschr Kinderheilkd

2019 - 167 (Suppl 4):S197-S278

https://doi.org/10.1007/s00112-019-0759-4

Online publiziert: 19. August 2019

(c) Springer Medizin Verlag GmbH, ein Teil von Springer Nature 2019

\section{Abstracts des Kongresses für Kinder- und Jugendmedizin 2019}

Gemeinsame Jahrestagung der Deutschen Gesellschaft für Kinder- und Jugendmedizin (DGKJ), der Deutschen Gesellschaft für Sozialpädiatrie (DGSPJ), der Deutschen Gesellschaft für Kinderchirurgie (DGKCH), des Berufsverbandes Kinderkrankenpflege Deutschland (BeKD) und der Gesellschaft für Neuropädiatrie (GNP)

\section{1. bis 14. September 2019, München}

Wissenschaftliche Leitung

Prof. Dr. Ingeborg Krägeloh-Mann, Kongresspräsidentin DGKJ

Prof. Dr. Andreas Neu, Kongresssekretär Dr. Andreas Oberle, Kongresspräsident DGSPJ Prof. Dr. Stephan Kellnar, Kongresspräsident DGKCH Elfriede Zoller, Kongresspräsidentin BeKD

Prof. Dr. Martin Staudt, Kongresspräsident GNP 


\title{
Abstracts der 115. Jahrestagung der Deutschen Gesellschaft für Kinder- und Jugendmedizin e.V. (DGKJ)
}

\author{
Wissenschaftliche Leitung \\ Prof. Dr. Ingeborg Krägeloh-Mann, \\ Prof. Dr. Andreas Neu
}

\section{Freie Vorträge}

\section{Mehrfachbehinderte Kinder/Varia}

\section{DGKJ-FV 01 \\ Therapiezufriedenheit bei Eltern von Kindern mit frühkindlichen Fütter- und Essstörungen bei multiplen Komorbiditäten \\ Lydia Blank', Ulrike Gaiser' ${ }^{2}$ Rainer Blank ${ }^{2}$, Georg Friedrich Hoffmann ${ }^{3}$ \\ ${ }^{1}$ Heidelberg, Deutschland; ${ }^{2}$ Kinderzentrum Maulbronn gGmbH, Fachklinik für Kinderneurologie und Sozialpädiatrie, Maulbronn; ${ }^{3}$ Universitätsklinikum Heidelberg, Zentrum für Kinder- und Jugendmedizin der Universität Heidelberg, Klinik I, Heidelberg}

Fragestellung: Frühkindliche Fütter- und Essstörungen sind bei Kleinkindern mit somatischen, psychischen und entwicklungsneurologischen Störungen häufig und können sehr belastend für Bezugspersonen sein. Die vorliegende Studie thematisiert die Therapiezufriedenheit von Eltern, deren Kinder eine stationäre Esstherapie durchliefen.

Material und Methoden: 256 Eltern von zwischen 2011 und 2016 ehemals stationär behandelten Kindern ( $0-7$ Jahre) wurden mittels Fragebogen zu ihrer Einschätzung der Therapieverbesserung während und nach dem stationären Aufenthalt befragt. Es wurde die Therapiezufriedenheit allgemein sowie in den Bereichen Ernährungsverhalten, Oralmotorik, Verhaltensproblematik, Sondenernährung und außerdem die Lebenszufriedenheit (LiSat-11) sowie die familiäre Belastung ermittelt.

Ergebnisse: Unter den 103 Rückmeldungen (40 \%) wiesen $75 \%$ Entwicklungsstörungen auf, $55 \%$ waren global retardiert (40\% schwer). $20 \%$ der Kinder hatten als relevante neurologische Diagnose Zerebralparesen, 27 \% hatten psychische Störungen, davon ein Drittel autistische Störungen. Ätiologisch dominierten Frühgeburtlichkeit (48\%) sowie angeborene Fehlbildungen und Syndrome (49\%). Anamnestisch wurden $60 \%$ sondenernährt.

Bei einem Katamnesezeitraum von 6 Monaten bis 6 Jahren $(\mathrm{m}=3,1$; $\mathrm{SD}=1,8$ ) waren $61 \%$ der Eltern mit der Therapie zufrieden. Knapp die Hälfte der Eltern beobachtete während des stationären Aufenthaltes Verbesserungen, während eine Einschätzung gemäß der Befundberichte einen Zielerreichungsgrad bei $81 \%$ der Kinder aufwies. Eine langfristige Verbesserung gaben $65 \%$ der Eltern an. $77 \%$ der Eltern schilderten, dass ihr Kind bis heute kein altersentsprechendes Ernährungsverhalten aufweist. Der BMI blieb unverändert. Eltern, deren Kinder rein psychische Komorbiditäten aufwiesen, waren häufiger unzufrieden als Eltern von Kindern mit neurologischen Störungen (65 \% vs. $27 \%$ ).

Diskussion: Die vorliegende Studie weist ein Kollektiv mit unterschiedlichen Arten von frühkindlichen Fütter- und Essstörungen mit größtenteils schweren Komorbiditäten im Langzeit-Outcome auf, was die Vergleichbarkeit mit anderen Studien limitiert. Erstmals wurde hierbei die subjektive Therapie- und Lebenszufriedenheit der Eltern analysiert. Bei der Mehrzahl der Fälle konnte eine anhaltende Verbesserung des Essverhaltens erreicht werden. Die Zufriedenheit ist vor dem Hintergrund der Komplexität und Vielfalt der Komorbiditäten als eher gut zu bezeichnen. Schlussfolgerung: Die vorliegende Studie zählt zu den bisher umfangreichsten in diesem Bereich. Die erstmals untersuchten subjektiven Parameter im Umfeld der Kinder mit frühkindlichen Essstörungen sollten bei der therapeutischen Arbeit bedacht werden.

\section{DGKJ-FV 02}

PalliVac - eine multizentrische Studie zu impfprävalenten Erkrankungen in der ambulanten Palliativversorgung

Holger Hauch ${ }^{1}$, Irina Tretiakowa ${ }^{2}$, Peter Brunder ${ }^{3}$, Astrid Kimmig ${ }^{4}$, Andrea Hagenguth ${ }^{5}$, Mario Scheer ${ }^{6}$, Vera Vaillant ${ }^{1}$, Cho-Ming Chao ${ }^{7}$, Daniel Berthold ${ }^{8}$, Jens Kästner ${ }^{9}$

'UKGM Standort Gießen, KinderPalliativTeam Mittelhessen, Gießen, Deutschland; ${ }^{2}$ Klinikum Bad Hersfeld, Klinik für Kinder- und Jugendmedizin, Bad Hersfeld, Deutschland; ${ }^{3}$ Kinderpalliativteam Saarland, SAPV, Merchweiler; ${ }^{4}$ Universitätsklinikum Tübingen, Palliativteam, Tübingen; ${ }^{5}$ Deutsches Rotes Kreuz, Palliativteam, Kiel; ${ }^{6}$ Kinderhospiz Syke, Palliativteam, Syke; ${ }^{7}$ UKGM Standort Gießen, Allgemeine Pädiatrie und Neonatologie, Gießen, Deutschland; ${ }^{8}$ UKGM Standort Gießen, Klnik für Internistische Onkologie, Gießen; ${ }^{9}$ Universitätsklinikum Jena, Kinderpalliativteam, Jena

Hintergrund: In Deutschland werden Kinder und Jugendliche zunehmend häufig durch Teams der spezialisierten ambulanten Palliativversorgung (SAPV) im häuslichen Umfeld behandelt. Es handelt sich um Patienten aller Altersklassen mit verschiedenen, teilweise sehr seltenen Krankheitsbildern unklarer Prognose. Bei einem 14 Jahre alten Jugendlichen mit einer ätiologisch unklaren, schweren Entwicklungsverzögerung mit symptomatischer Epilepsie, Tetraspastik und zunehmender Skoliose sowie eingeschränkter Atemmechanik kam es im Rahmen einer Varizelleninfektion zu einer schweren Pneumonie, zunehmender Dyspnoe und leidvoller Verschlechterung des Allgemeinzustandes. Der Junge war nicht gegen Varizellen geimpft worden. In einer Literaturanalyse fanden sich bisher keine vergleichbaren Fälle. Es stellte sich die Frage, ob impfprävalente Erkrankungen (vaccine preventable diseases, VPD) in der SAPV auftreten und ob diese zu leidvollen Symptomen führen. Methodik: In einem ersten Schritt erfolgte eine Befragung aller deutschen SAPV Teams für Kinder und Jugendliche sowie aller stationären Kinderhospize. Ergebnisse: 34 Ärzte (32 SAPV- und 2 Hospizärzte) aus 12 Bundesländern beteiligten sich an der Studie. 25 Patienten mit diagnostizierter VPD (Gruppe 1) wurden gemeldet. Es wurden Infektionen mit Influenza (44\%), Varizellen $(16 \%)$ und anderen $(20 \%$ e.g. Diphtherie, Pertussis, Masern, Pneumokken und Meningokokken-Infektion) berichtet. Der Verdacht auf eine VPD (Gruppe 2) wurde bei weiteren 22 Fällen gemeldet (Influenza $56 \%$, Pertussis $14 \%$, Rotavirus $14 \%$ ). Durchschnittsalter beider Gruppen war 8,8 ( $\pm 5,7$ SD) Jahre. Verteilung der IMPaCCT Gruppen (1-4) war $5 \%$ (1), $0 \%$ (2), $55 \%$ (3), $40 \%$ (4). Die Diagnose wurde in $64 \%$ klinisch, in 
29 \% serologisch und in 59 \% mittels PCR gestellt (Mehrfachantworten waren möglich). Leidvolle Symptome (min. 1 bis max. 5 Punkte) waren Dyspnoe (3,4 $\pm 1,5 \mathrm{SD})$ und AZ-Verschlechterung (3,8 $\pm 1,1 \mathrm{SD})$. Schwere Komplikationen waren Aufnahme auf einer Intensivstation (29\%), Beatmung (20\%), Tod (6\%) sowie Infektion eines SAPV-Teammitgliedes oder Hospiz-Mitarbeiters (20\%). Die befragten Ärzte waren der Meinung, dass es hilfreich sein könnte, VPD bei Palliativpatienten zu verhindern (Mittelwert 4,4 $\pm 1,1 \mathrm{SD} ; 5=$ starke bis $1=$ keine Zustimmung).

Diskussion: Impfprävalente Erkrankungen (VPD) kommen auch in der Palliativversorgung vor. Überwiegend sind Kinder der IMPaCCT Gruppe 3 und 4 betroffen. VPD führen zu leidvollen Symptomen bis zur Intensivbehandlung, Beatmung und Tod. Weitere Untersuchungen scheinen gerechtfertigt zu sein. Der prospektive Arm der Studie PalliVac (DRKS: 00015045) wurde im Januar 2019 gestartet.

\section{Literatur \\ 1. Koch Institute $R$ (2019) recommended vaccinations in germany. https://www.rki. de/DE/Content/Kommissionen \\ 2. Hauch H et al Klin Paediat. 228(3):145-148}

\section{DGKJ-FV 03}

Mobile Musiktherapie zur Versorgung von Kindern mit schweren Hirnschädigungen und ihrer Familien im ländlichen Raum - Ein Praxisprojekt als phänomenologische Pilotstudie

\section{Brigitte Meier-Sprinz}

Zentrum für interdisziplinäre Neuropädiatrie Kempten, Musiktherapie, Kempten, Deutschland

Fragestellung: Wie muss ein ambulantes musiktherapeutisches Angebot für Familien mit Kindern mit schweren Hirnschädigungen (SHS) gestaltet sein, damit es

a) die beteiligten Eltern als hilfreich und sinnvoll wahrnehmen?

a) aus Sicht von Expert ${ }^{\star}$ innen Prozesse in Gang setzt, elterliche Kompetenzen zu fördern?

b) sich für die weitere Prozessforschung eignet?

Material und Methoden: In Kooperation mit einem neuropädiatrischen Versorgungszentrum wurde ein „home-visiting“-Konzept für Familien mit Kindern mit SHS konzipiert, durchgeführt und evaluiert. Sample: 8 Familien mit Kindern im Alter von 1,5 bis12 Jahren mit SHS (entspr. GMFCS-Level IV-V). Intervention: 7-20 Therapieeinheiten pro Familie. Datenerhebung: demographische, biographische und anamnestische Daten, aktuelle medizinische Befunde vor und nach der Intervention, Therapieverlaufsdokumentation, selektive Audio- und Videomitschnitte, fokussierte Elterninterviews. Analysemethodik: Einzelfallanalysen und vergleichende Analyse zu extrahierten Themen.

Ergebnisse: Entwicklung eines systematischen datenbankgestützten am ICF-CY orientieren Dokumentationsschemas, in einer Datensammlung werden Rahmenbedingungen und beobachtete Effekte dargestellt. Aus famlilientherapeutischer Perspektive erlaubt diese Studie keine einheitliche Aussage, da die Krankheitsbilder, die Familienkonstellationen und individuellen Bedarfe zu heterogen, die Betroffenheit und die daraus folgende psychische Belastungssituation der Familienmitglieder zu verschieden sind.

$\mathrm{Zu}$ beobachten waren in allen Familien vielfältige Impulse für die Rehabilitation, u. a.

1. zur Entwicklung von Intentionalität des geschädigten Kindes,

2. für eine profizitorientierte Kindeswahrnehmung durch die Eltern,

3. für eine Förderung der Teilhabe: Implementierung von Musik als Ausdruck von Gemeinsamkeit und Kommunikation.

Auf Seiten der Eltern war Widersprüchlichkeit und Ambivalenz zu beobachten, z. B. bei gleichzeitiger Wahrnehmung bzw. Äußerung von Hoffnung vs. Realitätswahrnehmung, Zuversicht vs. Ängsten, Insuffizienzgefühlen vs. Idealisierung.

Diskussion: Die Frage, ob (ambulante) Musiktherapie positive Effekte auf die Rehabilitation des Kindes mit SHS hat, muss noch zurückgestellt werden, da bisher die Voraussetzungen für eine kontrollierte Wirksamkeits- forschung fehlen. Diese Ergebnisse könnten zunächst als Grundlage für die Versorgungsforschung dienen, es könnten daraus manualisierte Interventionen für spezifische Neuropädiatrische Krankheitsbilder entwickelt werden.

Schlussfolgerung: Die Hypothese, dass in Familien mit Kindern mit schweren z.T. lebensbegrenzenden Hirnschädigungen der Einsatz von Musik in einem ambulanten musiktherapeutischen Angebot geeignet ist, 1.) psychosoziale Prozesse zu unterstützen und 2.) ausgehend von sich ereignenden shared moments dialogische Prozesse anzustoßen und damit die Fähigkeiten aller Beteiligten zu erweitern, konnte mit dieser Pilotstudie weiter bestätigt werden.

\section{Literatur}

1. Meier-Sprinz B, Sprinz A (2018) Musiktherapie in der neuropädiatrischen Frührehabilitation aus Sicht betroffener Eltern. Musik-, Tanz- und Kunsttherapie. Pabst Sci Publ 28(2):172-192

2. Meier-Sprinz B (2018) How Do Parents Experience M

\section{DGKJ-FV 04 \\ Methodik und Ziele der hundegestützten Therapie in der stationären Neurorehabilitation von Kindern mit schweren neurologischen Erkrankungen}

Friederike Boek', Julia Sachers', Gerhard Kluger', Evelyn Kluger', Ulrike Blankenburg ${ }^{2}$, Martina Schaudeck², Karin Hediger ${ }^{1}$

'Universität Basel, Fakultät für Psychologie, Basel, Schweiz; ${ }^{2}$ Schön Klinik Vogtareuth, Fachzentrum für pädiatrische Neurologie, Neuro-Rehabilitation und Epileptiologie, Vogtareuth, Deutschland

Hintergrund: Ergänzend zum konventionellen Behandlungsalltag, wird seit 2004 am Fachzentrum für pädiatrische Neurologie, Neuro-Rehabilitation und Epileptologie in Vogtareuth hundegestützte Therapie (HGT) durchgeführt. Ziel der vorliegenden Studie war es, diese zu evaluieren, um das das Potential von HGT in der pädiatrischen Neurorehabilitation zu untersuchen.

Fragestellung: Welche Art von Patienten nahmen an der HGT teil, wie wurde die HGT durchgeführt und in welchem Ausmaß sind die angestrebten Ziele erreicht worden?

Material und Methoden: Mithilfe der Daten von 251 Patienten, die HGT erhalten hatten, wurde eine retrospektive, explorative Analyse durchgeführt. 196 Patienten konnten eingeschlossen werden. Nebst den Charakteristika der Patienten, wurden Merkmale der durchgeführten hundegestützten Sitzungen analysiert und die vor Beginn definierten Therapieziele kategorisiert. Anhand der semistrukturierten Dokumentationsbögen, welche nach jeder hundegestützten Sitzung ausgefüllt worden waren, wurde die Zielerreichung in der HGT für jedes individuell formulierte Ziel ausgewertet.

Ergebnisse: Die Patienten $(\mathrm{N}=196)$ waren zwischen 0 und 20 Jahre alt $(M=6,48, S D=4,55) .57,7 \%$ der Patienten waren männlich, 42,3\% weiblich. Jedes Kind erhielt zwischen einer und 19 Sitzungen HGT $(M=4,3$, $\mathrm{SD}=3,2) .63,8 \%$ der Patienten hatten eine nach der Neonatalperiode erworbene Hirnschädigung, während 34,7 \% seit Geburt an einer neurologischen Erkrankung litten. 18,9 \% der Patienten erfüllten das Kriterium einer schweren Bewusstseinsstörung. 43 \% der Patienten hatten eine PEGSonde, $12,8 \%$ ein Tracheostoma und 7,6 \% eine Baclofenpumpe. $91 \%$ der 850 evaluierten Sitzungen dauerten zwischen 20 und 25 min. Es wurde lediglich ein kritischer Vorfall dokumentiert, bei dem ein Kind einen der Hunde am Fell zog. Die Inhaltsanalyse der vorab definierten Therapieziele ergab 11 Funktionsbereiche, welche es bei den Kindern zu fördern galt. Am häufigsten bezogen sich diese auf: Kontakt und Kommunikation $(\mathrm{N}=57)$, Aufmerksamkeit $(\mathrm{N}=56), \mathrm{Spa}(\mathrm{N}=51)$, Motorik $(\mathrm{N}=50)$, Entspannung $(\mathrm{N}=45)$ und soziale und emotionale Kompetenzen $(\mathrm{N}=36)$. Die Analyse der Zielerreichung zeigte, dass insgesamt $63 \%$ der vordefinierten therapeutischen Ziele in der HGT erreicht wurden. Insbesondere Ziele im sozialen, emotionalen und psychischen Bereich wurden am häufigsten erreicht (durchschnittlich $83 \%$ ), während funktionale Ziele weniger häufig umgesetzt werden konnten (durchschnittlich $39 \%$ ). 
Diskussion: Die Resultate zeigen, dass HGT mit neurologisch schwer kranken Kindern und auch mit prolongierten Bewusstseinsstörungen in der stationären Neurorehabilitation möglich ist. Sie kann ein effektiver Weg sein, besonders soziale und emotionale Kompetenzen zu fördern. Die Studie gibt somit erste Hinweise darauf, in welchen Funktionsbereichen hundegestützte Therapie als ergänzendes Behandlungsangebot für Kinder und Jugendliche mit schweren neurologischen Erkrankungen eingesetzt werden kann.

\section{DGKJ-FV 05}

Gesundheitsprobleme bei minderjährigen Flüchtlingen und Asylbewerber: Daten von zwei medizinischen Einrichtungen im Raum München

\section{Christoph Großhauser', Mathias Wendeborn², Annette Jansson ${ }^{3}$, Johannes} Hübner', Ulrich von Both', Martin Alberer

'Dr. von Haunersches Kinderspital, Klinikum der Universität München (LMU), Abteilung Pädiatrische Infektriologie, München; ${ }^{2}$ REFUDOCS, Verein zur medizinischen Versorgung von Flüchtlingen, Asylsuchenden und deren Kindern e.V., REFUDOCS, Verein zur medizinischen Versorgung von Flüchtlingen, Asylsuchenden und deren Kindern e.V., München; ${ }^{3} \mathrm{Dr}$. von Haunersches Kinderspital, Klinikum der Universität München (LMU), Abteilung Rheumatologie, München; ${ }^{4}$ Klinikum der Universität München, Ludwig-Maximilians-Universität München, Abteilung für Infektions- und Tropenmedizin, München, Deutschland

Fragestellung: Aufgrund der gewaltsamen Auseinandersetzungen in Nordafrika und dem Nahen Osten kamen 2015 und 2016 über eine Million Flüchtlinge nach Deutschland. Unbegleitete sowie begleitete minderjährige Flüchtlinge und Asylbewerber (FAs) sind dabei besonders den körperlichen und seelischen Belastungen der Flucht ausgesetzt und gehören somit zu einer überdurchschnittlich gefährdeten Gruppe von Flüchtlingen. Bezüglich des Spektrums der Erkrankungen, die bei den in Deutschland untergebrachten minderjährigen Flüchtlingen auftreten, gibt es aber bisher keine belastbaren und umfassenden Daten.

Material und Methode: Im Rahmen einer deskriptiven retrospektiven Querschnittsstudie wurden bisher knapp 1900 vorwiegend ambulante aber auch stationäre, irreversibel anonymisierte Patientenkontakte in zwei Einrichtungen im Raum München (REFUDOCS e. V. Bayernkaserne und Dr. von Haunersches Kinderspital) aus den Jahren 2015 und 2016 retrolektiv statistisch ausgewertet. Ein weiterer Datensatz mit knapp 7000 ambulanten Patientenkontakten befindet sich derzeit noch in der Auswertung. Die Datenanalyse erfolgt mit Microsoft Excel und STATA ${ }^{\circ}$.

Ergebnisse: Im Rahmen der Analyse dieser ersten Daten zeigt sich, dass sich das Spektrum der Gesundheitsprobleme minderjähriger FAs im Wesentlichen nicht vom Spektrum der in Deutschland lebenden minderjährigen Durchschnittsbevölkerung unterscheidet. Der größte Teil der Diagnosen umfasst Erkrankungen wie grippale Infekte, Atemwegsinfektionen oder Gastroenteritiden. Zudem kommt es zum gehäuften Auftreten von parasitären Erkrankungen wie Skabies. Weitere wichtige Erkrankungen bei minderjährigen FAs sind Fälle von Tuberkulose (13 Fälle bei 856 Patientenvorstellungen) und posttraumatische Belastungsstörungen (12 Fälle bei 856 Patientenvorstellungen).

Diskussion: Wie auch bei den Daten zu Erkrankungen bei erwachsenen FAs finden sich auch hier v. a. Erkrankungen des üblichen allgemeinmedizinischen Spektrums. Zudem treten Erkrankungen auf, die durch die Flucht verursacht oder begünstigt werden. Faktoren wie Herkunftsland oder Ablauf und Dauer der Flucht können dabei das Auftreten bestimmter Erkrankungen, wie z. B. der Tuberkulose, beeinflussen.

Schlussfolgerung: Die Gesamtanalyse dieses umfangreichen Patientenkollektivs kann sicher dazu beitragen, eine bessere Planung und Umsetzung der medizinischen Versorgung von minderjährigen FAs in der Zukunft zu ermöglichen.

\section{DGKJ-FV 06 \\ Antibiotikatherapie in der ambulanten Medizin: ein lokales Projekt macht Schule}

\section{Roland Tillmann', Reinhard Bornemann ${ }^{2}$}

'Kinder- und Jugendarztpraxis Roland Tillmann, Bielefeld, Kinder- und Jugendarztpraxis Roland Tillmann, Bielefeld, Bielefeld, Deutschland; 2Universität Bielefeld, Fakultät für Gesundheitswissenschaften, AG 2, Bielefeld, Deutschland

Hintergrund: Seit drei Jahren existiert das Projekt „Antibiotische Therapie in Bielefeld“ (AnTiB). Es wurde gegründet von ambulant tätigen Kinderund Jugendärzten in Bielefeld, mit dem Ziel einer rationalen Antibiotikaverordnung vor Ort. Eine rationale Therapie wird durch ein zwischen Fachkollegen, Fachrichtungen und Versorgungssektoren (Praxis, Klinik und Notfallversorgung) unterschiedliches Verschreibungsverhalten erschwert. Dieses Verhalten wird maßgeblich durch lokale Verordnungskulturen mitbeeinflusst. Dies spiegelt sich in einer bundesweit sehr inhomogenen Antibiotikaverordnungsdichte wieder, die nicht ohne weiteres epidemiologisch erklärt werden kann.

Fragestellungen: Wie können in einem lokalen Projekt innerhalb der pädiatrischen Fachgruppe möglichst viele Kolleginnen und Kollegen adressiert, einbezogen und mitgenommen werden, um zu einer rationalen Antibiotikaverordnungskultur zu entwickeln? Wie können die verschiedenen Versorgungssektoren vor Ort - Praxen, KV-Notfallversorgung und Klinik - einbezogen werden? Wie kann dies mit benachbarten Regionen ausgetauscht und flächendeckend implementiert werden?

Methoden: In einem strukturierten Vernetzungs- und Kommunikationsprozess werden auf lokaler Ebene zunächst fachgruppenintern alle Beteiligten bei der Erstellung von Empfehlungen für die ambulante antibiotische Therapie einbezogen. Das Ergebnis wird formell verabschiedet und in die Praxis umgesetzt. Der Prozess wird auf weitere Fachgruppen und auch überregional angewendet.

Ergebnisse: In Bielefeld wurden nach dieser Methodik 1/2017 Empfehlungen zur ambulanten Antibiotikabehandlung erarbeitet und inzwischen bereits 1/2018 überarbeitet. Das Konzept kann in Bielefeld als erfolgreich in der ambulanten Pädiatrie eingeführt betrachtet werden. Inzwischen wurde es auch in der ambulanten Frauenheilkunde und Hausarztmedizin etabliert, weitere Fachrichtungen stehen bevor. Aus dieser Zusammenarbeit vor Ort entwickelte sich das interdisziplinäre und sektorübergreifende regionale „ABS-Netzwerk Bielefeld - Ostwestfalen-Lippe“. Das pädiatrische Grundkonzept konnte bereits erfolgreich auch überregional transferiert werden: Ostwestfalen, Lippe, Lübeck, Augsburg u. a. Eine bundesweite Programmatik befindet sich in Vorbereitung.

Schlussfolgerungen: In der ABS-Programmatik für die ambulante Medizin sollten berücksichtigt werden, dass Antibiotikaverordnungen in einem sozialen Kontext stattfinden. Die Beschränkung auf abstrakte Leitlinien bzw. Schulungsmaßnahmen für einzelne Ärzte bzw. Praxen ist vermutlich nicht ausreichend effektiv. Die Effektivität der eigenen Maßnahmen wird durch eine Analyse der Antibiotikaverordnungsdaten der Jahre 2015-2018 derzeit evaluiert, erste Ergebnisse werden auf der Tagung berichtet. 
DGKJ-FV 08

EKG - Screening in der ersten Lebenswoche - Erfahrungen aus der KUNO-Kids-Geburtskohortenstudie

Stephan Gerling ', Alexander Simma², Antonia Potapow ${ }^{3}$, Stephan Döring', Susanne Brandstetter', Michael Melter', Birgit Seelbach-Göbel', Christian Apfelbacher ${ }^{6}$, Michael Kabesch ${ }^{7}$, Holger Michel ${ }^{7}$

'Universität Regensburg (KUNO-Kliniken), Klinik St. Hedwig, Regensburg, Kinderkardiologie, Regensburg, Deutschland; ${ }^{2}$ Universitätsklinik Tübingen, Kinder- und Jugendmedizin, Tübingen; ${ }^{3}$ Universität Regensburg, Medizinische Fakultät, Regensburg; ${ }^{4}$ Universität Regensburg (KUNOKliniken), Klinik St. Hedwig, Regensburg, Kinder- und Jugendmedizin, Regensburg; ${ }^{5}$ Klinik für Gynäkologie und Geburtshilfe, Klinik St. Hedwig, Krankenhaus Barmherzige Brüder, Lehrstuhl für Geburtshilfe der Universität Regensburg, Regensburg, Gynäkologie und Geburtshilfe, Regensburg; ${ }^{6}$ Universität Regensburg, Regensburg, Institut für Epidemiologie und Präventivmedizin, Medizinische Soziologie, Universität Regensburg, Regensburg, Regensburg; ${ }^{7}$ Universität Regensburg (KUNO-Kliniken), Klinik St. Hedwig, Regensburg, Abteilung für Pneumologie, Regensburg

Hintergrund: Mit einer Prävalenz von 1:

2000-2500 ist das kongenitale Long QT-Syndrom (LQTS) häufiger als bisher angenommen. Es wird vermutet, dass ein nicht unerheblicher Teil der Fälle von plötzlichem Kindstod Folge einer verlängerten QT-Zeit und der dadurch begünstigten kardialen Arrhythmien sein könnte. Eine Reihe von Studien untersuchte den potentiellen Nutzen und die Durchführbarkeit eines EKG-Screenings im Neugeborenen-Alter. Thematisiert wurden die Kosten-Nutzen-Relation, die Genauigkeit der EKG-Interpretation sowie der ideale Ableitungszeitpunkt. In der vorliegenden Studie wurde eine EKG-Screening bei Neugeborenen in der ersten Lebenswoche evaluiert. Methoden: Im Rahmen unserer prospektiven Geburtskohortenstudie, der KUNO-Kids-Gesundheitsstudie, Klinik St. Hedwig in Regensburg, wurde bei Neugeborenen in der ersten Lebenswoche ein 12-Kanal-EKG abgeleitet. Eine ausführliche Anamnese bezüglich familiärer Herzerkrankungen, mütterlicher Medikation, etc. wurde erhoben. Die Auswertung der EKGs erfolgte durch Kinderkardiologen. Weiterführende kardiologische Untersuchungen wurden in Abhängigkeit von EKG-Veränderungen veranlasst. Die QT-Zeit wurde händisch gemessen und die Formel nach Bazett zur Frequenz-Korrektur (QTc) verwendet. Bei QTc-Werten über $450 \mathrm{~ms}$ wurde ein Kontroll-EKG vor Entlassung und/oder nach 3-4 Wochen durchgeführt. Probanden deren initiale QTc über $500 \mathrm{~ms} \mathrm{lag,} \mathrm{wurden} \mathrm{stationär}$ überwacht, bis es zu einer Normalisierung der QTc kam, oder eine antiadrenerge Therapie eingeleitet worden war.

Ergebnisse: Bedrohliche Herzerkrankungen fanden sich bei 4 von 2251 Probanden. Die mittlere QTc betrug $414 \mathrm{~ms}$ (SD 24,5 ms). Bei 99 Probanden $(4,4 \%)$ war die initiale QTc verlängert (>450 ms) und bei 80 EKGs war die QT-Zeit initial nicht messbar. Daher wurden bei 8,3\% der Teilnehmer ein Kontroll-EKG und bei 1,2 \% ein zweites Kontroll-EKG durchgeführt. In diesen nahm die QTc von 482 auf 426 ms im Mittel ab. Nach 4 Wochen zeigten nur noch zwei Probanden eine QTc $>450 \mathrm{~ms}$. In beiden Fällen konnte molekulargenetisch eine KCNQ1-Mutation nachgewiesen werden, die als ursächlich für ein LQTS Typ I bekannt ist. Durch ein Kaskaden-Screening konnten in beiden Familien mehrere Mutationsträger erkannt, beraten und behandelt werden. Als weitere relevante Diagnosen fanden sich ein WPW-Syndrom, sowie ein HLH-Syndrom, welches zum Zeitpunkt der EKG-Ableitung noch nicht klinisch auffällig geworden war. Zusammenfassung: Bei Neugeborenen kann mittels eines 12-Kanal-EKGs in der ersten Lebenswoche, sowohl ein LQT-Syndrom aber auch weitere bedrohliche Herzerkrankungen erkannt werden. Der frühe Ableitungszeitpunk führt zu einer höheren Rate an falsch-positiven Ergebnissen, aber potentiell lebensbedrohliche Erkrankungen können so noch vor der Entlassung des Kindes aus der Geburtsklinik diagnostiziert und eine Therapie umgehend eingeleitet werden.

\section{DGKJ-FV 09 \\ Juvenile Granulosazelltumore - Bericht der MAKEI- und STEP- Register}

Andrea Witowski ' , Felicitas Hippert ${ }^{2}$, Benedikt Bernbeck ${ }^{2}$, Alfred Längler ${ }^{3}$, Christian Vokuhl', Gabriele Calaminus ${ }^{5}$, Ulrich Göbel ${ }^{6}$, Dominik Thomas Schneider ${ }^{2}$

${ }^{1}$ Witten, Deutschland; ${ }^{2}$ Klinikum Dortmund Klinikzentrum Mitte, Pädiatrie, Dortmund; ${ }^{3}$ Universität Witten/Herdecke, Gemeinschaftskrankenhaus Herdecke, Pädiatrie, Witten; ${ }^{4}$ Institut für Pathologie des Universitätsklinikum Schleswig-Holstein, Campus Kiel, Sektion für Kinderpathologie, Kiel; ${ }^{5}$ Universitätskinderklinik Bonn, Pädiatrische Hämatologie/Onkologie, Bonn; ${ }^{6}$ Heinrich-Heine-Universität Düsseldorf, ESPED Büro, Düsseldorf

Hintergrund: Die juvenilen Granulosazelltumoren (juvGCT) gehören zu den seltenen Keimstrang-Stromatumoren und machen ca. $5 \%$ aller pädiatrischen Ovarialtumoren aus. Der Großteil der juvGCT wird in einem frühen Stadium diagnostiziert und ist prognostisch günstig. In historischen Kohorten liegt das ereignisfreie Überleben (EFS) bei gut $85 \%$. Allerdings sinken bei einer präoperativen Tumorruptur, peritonealen Aussaat oder hohen Mitoserate die Überlebenschancen unter 50 \%. Daher wurde durch die MAKEI Arbeitsgruppe eine Therapiestratifizierung entwickelt, anhand derer Patientinnen mit erhöhtem Rezidivrisiko für eine adjuvante Chemotherapie ausgewählt werden. In dieser Zwischenanalyse des Registers für Seltene Tumorerkrankungen in der Pädiatrie (STEP) soll diese Stratifizierung evaluiert werden.

Material und Methode: Zwischen 2001 und 2018 wurden 83 Patientinnen (Medianes Alter 9,9 Jahre) mit einem juvGCT prospektiv im MAKEI bzw. STEP Register erfasst. Für die Register liegen positive Voten der Ethikkommissionen Düsseldorf und Erlangen vor. Nach Aufklärung und Einwilligung in die Datenerfassung wurden die Daten anhand von Meldebögen, Operationsberichten und pathologischen Befunden (einschl. Referenzpathologie) zentral ausgewertet. Die histopathologische Klassifikation erfolgte nach WHO, das Staging entsprechend FIGO Klassifikation.

Ergebnisse: Die typischen klinischen Erstsymptome umfassten Bauchschmerzen, eine Bauchumfangszunahme und endokrinologische Symptome wie eine Pseudopubertas praecox oder eine sekundäre Amenorrhoe. Der maximale Tumordurchmesser betrug im Median $11 \mathrm{~cm}(2-30 \mathrm{~cm})$. Die Stadienverteilung nach FIGO ergab: Ia/b 48 Patientinnen, Ic 31 Patientinnen, II/III 4 Patientinnen. Die Therapie beinhaltete eine Ovarektomie bzw. Adnektomie. Patientinnen im Stadium Ia wurden nachbeobachtet. Folgende Patientinnen wurden für eine adjuvante cisplatinhaltige Chemotherapie ausgewählt: Stadium Ic mit intraoperativer Ruptur: 1/14 Pat.; Ic nach präoperativer Ruptur: 11/17 Pat., sowie bei alle vier Pat. ab Stadium II. Unter diesen Patientinnen - mit in historischen Kohorten ungünstiger Prognose - wurden keine Rezidive beobachtet.

Nach einer medianen Nachbeobachtungszeit von 63 Monaten wurden 3 Ereignisse und ein Todesfall berichtet. Alle Rezidivtumoren waren primär im Stadium Ia und zeigten eine hohe Proliferationsrate. Zwei Patientinnen konnten mit intensiver Chemotherapie in eine anhaltende Zweitremission gebracht werden. Im Rahmen dieser Zwischenanalyse liegt das EFS bei $0,95 \pm 0,026(80 / 83)$ und das Gesamtüberleben bei 0,98 $\pm 0,02(82 / 83)$. Schlussfolgerung: Durch die zentrale Referenzdiagnostik sowie den Aufbau von Beratungsstrukturen für die Behandlung von Patientinnen mit juvGCT konnte die Prognose im Vergleich zu historischen Gruppen verbessert werden. Entscheidend hierfür war die Einführung einer risikostratifizierten adjuvanten Chemotherapie ab einem Tumorstadium FIGO Ic mit präoperativer Tumorruptur.

\section{DGKJ-FV 10 \\ Ethische Konflikte in der Kindermedizin in Zeiten der Ökonomisierung.}

Annic Weyersberg', Ursula Köstler², Christiane Woopen ${ }^{3}$

'Uniklinik Köln, Universität zu Köln, Forschungsstelle Ethik/ceres/

Kinderklinik, Köln, Deutschland; ${ }^{2}$ Universität zu Köln, ceres, Köln,

Deutschland; ${ }^{3}$ Uniklinik Köln, Universität zu Köln, ceres, Köln, Deutschland 
Fragestellung: Fehlanreize und Unterfinanzierung in Zeiten der Ökonomisierung führen dazu, dass die Pädiatrie unter einem besonderen ökonomischen Druck steht. Dies birgt gerade bei einer vulnerablen Patientengruppe wie derjenigen der Kinder ein besonderes Konfliktpotential. Ziel der Studie ist eine erste empirische Erhebung und Analyse ökonomisch bedingter Konflikte in der Pädiatrie.

Material und Methode: Im Rahmen eines etablierten, qualitativen Studiendesigns wurden 31 Einzel- und 6 Gruppeninterviews mit 50 Stakeholdern der stationären klinischen Versorgung der Pädiatrie und Kinderchirurgie in ganz Deutschland durchgeführt.

Ergebnisse: Nahezu alle Interviewteilnehmer schilderten ein deutliches Konfliktpotential durch ökonomisch motivierte Konflikte in der Pädiatrie. Art und Ausprägung unterschieden sich abhängig von Determinanten wie der Berufsgruppe, der Position und der Trägerschaft des Krankenhauses. Die Befragten kritisieren eine Leistungsverdichtung durch gestiegene $\mathrm{Pa}$ tientenzahlen, schwerere und komplexere Krankheitsbilder, kürzere Aufenthaltsdauern, erlösrelevante Dokumentationsaufgaben und zeitaufwändige Mangelverwaltung. Das Fach Pädiatrie erfahre eine Entwertung und zunehmende Abhängigkeit von anderen Fachbereichen, Drittmitteln und Spenden

Der Zugang zu Gesundheitsleistungen und die Versorgungsqualität von Kindern seien eingeschränkt. Besonders betroffen sind chronisch und schwer kranke Kinder. Fehl- und Unterversorgung mit kritischen Versorgungsengpässen, verdeckte Rationierung, die Notwendigkeit der Patientenselektion, fachliche und juristische Verantwortung bei fehlender Gestaltungsfreiheit und zahlreiche weitere in den Interviews beschriebene Konflikte führen zu erheblichen ethischen Konflikten und Dilemmata. Diskussion: Die geschilderten Folgen von Unterfinanzierung, erlösorientierter Umstrukturierung, Personal- und Ressourcenmangel führen zu Ungleichbehandlung und kritischer Unterversorgung in der Pädiatrie, v. a. für chronisch und schwer kranke Kinder. Eine flächendeckende Versorgung und Zugangsgerechtigkeit von Kindern zu Gesundheitsleistungen scheint nicht mehr gewährleistet. Fraglich ist, in wieweit den Verpflichtungen aus der UN-Kinderrechts-Konvention ausreichend nachgekommen wird.

Schlussfolgerung: Die Studie stellt eine erste wissenschaftliche Grundlage zu den Folgen der Ökonomisierung in der Pädiatrie dar und erlaubt damit eine wissenschaftliche Einordnung der Problematik. Die Ergebnisse verweisen klar auf strukturelle Fehlentwicklungen und spezifische Versorgungsdefizite in der pädiatrischen Versorgung in Deutschland, insbesondere für die Gruppe der chronisch und schwer kranken Kinder. Im Hinblick auf die besonderen Rechte des Kindes besteht damit weiterer Forschungsbedarf zur Quantifizierung der Problematik, sowie Handlungsbedarf für die an der Gesundheitsversorgung beteiligten Akteure und Institutionen.

\section{Epidemiologie}

\section{DGKJ-FV 11 \\ Longitudinales Wachstum zwischen der frühen Kindheit und Jugendalter in Abhängigkeit vom Gestationsalters}

Juliane Spiegler', Marina Mendonca², Dieter Wolke²

'Universitätsklinikum Schleswig-Holstein, Neuropädiatrie, Lübeck, Deutschland; ${ }^{2}$ University of Warwick, Department of Psychology and Division of Mental Health \& Wellbeing, Coventry, UK

Fragestellung: Frühgeborene sind häufig kleiner und zarter in der Kindheit als Gleichaltrige. Vergleiche des Wachstums zwischen den verschiedenen Gestationsaltersgruppen sind rar. Ein Aufholwachstum wird bis zum Jugendalter angenommen, aber aktuelle longitudinale Daten fehlen. Methoden: Wir analysierten longitudinale Wachstumsdaten (Körpergröße, Body mass index und Alter bei Menarche) im Alter von 3, 5, 6-8 und 14-16 Jahren in der Millennium Cohort Study aus dem Vereinigten Königreich zusammen mit Daten von extrem unreifen Frühgeborenen aus Schleswig-Holstein eines vergleichbaren Geburtsjahrganges. Das Wachstum von extrem unreifen $(\mathrm{N}=100)$, sehr unreifen $(\mathrm{N}=86)$, moderat unreifen $(\mathrm{N}=127)$ und späten Frühgeborenen $(\mathrm{N}=609)$ wurde mit Reifgeborenen $(\mathrm{N}=10.125)$ verglichen bei gleichzeitiger Kontrolle von bekannten Einflussfaktoren (Geschlecht, SDS des Geburtsgewichtes, elterliche Bildung, maternale Größe bzw. Body mass index).

Ergebnisse: Unterschiede in der Körperlänge waren zwischen der frühen Kindheit und dem Jugendalter stabil. Extrem unreife Frühgeborene waren ca. $4 \mathrm{~cm}$, sehr unreife Frühgeborene ca. $2 \mathrm{~cm}$, moderat Frühgeborene ca. $1,5 \mathrm{~cm}$ und späte Frühgeborene ca $1 \mathrm{~cm}$ kleiner als Reifgeborene. Der Body mass index war geringer, je unreifer die Kinder geboren waren. Das Alter bei Eintritt der Menarche unterschied sich nicht in den verschiedenen Gestationsaltersgruppen.

Diskussion: Das Wachstum von extrem unreifen und sehr unreifen Frühgeborene unterscheidet sich auch im Jugendalter noch von Reifgeborenen. Veränderungen des neonatalen Managements in den letzten 10 bis 20 Jahren hat die Unterschiede in der Körperlänge von extrem und sehr unreifen Frühgeborenen im Vergleich zu Reifgeborenen nicht verringert.

\section{DGKJ-FV 12}

Inzidenz der Neugeborenenseptikämien durch Streptokokken der Gruppe B und Escherichia Coli: Analyse von Krankenversicherungsdaten der Barmer GEK von 2005 bis 2017

Anna-Lisa Sorg', Mathias Klemme², Rüdiger von Kries ${ }^{3}$, Viola Obermeier ${ }^{3}$ 'Institut für soziale Pädiatrie und Jugendmedizin, Epidemiologie, München, Deutschland; ${ }^{2}$ Klinikum der Universität München, Ludwig-MaximiliansUniversität München, Abteilung Neonatologie, München; ${ }^{3}$ Institut für soziale Pädiatrie und Jugendmedizin, Ludwig-Maximilians-Universität München, Abteilung Epidemiologie, München

Fragestellungen:

1. Hat das präpartale Screening der Mütter auf Streptokokken der Gruppe B (GBS) einen Einfluss auf die Inzidenz der Neugeborenensepsis in Deutschland von 2005 bis 2017 ?

2. Wie hat sich der Anteil an Frauen mit bekannter GBS Besiedelung im gleichen Zeitraum verändert?

3. Garantiert ein bekannter mütterlicher GBS Status die Vermeidung der EOS?

4. Welchen Einfluss haben das Screening und die intrapartale Antibiotikaprophylaxe (IAP) auf die Inzidenz der Neugeborenensepsis durch E. Coli?

Methodik: Datengrundlage bilden die Krankenversicherungsdaten der Barmer GEK. Es wurden jährliche Mutter-Kind-Kohorten gebildet, bei denen Mutter und Neugeborenes eindeutig zugeordnet werden konnten $(\mathrm{N}=406.279)$. Die Ermittlung der Neugeborenensepsis durch GBS und E. Coli erfolgte über die ICD P36.0 und P36.4. Bei der GBS Sepsis wurde zwischen der frühen Form EOS (Early-Onset) und der späten Form LOS 
(Late-Onset) unterschieden. Als LOS wurden Neugeboren definiert, die nach der Geburt entlassen, mit der ICD P36.0 wiederaufgenommen wurden und bei Wiederaufnahme älter als 3 Tage waren. Die Besiedelung der Mutter mit GBS wurde durch die ICD B95.1 ermittelt. Bei Müttern ohne B95.1 wurde der GBS Status als unbekannt definiert.

Ergebnisse: Die Inzidenz der EOS nahm von 1,5 pro 1000 Geburten [95\%KI 1,2-1,9] im Jahr 2005 auf 0,3 pro 1000 Geburten [95 \%KI 0,10,6] im Jahr 2017 signifikant ab. Die Inzidenz der LOS lag im Mittel bei 0,1 pro 1000 Geburten [Range $0-0,3$ ].

Der Anteil an Frauen mit positivem GBS Befund lag 2005 bei 11 von 1000 Frauen [ $95 \% \mathrm{KI} 10-12$ ] und stieg bis 2017 auf 42 von 1000 Frauen [95 \%KI $40-45]$ an.

Die Inzidenz der EOS mit unbekannter mütterlicher GBS Besiedelung nahm signifikant ab [2005: 1,4 pro 1000; 2017: 0,2 pro 1000]. Der Anteil der Neugeborenen, die trotz bekanntem positivem GBS Status der Mutter erkrankten, änderte sich kaum und lag bei 0,2 pro 1000 Geburten. Die Inzidenz der Neugeborenensepsis durch E. Coli schwankte um den Mittelwert 0,5 pro 1000 Geburten [Range $0,1-0,6$ ].

Diskussion: Basierend auf Versicherungsdaten nimmt die Inzidenz der GBS Neugeborenensepsis seit 2005 ab. Eine Erklärung könnte die zunehmende Inanspruchnahme des Screenings und der IAP sein. Diese Annahme wird durch die Zunahme des Anteils der Mütter mit bekannter GBS Besiedelung bekräftigt. Ob der Verlauf der B95.1 tatsächlich die Abstrichhäufigkeit abbildet, wird in einer nachfolgenden Studie überprüft.

Eine weitere interessante Fragestellung ist, warum Kinder erkrankten, obwohl der positive GBS Status der Mutter bekannt war. Möglicherweise besteht ein Verbesserungsbedarf bei der Durchführung der IAP bei positiven Screening in der klinischen Praxis.

Schlussfolgerung: Das GBS Screening als IGEL-Leistung scheint zunehmend mehr angenommen worden zu sein. Zeitgleich ist die Inzidenz der EOS mit unbekanntem Expositionsstatus signifikant zurückgegangen. Auf Grundlage dieser Daten sollte der GBA die Frage nach der Aufnahme des Screenings als Regelleistung erneut diskutieren.

\section{DGKJ-FV 13}

\section{Einflussfaktoren auf den Schweregrad virusassoziierter akuter respiratorischer Erkrankungen (ARE) bei Kindern im Alter von 1-5 Jahren im Zeitraum von Januar-April der Jahre 2013 und 2014 in Bayern}

Katrin Hartmann', Andrea Streng', Miriam Härlein', Christiane Prifert', Benedikt Weißbrich'2, Johannes G. Liese

${ }^{1}$ Kinderklinik und Poliklinik, Universitätsklinikum Würzburg, Infektionsepidemiologie, Würzburg, Deutschland; ${ }^{2}$ Universität Würzburg, Institut für Virologie und Immunbiologie, Würzburg, Deutschland

Fragestellung: Virusassoziierte ARE tragen weltweit den größten Anteil zur Krankheitslast von Kindern bei. Das Krankheitsbild variiert von der leichten Infektion der oberen Atemwege bis zur schweren intensivpflichtigen Pneumonie. Ziel dieser Arbeit war die Darstellung von Einflussfaktoren auf den Schweregrad viraler ARE bei Kleinkindern.

Material und Methode: Die Datengrundlage bildeten drei unabhängige epidemiologische Surveillance-Studien mit vergleichbarer Erfassung klinischer Daten von pädiatrischen ARE-Patienten aus der ambulanten ( $n=759)$, stationären $(n=960)$ bzw. intensivstationären $(n=536)$ Versorgung in Bayern. Bei allen Patienten erfolgte die virologische Diagnostik aus Nasen-/Rachenabstrich mittels multiplex PCR (FTD ${ }^{\circledR}$ Respiratory pathogens 21 ), mit Testung auf Adeno-, hum. Boca-, Corona-, Entero-, Influenza-, hum. Metapneumo-, Parainfluenza-, Parecho-, Respiratorisches Synzytial- (RSV) sowie Rhinovirus. In die vorliegende Analyse wurden Kinder derselben Altersgruppe (1-5 Jahre) und demselben Erhebungszeitraum (Januar-April 2013/-14) eingeschlossen. Die ARE-Patienten wurden mit Hilfe eines studienübergreifenden Scores, der klinische Diagnosen und Therapien berücksichtigte, als leicht, mittelschwer und schwer erkrankt definiert. Die potentiellen Einflussfaktoren wurden sowohl deskriptiv als auch mittels ordinaler logistischer Regression analysiert.
Ergebnisse: Insgesamt lagen Daten zu 838 Kindern, 50 (6\%) aus intensivstationärer, $235(28 \%)$ aus stationärer und $553(66 \%)$ aus ambulanter Versorgung, vor. 87 (10\%) der Kinder waren schwer, 360 (43\%) mittelschwer und 391 (47\%) leicht erkrankt. Kinder mit schweren Krankheitsverläufen waren im Median jünger (2,0 im Vgl. zu 2,5 bzw. 3,2 Jahren), häufiger untergewichtig bei einem BMI $<10$. Perzentile $(28,8 \%$ im Vgl. zu $24,1 \%$ bzw. $15,8 \%)$ und litten vermehrt unter chronischen Vorerkrankungen $(66,7 \%$ im Vgl. zu 29,4 \% bzw. 12,3 \%) als Kinder mit mittelschwerer bzw. leichter ARE. Hinsichtlich der viralen Erregern war nur ein RSV-Nachweis mit einem höheren Schweregrad assoziiert (Odds Ratio (OR) 3,1; Konfidenzintervall (KI) 2,0-4,6 für RSV-Monoinfektionen). In der multifaktoriellen Regressionsanalyse war das Vorliegen einer chronischen Vorerkrankung mit einer OR von 4,4 (KI 3,1-6,1) der größte Einflussfaktor auf den Schweregrad der ARE.

Schlussfolgerung: Patientencharakteristika wie Alter, BMI und Vorerkrankungen hatten einen signifikanten Einfluss auf den Schweregrad von ARE bei Kleinkindern. Eine Ausnahme bildete lediglich RSV; sein Nachweis war deutlich mit schwereren Krankheitsverläufen assoziiert. Die Ergebnisse unterstreichen den hohen Bedarf von präventiven Maßnahmen, wie beispielsweise einer aktiven Immunisierung zur Vermeidung einer RSVErkrankung.

\section{DGKJ-FV 14}

20 Jahre Neugeborenen-Screening in Bayern, 10 Jahre flächendeckendes Hörscreening: Ergebnisse-Herausforderungen-

\section{Perspektiven}

\section{Inken Brockow', Bernhard Liebl', Anja Lüders³, Uta Nennstiel'}

'Bayerisches Landesamt für Gesundheit und Lebensmittelsicherheit (LGL), GE4 Screeningzentrum, München-Oberschleißheim, Deutschland; ${ }^{2}$ Bayerisches Landesamt für Gesundheit und Lebensmittelsicherheit (LGL), Bereichsleiter AP, GE, R, München-Oberschleißheim, Deutschland; ${ }^{3}$ Bayerisches Landesamt für Gesundheit und Lebensmittelsicherheit (LGL), GE4, München-Oberschleißheim

Hintergrund: Seit 20 Jahren wird in Bayern das Neugeborenen-Screening (NGS) im Rahmen eines Screening-Programms durchgeführt. Dies umfasst zusätzlich die Sicherstellung der Vollständigkeit des NGS, die Erinnerung an notwendige Kontrolluntersuchungen bei auffälligem Screening (Tracking), sowie die Bereitstellung von Dokumentationsbögen für die Rückmeldungen der Ergebnisse der Kontrolluntersuchungen durch das LGL. Alle Beteiligten des Screeningprozesses werden frühzeitig eingebunden. Wichtige Erkenntnisse aus 20 Jahren Evaluation des NGS in Bayern sollen hier dargestellt werden.

Material und Methoden: Die bayerischen Screeningdaten und die Ergebnisse einer nach auffälligem Screening notwendigen weiteren Kontrolluntersuchung werden nach Zustimmung der Eltern an das LGL übermittelt. Zur Sicherung einer hohen Prozessqualität des Screening-Programms erfolgt eine kontinuierliche Evaluation der Daten.

Ergebnisse: Seit der Einführung des NGS wurden $99 \%$ der bayerischen Neugeborenen gescreent, bis Ende 2017 waren das 2.107.864 Kinder. Dabei wurden 1759 Kinder mit einer der Zielkrankheiten entdeckt. Ein Hörscreening wurde bei 1.026.433 Neugeborenen durchgeführt, bei 1029 Kindern wurde eine beidseitige, permanente Hörstörung im Median von 4,7 Monaten diagnostiziert. Für eine frühe Diagnosestellung war bei etwa 40 \% dieser Kinder ein Tracking des LGL notwendig. Seit September 2016 wurde bei 64 Kindern durch Erweiterung des NGS eine Mukoviszidose (CF) diagnostiziert. Nur bei 1,5\% der Kinder mit positivem CF-Screening wurde keine weitere Abklärung dokumentiert.

Diskussion: Das NGS ist eine sehr erfolgreiche Maßnahme der Sekundärprävention. Die hohe Vollständigkeit des Screenings und sehr gute Prozessqualität in Bayern kann nur durch ein Trackingsystem des bayerischen Öffentlichen Gesundheitsdienstes mit Beteiligung des LGL und der Gesundheitsämter erreicht werden. Ein Tracking der notwendigen Kontrolluntersuchungen einschließlich der Rückmeldung der Ergebnisse ist insbesondere beim Hörscreening sehr wichtig, um eine frühe Diagnosestellung und eine niedrige lost to follow-up Rate zu erreichen. Die Erweiterung des 
NGS auf neue Zielkrankheiten, wie z. B. im September 2016 auf CF oder in Zukunft auf schwere Immundefekte (SCID) stellt neue Herausforderungen. Bewährt hat sich die frühe Einbindung aller Beteiligten des Screeningprozesses und die Erstellung von Dokumentationsbögen für die einfache und zuverlässige Rückmeldungen der Ergebnisse der Kontrolluntersuchungen, um eine möglichst niedrige lost to follow-up Rate zu erreichen. Schlussfolgerung: Für die Sicherstellung einer guten Prozessqualität beim Screening ist ein Trackingsystem zur Sicherstellung der Vollständigkeit und zeitnahen Durchführung der bei einem auffälligen Screening notwendigen Kontrolluntersuchung, einschließlich der Rückmeldung der Ergebnisse, notwendig. Der Screeningprozess sollte regelmäßig, insbesondere bei Einführung neuer Zielkrankheiten, evaluiert werden.

\section{DGKJ-FV 15}

\section{Arzneimittelbedingte stationäre Aufnahmen bei Kindern und} Jugendlichen - Was ist vermeidbar?

Irmgard Toni, Stefan Wimmer, Regina Trollmann, Wolfgang Rascher, Antje Neubert

Universitätsklinikum Erlangen, Kinder- und Jugendklinik, Erlangen, Deutschland

Hintergrund: Aufgrund des hohen off-label Gebrauchs (etwa 46-64\% der Patienten im ambulanten Bereich), fehlenden pädiatrischen Darreichungsformen und komplexen Dosierungsberechnungen treten Unerwünschte Arzneimittelwirkungen (UAW) und Medikationsfehler (MF) bei Kindern häufiger als bei Erwachsenen auf [1-3]. 3 bis $5 \%$ der Krankenhausaufnahmen in der Kinder- und Jugendmedizin sind arzneimittelbedingt [3-5].

Das Ziel der vorliegenden Studie war es, Charakteristika und Vermeidbarkeit von arzneimittelbedingten stationären Aufnahmen in der Pädiatrie zu untersuchen.

Methode: In der Kinder- und Jugendklinik Erlangen wurde eine 10-monatige Beobachtungsstudie durchgeführt. Alle Patienten zwischen 0 und $<18$ Jahren, die im Studienzeitraum aufgenommen und mindestens $24 \mathrm{~h}$ auf einer allgemein-pädiatrischen Station behandelt wurden, erfüllten die Einschlusskriterien und wurden auf UAW und MF als Grund ihrer stationären Aufnahme gescreent.

UAW und MF wurden durch intensive Aktendurchsicht (sog. Chart-Review) identifiziert und der mögliche Zusammenhang zwischen Arzneimittelanwendung und Krankenhausaufenthalt standardisiert bewertet.

Ergebnisse: 741 Patienten wurden für die Analyse und Bewertung berücksichtigt. Das mediane Alter der Patienten betrug 7 Jahre (IQR 1-13), die Aufenthaltsdauer 2 Tage (IQR 2-4). 55 Ereignisse wurden im Zusammenhang mit einer Arzneimittelanwendung vor dem stationären Aufenthalt identifiziert. 44 (80,0 \%) dieser Ereignisse führten zur stationären Behandlung und wurden somit als schwerwiegend klassifiziert. Die Inzidenz der arzneimittelbedingten stationären Aufnahmen betrug 5,9\%. 21 (47,7 \%) Ereignisse waren vermeidbar und stellten damit einen MF dar. Am häufigsten standen Antiepileptika (v. a. Valproinsäure und Levetiracetam) und Antidiabetika (v. a. Insulin) in Verbindung mit einer vermeidbaren, arzneimittelbedingten stationären Aufnahme.

Schlussfolgerung: Diese Studie bestätigt, dass arzneimittelbedingte stationäre Aufnahmen ein bedeutendes Problem bei Kindern und Jugendlichen ist, knapp $50 \%$ dieser Aufnahmen sind vermeidbar. Ein gesteigertes Bewusstsein für pädiatrische Besonderheiten während der Arzneimitteltherapie und die Bereitstellung von standardisierten Informationen und Leitlinien könnten dazu beitragen, das Risiko arzneimittelbedingter stationärer Aufnahmen in der Pädiatrie zu reduzieren.

\footnotetext{
Literatur

1. Kimland et al (2012) Clin Pharmacol Ther 91:796-801

2. Wong et al (2004) Drug Saf 27:661-670

3. Smyth et al (2012) PLoS ONE 7:e24061

4. Rashed et al (2012) Eur J Clin Pharmacol 68:801-810

5. Gallagher et al (2012) PLoS ONE 7:e50127
}

\section{DGKJ-FV 16}

\section{Zusammenspiel zwischen sozialen Faktoren, Verhalten und Gesundheit im Kindes- und Jugendalter: Ergebnisse der Kohortenstudie LIFE Child}

Tanja Poulain', Mandy Vogel', Anne Jurkutat ${ }^{1}$, Andreas Hiemisch' ${ }^{2}$, Anja Hilbert ${ }^{3}$, Jon Genuneit ${ }^{4}$, Antje Körner ${ }^{5}$, Wieland Kiess ${ }^{6}$

${ }^{1}$ Universität Leipzig, LIFE Child, Kinder- und Jugendmedizin, Pädiatrisches Forschungszentrum, Leipzig, Deutschland; 'Universität Leipzig, LIFE Child, Kinder- und Jugendmedizin, Pädiatrisches Forschungszentrum, Leipzig; 'Universität Leipzig, LIFE Child, Leipzig; ${ }^{2}$ Universität Leipzig, Kinder- und Jugendmedizin, Pädiatrisches Forschungszentrum, Leipzig, Deutschland; ${ }^{3}$ Universität Leipzig, Medizinische Psychologie und Medizinische Soziologie, IFB AdipositasErkrankungen, Leipzig, Deutschland; ${ }^{4}$ Universität Leipzig, Pädiatrische Epidemiologie, Leipzig; ${ }^{5}$ Universität Leipzig, LIFE Child, Kinder- und Jugendmedizin, Pädiatrisches Forschungszentrum, IFB AdipositasErkrankungen, Leipzig; ${ }^{6}$ Universität Leipzig, LIFE Child, Kinderund Jugendmedizin, Pädiatrisches Forschungszentrum, Leipzig

Fragestellung: Die LIFE Child Studie ist eine Längsschnittstudie, die seit 2011 in Leipzig durchgeführt wird und der Frage nachgeht, wie sich (gesunde) Kinder entwickeln und welche Faktoren diese Entwicklung beeinflussen. Ein Forschungsbereich beschäftigt sich mit dem Zusammenspiel zwischen sozialen Faktoren, Gesundheits- und Freizeitverhalten und Gesundheit/Wohlbefinden.

Material und Methode: Im Rahmen der Studie werden Schwangere, Kinder und Jugendliche sowie Eltern einmal jährlich untersucht. Bisher haben mehr als 4500 Kinder und Jugendliche (davon 3300 zu mehreren Zeitpunkten) sowie 1000 Schwangere (davon 600 in der 24. und 36. Schwangerschaftswoche) an der Studie teilgenommen. Das Programm umfasst zahlreiche körperliche Untersuchungen, Tests, Befragungen zu Lebensumfeld und Freizeitgestaltung und die Erfassung biologischer Proben.

Ergebnisse: Die Ergebnisse bisheriger Untersuchungen zeigen, dass Kinder aus niedrigeren Sozialschichten einen signifikant höheren BMI haben, länger vor dem Fernseher sitzen, weniger Sport treiben, häufiger rauchen und ein geringeres Wohlbefinden angeben als Kinder aus höheren Sozialschichten. Unabhängig vom Sozialstatus konnte sportliche Betätigung mit einem höherem Wohlbefinden in Zusammenhang gebracht werden. Im Gegensatz dazu ging ein hoher Mediengebrauch mit schlechteren Schulnoten, mehr Schlafproblemen und mehr Verhaltensauffälligkeiten einher. Das elterliche Verhalten, vor allem der Gebrauch elektronischer Geräte und die Häufigkeit von Eltern-Kind-Interaktionen, konnte ebenfalls mit Verhaltensauffälligkeiten im Kindesalter assoziiert werden.

Schlussfolgerung: Das vielfältige Untersuchungsprogramm, das längsschnittliche Design und die tiefgreifende Phänotypisierung der Studie ermöglichen wichtige Einblicke in das Wechselspiel zwischen sozialen Faktoren, Gesundheitsverhalten und Gesundheit/Wohlbefinden. Sowohl der soziale Hintergrund als auch bestimmte Verhaltensweisen und die zwischenmenschliche Interaktion spielen eine Rolle für das Wohlbefinden und die Gesundheit von Kindern.

\section{Literatur}

1. Poulain T, Vogel M, Sobek C, Hilbert A, Körner A, Kiess W (2019) Associations Between Socio-Economic Status and. :

2. Child Health: Findings of a Large German Cohort Study. International Journal of Environmental Research and Public Health.

3. Poulain $T$, Voge 


\section{DGKJ-FV 17}

Depression ist häufiger bei pädiatrischen Patienten mit Typ-1Diabetes und Zöliakie: Deskription und Vergleich basierend auf einem großen multizentrischen Diabetesregister

Sascha R. Tittel', Désirée Dunstheimer', Dörte Hilgard', Burkhild Knauth4', Elke Fröhlich-Reiterer ${ }^{5}$, Angela Galler ${ }^{6}$, Michael Wurm ${ }^{7}$, Reinhard W. Holl'

'Universität Ulm, Institut für Epidemiologie und medizinische Biometrie, ZIBMT, Ulm, Deutschland; ${ }^{2}$ Universitätsklinikum Augsburg, Klinik für Kinder und Jugendliche, Augsburg, Deutschland; ${ }^{3}$ Kinder- und Jugendarztpraxis Dr. med. Dörte Hilgard, Kinder- und Jugendmedizin, Witten; ${ }^{4} \mathrm{CJD}$ Berchtesgaden, Diabeteszentrum, Berchtesgaden; ${ }^{5}$ Medizinische Universität Graz, Abteilung für Allgemeine Pädiatrie, Graz; ${ }^{6}$ Charité Universitätsmedizin Berlin, Pädiatrische Endokrinologie und Diabetologie, Berlin; ${ }^{7}$ Krankenhaus Barmherzige Brüder Regensburg, Klinik und Poliklinik für Kinder- und Jugendmedizin - Lehrstuhl der Universität Regensburg, Regensburg

Hintergrund: Zöliakie und Depression sind häufige Begleiterkrankungen bei Typ-1-Diabetes (T1D). Allerdings ist der Zusammenhang zwischen Zöliakie und Depression bei T1D bisher noch unklar.

Fragestellung: Anhand pädiatrischer T1D-Patienten soll untersucht werden, ob Depression bei T1D mit Zöliakie häufiger auftritt, verglichen mit T1D ohne Zöliakie.

Material und Methoden: Eingeschlossen wurden 78.799 T1D-Patienten zwischen 6 und 20 Jahren aus der Diabetes-Patienten-Verlaufsdokumentation (DPV). Gruppenvergleiche wurden für multiples Testen mit Bonferroni-Korrektur adjustiert. Hypoglykämie-Raten wurden mit negativbinomialen Regressionsmodellen berechnet. Logistische Regressionsmodelle für den Einfluss von Zöliakie auf Depression wurden nach Geschlecht, Alter, Diabetesdauer, Migrationshintergrund adjustiert. Die Einflüsse von Zöliakie und Depression auf den HbAlc und den BMI-SDS (Referenz: KiGGS) wurden mittels linearer Regression, adjustiert nach Alter, Geschlecht, Diabetesdauer und Migrationshintergrund, untersucht.

Ergebnisse: Von 78.799 eingeschlossenen T1D-Patienten wurde bei 12.027 zusätzlich Zöliakie (Z), bei 1324 Depression (D), und bei 361 Zöliakie und Depression $(\mathrm{D}+\mathrm{Z})$ diagnostiziert. Nur T1D lag bei 65.087 Patienten vor (T1DO). Dabei waren von Depression mit und ohne Zöliakie mehr Mädchen als Jungen betroffen (D+Z: 59,6\% weiblich, D: 56,5\% weiblich, Z: 49,9 \% weiblich). Patienten mit Zöliakie haben ein um 37 \% erhöhtes Risiko an Depression zu erkranken als Patienten ohne Zöliakie (OR: 1,37, $95 \%$-Konfidenzintervall: [1,21-1,54]).

Die Rate an schweren Hypoglykämien in Gruppe T1DO lag bei 0,14/Patientenjahr (PJ), in Gruppe Z bei $0,13 / \mathrm{PJ}$, in $\mathrm{D}$ bei $0,17 / \mathrm{PJ}$ und in $\mathrm{D}+\mathrm{Z}$ bei $0,24 / P J$. Einen signifikanten Unterschied gab es nur zwischen $Z$ und $\mathrm{D}+\mathrm{Z}(p=0,04)$.

Patienten in den Gruppen D, D+Z haben einen signifikant höheren HbAlc (beide 9,0\%) als Patienten in den Gruppen $\mathrm{Z}$ und T1DO (8,2\%, bzw. $8,3 \%$ ). Patienten aus Gruppe D hatten einen signifikant höheren BMI-SDS $(0,54)$ als Zöliakie-Patienten $(0,44)$ oder Patienten aus Gruppe G $(0,46)$. Mädchen mit Depression hatten einen höheren BMI-SDS als Mädchen mit Zöliakie; dies traf bei den Jungen nicht zu.

Schlussfolgerung: Kinder und Jugendliche mit T1D und Zöliakie erkranken im Vergleich zu Patienten ohne Zöliakie signifikant häufiger an einer Depression. Patienten mit Zöliakie hatten insgesamt einen niedrigeren $\mathrm{HbA1c}$, bei Mädchen dieser Gruppe war der BMI-SDS geringer. Hypoglykämien treten bei Patienten mit Zöliakie und Depression häufiger auf und der HbAlc liegt höher im Vergleich zu Patienten mit Zöliakie ohne Depression. Die Daten unterstreichen die Notwendigkeit des ZöliakieScreenings bei pädiatrischen Patienten mit T1D. Zudem sollte in der diabetologischen Betreuung besonders bei Kindern und Jugendlichen mit T1D und Zöliakie auf depressive Symptome geachtet werden.

\section{DGKJ-FV 18}

KUNO-Kids Gesundheitsstudie: Validierung des

Befundes „Hodenhochstand“ im Rahmen der U2Früherkennungsuntersuchung in der Kinderurologie

Martin Promm ', Annika Dittrich', Susanne Brandstetter ${ }^{3}$,Michael Kabesch', Michael Melter ${ }^{3}$, Birgit Seelbach-Göbel', Christian Apfelbacher ${ }^{5}$, Wolfgang H. Rösch ${ }^{6}$

${ }^{1}$ Kinder Uniklinik Ostbayern (KUNO), Universität Regensburg, Klinik St. Hedwig, Kinderurologie, Klinik für Kinderurologie in Kooperation mit der Universität Regensburg, Klinik St. Hedwig, Regensburg, Deutschland; ${ }^{2}$ Klinik St. Hedwig, Regensburg, Klinik für Kinderurologie in Kooperation mit der Universität Regensburg, Klinik für Kinderurologie in Kooperation mit der Universität Regensburg, Klinik St. Hedwig, Regensburg; ${ }^{3}$ Universität Regensburg (KUNO-Kliniken), Klinik St. Hedwig, Regensburg, Klinik und Poliklinik für Kinder- und Jugendmedizin, Klinik und Poliklinik für Kinder- und Jugendmedizin der Universität Regensburg (KUNO-Kliniken), Regensburg; ${ }^{4}$ Klinik für Gynäkologie und Geburtshilfe, Klinik St. Hedwig, Krankenhaus Barmherzige Brüder, Lehrstuhl für Geburtshilfe der Universität Regensburg, Regensburg, Klinik für Gynäkologie und Geburtshilfe, Klinik St. Hedwig, Krankenhaus Barmherzige Brüder, Lehrstuhl für Geburtshilfe der Universität Regensburg, Regensburg, Klinik für Gynäkologie und Geburtshilfe, Klinik St. Hedwig, Krankenhaus Barmherzige Brüder, Lehrstuhl für Geburtshilfe der Universität Regensburg, Regensburg; ${ }^{5}$ Institut für Epidemiologie und Präventivmedizin, Medizinische Soziologie, Universität Regensburg, Regensburg, Institut für Epidemiologie und Präventivmedizin, Medizinische Soziologie, Universität Regensburg, Regensburg, Institut für Epidemiologie und Präventivmedizin, Medizinische Soziologie, Universität Regensburg, Regensburg; ${ }^{6}$ Klinik für Kinderurologie in Kooperation mit der Universität Regensburg, Klinik St. Hedwig, Regensburg, Klinik für Kinderurologie in Kooperation mit der Universität Regensburg, Klinik für Kinderurologie in Kooperation mit der Universität Regensburg, Klinik St. Hedwig, Regensburg

Fragestellung: Der Hodenhochstand gehört mit einer Prävalenz von etwa 3-5 \% zu den häufigsten Fehlbildungen bei Jungen. Die Hodenlagekontrolle ist ein fester Bestandteil der U2-Früherkennungsuntersuchung, dadurch soll eine rechtzeitige Therapieeinleitung gewährleistet sein, um Folgeschäden zu verhindern. Inwieweit sich die Untersuchungsergebnisse der U2 in einer kinderurologischen Diagnostik bestätigen lassen, ist nicht bekannt. In dieser prospektiven Studie wurden Jungen mit dem auffälligen Untersuchungsbefund eines Hodenhochstands in der Kinderurologie vorgestellt.

Material und Methode: Im Zeitraum von Juni 2015 bis Juni 2018 wurden alle Jungen, die an der KUNO-Kids Gesundheitsstudie teilnahmen und für die Daten aus der U2 vorliegen, in diese Untersuchung aufgenommen. Die Knaben, die im Rahmen der U2 hinsichtlich der Hodenlage auffällig waren, wurden innerhalb von einem Tag in der Kinderurologie zur erneuten klinischen Untersuchung inkl. Ultraschall vorgestellt. Männliche Neugeborene, die eine mit Hodenhochstand assoziierte Diagnose sowie Pendelhoden hatten, wurden ausgeschlossen. Alle Daten wurden in einem elektronischen Dateneingabesystem erfasst. Die Auswertung erfolgte deskriptiv.

Ergebnisse: Von insgesamt 861 Jungen wurde bei 50 (5,8 \%) in der U2 ein Hodenhochstand festgestellt. 30 dieser Kinder konnten in der Kinderurologie erneut untersucht werden. Dabei konnte bei 13 (43,3\%) Patienten die Diagnose verifiziert werden, bei 17 (56,7\%) Jungen zeigte sich eine korrekte Lage beider Hoden im Skrotum. Eine zusätzlich durchgeführte Ultraschalluntersuchung der Hoden bestätigte in allen Fällen die jeweilige Diagnose.

Diskussion: Bei 57 \% der Kinder, die mit einem Hodenhochstand vorgestellt wurden, konnte die Diagnose in der Kinderurologie nicht verifiziert werden. Auch eine Studie aus den USA (Snodgrass et al. Pediatrics 2011) hatte gezeigt, dass bei $43 \%$ der Kinder, die mit der Verdachtsdiagnose „Hodenhochstand“ zur spezialisierten Diagnostik weitergeleitet wurden, kein Hodenhochstand vorlag. Der hohe Anteil falsch positiver Befunde in der U2 mag der ärztlichen Haltung geschuldet sein, im Zweifelsfall keine 
Diagnose übersehen zu wollen. Trotzdem sind falsch positive Befunde mit weiteren Untersuchungen und evtl. Verunsicherung der Eltern verbunden. Schlussfolgerung: Im Rahmen der U2 wurde der Befund eines Hodenhochstands in über $50 \%$ der Fälle falsch positiv gestellt. Weitere Untersuchungen sind notwendig, um mögliche Einflussfaktoren auf die Diagnosestellung zu eruieren und die Qualität zu verbessern.

\section{Literatur \\ 1. Snodgrass W, Bush N, Holzer M, Zhang S (2011) Current referral patterns and means to improve accuracy in diagnosis of undescended testis. Pediatrics 127(2):e382-8. https://doi.org/10.1542/peds.2010-1719 \\ 2. Brandstetter S, Toncheva AA, Niggel J, Wolff C, Gra}

\section{DGKJ-FV 19}

\section{Stationäre Versorgung des Morbus Wilson im Kindesalter}

\section{Steffen Wahler', Elena Wahler ${ }^{2}$, Alfred Mueller ${ }^{3}$}

'Praxis am Lerchenberg, Diabetologie, Hamburg, Deutschland; ${ }^{2}$ St. Bernward $\mathrm{GmbH}$, Epidemiologie, Hamburg; ${ }^{3}$ Analytic Services $\mathrm{GmbH}$, Demographie, München

Einführung: Morbus Wilson (MW) ist eine autosomal-rezessiv vererbte Erkrankung, bei der durch eine oder mehrere Genmutationen in der Leber die Kupferausscheidung über die Galle vermindert ist. Folge ist eine Ansammlung von Kupfer in Leber, Zentralnervensystem und anderen Organen. Es resultieren vielgestaltige Symptomen. Nach deutschen Registerdaten leiden $49 \%$ der Betroffenen an Leberschäden, $26 \%$ an neurologischen Defiziten, nur $12 \%$ an einer Kombination beider. In $13 \%$ ist der Verlauf asymptomatisch. In seltenen Fällen verläuft die Lebererkrankung kurzfristig fatal. Die genaue Inzidenz ist nicht bekannt und wird zwischen 1:30.000 und 1:300.000 angegeben.

Hier soll untersucht werden, wie frequent Kinder und Jugendliche mit MW stationär behandelt werden und welche der Symptome dabei im Vordergrund stehen. Ebenso sollen Alters- und Geschlechtsstruktur mit Registerdaten verglichen werden.

Methoden: Daten der stationären Versorgung des statistischen Bundesamtes 2005-2016 und die Qualitätsberichte für das Jahr 2015 wurden auf MW-Kodierungen untersucht und ausgewertet. Verwendet wurden Microsoft-Excel und Microsoft-Access (Version 2016).

Ergebnisse: Während sich im deutschlandweiten Register 59 \% weibliche Patienten finden, machen diese nur $45 \%$ der stationären Fälle aus. 14,6 \% der Patienten sind Kinder und Jugendliche bis 18, Jahre über die Hälfte weiblich. Frauen sind im Mittel kürzer stationär: Verweildauer 6,2 Tage versus 8,5 Tage. Patienten werden zu $45 \%$ in rein neurologischen $\mathrm{Ab}$ teilungen behandelt, $20 \%$ Pädiatrie und $29 \%$ Gastroenterologie. $3 \%$ der Fälle in Transplantationseinheiten. Da die Aufenthalte in der Neurologie länger dauern, sind weit über Hälfte der Verweiltage dort. Die Anzahl der Fälle liegt seit 2005 betrachtet konstant um 600. Der Altersdurchschnitt be Hauptdiagnose MW liegt bei 30,1 Jahren (Median 28,0) ohne Geschlechtsunterschied ( $\mathrm{m} 29,9$; w 30,3). Auffällig ist eine Lücke bei Patienten, die zwischen dem 20. und 25. Lebensjahr stationär behandelt werden.

Zusammenfassung: Im stationären Bereich finden sich unterschiedliche Verteilungen des MW als in den Registerdaten. Es sind mehr neurologische Krankheitsbilder die im Krankenhaus behandelt werden. Knapp $15 \%$ der stationären Wilson Fälle sind Kinder. Eine Lücke im Alter ab 20 Jahre spricht für eine suboptimale Transition.

\section{Innovative Diagnostik}

\section{DGKJ-FV 20}

\section{(Trio-)exome sequencing and beyond in routine diagnostics}

Lucia Laugwitz', Rebecca Buchert², Stefanie Beck-Wödl', Mona Grimmel', Marc Sturm², Darja Gauck², Ute Grasshoff2, Olaf Rieß², Tobias Haack ${ }^{2}$

'Universitätsklinikum Tübingen, Universitätsklinik für Kinder- und Jugendmedizin, Humangenetik, Tübingen, Germany; ${ }^{2}$ Universität Tübingen, Humangenetik, Tübingen, Germany

Background: (Trio-)exome sequencing has proven a powerful tool to establish new disease genes and molecular diagnoses in Rare diseases. However, a significant portion of affected individuals remains undiagnosed arguing for the implementation of technical (bioinformatics) standards for NGS data analysis, application of full genome sequencing as a first line diagnostic approach and pro-active sharing of diagnostic datasets with the scientific community. Using a cohort of 279 index cases with clinically suspected neurometabolic disorders (NMDs) as an example, we highlight factors influencing the success rate of different sequencing approaches, implicit issues such as management of incidental findings and guidance of downstream investigations in unsolved cases.

Methods: Exome and full genome sequencing was perfomed using a SureSelect Human All Exon Kit V6/7 (Agilent technologies) or an Illumina PCR-free protocol, respectively. Sequencing was performed on an Illumina HiSeq2500/NovaSeq6000 system as paired-end reads. Generated sequences were analyzed using the megSAP pipeline (https://github.com/imgag/ megSAP) and a standardized filtering algorithm for variant prioritization including a search for copy numbers and mtDNA variants.

Results: In $40 \%$ of the cases samples from the parents of affected sibling were available for first line trio-/multi-sample analyses. In $43 \%$ of the cohort, we identified likely pathogenic or pathogenic variants (class 4 or 5) in established disease genes associated with neuropediatric phenotypes. In $32 \%$ of these cases a targeted therapy was available; in 33\% the definitive molecular diagnosis implicated a modification of clinical management. In $26 \%$ of the cases, we identified variants of unknown significance and functional follow-up studies (e.g. metabolic, transcriptome analyses) and/or neuroradiological reevaluations were initiated. We prioritized 10 new candidate genes currently pending functional studies and/or identification of additional families. In $27 \%$ of the cases the molecular cause remained unclear. Besides sharing of the generated phenotypic and genetic data within European research networks (e.g. SolveRD) full genome and/ or transcriptome sequencing was initiated.

Conclusions: Definition of advanced standards for exome data analysis and sharing is crucial to maximize the diagnostic yield and realize personalized treatment concepts. While full genome sequencing is already in place as a first line diagnostic tool for some indications, joint efforts of families, clinicians and management is needed to translate complementary multi-omics approaches currently established in a research context into clinical routine.

\section{DGKJ-FV 21}

\section{Nachweis genomischer Mutationen in FAT1 und GLI1 bei VACTERL-} Assoziation

Jessica Ritter ${ }^{1}$, Kristina Lisec ${ }^{1}$, Dietrich Schweinitz', Roland Kappler ${ }^{2}$, Jochen Hubertus ${ }^{1}$

'Dr. von Haunersches Kinderspital der Universität München, Kinderchirurgie, München, Deutschland; ${ }^{2}$ Dr. von Haunersches Kinderspital der Universität München, Kinderchirurgisches Forschungslabor, München, Deutschland

Fragestellung: Die VACTERL-Assoziation stellt einen Fehlbildungskomplex dar, der mindestens drei Fehlbildungen der folgenden Organsysteme aufweist: Vertebra, Anorektum, Herz und Gefäße, Trachea mit Ösophagus, Urogenitaltrakt, sowie Extremitäten. „Assoziation“ beschreibt dabei den Zusammenhang des gemeinsamen Auftretens der Fehlbildungen bei niedriger Einzelinzidenz. Die genaue Ursache der VACTERL-Assoziation ist bisher ungeklärt, jedoch wird mitunter von einem multifaktoriellen ge- 
netischen Zusammenhang ausgegangen. Die vorliegende Studie stellt ein Mutations-Screening bei Kindern mit VACTERL-Assoziation dar. Methoden: Einundzwanzig Patienten im Alter zwischen einem Monat und dreißig Jahren mit mindestens drei betroffenen Organsystemen bei VACTERL-Assoziation wurden mit Hilfe einer Whole-Exome-Sequenzierung (WES) untersucht. Ausgesuchte Genveränderungen wurden anschließend mittels Sanger-Sequenzierung verifiziert.

Ergebnisse: In der WES zeigten sich in unserem Patientenkollektiv insgesamt 3997 Veränderungen der Nukleotidsequenz, die einen Einfluss auf das Protein haben. Interessanterweise war bei vier (19\%) Patienten FAT1 und bei zwei (9,5\%) Patienten GLI1 verändert, Gene die in den Wnt- bzw. den Hedgehog-Signalwegen involviert sind. Die Sanger-Sequenzierung verifizierte diese Veränderungen. Einer der insgesamt sechs Mutationsträger wies gleichzeitig eine FAT1 und GLI1 Veränderung auf. Zudem zeigten alle sechs FAT1 bzw. GLI1 Mutationsträger als gemeinsame phänotypische Ausprägung eine Ösophagusatresie Typ IIIb nach Vogt.

Diskussion: FAT1 und GLI1 sind an der Embryonalentwicklung beteiligt und können daher potenziell Fehlbildungen verursachen, jedoch kann die VACTERL-Assoziation nicht vollständig durch diese beiden Gene erklärt werden. In Zusammenschau mit weiteren, aus der Literatur bekannten, genetischen Veränderungen unterstützen FAT1 und GLI1, mit dem Auftreten von Veränderungen bei vier bzw. zwei von einundzwanzig untersuchten VACTERL-Patienten (19\% bzw. 9,5\%), die These einer multigenetischen Assoziation dieses Fehlbildungskomplexes.

Schlussfolgerung: Zusammengefasst stellen FAT1 und GLI1 zwei Kandidatengene für die VACTERL-Assoziation dar.

\section{DGKJ-FV 22}

\section{Hämatologische Veränderungen bei sehr unreifen Frühgeborenen mit schwerer intrauteriner Wachstumsretardierung}

Tobias Mühlbacher, Nora Johanna Reibel, Christoph Bührer, Christof Dame Charité - Universitätsmedizin Berlin, Neonatologie, Berlin, Deutschland

Fragestellung: Untersuchung der Assoziation zwischen Veränderungen im Blutbild (Thrombozyto-/Leukopenie) und einem erhöhten Infektionsund Blutungsrisiko sowie der Mortalitätsrate bei sehr unreifen Frühgeborenen (FG) mit schwerer intrauteriner Wachstumsrestriktion (IUGR). Methode: In diese retrospektive Fall-Kontroll-Studie (1:2 Allokation) wurden alle FG mit einem Gestationsalter (GA) $<28+0$ Schwangerschaftswochen (SSW) und schwerer IUGR (Geburtsgewicht $\leq 3$. Perzentile) einbezogen, die zwischen 2008 und 2017 geboren wurden. Als Kontrollen wurden FG mit Geburtsgewicht $>10$. Perzentile ausgewählt, die nach Geschlecht, GA und Geburtsjahr möglichst der Fallgruppe entsprachen. Blutbild, Infektionsdiagnostik, antimikrobielle Therapie, Transfusion von Blutprodukten innerhalb der ersten Lebenswoche, sowie neonatale Komplikationen (Infektionen; intraventrikuläre (IVH) und pulmonale Hämorrhagie $(\mathrm{PH})$ ) und die Mortalitätsrate wurden erhoben.

Ergebnisse: 55 FG wurden in die Fallgruppe, 110 FG in die Kontrollgruppe eingeschlossen (Geburtsgewicht $430 \mathrm{~g}$ (270-570 g) vs. $798 \mathrm{~g}$ (514$1245 \mathrm{~g}$ )), das mediane GA betrug $25+3$ SSW in der Fall- und $25+4$ SSW in der Kontrollgruppe (n.s.). In der Fallgruppe war die Erythroblastenzahl erhöht (251 vs. $25 / 100$ Leukozyten, $p<0,001)$, die Anzahl der Leukozyten (4,9 vs. $9,4 / \mathrm{nl} ; p<0,001)$ und Thrombozyten (115 vs. $220 / \mathrm{nl} ; p<0,001)$ erniedrigt. Eine Chorioamnionitis (IL6 $>100 \mathrm{ng} / \mathrm{l}$ oder $\mathrm{CrP}>10 \mathrm{mg} / \mathrm{l}$ ) trat häufiger in der Kontrollgruppe auf $(21,8 \%$ vs. $38,2 \%, p<0,05)$, die Fallgruppe wies dagegen eine höhere Prävalenz erhöhter systemischer Inflammationsparameter (cut-off Werte s. o.) als Zeichen von early ( $<72 \mathrm{~h})$ oder late onset $(>72 \mathrm{~h}$ ) Infektionen auf. Es bestand kein Unterschied hinsichtlich von Blutungskomplikationen (IVH alle Stadien, $\mathrm{PH}$ ), jedoch war die Mortalitätsrate mit 36,4 \% vs. 7,3\% $(p<0,001)$ bei IUGR erhöht, in der multiplen Regression auch unabhängig von GA und Geburtsgewicht $(p<0,05)$

Innerhalb der Fallgruppe war die Mortalität mit einer höheren Rate an IVH und $\mathrm{PH}$ assoziiert ( $40 \%$ vs. $5,7 \% ; p<0,01$ ). Bei den verstorbenen IUGR-Patienten fiel ein erhöhtes Transfusionsvolumen von Thrombozy- tenkonzentrat ( 42 vs. $17 \mathrm{ml} / \mathrm{kg}, p<0,01$ ), jedoch nicht von Gefrierplasma (33 vs. $22 \mathrm{ml} / \mathrm{kg}$; n.s.) auf.

Diskussion: Unsere Untersuchung bestätigt die Assoziation der early onset Thrombozyto- und Leukopenie mit einer gesteigerten Erythropoiese bei schwerer IUGR. Die höhere Inzidenz von Infektionen bei Leukopenie wirft die Frage auf, ob und ab welcher Leukozytenzahl eine präventive antimikrobielle Therapie begründet ist. Es bedarf einer kritischen Prüfung, inwiefern der erhöhte Transfusionsbedarf in der Subgruppe verstorbener FG mit IUGR mit höherer Inzidenz von Blutungskomplikationen assoziiert ist.

Schlussfolgerung: Extreme Wachstumsretardierung ist, insbesondere bei Vorliegen von IVH und PH, mit einer höheren Mortalität assoziiert. Strategien zum Management bei Leukopenie und Thrombozytopenie bedürfen einer besonderen Aufmerksamkeit.

\section{DGKJ-FV 23 \\ S100A8/A9 ist der erste prädiktive Biomarker für das Sepsisrisiko eines Frühgeborenen}

Sabine Pirr', Louise Dauter ${ }^{2}$, Bettina Bohnhorst' ${ }^{2}$, Corinna Peter ${ }^{2}$, Martin Stanulla', Johannes Roth ${ }^{3}$, Gesine Hansen'2, Thomas Vogl', Dorothee Viemann ${ }^{2}$

'Medizinische Hochschule Hannover, Klinik für Pädiatrische Pneumologie, Allergologie und Neonatologie, Hannover, Deutschland; ${ }^{2}$ Medizinische Hochschule Hannover, Pädiatrische Pneumologie, Allergologie und Neonatologie, Hannover, Deutschland; ${ }^{3}$ Westfälische Wilhelms-Universität Münster, Institut für Immunologie, Münster, Deutschland

Fragestellung: Die Sepsis ist eine der Haupttodesursachen in der Neonatalperiode. Vor allem Frühgeborene (FG) mit einem Gestationsalter (GA) $<32$ Schwangerschaftswochen (SSW) haben ein hohes Risiko, an einer lateonset Sepsis (LOS) zu erkranken. Aus Angst vor Infektionen und aufgrund der eingeschränkten Sensitivität und Spezifität konventioneller Biomarker werden FG oft präemptiv antibiotisch behandelt. Ein prädiktiver Biomarker für das Sepsisrisiko wäre hilfreich, um unnötige Antibiotikagaben zu verhindern.

Die Kaiserschnittentbindung wurde jüngst als erster Faktor identifiziert, der bei ansonsten gesunden FG zum Zeitpunkt der Geburt ein erhöhtes Sepsisrisiko anzeigt. Wir konnten kürzlich eine starke Korrelation zwischen niedrigen Spiegeln des immunregulatorischen Alarmins S100A8/ A9 und einer erhöhten Sepsisinzidenz bei Frühgeborenen aufdecken. Es stellte sich die Frage, ob dieser Assoziation eine Abhängigkeit der S100A8/ A9 Spiegel vom Geburtsmodus zugrunde liegt oder ob S100A8/A9 einen unabhängigen prädiktiven Marker für die neonatale Sepsis darstellt.

Material und Methoden: In die Studie wurden 110 Frühgeborene mit einem GA <32 SSW ohne Hinweise auf infektiöse oder inflammatorische Erkrankungen zur Geburt eingeschlossen. Die Wahrscheinlichkeit des Auftretens einer LOS wurde in Abhängigkeit vom Geburtsmodus und dem S100A8/A9-Serumspiegel am ersten Lebenstag ermittelt.

Ergebnisse: Im Mittel waren die S100A8/A9-Serumspiegel nach Spontanentbindung signifikant höher als nach Geburt per Kaiserschnitt $(\mathrm{P}=0,0234)$. Das Risiko einer LOS war nach Kaiserschnittentbindung doppelt so hoch wie nach Spontangeburt (OR 2,25, 95 \% CI 0,48-10,6), bei jedoch niedriger Signifikanz für diese Assoziation $(\mathrm{P}=0,305)$. Dahingegen war eine hohe Freisetzung von S100A8/A9 bei Geburt mit Serumspiegeln oberhalb der 25 . Perzentile $(>417 \mathrm{ng} / \mathrm{ml})$ mit einem 26 -fach geringeren Risiko einer LOS assoziiert (OR 0,039, 95 \% CI 0,011-0,14, P <0,0001) verglichen mit einer geringen oder fehlenden Freisetzung der Alarmine mit Serumspiegeln $\leq 417 \mathrm{ng} / \mathrm{ml}$. Auch innerhalb der Gruppe der per Kaiserschnitt entbundenen Kindern waren hohe Alarmin Serumspiegel mit einem 16-fach geringeren LOS-Risiko assoziiert (OR 0,062, CI 0,018-0,212, $\mathrm{P}<0,0001)$. Bei Serumspiegeln $<270 \mathrm{ng} / \mathrm{ml}$ ( $15 \%$ der Frühgeborenen in unserer Kohorte) stieg das Sepsisrisiko auf das 103-fache an. Unterhalb dieses Grenzwertes zeigte sich ein positiver Vorhersagewert von $0,88 \mathrm{mit}$ einer Sensitivität von 0,7 , einer Spezifität von 0,98 und einem negativen prädiktiven Wert von 0,94 für das LOS-Risiko. 
Schlussfolgerung: S100A8/A9-Spiegel erweisen sich als erster reliabler und geburtsmodus-unabhängiger Biomarker sowohl zur Vorhersage eines deutlich erhöhten LOS-Risikos als auch zur Identifizierung FG, bei denen die Wahrscheinlichkeit für das Erleiden einer LOS gegen Null strebt.

\section{DGKJ-FV 24}

\section{TNF- $a$ ist essentieller Mediator der postnatalen Einwanderung von Leukozyten in Organe und Grenzflächen}

Sabine Pirr', Marie Sophie Bickes², Anna Sophie Heinemann², Beate Fehlhaber', Stephan Halle ${ }^{3}$, Lena Völlger ${ }^{2}$, Maike Willers'2, Richter Manuela ${ }^{4}$, Carolin Böhne ${ }^{2}$, Melanie Albrecht ${ }^{2}$, Melissa Langer ${ }^{5}$, Sandra Pfeifer ${ }^{5}$, Danny Jonigk ${ }^{6}$, Gertrud Vieten ${ }^{7}$, Benno Ure ${ }^{7}$, Constantin von Kaisenberg ${ }^{8}$, Reinhold Förster $^{3}$, Maren von Köckritz-Blickwede ${ }^{5}$, Gesine Hansen ${ }^{2}$, Dorothee Viemann ${ }^{2}$ 'Medizinische Hochschule Hannover, Klinik für Pädiatrische Pneumologie, Allergologie und Neonatologie, Hannover, Deutschland; ${ }^{2}$ Medizinische Hochschule Hannover, Pädiatrische Pneumologie, Allergologie und Neonatologie, Hannover; ${ }^{3}$ Medizinische Hochschule Hannover, Institut für Immunologie, Hannover; ${ }^{4}$ Kinderkrankenhaus Auf der Bult, Neonatologie, Hannover; ${ }^{5}$ Tierärztliche Hochschule Hannover, Institut für Physiologische Chemie, Hannover; ${ }^{6}$ Medizinische Hochschule Hannover, Pathologie, Hannover; ${ }^{7}$ Medizinische Hochschule Hannover, Kinderchirurgie, Hannover; ${ }^{8}$ Medizinische Hochschule Hannover, Klinik für Frauenheilkunde und Geburtshilfe, Hannover

Hintergrund: In der Neonatalzeit finden weitreichende immunologische Änderungen auf zellulärer und molekularer Ebene statt. Fetale gewebsständige Leukozyten werden vielerorts durch einwandernde hämatopoetische Zellen ersetzt. Klinisch geht diese Phase mit einer erhöhten Anfälligkeit für Infektionen und Sepsis einher. In der adulten Sepsis ist das Endothel zentral an der Eskalation der systemischen Entzündungsreaktion beteiligt. Welche Rolle das Endothel in der Pathogenese der neonatalen Sepsis oder für die postnatalen Zellaustauschvorgänge spielt ist weitgehend undefiniert.

Fragestellung: 1. Tragen Entzündungsantworten des Endothels kritisch zur Pathogenese der neonatalen Sepsis bei? 2. Stellt die Hemmung der Endothelaktivierung eine mögliche präventive oder therapeutische Strategie zur Behandlung septischer Erkrankungen bei Neugeborenen dar? 3 Wie ist das Endothel in die Prozesse der postnatalen Leukozytenwanderung involviert?

Material und Methoden: Es wurden Serumspiegel löslicher Endothelmarker in gesunden und septischen Neugeborenen und gesunden Erwachsenen bestimmt. Funktionell wurde die inflammatorische Antwort humaner neonataler Endothelzellen in Medium-Transfer-Experimenten untersucht Die Effekte einer TNF-Blockade direkt nach Geburt auf Leukozyten-Endothel-Interaktionen wurden in der neonatalen Maus studiert und zwar i) in Gesundheit und ii) im Rahmen einer Endotoxinämie und iii) einer Staphylococcus (S.) aureus-induzierten Sepsis.

Ergebnisse: Lösliche Endothelmarker im Serum gesunder Reif- und Frühgeborener waren im Vergleich zu Erwachsenen signifikant erhöht und stiegen im Rahmen einer Sepsis nicht weiter an. Die massive basale Endothelaktivierung ließ sich auf eine neonatal hohe Produktion von TNF- $\alpha$ zurückführen. Die pharmakologische Blockade von TNF- $\alpha$ bei Geburt begrenzte zwar nachfolgende systemische Entzündungsreaktionen, erhöhte jedoch das Versterben an einer S. aureus-induzierten Sepsis infolge gehemmter Leukozytenrekrutierung. Insbesondere aber wurden auch die physiologischen Leukozytenrekrutierungsprozesse in der gesunden neugeborenen Maus durch eine neonatale TNF- $\alpha$-Blockade gestört; veränderte Leukozytenprofile in Lunge und Darm ließen sich bis ins junge Erwachsenenalter nachweisen.

Schlussfolgerung: Unsere Studie identifiziert TNF- $\alpha$ erstmalig als Vermittler einer starken basalen Endothelaktivierung in der Neonatalperiode. Diese prädisponiert Neugeborene nicht zu septischen Krankheitsbildern, sondern ist essentiell für die postnatale Einwanderung von Leukozyten in Organe und Grenzflächen.

\section{DGKJ-FV 25}

\section{Analyse des Herzfrequenzverhaltens unter Belastung bei Kindern} und jungen Erwachsenen mit angeborenem Herzfehler

Stephanie Meier ${ }^{1}$, Fabian von Scheidt ${ }^{2}$, Christian Apitz ${ }^{2}$

'Landshut, Deutschland; ${ }^{2}$ Universitätsklinikum Ulm, Klinik für Kinder und Jugendmedizin, Kardiologie, Augsburg

Hintergrund: Kinder und Jugendliche mit angeborenem Herzfehler (AHF) weisen häufig eine verminderte körperliche Belastbarkeit auf. Bei Erwachsenen mit AHF konnte gezeigt werden, dass die Herzfrequenzantwort unter Belastung zur Leistungseinschränkung beiträgt. Systematische Untersuchungen bei Kindern und Adoleszenten zu diesem Thema existieren bisher nicht.

Ziel dieser Untersuchung war es daher, die Herzfrequenzantwort unter Belastung und deren Einfluss auf die Belastungslimitation bei Kindern und Jugendlichen mit AHF zu untersuchen und mit einer herzgesunden Kontrollgruppe zu vergleichen.

Methoden: 163 Kinder und Adoleszente (103 mit AHF (medianes Alter 15 Jahre) und 60 altersanaloge herzgesunde Probanden) wurden auf dem Laufband spiroergometrisch untersucht. Neben der maximalen Sauerstoffaufnahme wurde die Herzfrequenzreserve (Differenz aus maximal erreichter Herzfrequenz und Ruheherzfrequenz) und der Herzfrequenzabfall während der Erholungsphase nach Belastungsende (Herzfrequenzerholung) gemessen.

Der chronotrope Index wurde definiert als prozentualer Anteil der altersentsprechend vorhergesagten maximalen Herzfrequenzreserve. Gemäß Literaturdaten erfolgte die Definition einer chronotropen Inkompetenz (CI) als chronotroper Index $<0,8$.

Ergebnisse: Maximale Herzfrequenz, Herzfrequenzreserve, und der chronotrope Index lagen bei AHF-Patienten niedriger als in der Kontrollgruppe. Auch die Herzfrequenzerholung 1 und 2 min nach Belastungsende war bei AHF-Patienten beeinträchtigt und korrelierte mit der maximalen Sauerstoffaufnahme.

Ein chronotroper Index $<0,8$ zeigte sich relativ häufig, sogar in der herzgesunden Kontrollgruppe, was nahelegte, dass die Schwelle von 0,8 für die Identifizierung der CI bei Laufbandspiroergometrien im Kindesalter inadäquat hoch erscheint. Nach Normalisierung bezogen auf die 2,5te Perzentile des chronotropen Index der herzgesunden Kontrollgruppe wurde ein revidierter Schwellenwert von 0,69 berechnet.

Schlussfolgerung: Aufgrund unserer Ergebnisse scheint die Herzfrequenzantwort unter Belastung ein physiologisch und klinisch bedeutender diagnostischer Parameter bei Kindern und Adoleszenten mit AHF zu sein. Jedoch müssen bei der Interpretation der Herzfrequenzantwort die altersspezifischen Besonderheiten und die speziellen Charakteristika der Methode der Belastungsuntersuchung berücksichtigt werden.

Die Ergebnisse unserer Analyse können dazu beitragen, zukünftige Studien zur CI bei Kindern und Jugendlichen unter Verwendung von Laufbandergometrie-Protokollen besser interpretieren zu können.

\section{DGKJ-FV 26}

\section{Manipulationen von Arzneiformen auf pädiatrischen Stationen eines deutschen Universitätsklinikums - ein unterschätztes Problem?}

Julia Zahn, André Hörning, Wolfgang Rascher, Regina Trollmann, Antje Neubert

Universitätsklinikum Erlangen, Kinder- und Jugendklinik, Erlangen, Deutschland

Hintergrund: Die Pharmakotherapie bei Kindern erfordert altersgerechte Arzneiformen in flexiblen Dosisstärken.[1,2] Aufgrund des Mangels an entsprechenden Medikamenten sind Manipulationen von Arzneimitteln, z.B. Teilen oder Mörsern von Tabletten, gängige Praxis in der Pädiatrie. Diese bergen jedoch Risiken, da sie Dosierungsgenauigkeit, Bioverfügbarkeit und Integrität der Arzneiform beeinflussen und somit zu Über- oder Unterdosierungen führen können.[2-4] Ziel der vorliegenden Studie war 
es, das Ausmaß und die Art der Manipulationen von oral verabreichten Arzneimitteln in einer deutschen Kinderklinik zu untersuchen.

Methode: Im Rahmen einer prospektiven Beobachtungsstudie wurden alle Manipulationen oral verabreichter Arzneimittel auf zwei Stationen einer deutschen Universitätskinderklinik identifiziert. An zufällig ausgewählten Tagen im Februar und März 2019 wurden die Stellprozesse der Arzneimittel, die vom Pflegepersonal durchgeführt wurden, systematisch erfasst und analysiert. Die Daten wurden deskriptiv ausgewertet und die Ursachen der Manipulationen ermittelt. Der Zulassungsstatus der Manipulation wurde laut Fachinformation beurteilt. Die Vermeidbarkeit der Manipulationen wurde anhand der Verfügbarkeit von alternativen Fertigarzneimitteln auf dem deutschen Markt untersucht.

Ergebnisse: Während des Beobachtungszeitraums wurden 528 Stellprozesse bei 71 Patienten beobachtet. In 38,4 \% dieser Prozesse wurden Arzneimittel manipuliert. 57,7\% der Patienten waren von mindestens einer Manipulation betroffen. Tabletten (64\%), Tabletten mit veränderter Wirkstofffreisetzung (15\%), Kapseln (7\%) und Infusionslösungen zur oralen Anwendung (7 \%) waren die am häufigsten manipulierten Darreichungsformen. Zudem wurden Vitamine (24,7 \%), Arzneimittel gegen säurebedingte Störungen (18,0 \%) und Antiepileptika (13,5\%) besonders häufig manipuliert. Der größte Anteil an Manipulationen wurde bei Kindern $(45,8 \%)$ und Säuglingen/Kleinkindern $(42,3 \%)$ beobachtet. Die Hauptursachen für die Durchführung einer Manipulation waren das Vorliegen des Arzneimittels in ungeeigneter Stärke bzw. ungeeigneter Darreichungsform. 51,7 \% der Manipulationen waren laut Fachinformation nicht zugelassen. In 38,8 \% gab es kein alternatives, kommerziell erhältliches Arzneimittel um eine nicht zugelassene Manipulation zu verhindern.

Diskussion und Schlussfolgerung: Die Ergebnisse der Studie zeigen, dass Manipulationen von Arzneimitteln auf Stationen einer deutschen Universitätskinderklinik gängige Praxis sind. Trotz regulatorischer Bestrebungen die Arzneimitteltherapie für Kinder zu verbessern, besteht noch immer ein Mangel an pädiatrischen Medikamenten. Weitere Untersuchungen sind erforderlich um die damit verbundenen Risiken abzuschätzen und die Vermeidbarkeit von Manipulationen bestimmen zu können.

\section{Literatur \\ 1. Schirm E, Tobi H, de Vries TW, Choonara I, De Jong van den Berg LT (2003) Lack of appropriate formulations of medicines for children in the community. Acta Paedia- tr 92(12):1486-1489 \\ 2. Richey RH, Craig JV, Shah UU, Nunn A}

\section{DGKJ-FV 27}

Eliciting dose is associated with tolerance development in peanut and cow's milk allergic children/Die Allergendosis bei Diagnosestellung von kindlicher Erdnuss- und Milchallergie steht im Zusammenhang mit natürlicher Toleranzentwicklung

Catharina Nitsche', C.D. Westerlaken-van Ginkel', B. J. Kollen², A. B. Sprikkelman'2, G. H. Koppelman'2, A. E.J. Dubois

'University of Oldenburg, Germany; University of Groningen, The Netherlands; University Medical Center Groningen, Department of Paediatric Pulmonology and Paediatric Allergology, GRIAC Research Institute, Groningen, The Netherlands, Groningen, Netherlands; ${ }^{2}$ University of Groningen, University Medical Center Groningen, Department of Paediatric Pulmonology and Paediatric Allergology, GRIAC Research Institute, Groningen, Netherlands

Background: Tolerance development rates differ between food allergies [1, 2]. Almost all previous studies have not used the gold standard method, the double-blind, placebo-controlled food challenge (DBPCFC), which may affect the reported prevalence rates. Little is known about the association of the eliciting dose (ED) obtained during the initial DBPCFC with later tolerance development.

Methods: This was a retrospective, tertiary care study of children who had a positive DBPCFC to either peanut, milk or egg, and at least one followup food challenge (open or DBPCFC) with the same food. The association between ED and negative (tolerant) follow-up food challenge outcome was analyzed by logistic regression, with adjustment for confounders. Suspected confounders were initial DBPCFC test characteristics, atopic comorbidities and serum specific IgE (sIgE) levels.

Results: In 47 peanut allergic children, tolerance developed in $27.7 \%$ (median follow-up duration of 43 months). In 80 milk (follow-up 23 months) and 55 egg (follow-up 37 months) allergic children, tolerance developed in 55.0 and $65.5 \%$. The ED obtained during the initial DBPCFC was significantly associated with tolerance development in peanut and milk allergy, but not in egg allergy.

Conclusion: Approximately 1 out of 4 children with DBPCFC confirmed peanut allergy developed tolerance, compared to more than half of the children with milk or egg allergy, respectively. Tolerance development in peanut and milk allergy is significantly associated with ED at initial DBPCFC.

\section{References}

1. Schoemaker AA, Sprikkelman AB, Grimshaw KE et al (2015) Incidence and natural history of challenge-proven cow's milk allergy in European children-EuroPrevall birth cohort. Allergy 70(8):963-972

2. Peters RL, Dharmage SC, Gurrin LC, et al. The natural

\section{DGKJ-FV 28}

\section{Smarty - Internetbasierte Kommunikation in der Versorgung chronisch kranker Kinder}

Ute Graepler-Mainka' , Christian Wolf ${ }^{2}$, Ekkehard Sturm?', Martin Holderried ${ }^{2}$, Ansgar Höper ${ }^{3}$, Christian Ernst ${ }^{4}$

'Universitätsklinik für Kinder- und Jugendmedizin Tübingen, Allgemeine Pädiatrie, Tübingen, Deutschland; ${ }^{2}$ Universitätsklinik Tübingen,

Zentralbereich Medizin-, Struktur-, Prozess- und Qualitätsmanagement, Tübingen, Deutschland; ${ }^{3}$ Universitätsklinik Tübingen, Zentralbereich Medizin, Tübingen, Deutschland; ${ }^{4}$ Universität Hohenheim - Lehrstuhl Ökonomik und Management sozialer Dienstleistungen, Institut für Health Care and Public Management, Stuttgart, Deutschland

Fragestellung: In der Tübinger Kinderklinik soll die Versorgung der Patienten mit Mukoviszidose und in der Nachsorge nach Lebertransplantation durch Einführung der Kommunikationsplattform Smarty (Social Medical Aplication Platform for children with Respitatory Dependency, Transplantation and Cystic Fibrosis) verbessert werden. Die Kommunikation zwischen Ärzten der Kinderklinik, Patienten, ihren Angehörigen und den niedergelassenen Kinder- und Hausärzten soll durch eine schnelle, datensichere und asynchrone Übermittlung von Informationen effektiver gestaltet werden.

Methoden: Es wurde eine Online-Plattform mit Ende-zu-Ende-Verschlüsselung eingeführt. Der Patient/die Eltern haben die Datenhoheit und entscheiden, wer in den virtuellen Raum eintreten darf. Über Smarty werden Berichte, Befunde, organisatorische und auch medizinische Nachrichten vom jeweiligen Teilnehmer online eingestellt. Die Erreichbarkeit der Teilnehmer und die Verfügbarkeit der Informationen sind durch eine asynchrone Kommunikation von jedem PC, Tablet oder Smartphone möglich. Durch die Verschlüsselungstechnik ist die Übermittlung sicher und frei von Verwechslungen, da die Datenübermittlung der patienteneigenen ID-Nummer zugeordnet ist.

Ergebnisse: Die Anwendung von Smarty wurde seit Januar 2018 zunehmend in den Alltag der Ambulanzen integriert. Es zeigte sich ein kontinuierlicher Anstieg der Nutzerzahlen besonders bei Patienten und Angehörigen. Zur Abschlussevaluation wurde ein standardisierter Fragebogen an alle Patienten/Patienteneltern (180) und Behandler (30) versendet, wodurch die intersektorale Kommunikation aus der Sicht der Teilnehmer erfasst werden sollte. Die Form der Kommunikation wird von $82 \%$ bzw $77 \%$ als gut bis sehr gut bewertet. Durch die online Kommunikation geben $86 \%$ der Patienten/Eltern und $93 \%$ der Behandler an, dass die Datensicherheit zugenommen habe. Im weiteren fühlen sich $81 \%$ der Patienten/Eltern aktueller, $74 \%$ vollständiger und $64 \%$ verständlicher informiert. Aus der Sicht von Wirtschaftlichkeit und Effizienz kann gezeigt werden, dass der Aufwand von persönlichen (72\%), telefonischen (79\%) 
und schriftlichen (79\%) Absprachen nach Einschätzung der Teilnehmer durch die Anwendung von Smarty gesunken ist.

Schlussfolgerungen: Mit Smarty konnte ein Pilotprojekt für sektorenübergreifende medizinische Versorgung chronisch kranker Kinder mit Mukoviszidose und nach Lebertransplantation mit Aufbau eines Behandlernetzwerkes durchgeführt werden. Durch Smarty kann eine Qualitätsverbesserung in der Patientenversorgung durch bessere Erreichbarkeit der Teilnehmer, verbesserte Datensicherheit bei der Übermittlung von Befunden, Bildern und Berichten und weniger Informationsverlust gezeigt werden. Die Teilnahme der niedergelassenen Kollegen scheiterte teilweise an den technischen Voraussetzungen in den Praxen oder grundsätzlicher Zurückhaltung gegenüber digitaler Kommunikation. Weitere Studien zur Verwendung und Wirtschaftlichkeit der Online-Kommunikation sind nötig.

\section{Poster}

\section{Gastroenterologie}

\section{DGKJ-PO 01}

\section{Ist die hepatische Morbus-Wilson-Verlaufsform rein hepatisch?}

Charlotte Thiels', Anjona Schmidt-Choudhury², Thomas Lücke

${ }^{1}$ Universitätsklinik für Kinder- und Jugendmedizin der Ruhr-

Universität Bochum, Neuropädiatrie und Sozialpädiatrie, Bochum, Deutschland;'²Universitätsklinik für Kinder- und Jugendmedizin der Ruhr-Universität Bochum, Gastroenterologie, Bochum, Deutschland; ${ }^{3}$ Universitätsklinikum der Ruhr-Universität Bochum, Neuropädiatrie und Sozialpädiatrie, Bochum, Deutschland

Einleitung: Der Morbus Wilson (WD, OMIM \#277900) ist eine Erkrankung des Kupferstoffwechsels aufgrund einer Mutation im ATP7B-Gen. Die damit einhergehende gestörte Kupferclearance führt zu einer Kupferbelastung insbesondere von Leber und ZNS. Die Einteilung erfolgt in eine hepatische, neurologische oder psychiatrische Verlaufsform, obschon es sich um eine Multisystemerkrankung handelt. Die klinische Präsentation ist, u. a. abhängig von der stärksten Organmanifestation, sehr variabel. Kinder weisen am häufigsten eine „hepatische“ Manifestation auf. Aufgrund der Fokussierung auf die hepatischen Symptome, insbesondere bei den $25 \%$ Kindern mit schwerer hepatischer Manifestation, liegen kaum Daten vor über neurologische Auffälligkeiten dieser Patienten. Anhand eines Fallberichts weisen wir auf eindeutige und subtile neurologische Symptome einer 9-jährigen Patientin mit hepatischer Manifestation hin. Fallbericht: 2./2 Kindern. Kleinkindalter: expressive Sprachentwicklungsstörung, 8. LJ.: gute kognitive Leistung. 9. LJ: Leistungssport, Schulleistungen schleichend verschlechtert. Im Rahmen eines Infektes mit ausgeprägter Splenomegalie und schwerer Lebersynthesestörung wurde im Verlauf die Diagnose eines WD gestellt und molekulargenetisch bestätigt. Die Patientin wurde für Eurotransplant gelistet und eine cranielle MRT erfolgte. T1: diskrete symmetrische Signalanhebung der basalen Stammganglien unter Einbeziehung der Thalami mit Übergang zur Crus cerebri.

Neurologie bei Diagnosestellung: starke Muskelkrämpfe, Koordinationsprobleme, ein schlechtes Schriftbild und verschlechterte Schulleistungen (Durchschnitt 1 Note schlechter).

Unter konsequenter Entkupferung (D-Penicillamin) rasch komplettes Verschwinden der Muskelkrämpfe und der Koordinationsprobleme, innerhalb eines Jahres Normalisierung der Leberwerte sowie Verbesserung der Schulleistungen. Das Schriftbild ist aktuell gut. 13. LJ: Sprachgedächtnisprobleme und stärker impulsives Verhalten.

Die Veränderungen in der craniellen MRT haben sich innerhalb von 4 Jahren rückgebildet.

Diskussion: Klinische Daten über neurologische Symptome der primär hepatisch erkrankten Kinder liegen kaum vor. Muskelkrämpfe sind in Neurology als neurologisches Symptom bei pädiatrischen WD-Patienten erwähnt. Bislang ist wenig verstanden, warum bei Fehlen von Geno-/ Phänotypkorrelation die Diagnose WD trotz Multisystemerkrankung bei jüngeren Patienten anhand vorwiegend hepatischer Klinik gestellt wird. Bei ATP7b-/- Mäusen erfolgt die cerebrale Kupferablagerung in inverser Korrelation zum Alter. Cranielle MRT (1,5 T) mittels QSM (Quantitive Susceptibility Mapping) zeigen Auffälligkeiten bei symptomatisch-hepatisch, aber neurologisch unauffälligen kindlichen WD im Bereich der Basalganglien. Wir möchten für neurologische (und psychiatrische) Symptome bei pädiatrischen WD-Patienten mit „hepatischem “ Verlauf anhand des Fallberichtes sensibilisieren. Mehr Daten können zum Krankheitsverständnis beitragen. 


\section{DGKJ-PO 02}

\section{Manifestation eines Morbus Crohn mit intestitieller Nephritis}

\section{Lena Gebert, Christian von Schnakenburg, Olaf Raecke}

Klinikum Esslingen, Klinik für Kinder und Jugendliche, Esslingen, Deutschland

Ein 16-jähriges Mädchen stellte sich mit Gewichtsabnahme, Leistungsknick, und chronischer Diarrhoe mit erhöhtem Calprotectin im Stuhl unter dem Verdacht auf einen M. Crohn vor. Bei der Abklärung fiel neben einer Anämie eine stark eingeschränkte Nierenfunktion auf (S-Kreatinin $4,5 \mathrm{mg} / \mathrm{dl}$, GFR $16 \mathrm{ml} / \mathrm{min}$ ), deren Ursache eine chronische, interstitielle Nephritis mit schwerer, globaler (70 von 94 Glomerula) und fokal-segmentaler (3/94) Sklerose war.

Nach Hydrierung fiel das Kreatinin auf minimal 3,5 mg/dl ab. Endoskopisch fanden sich makroskopisch wie mikroskopisch entzündliche Veränderungen i.S. eines M. Crohn mit Manifestation an Ösophagus, Magen und dem gesamten Colon mit Aussparung des Rektums.

Unter der Therapie mit Steroiden, die zur zeitgleichen Behandlung der Nephritis zur Remissionsinduktion eingesetzt wurden, und dem unmittelbaren Beginn mit Azathioprin trat rasch eine anhaltende Remission der chronisch entzündlichen Darmerkrankung ein. Die chronischen Nierenveränderungen zeigten keine Besserung und hatten bereits zu einer Nierenschrumpfung und irreversiblen Schädigung geführt, sodass die Patientin aktuell unter supportiver Therapie mit Vitamin D, Erythropoietin und ACE-Inhibitoren auf eine präemptive Nierentransplantation vorbereitet wird.

Zusammenfassung: Eine interstitielle Nephritis ist eine seltene extraintestinale Manifestation bei M. Crohn vor einer immunmodulatorischen Therapie mit Azathioprin und kann der gastrointestinalen Symptomatik vorausgehen.

\section{Literatur}

1. Corica D, Romano C (2016) Renal Involvement in Inflammatory Bowel Diseases. J Crohns Colitis 10(2):226-235

2. Joyce $E$, Glasner P, Ranganathan S, Swiatecka-Urban A Tubulointerstitial nephritis: diagnosis, treatment, and monitoring. Pediatr Nephrol :

\section{DGKJ-PO 03}

\section{Das Cannabinoid-Hyperemesis Syndrom. Ein Fallbericht.}

\section{Florian du Bois, Hendryk Schneider}

Universitätsklinikum Freiburg, Zentrum für Kinder- und Jugendmedizin, Freiburg, Deutschland

In dieser Kasuistik berichten wir von einem 17-jährigen Jugendlichen mit akutem, rezidivierendem Erbrechen, starker Übelkeit und kolikartigen Bauchschmerzen seit wenigen Stunden. Die Beschwerden traten bereits in der Vergangenheit in Phasen von wenigen Tagen Dauer auf. Es zeigte sich kein Ansprechen auf alle gängigen Antiemetika. Klinisch ergaben sich keine Hinweise auf eine akute neurologische Ursache, laborchemisch konnte eine Infektion ausgeschlossen werden. Ein Ultraschall des Abdomens und eine Ösophagogastroduodenoskopie (ÖGD) blieben ohne Befund. Anamnestisch und im Urin konnte ein hoher Cannabis-Konsum erhoben werden, sodass wir von einem Cannabinoid-Hyperemesis Syndrom (CHS) ausgingen.

Das CHS stellt ein unterdiagnostiziertes, akutes Krankheitsbild, ähnlich dem Cyclic Vomiting Syndrome, dar. Therapeutisch zeigt sich kein Ansprechen auf Antiemetika, in Fallstudien ist eine Symptomlinderung durch heißes Duschen und die topische Anwendung von Capsaicin beschrieben Eine dauerhafte Symptomfreiheit kann letztlich nur durch eine Beendigung des Cannabiskonsums erzielt werden. Das klinische Management sollte mit der akuten Rehydratation und Antiemese z. B. mit Haloperidol oder topischem Capsaicin beginnen. Mittels einer orientierenden Laborkontrolle, eines Abdomen Ultraschalls, einer ÖGD, und bei begründetem Verdacht zusätzlich Röntgen- oder CT-Abdomen, sollten relevante Differentialdiagnosen zu Beginn ausgeschlossen werden. Eine gründliche Anamnese kombiniert mit einem Drogenscreening im Urin kann schließ- lich den entscheidenden Hinweis geben. Eine frühe Anbindung an eine Drogenentwöhnungs-Stelle sollte erfolgen.

\section{DGKJ-PO 04}

\section{Intragastrale Sprengung einer Knopfbatterie nach Ingestion}

Cristina Schwerdtfeger', Moritz Meißner ${ }^{2}$, Stephan Stöhrer², Susanne Lohmar' ${ }^{2}$, Marcus Pauly ${ }^{2}$

'Siegburg, Deutschland;'² Asklepios Kinderklinik Sankt Augustin, Abteilung für Kinderchirurgie, Sankt Augustin, Deutschland

Hintergrund: Das Verschlucken von Fremdkörpern stellt in der Kinderund Jugendmedizin ein weit verbreitetes Problem dar. Besonderen Stellenwert hat hierbei die Ingestion von Knopfbatterien. Neben der notfallmäßigen Extraktion, die nach Steckenbleiben aller Fremdkörper im Ösophagus indiziert ist, sollten Knopfbatterien wegen drohender Schleimhautläsionen durch elektrische Entladung auch zeitnah (innerhalb $24 \mathrm{~h}$ ) aus dem Magen entfernt werden.

Fallbericht: Wir berichten über ein 3-jähriges Mädchen, das die entladene Knopfbatterie aus einem Werbeartikel verschluckt hatte. Die Eltern berichteten über einen lauten Knall aus dem Bauch des Kindes wenige Minuten später, woraufhin sofort die notfallmäßige Vorstellung in unserer Klinik erfolgte. Das Kind klagte über leichte Bauchschmerzen, die körperliche Untersuchung ergab keine Auffälligkeiten, die Vitalparameter waren stabil. In den umgehend veranlassten Röntgenaufnahmen von Thorax und Abdomen zeigten sich mehrere strahlendichte Strukturen in Projektion auf den Magen, Hinweise auf ein Pneumothorax oder -peritoneum bestanden nicht. Es erfolgte die sofortige stationäre Aufnahme zur endoskopischen Fremdkörperbergung und zum Ausschluss explosionsbedingter Begleitverletzungen. Intraoperativ zeigten sich zwei Scheimhautläsionen im Magen, eine Perforation konnte ausgeschlossen werden. Das ca. $1 \mathrm{~cm}$ große Hauptfragment konnte aus dem Speisebrei des Magens geborgen werden, ein weiteres großes Fragment projizierte sich im Verlauf deutlich lageverändert zunächst im Duodenum und später im Jejunum und konnte endoskopisch nicht geborgen werden. Postoperativ erfolgte zunächst die intensivmedizinische Überwachung der extubierten und kreislaufstabilen Patientin. Am 01.04. wurde nach Klysmagabe das zweite Fragment via naturalis ausgeschieden. Eine Kontroll-ÖGD am 04.04. zeigte intakte Schleimhautverhältnisse ohne weitere Verletzungsfolgen.

Schlussfolgerung: Neben den bereits bekannten Komplikationen nach Ingestion von Knopfbatterien (Entstehung von Drucknekrosen, physikalische Schäden durch Stromfluss) zeigt unser Beispiel zudem weitere zeitnah drohende Gefahren durch Detonation bis zur möglichen Hohlorganperforation. Deshalb sollte auch bei intragastraler Lage von Knopfbatterien eine frühzeitige Indikation zur Endoskopie gestellt werden.

\section{DGKJ-PO 05}

\section{Ergebnisse eines kohortenbasierten Elternfragebogens von Kindern mit Dreimonatskoliken}

Jochen Kittel', Christian Apfelbacher', Birgit Seelbach-Göbel', Susanne Brandstetter', Michael Kabesch ${ }^{4}$, Lena Nonnenmacher', Michael Melter ${ }^{4}$ ${ }^{1}$ Kinder Uniklinik Ostbayern (KUNO), Universität Regenesburg, Klinik St. Hedwig, Neonatologie, Regensburg, Deutschland; ${ }^{2}$ Universität Regensburg, Medizinische Soziologie, Institut für Epidemiologie und Präventivmedizin“ Regensburg, Deutschland;'Klinik für Gynäkologie und Geburtshilfe, Klinik St. Hedwig, Regensburg, Deutschland; " Klinik für Kinder- und Jugendmedizin, KUNO Klinik St. Hedwig, Regensburg, Deutschland

Einleitung: Dreimonatskoliken (DMK) beschreiben Schreien, Weinen und Quengeln ohne erkennbare Ursache bei gesunden, gedeihenden Säuglingen (1). Diagnostiziert werden die DMK nach Wessel bzw. modifizierten Wessel Kriterien. Das Problem betrifft viele Familien und ist für 10-20 \% aller Kinderarztbesuche bei Säuglingen zwischen zwei Wochen und drei Monaten (2). Ziel unserer Arbeit ist es, die Häufigkeit von Arztbesuchen aufgrund von DMK in unserer Geburtskohorte zu ermitteln, zudem sollen 
medikamentöse und nicht-medikamentöse Massnahmen für die Behandlung der DMK erfasst werden.

Methodik: In der Arbeit wurden Daten aus einer Geburtskohorte (KUNO Kids Gesundheitsstudie) analysiert (5). Allen Eltern, die 4 Wochen nach Geburt des Kindes in einem Fragebogen einzelne Kriterien nach Wessel für DMK berichteten, wurde erneut ein Fragebogen mit 29 Fragen mit Fragen rund um DMK zugesendet. Die Auswertung erfolgt deskriptiv.

Ergebnisse: Von 196 Kindern lag der DMK Fragebogen vor, das Alter der Kinder lag im Durchschnitt bei 18 Wochen.

86/196 (44,1 \%) waren weiblich. Das mittlere Geburtsgewicht lag bei 3373 g. Die Geburt fand bei $12(6,3 \%)$ Kindern vor der Vollendung der 37. Schwangerschaftswoche statt. Mit Hilfe einer primären Sectio kamen 47/196 zur Welt (24\%), mit sekundärer Sektio bzw. vaginal operativ 28/196 (14\%), 121 (72,0 \%) Kinder wurden vaginal entbunden.

Bei 91/196 Kinder gab es zum Zeitpunkt der Bearbeitung Hinweise auf die Dreimonatskoliken nach Wessel, hier wurden weitere Fragen ausgewertet Aufgrund der Symptome waren 38/91 (42 \%) der Eltern mit ihrem Kind bei einem Kinderarzt.

Dort wurden verschiedene Diagnosen für das Verhalten genannt, v. a. Dreimonatskoliken (15/38) und Blähungen (7/38).

Die DMK war Anlass für Ernährungsumstellungen bei Mutter und/oder Kind.

Mütter verzichteten auf blähende Nahrungsmittel (11/91), auch auf laktosehaltige Nahrung (8/91).

Bei den Kindern wurden v. a. ein Wechsel der Babynahrung (14/91) durchgeführt.

Eltern berichteten von medikamentösen Therapieversuchen, v.a. simeticonhaltige Arzneimittel (19/91) und homöopathische Therapien (10/91) und Inanspruchnahme alternativer Therapien (Osteopathie (32/91), Babymassage (13/91) und Ergo-/Physiotherapie/Manuelle Therapie (4/91)). Diskussion: Eltern von Kindern mit DMK haben einen erhöhten Betreuungsbedarf, was sich in häufiger Inanspruchnahme von Kinderärzten zeigt. Oft werden dort den Eltern verschiedene Diagnosen für das Verhalten der Kinder genannt, was eine Verunsicherung der Eltern bewirken kann. Den Eltern werden therapeutische Optionen wie simeticonhaltige Arzneimittel oder homöopathische Therapien angeboten, was dann automatisch einen Krankheitswert impliziert. Eltern sind bereit, alternative Therapien wie Osteopathie, Babymassage oder manuelle Therapie beim Kind zu testen.

Zudem kommt es in der Folge zu Ernährungsumstellungen bei Mutter und Kind.

Als Folge der eigentlich harmlosen DMK kommt es an verschiedenen Stellen zu vielerlei Maßnahmen.

\section{Literatur}

1. Benninga MA, Nurko S, Faure C, Hyman PE, Roberts Schechter StJINL (2016) Childhood functional gastrointestinal disorders: Neonate/toddler. Baillieres Clin Gastroenterol 150(6):1443-1455e. https://doi.org/10.1053/j.gastro.2016.02.0

\section{DGKJ-PO 06}

Alimentärer Vitamin C-Mangel und schwerer Eisenmangel durch Fehlernährung bei Kleinkindern mit Migrationshintergrund

Barbara Naust, Lea Piepenbrink, Antonia Bauer, Rosa Wördehoff, Georg Hillebrand

Klinikum Itzehoe, KLinik für Kinder- und Jugendmedizin, Neonatologie und Pädiatrische Intensivmedizin, Itzehoe, Deutschland

Kasuistik 1: 14 Monate altes Mädchen, die Familie stammt aus Afghanistan. Seit 3 Wochen entzündliche Hauterkrankung und Blässe. Bei Aufnahme ausgeprägt blasses Hautkolorit und blasse Schleimhäute, Tachypnoe und Tachykardie, Systolikum.

Labor: Schwere Eisenmangelanämie (Hb 1,9 g/dl, Hkt: 9 \%; MCV: 49 fl, LDH 320U/l, Eisen 13,1 $\mu \mathrm{g} / \mathrm{dl}$, Ferritin < $5 \mu \mathrm{g} / \mathrm{dl}$, Transferrin $411 \mathrm{mg} / \mathrm{dl}$, Transferrinsättigung 3,3 \%, Hämoglobin-Elektrophorese: unauffällig, Vitamin D: $9 \mu \mathrm{g} / \mathrm{l}(>30)$, Vit. B12: $1340 \mathrm{ng} / 1$ (410-1210), Folsäure: $2,2 \mu \mathrm{g} / \mathrm{l}$
(14-52), Vitamin C: $<1,0 \mathrm{mg} / \mathrm{l}(5,0-15,0)$, Vit. E: 9,4 mg/l (5,0-18,0), Vit. A: $308 \mu \mathrm{g} / \mathrm{l}(300-700)$, Zink: 0,5 mg/l $(0,2-1,1)$

Transfusion eines Erythrozytenkonzentrates. Genauere Ernährungsanamnese: 2 Monate Muttermilch, dann Formulaernährung. Seit dem 7. Lebensmonat fast ausschließlich Kuhmilch (H-Vollmilch) und nur alle 2-3 Wochen 1-2 Teelöffel Gemüse/Fleisch/Reis.

Substitution von Eisen, Vit. C, Eisen, Folsäure und Vit. D. Beratung der Eltern. Hierunter zunächst Normalisierung der Werte, nach Beendigung der Substitution erneut Vit. C-Mangel. Umsetzung einer altersentsprechenden Ernährung im häuslichen Umfeld weiterhin schwierig. Aktuell sozialmedizinische Nachsorge verordnet.

Kasuistik 2: 3 Jahre alter Junge. Eltern stammen aus Syrien. Einweisung zur Abklärung motorische Entwicklungsverzögerung. Auch hier ausschließliche Kuhmilchernährung.

Labor: Hb 9,8 g/dl, Hkt:31 \%; MCV:59 fl; Eisen 13,1 $\mu \mathrm{g} / \mathrm{dl}$, Ferritin $6 \mu \mathrm{g} /$ dl, Transferrin $373 \mathrm{mg} / \mathrm{dl}$, Transferrinsättigung 3,4\%, Vit. D: $22 \mu \mathrm{g} / \mathrm{l}$ (>30), Vit. B12: $1296 \mathrm{ng} / \mathrm{l}$ (410-1210), Folsäure: 1,5 $\mu \mathrm{g} / \mathrm{l}$ (14-52), Vita$\min \mathrm{C}<1,0 \mathrm{mg} / \mathrm{l}(5,0-15)$

Normalisierung der Werte unter Substitution und adäquater Ernährung, bessere motorische Entwicklung.

Diskussion: In beiden Fällen bestand neben einer Eisenmangelanämie ein ausgeprägter alimentärer Vitamin-C-Mangel durch schwere Fehlernährung, jedoch ein weitgehend perzentilengerechtes Wachstum.

Der alimentäre Vitamin C-Mangel (Möller-Barlow-Krankheit) ist ein extrem seltenes Krankheitsbild, das meist infolge Fehlernährung bei psychiatrischen Störungen beobachtet wird. Das Vollbild der Erkrankung (Skorbut) war in unseren Fällen noch nicht ausgeprägt, allerdings zeigte Kind 1 bereits Wundheilungsstörungen. In unserer Klinik beobachteten wir in den letzten Jahren mehrere Kinder mit schwerer Fehlernährung (meist ausschließliche Kuhmilchernährung bei Kleinkindern) und resultierendem frühkindlichem Vit. C Mangel. Aufgrund von Sprachbarrieren fehlt Familien mit Migrationshintergrund häufig der Zugang zu Informationen zu gesunder Kinderernährung. Bei noch gutem Gedeihen werden diese "Milchtrinker" oft in der normalen Vorsorgeroutine nicht erkannt. Aktuelle EU-Richtlinien führen zudem zu sinkendem Gehalt an Vit. C in Fruchtsäften, die bisher möglicherweise als ausreichende Quelle angesehen wurden.

Das Vorliegen eines kindlichen, alimentären Vit. C-Mangels sollte bei entsprechenden Risiko-Konstellationen auch in Regionen mit vermeintlich guter Zufuhr in Erwägung gezogen werden.

\section{DGKJ-PO 07}

\section{Eosinophile Gastroenterokolitis mit transienter exokriner Pankreasinsuffizienz und sepsis-ähnlichem Krankheitsbild bei einem 2 Monate alten Säugling}

Yvonne Zeißig', Daniela Aust'², Martin W. Laaß'

'Universitätsklinikum Carl Gustav Carus an der Technischen Universität Dresden, Klinik und Poliklinik für Kinder- und Jugendmedizin, Dresden, Deutschland; ${ }^{2}$ Universitätsklinikum Carl Gustav Carus an der Technischen Universität Dresden, Institut für Pathologie, Dresden, Deutschland

Hintergrund: Nicht-IgE vermittelte Nahrungsmittelallergien umfassen ein breites Spektrum an Gastroenteropathien mit teils überlappender Symptomatik von variabler Ausprägung. Hierunter fallen auch die eosinophilen Enteropathien mit der Proktitis als häufigste Form, welche sich meist in den ersten Lebensmonaten mit Hämatochezie bei sonst unbeeinträchtigtem Säugling manifestiert. Systemische Manifestationen mit ausgedehnterem Befall des Gatrointestinaltraktes sind selten.

Fallbericht: Wir berichten über ein 2 Monate altes Mädchen, welches mit gastroenteritischer Symptomatik und febrilen Temperaturen auf unsere Intensivstation aufgenommen wurde. Weiterhin bestanden erhöhte Entzündungsparameter, eine Tachykardie, eine verlängerte Rekapillarisierungszeit, sowie eine verminderte Diurese, so dass eine antibiotische Therapie mit Ampicillin und Cefotaxim begonnen wurde. Nach Ausschluss einer bakteriellen Infektion bei negativen Blut-, Liquor- und Urinkulturen, sowie unauffälligen Stuhlbefunden wurde diese nach 5 Tagen wieder 
beendet und das Kind in stabilem Allgemeinzustand auf die Normalstation verlegt.

Hier entwickelte das Mädchen dann zunehmend blutige Stühle und Ödeme bei anhaltender Hypalbuminämie, so dass das Kind mehrfach intravenös Humanalbumin erhalten musste. Im Stuhl fand sich ein deutlich erhöhtes Calprotectin (>2100 $\mu \mathrm{g} / \mathrm{g}$ Stuhl), sowie eine nicht nachweisbare bzw. stark erniedrigte pankreatische Elastase im Sinne einer exokrinen Pankreasinsuffizienz. Das CF-Neugeborenenscreening ergab einen negativen Befund. Im Schweißtest zeigten sich ebenfalls keine Auffälligkeiten. Sonografisch stellte sich das Pankreas unauffällig dar. Bei mangelnder Gewichtszunahme wurde eine Kreon-Substitution begonnen.

Bei Verdacht auf eine Nahrungsmittelallergie begannen wir eine kuhmilchfreie Ernährung der Mutter, welche jedoch keinen anhaltenden Effekt erbrachte. Wir entschlossen uns daher zur Durchführung einer Gastro- und Koloskopie. Makroskopisch sahen wir dabei im Rektum, Sigma und Colon descendens eine entzündlich veränderte Schleimhaut mit fleckförmigen Rötungen. Histologisch zeigte sich eine mäßiggradig aktive, vermehrt eosinophilenreiche Entzündungsreaktion im gesamten untersuchten Gastrointestinaltrakt. Nach Umstellung der Ernährung auf eine aminosäurebasierte Spezialnahrung traten keine blutigen Stühle mehr auf, die Gewichtsentwicklung war zufriedenstellend und die pankreatische Elastase im Stuhl normalisierte sich, so dass die Kreon-Substitution beendet werden konnte.

Zusammenfassung: Aufgrund der endoskopischen und histologischen Befunde, sowie der Besserung der Symptomatik nach Umstellung auf eine aminosäurebasierte Nahrung stellten wir die Diagnose einer eosinophilen Gastroenterokolitis mit schwerer Resorptionsstörung und Hypalbuminämie, sowie transient erniedrigter pankreatischer Elastase.

\section{DGKJ-PO 08}

\section{Niemann-Pick-Disease-C (NPD-C): Untersuchung der} Chitotriosidase und Oxysterole bei Säuglingscholestase

Frieda Sarif', Antje Ballauff', Thorsten Marquardt ${ }^{3}$

${ }^{1} \mathrm{HELIOS}$ Klinikum Krefeld, Kinder- und Jugendmedizin, Krefeld,

Deutschland; ${ }^{2}$ Helios Klinikum Krefeld, Kinder- und Jugendmedizin,

Kindergastroenterologie und -hepatologie, Krefeld, Deutschland;

${ }^{3}$ Universitätsklinikum Münster, Kinder- und Jugendmedizin, Angeborene

Stoffwechselerkrankungen, Münster, Deutschland

Hintergrund: Die NPD-C gehört zu den lysosomalen Lipidspeicherkrankheiten mit autosomal-rezessivem Erbgang. Mutationen im NPC1-(95\%) oder NPC2-Gen (5\%) führen zu einem Enzymdefekt des intrazellulären Cholesterintransports bzw. der -speicherung mit Ansammlung von Sphingolipiden und Cholesterol in den Endosomen/Lysomen und Degeneration der Zellen. Betroffen sind insbesondere Leber, Milz und das Nervensystem. Das Manifestationsalter sowie die klinische Ausprägung sind sehr variabel. Die hepatische Manifestation reicht von Transaminasenerhöhung, Hepatosplenomegalie, zu Säuglingscholestase bis Leberversagen im Säuglingsalter. Bei sehr früher Manifestation ist mit einer schweren neurodegenerativen Erkrankung zu rechnen. Eine erhöhte Chitotriosidaseaktivität ist hinweisend. Die weiterführende Diagnostik war bisher kompliziert und zeitaufwendig.

Fallbericht: Bei einem 5 Wochen alten männlichen Säugling fielen bei der Leistenhernien-OP ein Haut- und Sklerenikterus, eine Dystrophie sowie eine milde Splenomegalie auf. Die Diagnostik ergab eine direkte Hyperbilirubinämie, erhöhte Leberenzyme sowie sonografisch eine Hepatosplenomegalie mit mildem Aszites. Bei unauffälliger Stuhlfarbe bestand kein Hinweis auf eine Gallengangsatresie. Als primäre Behandlung bei Cholestase und Gedeihstörung wurde mit der Substitution fettlöslicher Vitamine sowie MCT-haltiger hochkalorischer Nahrung begonnen. Die weitere Diagnostik blieb ohne wegweisenden Befund, sodass die Stoffwechseldiagnostik erweitert wurde. Die Aminosäuren im Plasma und organischen Säuren im Urin zeigten unspezifische Veränderungen bei Hepatopathie. Das Acylcarnitinprofil sowie die Transferrinelektrophorese waren unauffällig. Als mögliches Zeichen einer lysosomalen Speichererkrankung zeigte sich eine deutlich erhöhte Chitotriosidaseaktivität im Plasma. Durch den Nachweis erhöhter Oxysterole (Trockenblut) ergab sich die Diagnose NPD-C mit dann genetischem Nachweis einer Compound heterozygoten Mutation im NPC1-Gen. Es wurde eine Therapie mit Miglustat begonnen. Hierunter Diarrhoen mit Besserung durch zucker- und v. a. fructosearme Diät. Im Verlauf besserten sich die Leberwerte und die Hepatosplenomegalie. Der Patient wächst entlang der 3. Perzentile, zeigt jedoch eine verzögerte motorische Entwicklung.

Schlussfolgerung: Bei Patienten mit prolongiertem Ikterus neonatorum muss eine Bilirubindifferenzierung erfolgen. Bei Nachweis einer Cholestase dann weitergehende Diagnostik. Die Chitotriosidaseaktivität kann wegweisend sein für eine Speichererkrankung. Neuerdings können unkompliziert im Trockenblut Oxysterole bestimmt werden (www.npc-test.de), sodass diese Diagnostik recht früh in der weiterführenden Cholestaseabklärung erfolgen kann, um weitere therapeutische Schritte einzuleiten. 


\section{Hämato-/Onkologie/Rheuma}

\section{DGKJ-PO 09 \\ Wer suchet, der findet: Seltene Tumorerkrankungen im Kindes- und Jugendalter}

Felicitas Hippert ', I. B. Brecht² , B. Bernbeck', M. Ebinger'2, Michael Ost ${ }^{2}$, D. T. Schneider ${ }^{1}$

'Klinikum Dortmund Klinikzentrum Mitte, Pädiatrie, Dortmund, Deutschland; ${ }^{2}$ Universitätsklinik für Kinder- und Jugendmedizin Tübingen, Hämato-Onkologie, Tübingen, Deutschland

Fragestellung: Seltene Tumoren (ST) im Kindes- und Jugendalter haben eine so extrem niedrige Inzidenz, dass sie bislang nicht in das klinische und wissenschaftliche Netzwerk der Kinderonkologie integriert waren Die Datenlage ist sehr begrenzt und einheitliche Behandlungsstrategien fehlen. Somit sind ST klassische Orphan Diseases. Die Gründung der Arbeitsgruppe für seltene Tumorerkrankungen in der Pädiatrie (STEP) hatte zum Ziel, Strukturen für eine möglichst vollständige Erfassung aller ST aufzubauen. Außerdem wurden ein Konsiliarnetzwerk aufgebaut und Behandlungsempfehlungen für verschiedene ST entwickelt. Hier berichten wir über die Patientenrekrutierung während der ersten 10 Jahre der STEP Arbeitsgruppe.

Material und Methoden: Die Datenerfassung erfolgte von 2008 bis 2018. Erfasst wurden ST bei Patienten bis zum 18. Lebensjahr, die von keiner anderen Therapieoptimierungsstudie oder Register der GPOH erfasst wurden. Bis 2012 wurden alle kinderonkologischen Zentren regelmäßig retrospektiv nach neu diagnostizierten ST befragt. Seit 2012 werden ST prospektiv im STEP Register erfasst. Das Projekt wurde von den Ethikkommissionen der Universitäten in Düsseldorf und Erlangen positiv begutachtet.

Ergebnisse: Insgesamt wurden 624 Patienten mit ST registriert. Am häufigsten wurden Tumoren der Haut (154), des Gastrointestinaltraktes (104), des muskuloskelettalen Systems (90), der Gonaden (84), sowie des HNOBereichs (65) gemeldet. Unter den Hauttumoren sind die Melanome die am häufigsten auftretende Tumorentität (18\% maligne, $18 \%$ spitzoide, 38 \% nicht näher spezifizierte Melanome). Bei den Tumoren des GI-Traktes überwiegen Kolonkarzinome ( $33 \%$ ) und pseudopapilläre Tumoren des Pankreas (20 \%). Die Riesenzelltumoren stellen die häufigsten (29\%) muskuloskelettalen Tumoren dar. Bei den seltenen gonadalen Tumoren überwiegen die juvenilen Granulosazelltumoren (30 \%), Zystadenome (21 \%) und Sertoli-Leydig-Zell-Tumoren (20\%). Die höchste Mortalität zeigen die extrem seltenen NUT-Karzinome. Die Zahl der jährlichen Meldungen stieg über den Erfassungszeitraum an. In den Jahren 2008-2014 schwankten die Fallzahlen um 40 Meldungen pro Jahr. Seit 2015 liegt die Zahl der jährlichen Meldungen bei ca. 90 .

Diskussion: Mit der Etablierung zentraler Strukturen für die klinische Beratung und Dokumentation von ST steigt die Erfassungsrate. Als Gesamtgruppe sind seltene Tumoren vergleichbar häufig wie andere klassische kinderonkologische Tumoren. Dabei sind die ST hinsichtlich Lokalisation, Histologie und Prognose extrem heterogen; jede einzelne Diagnose wird in Deutschland meist seltener als $10 \mathrm{Mal}$ im Jahr gestellt. Durch die zentrale Erfassung und Analyse dieser Erkrankungen, gemeinsam mit internationalen Partnern, kann es gelingen, Behandlungsstrategien für ST zu entwickeln und damit die Behandlungsqualität erheblich zu verbessern. Unterstützt durch die Deutsche Kinderkrebsstiftung und die Madeleine Schickedanz KinderKrebs-Stiftung

\section{DGKJ-PO 10 \\ Ex vivo Cytokine Kinetics under bacterial stimulation in Febrile Neutropenic Children}

Mira Siegmund', Julia Pagel', Tasja Scholz', Jan Rupp², Christoph Härtel', Melchior Lauten ${ }^{1}$

'Universitätsklinikum Schleswig-Holstein, Campus Lübeck, Klinik für Kinder- und Jugendmedizin, Lübeck, Germany; ${ }^{2}$ Universitätsklinikum Schleswig-Holstein, Campus Lübeck, Klinik für Kinder- und Jugendmedizin; Klinik für Infektiologie und Mikrobiologie; Deutsches Zentrum für Infektionsforschung (ZDIF) Hamburg - Lübeck - Borstel - Riems, Lübeck, Germany

Febrile neutropenia is a common and serious complication during therapy of childhood cancer. Empirical, broad-spectrum, intravenous antibiotics are usually administered until fever declines and the neutrophil cell count normalizes. However, biomarkers for early detection of infections and information on cytokine profiles to differentiate between different clinical courses of febrile neutropenia are scarce.

The underlying hypothesis of our study was that distinct cytokine patterns can distinguish between different courses of febrile neutropenia in pediatric oncology patients and lead to individualized risk stratification protocols during the first days of the episodes. In addition, we hypothesized that stimulated samples may allow a clearer separation of different risk groups of patients than unstimulated samples.

We conducted a retrospective study in 47 neutropenic fever episodes of 33 pediatric oncology patients to assess profiles of 27 different cytokines. Blood samples were collected at days $0,1,4,7,10$, and 14 from the onset of fever and stimulated ex vivo with lipopolysaccharides (LPS) and Staphylococcus epidermidis (SE) for $24 \mathrm{~h}$. The cytokine levels were measured in the plasma by a multiplex immunoassay system (Bio-Plex Pro ${ }^{\text {sn }}$ Human Cytokine 27-plex Assay).

During the episodes of febrile neutropenia most of the cytokines show an increase in concentration. Already at day 1 of the episodes unstimulated, LPS and SE stimulated samples show lower concentrations of IL-8, MIP$1 \alpha$, MIP- $1 \beta$, MCP- 1 and TNF- $\alpha$ in children with severe courses. There are no remarkable differences between males and females.

Future studies may focus on validating cytokine profiles as possible biomarkers to differentiate between infectious and non-infectious febrile neutropenia episodes. In addition, MIP-1 $\alpha$, MIP-1 $\beta$, IL-8, MCP-1 and TNF- $\alpha$ might detect early severe cases of febrile neutropenia and might, therefore, add to a rational use of antibiotics and a reduction of time of hospitalization.

\section{DGKJ-PO 11}

\section{Eosinophilie als Erstmanifestation der akuten lymphatischen} Leukämie

Kyriaki Papaioannou', Sonja Trepels-Kottek², Mark Schoberer', Ulrich Pohlmann ${ }^{3}$, Jaime F. Vazquez-Jimenez ${ }^{4}$, Gunter Kerst ${ }^{5}$, Olga Moser' ${ }^{7}$ Udo Kontny', Lisa Lassay'

'Uniklinik RWTH Aachen, Sektion Pädiatrische Hämatologie, Onkologie und Stammzelltransplantation, Klinik für Kinder- und Jugendmedizin, Aachen, Deutschland;'Znniklinik RWTH Aachen, Sektion Neonatologie und Intensivmedizin, Klinik für Kinder- und Jugendmedizin, Aachen, Deutschland: ${ }^{3}$ St. Marien-Hospital Düren, Klinik für Kinder- und Jugendmedizin, Düren, Deutschland; ${ }^{4}$ Uniklinik RWTH Aachen, Kinderherzchirurgie, Aachen, Deutschland; ${ }^{5}$ Uniklinik RWTH Aachen, Klinik für Kinderkardiologie, Aachen, Deutschland

Anamnese: Es erfolgte die Vorstellung eines 8-jährigen Jungen mit seit 6 Wochen bestehenden fast täglichen Fieberschüben bis $39^{\circ} \mathrm{C}$, begleitet von einem flüchtigen petechialen Exanthem, sowie rezidivierenden Episoden von Nachtschweiß. Ansonsten bestand keine weitere Symptomatik und keine Vorerkrankungen.

Diagnostik: Der somatische Untersuchungsbefund war unauffällig. Laborchemisch fielen eine ausgeprägte Leukozytose $(25.000 / \mu \mathrm{l})$ und schwere Eosinophilie (62\%) auf. Ansonsten zeigten sich keine Blutbildauffälligkeiten. 
In der durchgeführten Diagnostik gab es keine Hinweise auf eine allergische, parasitäre oder rheumatische Erkrankung. Zum Ausschluss einer malignen Erkrankung wurde eine Knochenmarkpunktion durchgeführt. Hier zeigte sich das Bild einer akuten Leukämie mit einem Blastenanteil von $31 \%$ und begleitender Eosinophilie ( $25 \%)$. Immunphänotypisch konnte eine Vorläufer B-ALL diagnostiziert werden. In der Zytogenetik konnten keine für eine ALL oder ein hypereosinophiles Syndrom typischen Aberrationen festgestellt werden.

Verlauf und Therapie: Im Weiteren wurde die ALL-Therapie gemäß der Studie AIEOP-ALL-BFM 2017 eingeleitet. Bei einem Blastenanteil von $18 \%$ im Knochenmark an Tag +15 erfolgte die Zuordnung in die Hochrisikogruppe, an Tag 33 wurde die Remission der Grunderkrankung erreicht. Unter Therapie zeigte sich innerhalb von 18 Tagen eine vollständige Rückbildung der Eosinophilie sowohl im peripheren Blutbild als auch im Knochenmark des Patienten. Zusätzlich entwickelte er a. e. im Rahmen der ausgeprägten Eosinophilie eine Löffler Endokarditis, welche in einen Mitralklappenersatz und die Einleitung einer Vollheparinisierungstherapie resultierte.

Diskussion: Eine Eosinophilie kann selten bei Kindern im Rahmen von Neoplasien auftreten v.a. als primäre-klonale Eosinophilie aber auch als eine reaktive Eosinophilie. Die akute lymphatische Leukämie mit begleitender Eosinophilie ist eine sehr seltene Entität mit nur wenigen Fallberichten in der Literatur. Die Eosinophilie im Rahmen der ALL wird als reaktives Phänomen angesehen und als Ursache wird eine hohe Zytokinproduktion (v.a. Interleukin-3 und Interleukin-5) durch die leukämischen Blasten vermutet. In ca. $20 \%$ der Fälle gelingt der molekulargenetische Nachweis der Translokation $\mathrm{t}(5 ; 14)(\mathrm{q} 31 ; \mathrm{q} 32)$, welche zu einer Überexpression des Interleukin-3-Gens führt und somit zur Proliferation und Aktivierung der eosinophilen Granulozyten. Oft entwickeln die Patienten im Laufe der Erkrankung Organschäden aufgrund der eosinophilen Toxizität, was ihre Prognose deutlich verschlechtert.

Fazit: Im Kindesalter sind die häufigsten Ursachen einer Eosinophilie Infektionen (v.a. parasitäre Erkrankungen) und allergische Erkrankungen. Eine Eosinophilie kann aber selten auch im Rahmen von Neoplasien wie z.B. ALL auftreten. Eine persistierende Eosinophilie führt zu schweren Organschädigungen deshalb ist in der Diagnostik ein rationelles und systematisches Vorgehen gefragt.

\section{DGKJ-PO 12}

\section{Kleinkind mit JMML und klinischen Zeichen einer NF1 ohne} Nachweis einer familären oder sporadischen Keimbahnmutation

\section{Katja Storch}

\section{Abteilung für Neuropädiatrie, Dresden, Deutschland}

Hintergrund: Die juvenile myelomonozytäre Leukämie (JMML) ist eine seltene Form des Myeloproliferativen Syndroms (MPS) im Kindesalter (1). Betroffen sind vorwiegend Jungen im Alter von zwei Jahren mit Initialsymptomen einer Hepatosplenomegalie (HSM) und Leukozytose. Sie ist assoziiert mit Neurofibromatose Typ 1 (NF1) aufgrund einer Mutation im NF-1 Gen, dessen Genprodukt Neurofibromin ein Antagonist des ProtoOnkogens RAS ist. Die NF1 unterliegt einer Spontanmutationsrate von $50 \%$ bei vollständiger Penetranz (2).

Im Gegensatz zur autosomal-dominanten, krankheitsverursachenden heterozygoten Keimbahnmutation der NF1, liegt bei der JMML eine zweite somatische Mutation als Second-Hit vor, die die Inhibierung von RAS durch Fehlen von Neurofibromin verhindert (3).

Fall: Wir berichten über einen zweijährigen Jungen mit Erstdiagnose einer JMML im Alter von neun Monaten und Initialsymptomatik einer persistierenden Bronchitis, Blässe, HSM und Leukozytose. Es bestanden ein Makrozephalus, Hypertelorismus, kraniale Xanthogranulome, sowie vereinzelte Café-au lait Flecken. Die Knochenmarkpunktion ergab einen Blastenanteil von $30 \%$ bei unauffälliger Immunphänotypisierung. Genetisch konnte eine charakteristischen Monosomie 7, Deletion 7q bzw. Trisomie 8 sowie BCR-ABL Fusionsgen nicht nachgewiesen werden. Die Diagnose einer JMML wurde schließlich mittels Lymphknotenhistologie und Hautbiopsie gesichert durch Nachweis einer Vermehrung aggregierter, reifer plasmazytoider dendritscher Zellen (MPDCPs), wie sie in Assoziation zu myeloischen Neoplasien auftreten, sowie monozytär differenzierte kutaner Zellinfiltrate. Im Rahmen der Diagnostik auf Rasopathien wurde eine somatische Mutation im Exon sieben und acht des NF1 Gens nachgewiesen. Eine Keimbahnmutation wurde weder beim Patienten, noch bei Eltern und Geschwisterkind gefunden, wobei bei Letzterem multiple Café-au lait Flecken ohne zusätzliche klinische Zeichen einer NF1 zu eruieren waren. Diskussion: Kinder mit NF1 besitzen eine Prädisposition für Malignome. Die Trias NF1, Xanthogranulome und JMML sprechen für eine NF1-assoziierte Genese der JMML $(4,5)$.

Die Diagnose der NF1 kann bereits klinisch anhand der Kriterien des National Institutes of Health (NIH) Consensus gestellt werden. Die Schwierigkeit im Kindesalter besteht in der schrittweisen Manifestation, beginnend mit in Größe und Zahl zunehmenden Café-au lait Flecken, axillärem Freckling, Lisch-Knötchen und Neurofibromen. Molekulargenetisch gelingt der Nachweis in 95 \% (3). In den übrigen Fällen kann entweder eine segmentale NF1 aufgrund eines postzygotischen Mosaikes oder eine Mikrodeletion ursächlich sein. Aufgrund klinischer Zeichen einer NF1 beim Patienten und familiär, wird trotz bisher fehlendem Mutationsnachweis der Keimbahn eine NF1-assoziierte JMML vermutet und eine weiterführende genetische Diagnostik angeschlossen. Da 10-14\% der Kinder mit JMML eine zugrunde liegende NF1 Mutation tragen (3), muss an JMML als Erstmanifestation einer NF1 gedacht werden.

\section{DGKJ-PO 13}

\section{Einseitiger Pleuraerguss - Erstsymptom einer extramedullären AML mit Myelonkompression bei einem Säugling}

Angelina Beer 1 , Ricardo Beck², Malte von Bonin ${ }^{3}$, Jörn Meinel', Sebastian Brenner', Ralf Knöfler' ${ }^{2}$, Julia Hauer ${ }^{2}$

'Universitätsklinikum Carl Gustav Carus Dresden, Klinik und Poliklinik für Kinder- und Jugendmedizin, Fachbereich Neonatologie und Pädiatrische Intensivmedizin, Dresden, Deutschland; ${ }^{2}$ Universitätsklinikum Carl Gustav Carus Dresden, Klinik und Poliklinik für Kinder- und Jugendmedizin,

Fachbereich Pädiatrische Hämatologie/Onkologie, Dresden, Deutschland; ${ }^{3}$ Universitätsklinikum Carl Gustav Carus Dresden, Medizinische Klinik und Poliklinik I, Dresden, Deutschland; ${ }^{4}$ Universitätsklinikum Carl Gustav Carus Dresden, Institut für Pathologie, Dresden, Deutschland

Einleitung: Die akute myeloische Leukämie (AML) macht ca. $20 \%$ aller Leukämien und somit $4 \%$ aller malignen Erkrankungen im Kindes- und Jugendalter aus. Myelosarkome treten als extramedulläre Manifestation in bis zu $20 \%$ der Patienten mit AML auf. Typische Lokalisationen sind Haut, Orbita, Gingiva und ZNS. Wir berichten von einem Säugling mit ungewöhnlicher Lokalisation und Ausdehnung eines Myelosarkoms als Erstmanifestation einer AML.

Kasuistik: Ein bisher gesunder 7-Monate alter Junge mit seit 2 Tagen zunehmender Zustandsverschlechterung und massiver Tachydyspnoe bei ausgeprägtem rechtsseitigem Pleuraerguss wurde uns unter dem Verdacht auf eine schwere Pleuropneumonie verlegt. Das Kind war afebril, das $\mathrm{CrP}$ mit 14,5 mg/l leicht erhöht, Leukozytenzahl und -differenzierung unauffällig. Nach Aufnahme wurde der Erguss mittels Thoraxsaugdrainage entlastet. Im Pleurapunktat fielen reichlich tumorverdächtige Zellen auf, welche sich durchflusszytometrisch myeloischen Blasten zuordnen ließen. Im Knochenmark fanden sich morphologisch lediglich $8 \%$ blastäre Zellen. Die Thorax-CT zeigte einen vom Mediastinum ausgehenden nach intraspinal ziehenden Tumor mit Verdacht auf ein Lymphom oder Neuroblastom. In der nachfolgenden MRT war die Tumorausdehnung entlang der Pleura mit partieller Infiltration der Thoraxwand, des Mediastinums und per continuitatem durch die Neuroforamina nach extraaxial intraspinal sowie nach intraabdominell entlang der großen Gefäße zu erkennen. Der intraspinale Anteil führte zu einer deutlichen Myelonkompression mit beginnender Parese der unteren Extremitäten. Mittels Tumorbiopsie wurde der Verdacht auf eine extramedulläre AML in dieser seltenen Lokalisation bestätigt. Zunächst erfolgte die Stratifizierung in die intermediäre Risikogruppe gemäß Therapieprotokoll AML-BFM 2012. Zytogenetisch zeigte sich eine $6 \mathrm{q}$-Abberation, welche nicht stratifizierungsrelevant ist. 
Eine Zuordnung zur FAB-Klassifikation konnte nicht getroffen werden. Unter Gabe von Dexamethason zur Prävention eines spinalen Ödems begannen wir die zytoreduktive Vorphase mit Cytarabin als Dauerinfusion über $72 \mathrm{~h}$. Ein Tumorlysesyndrom trat nicht auf. Es kam zu einer raschen klinisch-neurologischen Besserung, so dass eine neurochirurgische Intervention vermieden wurde. Anschließend wurde die Polychemotherapie gemäß dem Therapieprotokoll fortgesetzt. Im Verlauf zeigte sich nach dem 2. Induktionskurs mit $6 \%$ residuellen Blasten keine Remission. Daraufhin erfolgte die Stratifizierung in den High-Risk-Arm mit der Indikation zur Stammzelltransplantation.

Schlussfolgerung: Unser Bericht zeigt, wie komplex im Einzelfall die Diagnostik einer primär extramedullären Manifestation einer AML ist und in Abhängigkeit von der Lokalisation des Myelosarkoms daraus ein onkologischer Notfall resultieren kann. Uns ist kein Einzelfallbericht bekannt, der ein so ausgeprägtes, medio-thorako-spinal gelegenes Myelosarkom als Erstmanifestation einer AML im Säuglingsalter beschreibt.

\section{DGKJ-PO 14}

Hepatitis-assoziierte aplastische Anämie: Bericht über 2 Fälle und systematisches Review der Literatur

\section{Sofia Tsaka', Alexandra Kavvalou², Alexandra Terzi ${ }^{2}$, Rita Beier ${ }^{3}$}

'Universitätsklinikum Essen, Kinderklinik III, Essen, Deutschland; ${ }^{2}$ Universit ätsklinikum Essen, Kinderklinik, Essen, Deutschland; ${ }^{3}$ Universitätsklinikum Essen, Kinderonkologie-KMT, Essen, Deutschland

Hintergrund: Hepatitis-assoziierte aplastische Anämie (HAAA) ist eine seltene Variante der schweren aplastischen Anämie, bei der eine Hepatitis-Episode dem Auftreten einer aplastischen Anämie vorausgeht. Das Knochenmarkversagen ist häufig rasant, schwergradig und in der Regel tödlich, wenn es unbehandelt bleibt, mit Mortalitäten-Raten bis zu $88 \%$. Die beiden wichtigsten aktuellen Therapien für die schwere aplastische Anämie sind die allogene Knochenmarktransplantation (BMT) und die immunsuppressive Therapie (IST) mit Anti-Thymozyten-Globulin (ATG). Ziel dieses Berichts ist, das hohe Potenzial für Morbidität und Mortalität dieser Krankheit herauszustellen.

Fragestellung: Assoziation zwischen Non-A-E-Hepatitis und schwerer aplastischer Anämie.

Material und Methoden: Fallbericht von 2 Patienten und systematisches Review der Literatur in der Pubmed- und Cochrane-Datenbank.

Ergebnisse: Beide Patienten wurden mit IST (immunsuppressive Therapie einschließlich Antithymozytenglobulin, Prednison und Cyclosporin) behandelt und erhielten eine unterstützende Therapie mit G-CSF. Einer von den beiden hat eine partielle Remission erreicht und der andere hat keine Remission erreicht und wird eine allogene Knochenmarktransplantation erhalten.

Diskussion: Hepatitis-assoziierte aplastische Anämie ist ein seltenes, aber gut bekanntes klinisches Syndrom, bei dem eine Episode einer akuten $\mathrm{He}-$ patitis von Zytopenien mit Knochenmarkshypozellularität gefolgt wird. Hepatits, die eine Hepatitis-assoziierte aplastische Anämie (HAAA) vorausgeht ist durch eine Erhöhung der Aminotransferasen und eine Cholestase mit konjugierter Hyperbilirubinämie gekennzeichnet und verläuft in den meisten Fällen gutartig. Die Ätiologie der Hepatitis ist weitgehend unbekannt. Die Rolle von hepatotropen Viren wird weitgehend in Frage gestellt. HAAA entspricht 2 bis $5 \%$ der Fälle schwerer aplastischer Anämie. Knochenmarkversagen tritt 0 bis 3 Monate nach Auftreten einer Hepatitis auf. Klinische Merkmale und neueste Erkenntnisse deuten stark auf eine immunvermittelte Pathophysiologie mit Knochensuppression durch T-Zellen und Leberinfiltration durch aktivierte CD8-Zellen hin. Zusammenfassend ist eine regelmäßige Kontrolle des Blutbilds bei Kindern mit diagnostizierter Non-A-E-Hepatitis wichtig, um eine aplastische Anämie möglichst früh zu erkennen.

\section{Literatur}

1. Weiler-Normann C, Hartl J, Weidemann S, von Pein UM, Fiedler W, Schramm C, Brinkert $F$ (2018) Kröger N3 Acute hepatitis as a prequel to very severe aplastic anemia. Z Gastroenterol 56(1):51-54. https://doi.org/10.1055/s-0043-121737

\section{DGKJ-PO 15}

\section{Jahre alte Patientin mit Langzeitverlauf eines mehrfach-} rezidivierten hepatozellulären Karzinoms

Wiebke Klock', Gerd Steinau' ${ }^{2}$ Sabine Schacht ${ }^{3}$, Till Braunschweig', Angeliki Pappas', Udo Kontny', Lisa Lassay'

'Uniklinik RWTH Aachen, Kinder- und Jugendmedizin, Aachen, Deutschland;'Uniklinik RWTH Aachen, Chirurgie, Aachen, Deutschland;'Uniklinik RWTH Aachen, Radiologie, Aachen, Deutschland; ${ }^{4}$ Uniklinik RWTH Aachen, Pathologie, Aachen, Deutschland

Das hepatozelluläres Karzinom ist eine seltene Erkrankung bei Kindern und Jugendlichen. Therapiestandard ist derzeit eine Kombination aus Platin-haltiger Chemotherapie und dem Tyrosinkinaseninhibitor Sorafenib mit Tumorresektion. Die Heilungschancen betragen $<50 \%$.

Bei bekannter Anorexia nervosa wurde Ende Januar 2011 bei der damals 16 Jahre alten Patientin eine Abdomen-Sonographie aufgrund einer Lipase-Erhöhung durchgeführt. Dabei zeigte sich eine Raumforderung von $2,8 \times 2,4 \times 3,5 \mathrm{~cm}$ in den Lebersegmenten IV, V und VII, die durch eine Hemihepatektomie R0-reseziert wurde und sich histologisch als ein mittelgradig differenziertes hepatozelluläres Karzinom vom fibrolamellären Typ G2 erwies.

Im Staging zeigt sich eine retroperitoneal gelegenene LK-Metastase, die operativ entfernt wird. Die weitere Therapie erfolgt nach Empfehlung der Studiengruppe Lebertumoren mit Chemotherapie (PLADO) und einer Erhaltungstherapie mit Sorafenib. Unter Sorafenib entwickelt die Patientin Nebenwirkungen mit Übelkeit, Bauchschmerzen, Diarrhoen und Akne. Das erste Rezidiv (7/12) manifestiert sich drei Monate nach Ende der Sorafenib-Therapie mit 2 extrahepatischen, intraabdominellen Metastasen. Es erfolgt eine erneute platinhaltige Chemotherapie plus Bevazicumab sowie Metastasenresektion und anschließender Erhaltungstherapie mit Erlotinib $(9 / 12$ bis $8 / 13)$.

Im November 2013 Diagnose eines zweiten Rezidivs mit Peritonealkarzinose und Behandlung mit einem präoperativen und zwei postoperativen Zyklen Oxaliplatin/Gemcitabine und Sorafenib sowie en-bloc subtotaler Peritonektomie, Hysterektomie, Adnektomie und Rektumteilresektion mit HIPEC (Oxaliplatin). Bei drittem Rezidiv im Dezember 2014 in der Appendix vermiformis und am Peritoneum erfolgte eine Resektion aller Tumormanifestationen und eine zweite HIPEC mit Oxaliplatin und Gemcitabin.

Anschließend war die Patientin bis Mai 2016 in Remission. Bei erneutem Rezidiv (4.) mit intraabdominellen Lymphknotenmetastasen und peritonealen Absiedlungen, wurde eine operative Entfernung der Läsionen durchgeführt. Im Anschluss behandelten wir die Patientin off label mit dem PD-1 Checkpoint-Inhibitor Nivolumab. Unter dieser Therapie entwickelte die Patientin eine Steroid- und Infliximab-refraktäre Kolitis Grad 3 bis 4.

Im April 2019 wurde eine Metastase (5. Rezidiv) in Segment III der Leber diagnostiziert und mittels Radiofrequenzablation behandelt. Hinweise auf weitere Metastasen liegen nicht vor. Die Patientin hat weiterhin die Symptome der Colitis. Sie hat während der Erkrankung eine Ausbildung erfolgreich abgeschlossen und führt einen eigenen Haushalt.

Die Besonderheit des Falles besteht in wiederholten Rezidiven eines HCCs mit teilweise mehr als zwei Jahre langen rezidivfreien Intervallen. Der Fall deutet auf die Effektivität bestimmter Behandlungsverfahren wie der HIPEC oder Checkpoint-Inhibition hin, zeigt aber gleichzeitig die Grenzen der Behandlung aufgrund ausgeprägter Therapie-assoziierter Nebenwirkungen auf. 


\section{DGKJ-PO 16}

Kongenitaler Protein C Mangel: Inadäquate Therapie der Erstmanifestation (Thrombose) führt zu schwerwiegenden Langzeitfolgen für ein 14jähriges Mädchen

\section{Madlen Reschke', Wolfgang Eberl ${ }^{2}$, Hans-Jörg Hertfelder ${ }^{3}$}

'Universitätsklinikum Essen, Klinik für Kinder und Jugendmedizin, HämatoOnkologie, Essen, Deutschland:'25tädtisches Klinikum Braunschweig, Kinderonkologie-Hämostaseologie, Braunschweig, Deutschland; ${ }^{3}$ Universitätsklinikum Bonn, Transfusionsmedizin, Bonn, Deutschland

Ein schwerer Protein C Mangel ist ein signifikanter Risikofaktor für die Entstehung schwerwiegender, thromboembolischer Ereignisse. Mangelnde Kentniss dieser Erkrankung führte zu schwerwiegenden, lebenslangen Konsequenzen für unsere 14jährige Patientin.

Erstvorstellung eines 14 Jahre alten Mädchens mit spontaner, erster tiefer Venenthrombose im rechten Bein. Bekannter Protein C Mangel mit aktuell 7 \% Restaktivität.

Behandlung: Unfraktioniertes Heparin iv. Klinische Verschlechterung binnen 10 Tagen (Kompartmentsyndrom, Lungenembolie bds.). Die Patientin entwickelte durch das gangränöse Bein eine hämodynamisch relevante Sepsis. Es erfolgte die fraktionelle Beinamputation im rechten Hüftgelenk. Weitere Komplikationen im Rahmen der Intensivbehandlung: Tracheostoma, massive Thrombose im linken Bein, arterielle Verschlüsse im linken Arm, Aggression, Angst und Depression. Es wurden mehrere venöse und arterielle Thrombektomien durchgeführt. Die Kinder- und Jugendpsychiartrie wurde hinzugezogen.

10 Tage nach der Amputation wurde Kontakt zur pädiatrischen Hämostaseologie aufgenommen. Die Familienanamnese war deutlich positiv für klinische manifeste Thrombophilie, Protein C Mangel und Faktor V Leiden Mutation.

Die Behandlung wurde auf Protein $\mathrm{C}$ iv und Enoxaparin sc umgestellt. Seither keine weiteren, relevanten thrombotischen Ereignisse. Im Verlauf erfolgte die Umstellung auf Protein C sc für 5 Monate und Apixaban po statt Enoxaparin sc.

Nach 5 Monaten im MRT komplette Rekanalisierung der Thrombosen im linken Bein bei persistierenden arteriellen narbigen Veränderungen im linken Arm nach rezidivierenden Thrombektomien.

Aktuelle Dauertherapie (14 Monate nach Erstdiagnose): Apixaban po und ASS po sowie andauernde Physiotherapie.

Zusammenfassung: Die Therapieumstellung auf Protein C und Enoxaparin unterbrach schnell und effektiv das wiederholte Auftreten thromboembolischer Ereignisse in der akuten Phase und führte zur Rekanalisierung ausgeprägter tiefer Venenthrombosen. Spontane Thrombosen im Kindesalter sind eine Rarität. Besonders bei Kindern ist die Familienanamnese ausgesprochen wichtig! Es sollte nach dem auslösenden Faktor gesucht werden und dieser sollte, wenn möglich auch behandelt werden. Kritisch kranke Kinder sollten immer auch von Pädiatern betreut werden.

\section{DGKJ-PO 17 \\ Diffus großzelliges B-Zelllymphom bei einem 19 Jahre alten Patienten mit Morbus Crohn}

Annamaria Florian', Udo Kontny², Angeliki Pappa33, Sabine Maria Schacht', Norbert Wagner ${ }^{5}$, Lisa Lassay ${ }^{2}$, Till Braunschweig ${ }^{6}$

'Aachen, Deutschland; ${ }^{2}$ Uniklinik RWTH Aachen, Pädiatrische HämatoOnkologie, Aachen, Deutschland; ${ }^{3}$ Uniklinik RWTH Aachen, pädiatrische Endokrinologie und Gastroenterologie, Aachen, Deutschland; ${ }^{4}$ Uniklinik RWTH Aachen, Radiologie, Aachen, Deutschland;'Uniklinik RWTH Aachen, Kinderklinik pädiatrische Gastroenterologie und Rheumatologie" Aachen, Deutschland; ' $U$ niklinik RWTH Aachen, Pathologie, Aachen, Deutschland

Patienten mit chronisch-entzündlichen Darmerkrankungen weisen unter immunsuppressiver Therapie ein dreifach erhöhtes Risiko für die Entwicklung von Non-Hodgkin Lymphomen auf.

Fallbericht: Bei dem Patienten wurde im Alter von 10 Jahren nach einer 6-monatigen Vorgeschichte von Bauchschmerzen, blutigen Stühlen, Perianalabszessen und Gewichtsabnahme die Diagnose eines M. Crohn gestellt mit primärem Befall des Kolons mit diskontinuierlicher Entzündung und Aphthen mit Beteiligung der Bauhinschen Klappe und schweren Veränderungen im Bereich des Sigmas. Die Behandlung erfolgte zunächst mit Steroid und Azathioprin. Bei steroidabhängigem Verlauf wurde Infliximab eingesetzt (2010) und Azathioprin abgesetzt, bei Therapieversagen wurde seit 2013 Adalimumab in 14tägigen Intervallen in Standarddosis gegeben. Im Alter von 19 Jahren entwickelte der Patient zunehmende Schmerzen im Bereich des linken Oberschenkels und der Hüfte. Im Zeitraum von 3 Monaten habe er zudem $4 \mathrm{~kg}$ verloren. In der auswärtig durchgeführten RöntgenUntersuchung des Knies sei eine unklare Knochenstruktur aufgefallen. Bei sonographisch festgestellter Synovia-Verdickung im Knie- und im Hüftgelenk, ergab sich die Indiaktion für eine MRT Untersuchung. Hierbei ergab sich ein auffälliges Knochenmarksignal, ohne Hinweise auf eine Destruktion der Kortikalis oder Weichteilinfiltration. Bei Verdacht auf einen malignen Prozess wurde die Knochenläsion biopsiert und die Diagnose eines diffus großzelligen B-Zelllymphoms vom ABC-Subtyp gestellt. Im Staging mittels PET-/CT zeigten sich 2 weitere Herde, ein ossärer Herd in BWK 11 und eine Raumforderung mit Haupttumorlast in den Segmenten VI und VII und geringem Einwachsen in die Segmente V und VIII der Leber.Damit lag ein Stadium II nach der St. Jude's Klassifikation vor. Eine Therapie wurde nach dem BFM-NHL 2012 Register begonnen. Bei nicht erhöhter LDH erfolgte eine Zuordnung in die Therapiegruppe II, bestehend aus einer Vorphase und 4 Chemotherapieblöcken. Bei starker CD20-Expression der Lymphomzellen erfolgte zusätzlich die Gabe von 7 Dosen Rituximab. Der Patient hat die Chemo gut vertragen, jedoch keine Zeichen der Lyse gezeigt. Auch die sonographischen Kontrollen des Leberrundherds zeigten nur wenig Größenregredienz. Zunächst wurd auf eine Crohn-spezifische Therapie verzichtet, da angenommen wurde, dass die anti-entzündliche Wirkung der NHL Therapie ausreiche. In einer durchgeführten KontrollEndoskopie zeigte sich eine ausgeprägte Stenose (die seit Beginn der Chemotherapie bekannt war) im Sigmoid mit mittlerer Entzündungsaktivität, daher wurde eine Therapie mit Vedolizumab begonnen.

Die Assoziation eines NHL mit chron. entzündlichen Darmerkrankungen ist bekannt. In der Regel sind dies periphere T-Zell-Lymphome, vor allem hepatolienale PTCL. Als Risikofaktor für die Entwicklung eines NHL Lymphoms ist die Therapie mit Purinanaloga beschrieben, ob TNF-Antagonisten auch einen Risikofaktor darstellen könnten, wird kontrovers diskutiert.

\section{DGKJ-PO 18}

\section{Hämatochromatose Typ 4}

Jacob Eschweiler', Lisa Lassay², Ingo Kurth' ${ }^{3}$, Norbert Wagner ${ }^{1}$

'Uniklinik RWTH Aachen, Kinder- und Jugendmedizin, Aachen,

Deutschland; ${ }^{2}$ Uniklinik RWTH Aachen, Kinder- und Jugendmedizin, Sektion pädiatrische Hämatologie und Onkologie, Aachen, Deutschland; ${ }^{3}$ Uniklinik RWTH Aachen, Institut für Humangenetik, Aachen, Deutschland

Ein 12-jähriger Junge mit Hämatochromatose vom sehr seltenen Typ 4. Es handelt sich dabei um eine Mutation im SLC40A1-Mutation (p.G494D) aufChromosom 2q32.2, welches für den Eisentransporter Ferroportin kodiert. Zu dieser Mutation besteht bisher noch keine Literatur, sodass wir es als sinnvoll erachten, den dazugehörigen klinischen Verlauf zu publizieren. Auffällig wurde er durch ein erhöhtes Ferritin von $1290 \mathrm{ng} / \mathrm{ml}$ (Ref. 25-112 ng/ml) im Rahmen einer Routineblutentnahme. Die Transferrinsättigung, der lösliche Tranferrinrezeptor, das Serumeisen und das Coeruloplasmin waren unauffällig. Die klinische und augenärztliche Untersuchung waren ebenfalls unauffällig. Die Familienanamnese ist für Hämatochromatose ist unauffällig. Bei der Mutter war ein leicht erhöhtes Ferritin von 193 ng/ml (Ref. 15-150 ng/ml) auffällig. Es konnte keine typische Mutation HFE-Gen nachgewiesen werden, sodass erst in erweiterter genetischer Diagnostik die oben genannte seltene genetische $\mathrm{Mu}$ tation nachgewiesen wurde. Im MRT Abdomen konnte eine beginnende Leberhämosiderose festgestellt werden. Weitere Familienmitglieder scheinen nicht betroffen zu sein.

Weitere Untersuchungen müssen noch durchgeführt werden, um die Pathogenität dieser Mutation genauer darstellen zu können. 


\section{DGKJ-PO 19}

\section{Häufigkeit und Art der stationären Behandlungen der} Fibrodysplasia ossificans progressiva (FOP) von 2005 bis 2017

Steffen Wahler', Clemens Stockklausner ${ }^{2}$, Alfred Mueller ${ }^{3}$

'keine, keine, Hamburg, Deutschland;'Klinikum Garmisch-Partenkirchen, Kinderheilkunde, Garmisch-Partenkirchen, Deutschland; ${ }^{3}$ Analytic Services $\mathrm{GmbH}$, Demographie, München, Deutschland

Einführung: Die Fibrodysplasia ossificans progressiva (FOP) beschreibt eine sehr seltene, krankhafte, chronisch fortschreitende Verknöcherung des Binde- und Stützgewebes. Ursächlich ist eine Mutation in der Steuerung der Skelettbildung. In der Folge entwickeln Fibrozyten bei Wundheilung Knochen statt Narbengewebe, was bei Verletzungen zum Versteifen des Körpers durch Verknöcherung führt. Derzeit bestehen nur palliative Therapieoptionen.

Für Deutschland gibt es nur wenige epidemiologische Daten. Kürzlich wurde für Frankreich eine qualifizierte Schätzung ermittelt, der eine Prävalenz von 112 Patienten in Deutschland entsprechen würde.

FOP hat einen spezifischen ICD-Code (M61.1). Hier sollen die stationären Aufenthalte von Patienten, die als FOP diagnostiziert wurden, über 11 Jahre untersucht werden und mit den Ergebnissen aus Frankreich in Beziehung gesetzt werden.

Methoden: Daten der stationären Versorgung des statistischen Bundesamtes 2005-2015, des Instituts für das Entgeltsystem im Krankenhaus (InEK) und die Qualitätsberichte für das Jahr 2015 wurden auf FOP-Kodierungen untersucht und ausgewertet. Verwendet wurden Microsoft-Excel und Microsoft-Access (Version 2016).

Ergebnisse: Im Jahr 2015 wurden 216 stationäre Fälle mit FOP kodiert, 108 (2005: 90) als Hauptdiagnose und 108 als Nebendiagnose (2005: 136) Die Fallzahl schwankte mit einem Minimum 2006 und 2014 mit 200 und einem Maximum 2010 mit über 300 Fällen. 39 \% der Behandlungen waren 2015 in Kinderabteilungen, was an der Versorgung in Garmisch-Partenkirchen liegt. $16 \%$ der Fälle wurden in universitären Einrichtungen behandelt. $45 \%$ der Fälle waren in orthopädisch-chirurgischen Fachabteilungen. In $44 \%$ waren Becken und Oberschenkel betroffen, in $13 \%$ multiple Lokationen. Eingriffe an der Hand waren mit mittleren Alter von 18 bei den jüngsten Patienten. Das Durchschnittsalter lag bei 35,9 Jahren, Median bei 26 Jahren. Die Geschlechtsverteilung war insgesamt stets etwa hälftig. Nach Bundesland des Wohnort der Patienten sind die Fälle etwa gleich verteilt, mit geringen Ausreißern nach oben (Bremen) und unten (Sachsen). Patienten werden möglicherweise mehrfach jährliche stationär behandelt, daher kann Fallzahl nicht mit Patientenzahl gleichgesetzt werden. Fehlkodierungen können nicht überprüft werden.

Schlussfolgerungen: FOP ist auch in Deutschland eine sehr seltene Erkrankung. Aus den Krankenhausfalldaten kann keine exakte Prävalenz abgeleitet werden. Genauere Angaben könnte weitere Forschung und die systematische Beteiligung an einem Register liefern. Die Ergebnisse entsprechen den französischen Daten nach Alter und Geschlechtsverteilung. Hinzu kommen Informationen zur Lokation der Erkrankung und den behandelnden Abteilungen. Im Unterschied zu ähnlich seltenen schweren Erkrankungen erfolgt nur eine Minderheit der Versorgung in universitären Zentren.

\section{DGKJ-PO 20}

\section{Granulomatosis with polyangiitis-a rare vasculitis in children. Presentation with a puzzle of unspecific initial symptoms}

Michèle Roth', Tatjana Welzel', Christoph Rudin ${ }^{3}$, Thomas Daikeler', Andreas Wörner?

'University Children's Hospital Basel (UKBB), University of Basel, Switzerland, Pediatric Rheumatology, Basel, Switzerland;'2University Children's Hospital Basel (UKBB), University of Basel, Switzerland, Pediatric Pharmacology, Pediatric Rheumatology, Basel, Switzerland; ${ }^{2}$ University Children's Hospital Basel (UKBB), University of Basel, Switzerland, Pediatric Nephrology, Basel, Switzerland; ${ }^{4}$ University Hospital of Basel, University of Basel, Switzerland, Rheumatology, Basel, Switzerland
Introduction: Granulomatosis with polyangiitis $(\mathrm{GwP})$ is a rare vasculitis in children affecting small and medium sized vessels with involvement of different organ systems. Disease onset can be unspecific with progression to a life-threatening condition.

Methods and objectives as case report: A 12 year old boy presented with pneumonia. Amoxicillin/clavulanic acid and clarithromycin were started. He was sent to hospital due to persisting fever and worsening respiratory symptoms. The personal history included chronic rhinitis and recurrent otitis, treated several times unsuccessfully with antibiotics. A nasal polypectomy was performed one year ago. Family history was unremarkable. The initial workup revealed anaemia, mild inflammation and basal infiltrates in the chest X-ray, suggesting severe pneumonia. Antibiotics were changed to ceftriaxone. With progression of respiratory distress, ongoing fever, development of purpura-like skin lesions, increased inflammatory markers with hyperferritinemia, negative blood cultures, negative nasopharyngeal swap and presence of urinary glomerular erythrocytes vasculitis was suspected. Due to rapid deterioration with respiratory failure the patient was transferred to the intensive care unit. CT scan showed bipulmonal peribronchovascular infiltrates, identified as alveolar haemorrhage by bronchoscopy. Highly increased PR3-ANCA indicated the diagnosis of GwP, supported by skin and kidney biopsy. The retrospective analysis of the previous conducted nasal polypectomy showed chronic granulomatous inflammation.

High dose steroids and a single dose of cyclophosphamide were given. Plasmapheresis was conducted for 5 days with decrease of PR3-ANCA. Further therapy included rituximab given weekly for one month, then every 6 month. Prednisone therapy was continuously tapered to $4 \mathrm{mg}$ per day after 6 months.

Results: The patient fulfilled 5/6 EULAR/PRINTO/PRES and 5/5 ACR criteria for GwP. Recovery was excellent with complete clinical remission 9 months after initial presentation. In the last follow up no clinical complaints were present. Pulmonary follow up revealed mild pulmonary obstruction and occlusive nasal breathing. Arthritis, skin lesions, arterial hypertension, proteinuria or haematuria were absent. Under rituximab therapy laboratory showed normalization of PR3-ANCA while leaving IgG levels within the normal range.

This outcome is comparable with study outcomes after 12 months in children with GwP, who showed persisting renal (36\%), upper airway (21\%) and pulmonary (16\%) symptoms.

Conclusion: GwP can show unspecific initial symptoms (chronic rhinitis, recurrent otitis media) with life-threatening progression. Immediate, aggressive and interdisciplinary therapy of this rare severe vasculitis is mandatory. Until now, no standardized therapy guidelines are available for pediatric age.

\section{Infektologie/Epidemiologie}

\section{DGKJ-PO 21}

\section{Jungen sterben häufiger an nekrotisierender Enterokolitis als Mädchen - Ergebnisse der deutschlandweiten Krankenhausstatistik von 2000 bis 2017}

Arne Schröder 1 , Oliver Muensterer ${ }^{2}$, Christina Oetzmann von Sochaczewski ${ }^{3}$ ${ }^{1}$ Krankenhaus Düren, Anästhesie, Düren, Deutschland; ${ }^{2}$ Universitätsme dizin Mainz, Kinderchirurgie, Mainz, Deutschland; ${ }^{3}$ Universitätsmedizin, Johannes-Gutenberg-Universität Mainz, Kinderchirurgie, Mainz, Deutschland

Fragestellung: Vereinzelt wurde gezeigt, dass Jungen ein höheres Risiko haben eine nekrotisierende Enterokolitis zu entwickeln; weit häufiger jedoch wurde diese Hypothese verworfen. Allerdings ist eine geringe Effektstärke naheliegend, so dass es einer hinreichend großen Fallzahl bedarf um diese aufzudecken, welche von den üblichen monozentrischen Studien kaum erreicht werden kann. Wir nutzten daher die bundesweiten 
statistischen Daten um die Hypothese zu prüfen, ob Jungen häufiger an nekrotisierender Enterokolitis sterben als Mädchen.

Material und Methode: Wir werteten die tiefgegliederten Diagnosedaten der deutschen Krankenhausstatistik für die Jahre 2000 bis 2017 für die Fachabteilungshauptdiagnose P77-nekrotisierende Enterokolitis-getrennt nach Geschlechtern und Überleben aus. Um einen Zusammenhang zwischen Geschlecht und Versterben respektive dem Erkrankungsrisiko zu untersuchen verwendeten wir den exakten Test nach Fisher mit einem einseitigen Test auf höheres Risiko für Jungen.

Ergebnisse: In den Jahren 2000 bis 2017 hatten 3119 Neugeborene die Fachabteilunghauptdiagnose nekrotisierende Enterokolitis, davon 1769 $(0,27 \%)$ von 6.541 .428 männlichen und $1350(0,22 \%$ o) von 6.208 .422 weiblichen Lebendgeborenen. Als Todesursache wurde die nekrotisierende Enterokolitis für 173 Jungen (9,8\%; 95\% CI: 8,4-11,2\%) und 106 Mädchen (7,9\%; 95\% CI: 6,5-9,4\%) angegeben. Das männliche Geschlecht ist ein Risikofaktor fürs Versterben im Rahmen einer nekrotisierenden Enterokolitis mit einem relativen Risiko von 1,246 $(\mathrm{P}=0,035)$. Gleichermaßen ist das Erkrankungsrisiko für Jungen bezogen auf die Lebendgeburten relativ um 1,24 erhöht $(\mathrm{P}<0,001)$

Diskussion: Ein wesentlicher Vorteil der Krankenhausstatistik ist der große Umfang der Studienpopulation mit 3119 Kindern in 18 Jahren als auch die parallel verfügbaren Zahlen derselben Population zur Todesursache. Die Zuordnung zur Diagnose der nekrotisierenden Enterokolitis erfolgt anhand der Fachabteilungshauptdiagnose, womit zwar sichergestellt wird, dass dies die im Vordergrund stehende Diagnose ist, allerdings auch eine wesentliche Schwäche der Population beschrieben wird. Es ist naheliegend, dass mildere Verlaufsformen der nekrotisierenden Enterokolitis nicht durch diese Statistik erfasst werden, da sie nicht das Kriterium der Hauptdiagnose erfüllen, wenn zeitgleich eine andere Diagnose im Vordergrund steht, die den Krankenhausaufenthalt noch mehr veranlasst hat respektive mehr Ressourcen verbraucht hat. Auch ermöglicht die Krankenhausstatistik keine Adjustierung für mögliche verzerrende Faktoren wie Geburtsgewicht, Schwangerschaftsdauer oder komplizierende Nebendiagnosen. Diese Faktoren stehen jedoch im umfangreichen Datensatz der Neonatalerhebung zur Verfügung.

Schlussfolgerung: Jungen sterben häufiger als Mädchen an nekrotisierender Enterokolitis; in den Daten der Krankenhausstatistik. Der weit umfangreichere und detaillierte Datensatz der Neonatalerhebung wäre geeignet diese Assoziation zu bestätigen.

\section{DGKJ-PO 22}

\section{Schlafauffälligkeiten bei Kindern und Jugendlichen häufig}

Christiane Lewien' ', Christof Meigen 1', Wieland Kiess'2, Tanja Poulain ${ }^{2}$

'Universität Leipzig, Leipziger Forschungszentrum für

Zivilisationserkrankungen (LIFE Child), Leipzig, Deutschland;'universität Leipzig, Leipziger Forschungszentrum für Zivilisationserkrankungen (LIFE Child), Department für Frauen- und Kindermedizin, Klinik und Poliklinik für Kinder- und Jugendmedizin, Center for Pediatric Research (CPL), Leipzig, Deutschland

Hintergrund: Gemeinhin ist bekannt, dass zu wenig oder beeinträchtigter Schlaf vielfältige negative Konsequenzen hat. Es ist demnach alarmierend, dass Schlafstörungen im Kindes- und Jugendalter in den letzten Jahren zugenommen haben.

Fragestellung: Worin liegen die Schlafprobleme und wie sind sie verteilt? Zur Beantwortung dieser Fragen haben wir das Schlafverhalten von Kindern und Jugendlichen unter Berücksichtigung von Alter und Geschlecht und des sozialen Hintergrunds untersucht.

Material und Methoden: Im Rahmen der epidemiologischen LIFE ChildStudie in Leipzig wurden 1902 4-17jährige Kinder und Jugendliche zwischen 2012 und 2015 zu Schlafproblemen befragt. Von 855 4-9 Jährigen beantworteten ihre Eltern den Children's Sleep Habits Questionnaire (CSHQ) und 1047 10-17 Jährige gaben Selbstauskunft mithilfe des Sleep Self Reports (SSR). Bei der Auswertung galt ein Ergebnis in dem Gesamtscore für Schlafprobleme über einem Stanine-Wert von 8 als auffällig. Mit multipler linearer Regressionsanalyse wurden Zusammenhänge zwischen
Schlafproblemen und dem Alter und Geschlecht der Kinder sowie der sozialen Stellung der Familie untersucht.

Ergebnisse: Schlafauffälligkeiten wurden bei $22,6 \%$ der 4-9jährigen und $20,0 \%$ der 10-17jährigen Kinder und Jugendlichen festgestellt. Im Jugendalter war die Prävalenz von auffälligem Schlafverhalten bei Mädchen höher als bei Jungen. Unter den 4-9 Jährigen hatten jüngere Kinder mehr Angst vor dem Schlafen, leisteten mehr Widerstand beim Zubettgehen, litten häufiger unter Einschlafverzögerung, Parasomnien und nächtlichem Aufwachen als ältere Kinder. In beiden Altersgruppen (4-9 und 10-17 Jährige) litten ältere Kinder und Jugendliche häufiger an Tagesschläfrigkeit als jüngere. Zusätzlich zeigten die Analysen bei den 10-17 Jährigen, dass Kinder aus niedrigeren Sozialschichten mehr Schlafprobleme angaben als Kinder aus höheren Sozialschichten.

Diskussion: Der SSR und der CSHQ bieten als Screeningfragebögen für Schlafprobleme zwar weitgehend die Möglichkeit, auffällige Ergebnisse zu bewerten. Jedoch wären weitere Grenzwerte für die Subskalen wünschenswert, um individuelle Problemfelder identifzieren zu können.

Schlussfolgerung: Die Analysen zeigen, dass Schlafprobleme weit verbreitet sind und ungefähr jedes 5. Kind an Schlafproblemen leidet. Besorgniserregend ist zudem, dass Jugendliche aus sozial schwächeren Familien einem erhöhten Risiko für Schlafprobleme ausgesetzt sind.

\section{DGKJ-PO 23}

\section{Sociodemographic factors associated with health literacy in a large sample of mothers of newborn children: cross-sectional findings from a birth cohort (KUNO Kids health study)}

Susanne Brandstetter', Josefine Atzendorf', Birgit Seelbach-Göbel', Michael Melter', Michael Kabesch', Christian Apfelbacher ${ }^{3}$

'Klinik und Poliklinik für Kinder- und Jugendmedizin der Universität Regensburg (KUNO-Kliniken), Regensburg, Germany; ${ }^{2}$ Klinik für Frauenheilkunde und Geburtshilfe der Universität Regensburg, Klinik St. Hedwig, Regensburg, Germany; ${ }^{3}$ Universität Regensburg, Institut für Epidemiologie und Präventivmedizin, Regensburg, Germany

Background: Health literacy can be defined as a person's capabilities to make sound decisions regarding health and determinants of health. It is considered an important public health goal and of particular relevance when people are starting a family. However, health literacy of new mothers has not been studied so far in Germany.

Methods: Health literacy and various sociodemographic variables were assessed among 2182 mothers of newborns who take part in an ongoing birth cohort study (KUNO-Kids health study). Health literacy was measured by the HLS-EU health care scale (Sørensen, 2013) from which an index (range 0-50) was derived with higher values indicating higher health literacy. Sociodemographic variables which were associated with health literacy in univariable linear regression analyses were included in a multivariable regression model.

Results: Almost $40 \%$ of mothers had a limited health literacy level. The mean health literacy index was $35.5(\mathrm{SD}=2.2)$. In multivariable regression, higher education was associated with higher health literacy $(\beta=0.12$, $p<0.001)$ and giving birth for the first time was associated with lower health literacy $(\beta=-0.08, p=0.001)$, respectively.

Discussion: Albeit the mean level of health literacy was high, there was a substantial amount of mothers experiencing problems in dealing with and navigating through the health care system-this applied above all to firsttime mothers and mothers with low education.

Conclusion: These findings are important for the design of paediatric health services. Health care providers should acknowledge that an adaption of professional practices is necessary since many parents have difficulties navigating through the health care system as it is currently designed. 


\section{DGKJ-PO 24}

\section{Umzug einer Kinderklinik: Ändern sich Resistenzen?}

Rudi Ascherl', Annika Schönfeld ${ }^{2}$, Norman Lippmann ${ }^{3}$, Wieland Kiess ${ }^{2}$ 'Universitätsklinikum Leipzig, Klinik und Poliklinik für Kinder- und Jugendmedizin, Leipzig, Deutschland;'2Universitätsklinikum Leipzig AöR, Klinik- und Poliklinik für Kinder- und Jugendmedizin, Leipzig, Deutschland; ${ }^{3}$ Universitätsklinikum Leipzig AöR, Institut für medizinische Mikrobiologie, Leipzig, Deutschland

Obwohl Antiinfektiva-Resistenzen als räumliches Phänomen begriffen werden, ist der Einfluss örtlicher Faktoren kaum untersucht. Dies gilt auch für den Umzug medizinischer Einrichtungen. Wir haben die Minimalen Hemmkonzentrationen (MHK) aller aufgelaufenen Isolate aus Blutkulturen, Sputum und Urinproben vor und nach dem Umzug unserer Universitätskinderklinik im Jahr 2007 verglichen. Die Gesamtzahl an Untersuchungen unterschied sich nicht signifikant, wohl aber stiegen der Anteil positiver Befunde und die Summe der bestimmten MHKs. Bestimmte Erreger (A. baumanii, E. faecalis, Klebsiella spp. und P. mirabilis) konnten häufiger nach dem Umzug isoliert werden. Die meisten Unterschiede fanden sich bei E. faecium (Anstiege bei 8 Antiinfektiva, Abfälle nur bei Linezolid und Gentamicin). Bei Imipenem und Aminopenicillinen stiegen die MHK regelhaft nach dem Umzug an; ein gegenläufiger Trend fand sich bei Gentamicin und Cotrimoxazol. Viele der Faktoren, die sich durch den Umzug geändert haben, können wir nicht getrennt aufschlüsseln oder deren Einfluss bestimmen. Die Analyse der MHKs ermöglicht uns aber den Blick auf subtile Veränderungen bei einzelnen Wirkstoffen, die sich den üblicherweise betrachteten starren Kategorien „sensibel“, „intermediär“ und „resistent" entziehen würden.

\section{DGKJ-PO 25}

\section{Asplenie - ein angeborener Immundefekt gegen bekapselte Erreger. Fallpräsentation einer fulminanten Pneumokokkensepsis bei Heterotaxiesyndrom}

\section{Barbara Fiedler', Oliver Schwartz', Ingeborg van den Heuvel', Heymut Omran}

'Klinik für Kinder- und Jugendmedizin, Universitätsklinikum Münster, Allgemeine Pädiatrie, Neuropädiatrie, Münster, Deutschland; ${ }^{2}$ Klinik für Kinder- und Jugendmedizin, Universitätsklinikum Münster, Allgemeine Pädiatrie, Pädiatrische Intensivmedizin, Münster, Deutschland

Hintergrund: Eine angeborene Asplenie/Hyposplenie ist selten und tritt oft im Rahmen eines Heterotaxiesyndroms auf. Das Heterotaxiesyndrom ist eine sehr heterogene Krankheitsgruppe mit einer Inzidenz von 1/10.000 bis 1/40.000. Veränderungen der Milz mit Asplenie/Hyposplenie aber auch Polysplenie treten neben den Herz- und Gefäßfehlbildungen und den Viszeralorganisomerien oft auf. Häufigere Ursachen für eine Asplenie/Hyposplenie im Kindesalter sind erworben, wie die Splenektomie und die funktionellen Asplenie bei Sichelzellanämie durch Mikroinfarkte. Eine isolierte, angeborene Asplenie ist extrem selten.

Bei Funktionsverlust der Milz ist die Immunabwehr gegen bekapselte Erreger wie Strep. pneumoniae, N. meningitidis und H. influenzae Typ b deutlich eingeschränkt. Das Risiko für eine fulminante Sepsis durch bekapselte Erreger ist 200-fach erhöht, die Letalität hoch.

Fallpräsentation: Wir berichten von einem 13 Monate alten Jungen mit Heterotaxiesyndrom (inkompletter Situs inversus abdominalis ohne Herzfehler) und V.a. Asplenie, der an einer fulminanten Pneumokokkensepsis erkrankte. Postpartal wurden in den Blutausstrichen keine HowellJolly-Körperchen gefunden, sonographisch konnte keine Milz dargestellt werden. Er wurde vollständig nach STIKO-Empfehlungen geimpft, inkl. Pneumokokkenimpfung, eine Penicillinprophylaxe bekam er nicht. Die Initialsymptome mit Erbrechen ließen an eine Gastroenteritis denken, so dass nicht unmittelbar mit einer antibiotischen Therapie begonnen wurde. Innerhalb von $24 \mathrm{~h}$ kam es zum multiplen Organversagen mit schwerer Meningoenzephalitis und multiplen Hirnabszessen. Unter intensivmedizinischer Behandlung inklusive ECMO-Therapie über 10 Tage konnte eine stabile Kreislaufsituation erzielt und der Patient extubiert werden. Mit schwersten neurologischen Sequelae bei ausgedehnten Kolliquationsnekrosen der Hirnabszesse erfolgte eine primär palliative Versorgung des $\mathrm{Pa}$ tienten. 7 Monate nach dem Akutereignis verstarb der Patient zu Hause. Diskussion: Gerade die angeborene Asplenie/Hyposplenie ist - wenn auch selten - eine wichtige und dringlich zu stellende Diagnose in der Kinderheilkunde. Bei Heterotaxiesyndromen ist eine Diagnostik auf die Milzfunktion/-anlage essenziell. Labordiagnostisch sind Howell-JollyKörperchen wegweisend, ihr Fehlen schließt eine Asplenie/Hyposplenie aber nicht aus. Patienten mit einer Asplenie/Hyposplenie sind durch bekapselte Erreger lebensbedrohlich gefährdet, die Sensibilisierung auf dieses Thema und das Ergreifen von Vorsichtsmaßnahmen senken dieses Risiko.

Schlußfolgerung: Bei fehlender Milzfunktion sind folgende Maßnahmen dringend indiziert: Aufklärung des Patienten/der Familie mit Ausstellung eines Notfallausweises, Impfungen gegen bekapselte Erreger, Antibiotikaprophylaxe (Zeitrahmen nach Ursache und Alter), sofortige Antibiotikatherapie bei ersten Infektionszeichen (Stand-by-Antibiose zu Hause!).

\section{DGKJ-PO 26}

\section{Ecthyma gangraenosum als Folge einer Pseudomonas-Sepsis bei einem 1,5jährigen Jungen nach Baden im Whirlpool}

Pauline Schumacher', Florian Schneider ${ }^{2}$, Katharina Knop' ${ }^{1}$, Thomas Frank ${ }^{3}$, Johannes Faust ${ }^{1}$, Michael Böswald ${ }^{1}$

${ }^{1}$ St. Franziskus Hospital Münster, Allgemeine Kinder- und Jugendmedizin, Münster, Deutschland;'² St. Franziskus Hospital, Klinik für Pädiatrie und Neonatologie, Münster, Deutschland; ${ }^{3}$ St. Franziskus Hospital Münster, Neonatologie und Pädiatrische Intensivmedizin/Kinderkardiologie, Münster, Deutschland

Einführung: Die Whirlpoolanamnese ist bei Sepsis im Kindesalter ein wichtiger Hinweis auf eine Infektion mit Pseudomonaden. Das Auftreten perianaler Hautveränderungen, bis hin zu tiefen Nekrosen, erfordert eine unmittelbare Therapieeinleitung, da eine Sepsis mit deutlich erhöhter Mortalität einhergeht.

Fallbeschreibung: 1,5jähriger Patient in reduziertem Allgemeinzustand mit seit 1 Woche bestehendem Fieber bis $40{ }^{\circ} \mathrm{C}$, Erbrechen, Durchfall und Nahrungsverweigerung. Bei Aufnahme präsentierte sich der Patient bewusstseinsklar, mit blassem Hautkolorit, reduziertem Hautturgor, Tachykardie (140/min), Blutdruck (112/50 mmHg) und reger Darmperistaltik. Perianal rosettenartig angeordnete, livid-rötliche Läsionen, die bei Berührung schmerzten. Aufnahmelabor: CrP $154 \mathrm{mg} / \mathrm{dl}$, Serumnatrium 128 mmol/l, Panzytopenie (Hb 9,1 g/l, Leukozyten 2,3/nl, Thrombozyten 79/nl) und alterierte Gerinnung (D-Dimere 2,5 mg/l, Fibrinogen $397 \mathrm{mg} /$ dl). Blut-, Urin-, Stuhlkultur und Analabstrich wurden abgenommen. In der Blutkultur Pseudomonas aerigunosa nachgewiesen. Therapie: Bei akuter Dehydratation und Sepsisverdacht mit DIC Volumenbolus mit $\mathrm{NaCl}$ $0,9 \%$, balancierte Elektrolyt-Glukoselösung zum Erhalt, Cefotaxim antibakteriell initial, nach Pseudomonasnachweis in Blutkultur auf Ceftazidim umgestellt, niedermolekulares Heparin (Clexane $1 \mathrm{mg} / \mathrm{kg}$ alle $12 \mathrm{~h}$ ). Bei Thrombozytopenie (min. 12/nl) 1 Thrombozytenkonzentrat und 2 Gefrierplasmakonserven. Analgetisch erhielt der Patient Piritramideinzelgaben und Metamizol-Dauerinfusion. Die perianalen Läsionen wurden mit $\mathrm{NaCl}$ 0,9 \% gespült, mit Lavanidgel und sterilen Kompressen versorgt Verlauf: Patient konnte Klinik nach 14 Tagen deutlich gebessert verlassen. Die primären Nekrosen hinterließen tiefe Ulzerationen. Die Darmentleerungsfunktion und der Sphinkter externus waren von der Nekrose nicht betroffen.

Schlussfolgerung: Klinisches Bild einer Sepsis, die Whirlpoolanamnese und der Nachweis von Pseudomonas aeruginosa in der Blutkultur führen bei den beschriebenen Hautveränderungen zur Diagnose Ecthyma gangraenosum. Diese seltene, schwerwiegende Infektion mit Verschluss kleiner Gefäße und nachfolgender Nekrose ist häufig eine Komplikation invasiver Pseudomonas-Infektionen. Prädilektionsstellen sind die Anogenital- und Axillarregion. Immunsuppression bzw. Neutropenie (maligne Erkrankungen) gelten als primäre Risikofaktoren. Die Letalität ist hoch, jedoch stark abhängig von Prädisposition und Dauer der Infektion bis zum Therapie- 
beginn. Wir konnten dermale Mikrothrombosen auf Grund einer primären oder sekundären Gerinnungsstörung nicht ausschließen, weshalb eine hämostaseologische Vorstellung empfohlen wurde.

\section{DGKJ-PO 27 \\ Neurodermitis oder primärer Immundefekt? - Die diagnostische Herausforderung Ekzem}

Beate Hagl', Benedikt D Spielberger ${ }^{2}$, Renate Effner', Gundula Notheis ${ }^{3}$, Ulrich A Schatz ${ }^{8}$, Simon Rothenfusser ${ }^{4}$, Nina Schnopp ${ }^{5}$, Kilian Eyerich ${ }^{5}$, Claudia Traidl-Hoffmann', Michael Frühwald ${ }^{6}$, Thomas Meitinger ${ }^{8}$, Ellen Renner ${ }^{7}$ ${ }^{1} T U$ München und Helmholtz Zentrum München, Lehrstuhl und Institut für Umweltmedizin, UNIKA-T, München, Deutschland;'²T München und Helmholtz Zentrum München, Universitätskinderklinik, AlbrechtLudwig Universität, Freiburg, Lehrstuhl und Institut für Umweltmedizin, UNIKA-T, München, Deutschland; ${ }^{3}$ TU München und Helmholtz Zentrum München; Klinik für Kinder und Jugendliche Universitätsklinikum Augsburg, Lehrstuhl und Institut für Umweltmedizin, UNIKA-T, Augsburg, Deutschland; ${ }^{4}$ Klinikum der Universität München, LMU München, Abteilung für Klinische Pharmakologie, München; ${ }^{5}$ TU München, Klinik und Poliklinik für Dermatologie und Allergologie, München, Deutschla nd;'Universitätsklinikum Augsburg, Klinik für Kinder und Jugendliche, Augsburg, Deutschland; ${ }^{7} T$ München und Helmholtz Zentrum München; Hochgebirgsklinik Davos und Christine-Kühne-Center für Allergie Forschung und Edukation (CK-CARE), Schweiz, Lehrstuhl und Institut für Umweltmedizin, UNIKA-T, München, Deutschland; ${ }^{8}$ TU München und Helmholtz Zentrum München, Institut für Humangenetik, München, Deutschland

Einige primäre Immundefekte - wie die Hyper-IgE Syndrome (HIES) präsentieren sich neben rezidivierenden Infektionen mit Ekzem und erhöhtem Serum-IgE und zeigen so eine signifikante Überlappung mit atopischen Erkrankungen, insbesondere der Neurodermitis.

Im Gegensatz zu den meisten atopischen Erkrankungen, sind HIES monogene Krankheiten, die z. B. durch heterozygote Mutationen im Gen STAT3 (STAT3-HIES) oder homozygote Mutationen in DOCK8 (DOCK8-HIES) verursacht werden.

Obgleich große Fortschritte in der genetischen und funktionellen Molekulardiagnostik die Diagnosestellung deutlich verbessert haben, gibt es weiterhin Patienten, bei denen die Diagnose erst nach Manifestation irreversibler Komplikationen gestellt wird.

Anhand von klinischen Beispielen illustrieren wir die Herausforderung, monogene Ursachen bei HIES-Patienten zu identifizieren und als krankheitsverursachend zu bestätigen. Hierbei helfen uns im Fall von STAT3HIES die Untersuchung des Interleukin 6-spezifischen Signalwegs inklusive der TH17-Zellbestimmung im peripheren Blut. Allerdings können sich Mutationen, die die gleiche Aminosäure betreffen, funktionell unterschiedlich auswirken. Des Weiteren können genetische Varianten mit unklarer funktioneller Bedeutung, wie z.B. intronische Mutationen in DOCK8, können teils erst durch eine komplexe Kombination von klinischen, immunologischen, molekulargenetischen und bioinformatischen Ansätzen als krankheitsverursachend bestätigt werden.

Die Klärung von Krankheitsursachen auf funktionell-molekularer Ebene ist nicht nur für die frühzeitige Diagnose wichtig, sondern auch als Modell für die Entstehungsmechanismen von Volkskrankheiten (z. B. allergische oder onkologische Erkrankungen), bei denen es gilt, die erhöhte Vulnerabilität gegenüber Umwelteinflüssen besser zu verstehen.

\section{DGKJ-PO 28}

\section{Ein Praktikum mit Folgen}

Josephine Mohn

Universitätsklinikum Carl Gustav Carus Kinderklinik, Kinder- und Jugendmedizin, Dresden, Deutschland

Anamnese: 17-jähriger Junge mit seit 5 Tagen bestehendem Fieber, Kopfschmerzen, Gliederschmerzen und anfallsartigem Husten. Aktuell FSJ in
Frankreich, dort Arbeit in einem Umweltprojekt. Einnahme von Aspirin, PCM und Ibuprofen.

Befund: Reduzierter AZ, milde Tachypnoe, abgeschwächtes AG über rechtem Mittelfeld, Abdomen weich, keine Hepatosplenomegalie, neurologisch unauffälliger Befund.

Labor: CrP 108 mg/l, Procalcitonin 3,3, ng/ml, Thrombozyten 50 GPT/ 1, Myoglobin $136 \mu \mathrm{g} / \mathrm{l}$, Kreatinin $197 \mu \mathrm{mol} / \mathrm{l}$, Harnstoff 15,5 mmol/1, Natrium $127 \mathrm{mmol} / \mathrm{l}$, Makrohämaturie, HK bis 0,23, ALAT 1,47 $\mu \mathrm{mol} /\left(\mathrm{s}^{\star} \mathrm{l}\right)$, ASAT $3,93 \mu \mathrm{mol} /\left(\mathrm{s}^{\star} \mathrm{l}\right)$

Normwerte für weiteres Diff. BB, Drogenscreening, Astrup, Gerinnung. Serologie: Chlamydien/Mykoplasmen, Hantavirus negativ, LeptospirenAK im Serum (1:640) BfR Berlin: Leptospira-IgG/IgM positiv (ELISA) und LipL32 PCR (Urin) positiv

Echo o. p.B, RöThorax: Pneumonie re, Hilusreaktion, im Verlauf Befundzunahme mit bipulmonaler Infiltration und Pleuraergüssen, Sono Abdomen: Nephropathie mit Nierenschwellung bds., Splenomegalie, Sono Thorax: Pleuraerguss max $1 \mathrm{~cm}$, Konsolidierung basaler Lungenabschnitte.

Klinischer Verlauf: Sauerstoffbedarf bei Desaturationen bis min. $89 \%$, arterielle Hypotension (Bedarf von Volumensubstitution und Katecholaminen), Oligurie bei Rhabdomyolyse im Sinne eines akuten Nierenversagens, Hämatokritabfall, Thrombozytopenie, antibiotische Anfangstherapie mit Ampicillin/Sulbactam und Doxycyclin, später unter Verdacht einer Sepsis Ceftriaxon, darunter Stabilisierung des Allgemeinzustandes.

Schlussfolgerung: Die Leptospirose ist eine in Europa und vor allem bei Kindern- und Jugendlichen sehr seltene Erkrankung und führt häufig zum Multiorganversagen, welches dem klinischen Bild einer Sepsis entspricht. Der Fall zeigt, wie wegweisend eine ausführliche (Reise-)Anamnese ist! Eine mögliche Kontamination mit Leptospiren erfolgte wahrscheinlich bei der Reinigungsarbeit eines Flusses.

\section{Literatur}

1. Lopes AA, Costa E, Costa YA, Sacramento E, de Oliveira Junior AR, et al. (2004) Comparative study of the in-hospital case-fatality rate of leptospirosis between pediatric and adult patients of different age groups. Rev Inst Med Trop, Sao Paulo 46: 19-24.

\section{DGKJ-PO 29}

\section{Hämorrhagisches Fieber mit renalem Syndrom (HFRS) durch Puumala Virus - Eine seltene Infektionskrankheit?}

Maria Liapi', Georgia Avrami², Norbert Jorch', Eckard Hamelmann ${ }^{2}$

'Evagelisches Klinikum Bethel, Kinderzentrum, Bielefeld,

Deutschland;'Evangelisches Klinikum Bethel, Kinderklinik, Bielefeld,

Deutschland;

Hantaviren sind behüllten Einzel-Strang RNA Viren und gehören zu den zoonotischen Erreger. Die Viren sind weltweit verbreitet und werden von asymptomatischen infizierten Nagetieren übertragen. Hantaviren können unterschiedliche Krankheitsbilder auslösen.

Das Hämorrhagische Fieber mit renalem Syndrom (HFRS) in Asien und Europa und das Kardiopulmonale Syndrom (HCPS) in Amerika sind die wichtigsten Hantavirus-assoziierte klinischeSyndrome. Die Pathogenese der Hantavirus-Infektionen ist auf eine Zytokinen Überproduktion zurückzuführen. Die in Europa aufgetretene Hantavirus Infektionen werden überwiegend durch den Puumala Virus verursacht.

Es wird der Fall eines 15jährigen Jungen vorgestellt der sich mit dem Bild eines protrahierten grippalen Infektes stationär aufgenommen wurde. Im Verlauf entwickelte er eine akute Niereninsuffizienz mit Nephromegalie, Proteinurie, arterielle Hypotonie und Flüßigkeitsretention (Aszites, Pleuraergüsse, Perikarderguss). Dazu Blutbild-Veränderungen mit Leukozytose, Polyglobulie und schwerer Thrombozytopenie. Anamnestisch berichtete der Patient vom Zelten im Wald 3 Wochen vor Fieberbeginn, ein kürzlicher Auslandsaufenthalt bestand nicht. Serologisch liessen sich IgM und IgG Antikörper gegen Puumala Virus nachweisen. Der Patient zeigte einen unkomplizierten Verlauf eines HFRS. Bei fehlender kausalen Therapie wurde der Junge symptomatisch behandelt. Eine Nierendialyse war 
nicht erforderlich, innerhalb von 5 Wochen wurde ein Restitutio ad integrum erreicht.

Hantaviren Infektionen werden von asymptomatischen Nagetieren durch Aerosol-Inhalation übetragen. Der in Deutschland am häufigsten Puumala Virus kann zu dem Krankheitsbild eines HFRS (hämorrhagisches Fieber mit renalem Syndrom) führen mit insgesamt milden bis moderaten Verläufe und gute Prognose. Der Verlauf eines HFRS wird durch 5 verschiedenen Phasen (febrile, hypotensive, oligurische, polyurische und rekonvaleszenzphase) mit typischen klinischen und laborchemischen Veränderungen gekennzeichnet. Die Diagnose erfolgt durch die Bestimmung von PUUV-Antikörper im Serum.

Initial imponiert ein HFRS als eine banale grippale Infektion. Da der Verlauf häufig mild ist, ist es anzunehmen, dass viele Hantavirusinfektionen unentdeckt bleiben.

\section{Literatur}

1. Jiang $\mathrm{H}$ et al (2016) Hemorrhagic fever with renal syndrome:pathogenesis and clinical picture. Front. Cell Infect Microbiol :

2. Jiang $H$, Zheng $X$ (2017) Hantavirus infection: a global zoonotic challenge. Virol Sin 32(1):32-43

3. R. Ulrich et al. (2004) V

\section{DGKJ-PO 30}

ARDS bei einer Fünfjährigen mit chronischer Eisenmangelanämie - was steckt dahinter?

Sarah Kotsias-Konopelska', Susanne Lau², Beatrix Schmidt', Doris Staab², Horst von Bernuth ${ }^{4}$ Elena auf dem Brinke ${ }^{3}$, Jonathan Gross ${ }^{3}$

'St. Joseph Hospital, Kinderklinik, Berlin, Deutschland; ${ }^{2}$ Charité Universitätsmedizin Berlin, Pulmonologie, Berlin, Deutschland;'35t. Joseph Hospital, Pädiatrie, Berlin, Deutschland; ${ }^{4}$ Charité - Universitätsmedizin Berlin, Immunologie, Berlin, Deutschland

Fragestellung: Wir berichten über ein fast 6-jähriges Mädchen, das im Abstand von vier Wochen zweimalig auf Grund akuter respiratorischer Globalinsuffizienz mit Fieber und anamnestisch blutigem Erbrechen intensivmedizinisch behandelt wurde. Bei primärem Verdacht auf eine Infektion konnten Gruppe-A-Streptokokken, Adenoviren und Rhinoviren nachgewiesen werden. Der wiederholte akute Beginn sowie die dann überraschend schnelle Erholung vom ARDS mit CPAP-Beatmung zur klinischen Beschwerdefreiheit standen im Gegensatz zu persistierend ausgeprägten Befunden der radiologischen Bildgebung.

Die Patientin ist ein ehemaliges Drillingsfrühgeborenes mit postpartalem RDS II ${ }^{\circ}$. Sie sei in den Folgejahren bis auf eine einmalige Unterlappenpneumonie pulmonal gesund gewesen. Seit dem 18. Lebensmonat bestehe eine milde Eisentherapie-refraktäre mikrozytäre hypochrome Anämie mit V.a. Eisenverwertungsstörung nach weitreichender ambulanter $\mathrm{Ab}$ klärung. Zudem bestünden eine Fruktosemalabsorption, rezidivierende Bauchschmerzen sowie regelmäßige Exposition zu Schimmelpilzen, Vögeln und Reptilien.

Zur differentialdiagnostischen Abklärung (auto-)immunologischer sowie interstitieller und struktureller pulmonaler Grunderkrankungen erfolgten eine Lungen-CT sowie eine Bronchoskopie, welche den Verdacht auf eine pulmonale Hämosiderose erbrachte. Dies wurde im Verlauf zytologisch bestätigt.

Material und Methodik: Körperliche und laborchemische Untersuchungen, sonographische und radiologische Bildgebung, Endoskopie, Bronchoskopie und zytologische Untersuchung.

Ergebnisse: Bronchoskopie: Hyperäme Bronchialschleimhaut trotz Anämie. Bronchoalveoläre Lavage: Blutige zweite Fraktion, Nachweis von Siderophagen. Anhand dieser Ergebnisse wurde die Diagnose einer pulmonalen Hämosiderose gestellt.

Diskussion: Die Symptomatik bei pulmonaler Hämosiderose kann akut schubweise auftreten, durch Atemwegsinfekte getriggert werden und in Kombination mit diesen eine hochakute pulmonale Infektion imitieren. Das anamnestische Bluterbrechen muss restrospektiv als Bluthusten gewertet werden. Die klinische Trias aus mikrozytärer hypochromer An- ämie, Bluthusten und auffälligen radiologischen Befunden sowie der Siderophagennachweis in der bronchoalveolären Lavage wiesen auf eine pulmonale Hämosiderose hin. Bei leichter Sensibilisierung gegen Kuhmilch und einer Körperlänge $<5$. Perzentile wurde differentialdiagnostisch an ein Heiner Syndrom gedacht, erscheint hier aber nicht wahrscheinlich. Zeitnah wurde eine Pulstherapie mit Methylprednisolon begonnen. Die Patientin befindet sich aktuell im zweiten Therapiezyklus in gutem Allgemeinzustand.

Schlussfolgerung: Die pulmonale Hämosiderose ist eine seltene und unterschiedlich akut verlaufende Erkrankung, an die bei Kindern mit therapierefraktären hypochromen mikrozytären Anämien, Bluthusten und radiologischen Hinweisen auf Hämorrhagien („Crazy paving Muster“) gedacht werden sollte. Die idiopathische pulmonale Hämosiderose ist eine Ausschlussdiagnose.

\section{DGKJ-PO 31}

\section{„Fehlende" Eosinophilie - Parasitose ausgeschlossen?}

Niklas Janssen ${ }^{1}$, Ralf-Bodo Tröbs', Richard Viebahn' ${ }^{2}$, Peter Seiffert ${ }^{3}$ ${ }^{1}$ Helios Klinikum Duisburg, Klinik für Kinderchirurgie, Duisburg, Deutschland; ${ }^{2}$ Knappschaftskrankenhaus Bochum, Klinik für Chirurgie, Bochum, Deutschland; ${ }^{3}$ Helios Klinikum Duisburg, Klinik für Kinder- und Jugendmedizin, Duisburg, Deutschland

- Fallbericht -

Einleitung: Häufigste chirurgische Baucherkrankung beim Schulkind ist die Appendizitis. Infolge eines weltweiten Tourismus und der Migration sind weitere Krankheitsbilder ins Kalkül zu ziehen. Ein Blutbild kann hierzu wertvolle Informationen liefern. Insbesondere bei Verdacht auf Parasitose erwartet man eine Erhöhung der eosinophilen Granulozyten.

Fallbericht: Das 8-jährige Mädchen aus Syrien wurde unter dem Verdacht auf eine Appendizitis eingewiesen. Im Vorfeld hatte das Mädchen einen Sturz erlitten und entwickelte Bauchschmerzen, Erbrechen sowie dünnen Stuhlgang. Überraschend ergaben Sonografie und MRT neben viel freier Flüssigkeit mehrere große Leberzysten, manche mit einer randständigen Membran. Einige Zysten waren flüssigkeitsfrei und erschienen kollabiert. Bei beeinträchtigtem Allgemeinzustand erfolgten Laparoskopie sowie die Absaugung der freien Flüssigkeit. Die Peritonealzytologie ergab 62.000 Zellen/ $\mu$, davon 30 \% Eosinophile. Dagegen war die PCR auf Echinococcus negativ. Im Blutbild entwickelte sich postoperativ eine Leukozytose von $29.000 / \mu \mathrm{lmit} 39 \%$ eosinophilen Granulozyten und zudem belegte die die Serologie eine Infektion mit Echinococcus granulosus [Echinococcus granulosus Titer (IHA) 1:5120, IgG-Antikörper (EIA) positiv]. Wir begannen eine Langzeit-Behandlung mit Albendazol (16 mg/kg/Tag). Sechs Wochen nach Beginn der systemischen Therapie erfolgte die multiple Enukleation der Endozysten nach vorheriger intraoperativen Instillation mit 20 \%-iger Kochsalzlösung. Das histologische Bild bestätigte eine Echinokokkose - vitale Erreger waren nicht nachweisbar. Der postoperative Verlauf war unspektakulär und regelmäßige sonografische Kontrollen unter Albendazol zeigten einen nahezu vollständigen Befundrückgang im Laufe eines Jahres.

Diskussion: Trotz des ausgeprägten parasitären Befalles fand sich im initialen Blutbild keine richtungsweisende Auffälligkeit (Leukozytenzahl 10.760/ $\mu$ l, eosinophile Gran. 7,3 \%). Erst der peritoneale Kontakt des Zysteninhaltes nach Zystenruptur führte zur Eosinophilie. Ein in diesem Zusammenhang gefürchteter anaphylaktischer Schock trat nicht ein.

Schlussfolgerung: Bei Nachweis von Leberzysten schließt ein unauffälliges Differentialblutbild das Vorliegen einer Echinokokkose nicht aus. Pathognomonisch für die Echinokokkose waren die Bildgebung sowie die Serologie.

Es bestehen keine Interessenskonflikte. 


\section{Kardiologie/Nephrologie}

\section{DGKJ-PO 33 \\ Die prognostische Bedeutung von Troponin T bei pädiatrischem Polytrauma}

Christian Karl Braun', Annika Schaffer ${ }^{2}$, Miriam Kalbitz' ${ }^{2}$ Markus Huber-Lang', Jochen Preßmar

'Universitätsklinikum Ulm, Institut für Klinische und Experimentelle Trauma-Immunologie, Ulm, Deutschland;'Universitätsklinikum Ulm, Klinik für Unfall-, Hand-, Plastische und Wiederherstellungschirurgie, Ulm, Deutschland

Einleitung: Schwere Verletzungen stellen weltweit die häufigste Todesursache im Kindes- und Jugendalter dar. Bei Erwachsenen mit schwerem Gewebetrauma konnte in Vorarbeiten eine prognostische Bedeutung der Bestimmung von herzspezifischem Troponin bei Schockraumaufnahme gezeigt werden. Ob Troponin bei polytraumatisierten Kindern eine ähnliche Bedeutung zukommt, und die Bestimmung von Troponin T bei Schockraumaufnahme das klinische Management verbessern kann, ist bisher nicht erforscht. Wir präsentieren Daten einer restrospektiven, monozentrischen Studie über Kinder und Jugendliche mit Polytrauma, die in einem Level-1 Traumazentrum behandelt worden sind.

Material und Methoden: Die klinischen und laborchemischen Daten von 88 pädiatrischen Patienten, die mit Polytrauma in unserer Klinik für Unfallchirurgie des Universitätsklinikums Ulm im Zeitraum von 2007 bis 2016 behandelt worden sind, wurden retrospektiv ausgewertet. Die Patienten wurden nach Werten des Injury Severity Scores in drei Gruppen für leichtes, mittelschweres und sehr schweres Trauma stratifiziert. Die laborchemisch bestimmten Parameter Troponin T (TnT), Interleukin-6 (IL-6) und die Creatinkinase-Aktivität (CK-Aktivität) sowie Laktat wurden unter den stratifizierten Gruppen verglichen, mit klinischen Parametern korreliert und prognostische und diagnostische Bedeutung von TnT und IL-6 statistisch ausgewertet.

Ergebnisse: Patienten in der Gruppe mit schwerem Polytrauma zeigten signifikant erhöhte Troponin-T Werte verglichen mit Patienten mit leichtem und mittelschwerem Trauma. TnT-Werte über einem Cut-off von $14 \mathrm{ng} /$ $\mathrm{mL}$ waren signifikant assoziiert mit radiologisch-diagnostizierten Lungenkontusionen, erhöhtem Sequential-Organ Failure Score (SOFA-Score) und tödlichem Verlauf nach Klinikaufnahme. Patienten mit Thorax-Trauma, Lungenkontusionen, Multi-Organversagen und Nicht-Überlebende hatten signifikant erhöhte absolute TnT-Plasmawerte. In unserer Studie war TnT bei Schockraumaufnahme in dieser Hinsicht IL-6 prognostisch überlegen.

Diskussion: TnT im Plasma korrelierte in unserem Patientenkollektiv bereits bei Schockraumaufnahme mit der Schwere des Traumas, unabhängig von einer klinisch messbaren Beeinträchtigung der Herzfunktion. Daten aus dem Tiermodell konnten eine frühe Schädigung von Herzmuskelzellen nach experimentellem Trauma auf molekularer Ebene zeigen, ohne dass eine direkte Organschädigung morphologisch nachweisbar war. Die Bedeutung dieser subzellulären Schädigung ist bisher noch unklar. Unsere Daten legen eine prognostische Bedeutung von herzspezifischem Troponin für den klinischen Verlauf nahe. Hinsichtlich des positiv prädiktiven Wertes war es in unserer Studie dem etablierten frühen Entzündungsmarker IL-6 überlegen.

Schlussfolgerung: Die Bestimmung von herzspezifischem Troponin kann bei Schockraumaufnahme dazu beitragen, die Schwere des Gewebetraumas und den klinischen Verlauf bei polytraumatisierten Kindern und Jugendlichen abzuschätzen.
DGKJ-PO 34

Analyse der körperlich-sportlichen Aktivität im Alltag von Jugendlichen und jungen Erwachsenen mit angeborenen Herzfehlern

Miriam Heinzelmann', Claudia Niessner², Jannos Siaplaouras³, Christian Apitz $^{3}$

'Universität Ulm, Sektion Pädiatrische Kardiologie, Ulm, Deutschland:²Karlsruher Institut für Technologie, Institut für Sport und Sportwissenschaften, Karlsruhe, Deutschland; ${ }^{3}$ Universitätsklinik für Kinder- und Jugendmedizin Ulm, Sektion Pädiatrische Kardiologie, Fulda, Deutschland;

Hintergrund: Dank der Weiterentwicklung der medizinischen Versorgung in den letzten Jahrzehnten sank die Letalität von Patienten mit angeborenem Herzfehler (AHF) deutlich, gleichzeitig nimmt die Zahl der jugendlichen und erwachsenen Patienten mit AHF stetig zu. Hiermit gewinnt die Prävention erworbener Erkrankungen und kardiovaskulärer Risikofaktoren durch körperlich-sportliche Aktivität (KSA) an Relevanz. Bisherige Daten über die KSA bei Jugendlichen und jungen Erwachsenen mit AHF und der damit verbundenen Körperwahrnehmung sind limitiert. Fragestellung: Ziel der vorliegenden Studie ist es, den Ist-Zustand der KSA von Jugendlichen und jungen Erwachsenen mit AHF mit der Normalbevölkerung zu vergleichen. Es wird beantwortet, ob der Schweregrad des AHF einen Einfluss auf das Aktivitätsverhalten, die persönliche Einstellung zur KSA sowie die Eingliederung in sportbezogene Betreuungsstrukturen hat.

Material und Methode: Das körperlich-sportliche Aktivitätsverhalten von Patienten mit einfachen (EAHF; $n=66$ ) und komplexen AHF (KAHF; $n=35$ ) wurde in einem prospektiven monozentrischen Studiendesign mittels zweier Fragebögen zum Aktivitätsverhalten und zur Betreuung mit einem für Deutschland repräsentativen Normkollektiv (NK; $n=1409)$ verglichen.

Ergebnisse: Es zeigte sich ein signifikanter Unterschied in der Gesamtaktivität der Gruppen $(p<0,001)$. Die Patienten mit EAHF waren mit 4,4 Aktivitätstagen á 60 min pro Woche aktiver als die Patienten mit KAHF und auch aktiver als das NK (3,1 und 3,4 Aktivitätstage á $60 \mathrm{~min}$ pro Woche). Die Patienten mit EAHF und das NK betrieben mit $53 \%$ mehrheitlich mindestens eine Vereins- oder Freizeitsportart, während die Patienten mit KAHF mit $45 \%$ zum Großteil keinen Sport betrieben.

Das NK erreichte in allen sechs Kategorien des physischen Selbstkonzeptes (Koordination, Ausdauer, allgemeine Sportlichkeit, Kraft, Beweglichkeit, Schnelligkeit) Werte eines positiven Selbstbildes, die Patienten mit EAHF zeigten ein überwiegend positives oder indifferentes Selbstbild, während die Patienten mit KAHF in vier Kategorien ein negatives physisches Selbstkonzept aufwiesen. Es ließ sich weiterhin ein signifikanter Zusammenhang zwischen dem physischen Selbstkonzept und der Aktivität bzw. der Teilhabe an Vereins- und Freizeitsport nachweisen $(p<0,001)$. Hinsichtlich sportbezogener Betreuungsstrukturen zwischen den Gruppen konnte kein signifikanter Unterschied gezeigt werden.

Schlussfolgerung: Patienten mit EAHF sind ähnlich aktiv wie die Normalbevölkerung und scheinen gut integriert in Sportvereine. Dagegen zeigen Patienten mit KAHF ein deutlich geringeres Interesse an Sport, sind weniger körperlich aktiv und nur vereinzelt integriert in Sportvereine, was mit einem negativen Selbstbild bzgl. der eigenen Körperwahrnehmung korreliert. Da insbesondere Patienten mit KAHF von einer, auf ihre Leistungsfähigkeit abgestimmten, sportlichen Betätigung profitieren, gilt es zukünftig, die körperliche Aktivität speziell dieser Gruppe zu fördern.

\section{DGKJ-PO 35}

Therapie der arteriellen Hypertonie bei apparentem Mineralkortikoidexzess eines Jugendlichen mit Non-Compliance

Ann-Kathrin Nagel', Anthea Peters', Johannes Breuer', Bernd Hoppe ${ }^{2}$

'Universitätskinderklinik Bonn, Kinderkardiologie, Bonn, Deutschland;

${ }^{2}$ Universitätskinderklinik Bonn, Kindernephrologie, Bonn, Deutschland 
Hintergrund: Der apparente Mineralkortikoidexzess (AME) ist eine seltene Ursache einer therapierefraktären arteriellen Hypertonie. Für die autosomal-rezessiv vererbte Erkrankung sind bisher weniger als 100 Fälle beschrieben. Durch Aktivitätsminderung der 11-ß-Hydroxysteroiddehydrogenase kommt es zur verminderten Umwandlung von Cortisol in Cortison und damit konsekutiv zur Überaktivierung des Mineralkortikoidrezeptors mit der Folge eines Pseudohyperaldosteronismus. Symptome sind Gedeihstörung, arterielle Hypertonie und eine Nephrokalzinose. Unbehandelt besteht durch die maligne Hypertonie sowie renale und kardiale Insuffizienzen eine schlechte Prognose. Schlaganfälle bereits vor dem 10. Lebensjahr sind häufig.

Fallbericht: Wir berichten von einem 15 Jahre alten Jungen aus Katar (Körperlänge und -größe $<1$. Perzentile) mit genetisch gesicherten AME (homozygote Variante im Exon 2 HSD11B2), der sich mit therapierefraktärem Hypertonus sowie Nephrokalzinose zur Therapieoptimierung vorstellte. Laborchemisch zeigte sich bei Aufnahme eine metabolische Alkalose $(\mathrm{pH}$ $7,46, \mathrm{~K}+2,24 \mathrm{mmol} / \mathrm{l})$. Die 24-h-Blutdruckmessung ergab unter der bestehenden Medikation mit Amlodipin, Hydrochlorothiazid und Lisinopril eine arterielle Hypertonie $\mathrm{II}^{\circ}$ bei einem mittleren Wert von $145 \mathrm{mmHg}$ systolisch, $90 \mathrm{mmHg}$ diastolisch (jeweils $>99$. Perzentile $+5 \mathrm{mmHg}$ ) und ein nächtliches Non-Dipping. Als Korrelat hierzu zeigte sich echokardiographisch eine mäßige linksventrikuläre Myokardhypertrophie. Trotz bekannter Diagnose des AME erfolgte die antihypertensive Therapie bisher ohne die Gabe eines Mineralkortikoidrezeptorantagonisten als Kausaltherapeutikum des Enzymdefektes.

Nach Gabe von Spironolacton zusätzlich zur bestehenden Medikation sanken die Blutdruckwerte in altersentsprechende Bereiche. Die Hypokaliämie wurde zusätzlich mit einer oralen Kaliumsubstitution therapiert. Röntgenologisch stellte sich eine vollständige Verknöcherung der Epiphysenfugen und somit keine Option für eine Wachstumshormontherapie dar.

Nach Entlassung kam es im Verlauf zur erneuten Vorstellung in unserer Notfallambulanz bei hypertensiver Entgleisung mit Werten bis $160 \mathrm{mmHg}$ systolisch. Als Ursache stellte sich nun eine unregelmäßige Medikamenteneinnahme heraus. Zur Erhöhung des Compliance vereinfachten wir das Therapieschema mit Dosiserhöhung von Spironolacton (50 mg p.o. $2 \times$ tägl.) als Kausaltherapie und konnten auch so altersentsprechende RRZielwerte erreichen.

Schlussfolgerung: Bei therapierefraktärer Hypertonie muss auch an seltene genetische Ursachen gedacht werden und diese bei Vorliegen gezielt behandelt werden. Aufgrund der schlechten Prognose durch die Folgen der malignen Hypertonie sollte bei der symptomatischen Therapie des AME die Senkung des Blutdrucks im Vordergrund stehen. Insbesondere bei Noncompliance sollte deshalb ein hoher Wert auf den Entwurf einfacher Therapieschemata und deren Einhaltung gelegt werden.

\section{DGKJ-PO 36}

Prävalenz und Schweregrad von hohem Blutdruck bei Kindern basierend auf den neuen Guidelines der American Academy of Pediatrics(AAP) 2017: Überprüfung und Bedeutung der Praxisauswirkungen nach den Sharma-Daten, Manitoba, Canada 2018

Richard Eyermann ${ }^{1,2}$

'Kinder- und Jugendmedizin, Kinderkardiologie, Sportmedizin, München, Deutschland; '2Klinik Schönsicht Berchtesgaden, Rehabilitation für Kinder und Jugendliche, AHB, Kind-Mutter/Vater-Rehabilitation, München, Deutschland

Bedeutung: Basierend auf den neuen Blutdruckguidelines von 2017 ist die Prävalenz von Bluthochdruck bei Erwachsenen von 32 auf $46 \%$ gestiegen. Basierend auf neuen Normen u. diagnostischen Schwellenwerten, die sich besser an die Definitionen von Adulten anpassen, wurden neue Leitlinien für die klinische Praxis für Kinder veröffentlicht. Die Richtlinien für die klinische Praxis der AAP für das Management von erhöhtem RR bei Kindern ersetzen den 4th Report der Task Force des NHLBI von 2004.
Ziele: Beurteilung der Konsequenzen der klinischen Praxisguidelines der AAP für das Management von erhöhtem RR bei Kindern auf die Prävalenz u. Schwere des erhöhten RR bei Kindern u. die Charakterisierung von Risikofaktoren für Kinder mit neu auftretender Hypertonie oder einer Verschlechterung im klinischen Stadium („Nach oben umklassifiziert"). Design, Setting, Probanden: Diese Studie wendete beide Richtlinien an, um RR in 15.647 allgemein gesunde Kinder mit niedrigem Risiko im Alter von 5 bis 18 Jahren aus nationalen Gesundheits- $u$. Ernährungsuntersuchungen (vom 1. Januar 1999 bis 31. Dezember 2014) einzustufen. Im Fall-Kontrollteil der Studie wurden Kinder, deren RR nach oben reklassifiziert wurde (Fälle), hinsichtlich Geschlecht, Alter u. KL mit Kontrollen mit normalem RR verglichen. Die anthropometrischen u. laboratorischen Risikofaktoren wurden verglichen u. die alters- u. geschlechtsspezifischen Z-Werte für KG, TU u. BMI berechnet. Der RR wurde durch Auskultation durch geschultes Personal gemessen. Nachdem sich das Kind 5 min ruhig erholt hatte, wurden 3 bis 4 aufeinanderfolgende RRwerte aufgezeichnet. Wichtige Ergebnisse: Perzentile des RR u. klinische Einstufung basierend auf den Guidelines der AAP 2017 u. dem National Institute of Heart, Lung und Blood Institute(NHLBI) von 2004.

Unter den 15.647 Kindern in der Studie (7799 Mädchen u. 7848 Jungen; Durchschnittsalter [SD], 13,4 [2,8] Jahre), basierend auf den Richtlinien der AAP, stieg die geschätzte (gewichtete) Bevölkerungsprävalenz von erhöhtem RR von 11,8 \% (95 \% CI, 11,1-13,0 \%) auf 14,2 \% (95 \% CI, 13,4$15,0 \%)$. Insgesamt hatten 905 von 15.584 Kindern $(5,8 \%)$ eine neu diagnostizierte Hypertonie $(n=381)$ oder eine Verschlechterung im klinischen Stadium ( $n=524)$, was eine erhebliche Zunahme der Krankheitslast für das Gesundheitssystem bedeutet. Kinder, deren RR nach oben umklassifiziert worden war, waren mehr übergewichtig oder adipös, mit höheren z-Scores für KG, TU u. BMI. Die Prävalenz von abnormalen Laborergebnissen war ebenfalls erhöht, mit negativen Lipidprofilen u. erhöhten HBA1c-Spiegeln (Prädiabetes).

Konklusion, Relevanz: Die Clusterbildung kardiovaskulärer Risikofaktoren bei ansonsten gesunden US-Kindern legt nahe, dass diejenigen, deren RR neu klassifiziert wurde, eine Population mit hohem Risiko darstellen, deren kardiovaskuläres Risiko zuvor möglicherweise unterschätzt wurde. Die ESC befürwortet für ihre Adulten keine Absenkung der RR-Bereiche (höhere Morbidität u. Mortalität).

\section{DGKJ-PO 37}

\section{Age-dependent changes of kidney injury biomarkers in pediatrics}

Tamara van Donge', Tatjana Welzel', Johannes N. van den Anker², Marc Pfister $^{1}$

'University Children's Hospital Basel (UKBB), University of Basel, Basel, Switzerland, Pediatric Pharmacology and Pharmacometrics, Basel, Switzerland;'2University Children's Hospital Basel (UKBB), University of Basel, Basel, Switzerland AND Children's National Health System Washington, DC, USA, Pediatric Pharmacology and Pharmacometrics AND Division of Clinical Pharmacology, Basel, Switzerland

Background: Currently used creatinine based parameters for monitoring of kidney function aren't reliable for early detection of kidney injury (KI), particularly tubular dysfunction. They also don't show the localization of KI. Several more recent KI biomarkers allow early detection of glomerular and tubular damage in adulthood. As optimized KI monitoring can prevent chronic kidney diseases in infancy, childhood and adolescence, the goal of this research project was to establish reference values for KI biomarkers in these age groups.

Material and methods: A systematic literature search was performed in PubMed. After elimination of duplicates, literature was screened for inclusion criteria (healthy participants, age $\leq 20$ years, clinical trial, report of absolute values for KI biomarkers, full text available, English language) and exclusion criteria (nephrotoxic therapy, artificial ventilation, co-morbidities, infections). Reported values of KI biomarkers were plotted by gestational age for preterm neonates and by age groups in infants, children and adolescents. 
Results: A total of 12 out of screened 205 studies met predefined criteria and included 219 preterm neonates (gestational age $<36$ weeks) and 2392 term neonates, infants, children and adolescents. KI biomarkers were analyzed in urine ( 6 studies), in serum/plasma (5 studies) and in serum and urine ( 1 study). In these studies, $6 \mathrm{KI}$ biomarkers were investigated in serum/plasma and 26 in urine. Four studies $(\mathrm{N}=555)$ measured the urinary KI biomarker Kidney Injury Marker-1 (uKIM-1) [1-4], five studies $(\mathrm{N}=888)$ the urinary KI biomarker Neutrophil Gelatinase-Associated Lipocalin (uNGAL) [1-5], and two studies $(\mathrm{N}=203)$ the serum KI biomarker Cystatin C (sCysC) $[6,7]$. Exploratory graphical analysis indicated that values for uNGAL, uKIM- 1 and sCysC had higher values in preterm neonates than in infants, children or adolescents. Furthermore, uNGAL and UKIM-1 values slightly increase around the age of 15 to 18 years.

Discussion: This literature review of KI biomarkers in different pediatric age groups indicates that (i) the majority of KI biomarkers are measured in urine, (ii) the three most commonly analyzed KI biomarkers are $\mathrm{uN}$ GAL, uKIM-1 and sCysC, (iii) values of KI injury biomarkers seem to be age-dependent, (iv) and that there is a need to further enhance knowledge about age-dependent changes of KI biomarkers in pediatrics.

Conclusion: Values of the key KI biomarkers such as UNGAL, uKIM- 1 and sCysC appear to decrease from prematurity to infancy. Additional studies are warranted to better characterize reference values for these key KI biomarkers across pediatric age groups and to evaluate the value of such markers in the early detection of drug related kidney injury in pediatric patients.

\author{
References \\ 1. Bennett. Pediatr Nephrol, 2015. 30(4) \\ 2. Saeidi. Pediatr Nephrol, 2015. 30(11) \\ 3. Bennett. Pediatr Rheumatol Onl J, 2017. 15(1) \\ 4. Zwiers. CCLM, 2015. 53(8) \\ 5. Cangemi. CCLM, 2013. 51(5) \\ 6. Armangil.Pediatr Nephrol, 2008. 23(11) \\ 7. Zwiers et al. CCLM, 2014. 52(12)
}

\section{DGKJ-PO 38}

\section{Therapeutische Apherese in pädiatrischen Dialyseeinrichtungen in Deutschland}

\section{Christina Taylan', Julia Thumfart², Lutz Weber', Dominik Müller}

'Universitätsklinik Köln, Kindernephrologie, Köln, Deutschland; ${ }^{2}$ Charité Universitätsmedizin, Klinik für Pädiatrie mit Schwerpunkt Nephrologie, Berlin, Deutschland; ${ }^{3}$ Universitätsklinikum Köln (ÄöR), Kindernephrologie, Köln, Deutschland

Hintergrund: Hauptindikationen für die therapeutische Apherese (TA) in der Pädiatrie sind nephrologische, neurologische und hämatologische Erkrankungen. Der klinische Einsatz der TA bei pädiatrischen Patienten ist bisher nicht systematisch dokumentiert. Ziel dieser Fallsammlung ist es, durch die Auswertung der Daten der TA weitere Erkenntnisse zur Wirksamkeit und Verträglichkeit der Therapie in der klinischen Routine bei Kindern und Jugendlichen zu erlangen und einen Beitrag zur Standardisierung des Einsatzes der TA in dieser Patientengruppe zu erstellen.

Methoden: Primärer Zielparameter der online-basierten, multizentrischen Registerstudie ist die Wirksamkeit der TA auf die jeweilige klinische Symptomatik der zugrundeliegenden Erkrankung. Dazu werden die Symptome vor und nach der TA, wenn möglich mit einem krankheitsspezifischen Score erfasst. Sekundäre Zielparameter sind die Sicherheit und Verträglichkeit der Behandlungen. 6-monatlich erfolgt eine follow-up Visite. Dokumentiert werden Patienten im Alter von 0-18 Jahren, die in den beteiligten Kliniken mit TA behandelt wurden (retrospektiver Teil), bzw. werden (prospektiver Teil). Die Auswertung der Daten soll die Erstellung von Standards zur Behandlung mit TA möglich machen. Zudem erfolgt damit eine zentrale Dokumentation der Wirksamkeit und Verträglichkeit der TA bei Kindern und Jugendlichen.

Ergebnisse: Das Register ist seit März 2018 online. Bis heute wurden an 9 Zentren ca. 280 Behandlungen für 16 Patienten eingegeben. Es zeigt sich eine sehr gute Verträglichkeit der TA (1 berichtete Komplikation „Erbrechen"). $43 \%$ der TA wurden aufgrund einer nephrologischen Grunderkrankung durchgeführt, $31 \%$ aufgrund neurologischer Grunderkrankungen, $6 \%$ wegen einer Autoimmunerkrankung. Bei $19 \%$ sind andere zugrunde liegende Erkrankungen dokumentiert. $52 \%$ der dokumentierten Behandlungen sind Immunadsorptionen, 48 \% Plasmaspheresen. $52 \%$ der Patienten wurden über eine AV-Fistel behandelt, nur $48 \%$ über einen zentralen Katheter. Die Anzahl, Frequenz und Dauer der Behandlungen sind trotz gleicher Indikation heterogen. Die Auswertung der ersten follow-up Visiten zeigt keine dokumentierte Verschlechterung der Symptome der Patienten, 2 Patienten mit akuter Behandlung erfuhren eine Restitutio ad integrum, 3 Patienten eine Verbesserung, 6 Patienten mit chronischer Behandlung zeigen eine gute Krankheitskontrolle unter laufender TA und für 5 Patienten liegt noch keine Nachuntersuchung vor.

Schlussfolgerung: TA ist ein sicher einzusetzendes, gut verträgliches Therapieverfahren, das in pädiatrischen Dialysezentren regelmäßig durchgeführt wird. Die Indikationen sind vielfältig, es fehlen Evidenz basierte Standards in der Diagnostik und der Behandlung, deren Entwicklung durch die Auswertung der Registerdaten ermöglicht werden kann. Weiterhin soll das Aphereseregister beim Aufbau eines bisher fehlenden Netzwerks zwischen der pädiatrischen Nephrologie und anderen Fachgesellschaften, die TA durchführen, helfen.

\section{DGKJ-PO 39}

\section{Primäre Hyperoxalurie Typ I}

Bettina Schulz', Simone Wygoda', Michael Pohl', Sabine Hollenbach ${ }^{2}$

${ }^{1}$ Klinikum St. Georg gGmbH, KfH Nierenzentrum für Kinder und Jugendliche, Leipzig, Deutschland;'KfH, Nierenzentrum für Kinder und Jugendliche, Leipzig, Deutschland

Einleitung: Die Primäre Hyperoxalurie Typ I ist eine sehr seltene, autosomal rezessiv vererbte, Stoffwechselstörung mit einer Prävalenz von 1:333.000-1.000.000. Sie beruht auf einem Defekt des peroxisomalen Leberenzyms L-Alanin-Glyoxylat-Aminotransferase, in dessen Folge Oxalat im Körper akkumuliert. Sie besitzt eine phänotypische Heterogenität, die von einer schweren infantilen Form mit raschem Eintritt einer terminalen Niereninsuffizienz über einen variablen und langsameren Verlauf im Kindes- und Jugendalter bis zu einer milden Spätform des Erwachsenen mit Nephrolithiasis und möglichem akuten Nierenversagen durch Steinobstruktion reicht.

Material/Methode: Falldarstellung eines 13-jährigen Patienten (Fußballspieler, $170 \mathrm{~cm}, 70 \mathrm{~kg}$ ), der wegen seit mehreren Wochen anhaltenden Erbrechens und Oberbauchschmerzen in eine auswärtige Kinderklinik akut aufgenommen wurde. Es zeigte sich eine schwerstgradige Urämie mit körperlicher Adaptation an ein Kreatinin von $4353 \mu \mathrm{mol} / \mathrm{l}$, Harnstoff von $85,3 \mathrm{mmol} / \mathrm{l}, \mathrm{pH}$ von 7,17, Bicarbonat von $12 \mathrm{mmol} / \mathrm{l}, \mathrm{BE}$ von - $15,3 \mathrm{mmol} /$ 1, Kalium von $6,9 \mathrm{mmol} / \mathrm{l}, \mathrm{Hb}$ von $3,5 \mathrm{mmol} / \mathrm{l}$, Hk von $16 \%$ und einer Serumosmolarität von $364 \mathrm{mosmol} / \mathrm{kg}$. Weiterhin waren Lipase und Herzenzyme erhöht.

Ergebnisse: Anamnestisch war bei dem Jungen ein Nierensteinleiden seit dem Alter von 9 Jahren bekannt (2014 operative Steinentfernung in einer urologischen Klinik). Rezidivierend hatte er Steinabgänge und „Grieß“ im Urin. Die ambulante Betreuung erfolgte bei einem Urologen. Nie wurden eine Stoffwechseldiagnostik oder eine kindernephrologische Vorstellung initiiert. Zuletzt zeigten sich 1 Monat vor der Krankenhausaufnahme ein Ureterstein (der spontan abging) und eine Nephrokalzinose. Es wurden, auch hinsichtlich der anhaltenden Oberbauchbeschwerden, keine Blutuntersuchungen veranlaßt.

Wir begannen die Hämodialysebehandlung und medikamentöse Einstellung der Sekundärfolgen des terminalen Nierenversagens. Weitere Befunde: sonografisch schwere Nephrokalzinose, Plasmaoxalat initial $165 \mu \mathrm{mol} / 1$ und Plasmaglykolat $543 \mu \mathrm{mol} / \mathrm{l}$, im Verlauf Plasmaoxalat vor Dialyse $109 \mu \mathrm{mol} / \mathrm{l}$ und nach $5 \mathrm{~h}$ Dialyse $39 \mu \mathrm{mol} / \mathrm{l}$.

Diskussion: Eine frühe intensive Behandlung oder präemptive Leber/ Nieren-Transplantation zur Verhinderung der Dialysepflichtigkeit waren nicht mehr möglich. Aktuell erhält der Patient den Versuch einer Pyri- 
doxin-Therapie und intensive Hämodialysen $6 \times$ pro Woche jeweils $5 \mathrm{~h}$. Die Verdachtsdiagnose wurde in der genetischen Untersuchung bestätigt. Fazit: Bei Nierensteinleiden im Kindesalter muß immer eine Stoffwechseldiagnostik erfolgen, um die Ursache zu erkennen und eine entsprechende Therapie einzuleiten. Hierfür ist die Vorstellung in einer kindernephrologischen Sprechstunde immer anzuraten.

\section{DGKJ-PO 40}

Komplizierte Harnwegsinfektionen im Kindesalter - ein Plädoyer für eine gute Vernetzung und die zukünftige Leitlinie

Holger Hauch ${ }^{1}$, Stephanie Goldammer ${ }^{2}$, Lucas Sichardt'ㄹ, Martin Kömhoff ${ }^{3}$, Carmen Knöppel', Stefanie Weber ${ }^{4}$

'UKGM Standort Gießen, KinderPalliativTeam Mittelhessen, Gießen, Deutschland; ${ }^{2}$ Klinikum Bad Hersfeld, Klinik für Kinder- und Jugendmedizin, Bad Hersfeld, Deutschland;'3KGM Standort Marburg, Klinik für Kinderund Jugendmedizin mit SP Nephrologie, Marburg, Deutschland; ${ }^{4}$ UKGM Standort Marburg, Klinik für Kinder- und Jugendmedizin, Marburg, Deutschland

Einleitung: Harnwegsinfektionen (HWI) gehören zu den häufigsten Infektionskrankheiten (1). Abszedierungen (A) oder Sepsis/SIRS (S) (4) sind akute Komplikationen (2). Ein Ziel der berichtenden Klinik in Bad Hersfeld ist es, universitäre Kompetenz durch Vernetzungen in der Regelversorgung (RV) zu verankern. Es erfolgte eine Auswertung von komplizierten HWI vom 01.02.2018-31.01.2019 zur Evaluation der Kooperation. Material und Methoden: Retrospektive Analyse der stat. Fälle nach $\$ 21$ KHEntgG(3). Alle Fälle mit HWI als HD wurden bzgl. akuter Komplikationen (A/S) analysiert. Bei Vorliegen einer Komplikation erfolgte die Auswertung der Krankenakte.

Ergebnisse: 2329 Kinder, 29 davon (1,2 \%) wurden stationär wegen HWI behandelt. 5 Kinder ( 4 w, 1 m, Alter: 3 M.-16 J.) zeigten Komplikationen. 3 Kinder hatten ein A und S, 1 Kind A und ein Kind ein S. Von 8 Urin-Parametern waren mind. 2 bis max. 4 pathologisch. CRP und ProcalcitoninWerte betrugen $38-345 \mathrm{mg} / \mathrm{l}$, bzw. 0,5-57,3 $\mu \mathrm{g} / \mathrm{l}$. In allen Fällen erfolgte eine Ultraschalluntersuchung zuzüglich CT bei V. a. Ileus $(n=2)$, MRT bei V.a. A $(n=2)$ und MCU z. A. eines VUR $(n=3)$. Pos. Keimnachweis in $4 / 5$ der Fälle (E. coli, $n=3 ; 1 \times$ Citrobacter koseri, $n=1$ ). Diagnosestellung innerhalb von 12 Stunden in allen Fällen. Initiale Behandlung erfolgte empirisch in 4/5 Fällen mit Ampicillin/Cefotaxim, bzw. 1/5 mit Ampicillin/Imipenem. Die i.v. Behandlungsdauer betrug zwischen 10 und 21 Tagen (d), die orale Erhaltungstherapie (4/5 Cotrimoxazol,1/5 Ciprofloxacin) 7-21 d. Es kam in keinem Fall zum septischem Schock oder Tod. Operative Maßnahmen waren nicht erforderlich. In allen Fällen erfolgte eine Beratung durch die Nephrologie Marburg (4/5 Fallkonferenz mit Bilddemonstration, 1/5 tel. Konsil), in 4/5 Fällen eine Verlegung und in 2/4 Fällen eine Rückverlegung zur Therapiefortführung.

Diskussion: Bei 29 Kindern mit HWI traten in $15 \%$ Komplikationen auf. Die Kooperation einer Kinderklinik der RV mit einem universitären Zentrum führte zu Fallkonferenzen, Verlegungen ohne Zwischenfälle. Alle Patienten zeigten eine Restitutio ad integrum. Geplant sind weitere Fortbildungen und ein Personalaustausch. Ein standardisiertes Vorgehen nach der geplanten AWMF Leitlinie (5) wäre sehr zu begrüßen.

\section{Literatur}

1. Zappel HF (2000) Harnwegsinfektionen. In: Speer, Gahr (Hrsg) Pädiatrie, 1. Aufl. Springer, in, $5772-776$

2. Elder JS (2016) Urinary Tract Infections, in Nelson Textbook of Pediatrics, 20. Aufl. Elesevier, , S 2556-2562

3. Gesetz über die Entgelte für voll- und teilstationäre Krankenhausleistungen (Krankenhausentgeltgesetz - KHEntgG) § 21 Übermittlung und Nutzung von Daten, in: https://www.gesetze-im-internet.de/khentgg/_21.html

4. Goldstein B (2005) Pediatr Crit Care Med 6(1):2-8

5. Beetz R et al Harnwegsinfektionen im Kindesalter - Diagnostik, Therapie und Prophylaxe (Angemeldetes Leitlinienvorhaben) Registernummer 166 - 004. https:// www.awmf.org/leitlinien/detail/anmeldung

\section{DGKJ-PO 41}

\section{Akutes Nierenversagen im Kindesalter - Ein Fallbeispiel}

Julian Norton Schäuble ${ }^{5}$ J. Schäuble ${ }^{1}$, M. C. Liebau ${ }^{1,2}$, K.-D. Nüsken', G. Cernaianu ${ }^{3}$, J. Salem ${ }^{4}$

${ }^{1}$ Klinik und Poliklinik für Kinder- und Jugendmedizin, Universitätsklinikum Köln; ${ }^{2}$ Zentrum für Molekulare Medizin, Universität zu Köln; ${ }^{3}$ Klinik und Poliklinik für Kinder- und Jugendmedizin, Schwerpunkt Kinderchirurgie, Universitätsklinikum Köln;'Klinik für Urologie, Uro-Onkologie, spezielle urologische und roboter-assistierte Chirurgie, Universitätsklinikum Köln; ${ }^{5}$ Uniklinik Köln, Pädiatrische Nephrologie, Köln, Deutschland

Hintergrund: Das akute Nierenversagen (ANV) ist mit einer Inzidenz von 4/1.000.000 ein seltenes Krankheitsbild im Kindes- und Jugendalter. Man unterscheidet zwischen prärenalem, renalem und postrenalem Nierenversagen. Dabei stellt das akute postrenale Nierenversagen im Kindesalter eine Rarität dar.

Kasuistik: Wir präsentieren den Fall eines 6-jährigen männlichen Patienten aus Angola, der vor 1,5 Jahren zur Behandlung einer chronischen Osteomyelitis des rechten Femurs nach Deutschland kam. Die Verlegung aus einer externen Klinik erfolgt bei oligurischem ANV und V.a. Sepsis. Die Sonographie der Nieren zeigte eine mäßige Erweiterung des Nierenbeckenkelchsystems beidseits ohne Nachweis von Megaureteren. Die weitere Bildgebung mittels MRT und CT zeigt eine beidseitige Pyonephrose mit beidseitiger Nephrolithiasis. Neben einer breiten antibiotischen Therapie erfolgte die Anlage von Doppel-J-Kathetern beidseits. Dies führte zu einer prompten Normalisierung der Retentionsparameter bei gutem Harnabfluss. Zwei Tage später entwickelt der Patient erneut ein ANV mit Anurie bei Obstruktion beider Doppel-J-Katheter. Es erfolgt der zystoskopische Wechsel auf Mono-J-Katheter beidseits mit erneuter Regredienz des ANV. Im Verlauf konnte ein Carbonatapatitstein geborgen werden.

Fazit: Dieser Fall zeigt exemplarisch, dass es trotz eines sonographisch relativ geringen Harnstaus sehr schwierig sein kann, ein akutes postrenales Nierenversagen auszuschließen.

\section{Literatur \\ 1. Dötsch J, Weber LT (2017) Nierenerkrankungen im Kindes und Jugendalter. Sprin- ger, Berlin, S 170-171}




\section{Mehrfachbehinderte Kinder/Varia}

\section{DGKJ-PO 42 \\ Weniger ist mehr - kalkulierte nicht-invasive Therapie bei chronisch kranken Kindern \\ Fatma Baysal', Katrin Braun'2, Caroline Stäbler ${ }^{2}$ \\ 'Klinikum Ludwigsburg, Klinik für Kinder- und Jugendmedizin, Ludwigsburg, Deutschland;'Klinikum Ludwigsburg, Kinderklinik, Ludwigsburg, Deutschland}

Einleitung: Kinder mit Mehrfachbehinderungen stellen eine pflegerische und ärztliche Herausforderung im stationären Alltag dar, da eine intensive Betreuung gewährleistet werden muss, um therapeutische Erfolge erzielen zu können. Hierbei ist eine enge Zusammenarbeit innerhalb des Teams und den Eltern erforderlich, um zu entscheiden, wie weit Therapiemöglichkeiten ausgeschöpft werden können und sollen. Vor invasiven Eingriffen bei chronisch kranken Kindern sollen erhöhte Risiken und die vermeintlichen Vorteile abgewogen werden. Ob nicht invasive Therapien, auch im Hinblick längerer Therapiedauer und Betreuung/stationären Aufenthalt, invasiven und konventionellen Maßnahmen vorzuziehen sind, muss individuell in Betracht gezogen und diskutiert werden.

Fallbericht: Wir präsentieren hier zwei Patienten, die bei uns fast zeitgleich, aufgrund einer Pneumonie und eine damit verbundenen respiratorischen Insuffizienz betreut werden wurden. Beide Patienten sind aufgrund Mehrfachbehinderungen und chronischer Lungenerkrankung mit einer Heimbeatmung bzw. zusätzlichen Sauerstoff zu Hause versorgt.

Pneumonien gestalten sich bi Kinder mit Mehrfachbehinderung häufig langwierig. Antibiotische Therapie bei bakterieller Infektion reicht meist allein für eine rasche Erholung nicht aus.

Es stellt sich in solchen Situationen die Frage, wie Kindern mit chronischen Lungenerkrankungen in einer akuten Infektion zusätzlich unterstützt werden können und wo eventuell die therapeutischen Grenzen erreicht werden.

Ziel war es, beide Patienten schonende pulmonale Rehabilitation zu erreichen, und die Risiken einer invasiven Beatmung zu umgehen.

Das Sekretmanagement stellt häufig ein wesentliches Problem dar, welches die Oxygenierung beeinträchtigt. Durch konservativen Maßnahmen wie Inhalationstherapien, Atemgymnastik und Cough und die Möglichkeit der nicht-invasiven Beatmung mittels High Flow, trugen wesentlich zur Besserung der pulmonalen Symptomatik bei.

Schlussfolgerung: Neben den invasiven und medikamentösen Therapieansätze sind konservative Therapieansätze unumgänglich. Vor allem ist es wichtig, die intensive Atemgymnastik im Rahmen des stationären Aufenthaltes konsequent zu etablieren und fortzuführen. Unterstützende devices und deren Anleitung und Anwendung sind hier von großer Bedeutung. Der Beatmungsmodus chronisch kranker Patienten mit Hinblick auf ihre Grunderkrankung muss gut durchdacht werden. Invasiv beatmete Patienten ermöglichen uns eine weitreichende Eingriffs- und Steuerungsmöglichkeit.

Allerdings birgt die invasive Beatmung bei den meist muskulär hypotonen Patienten auch die Gefahr einer Langzeitbeatmung bzw. Respiratorabhängigkeit in sich. Eine intensive nicht-invasive Beatmung kann eine wirkungsvolle Alternative darstellen.

Gemeinsam mit den Eltern müssen Vor- und Nachteile für das betroffene Kind und langfristig für die Familie diskutiert werden, um eine möglichst optimale, in die individuelle Situation passende therapeutische Lösung zu finden.

\section{DGKJ-PO 43 \\ Speckle Tracking Stressechokardiographie bei Kindern: Inter- und Intraobserver-Reproduzierbarkeit und der Einfluss der echokardiographischen Bildqualität}

Lucia Wilke' , Kai O. Hensel', Markus Roskopf ${ }^{2}$, Andreas Jenke ${ }^{2}$, Francisca Abellan-Schneyder ${ }^{2}$, Andreas Heusch ${ }^{2}$

'Universität Witten/Herdecke, Witten, Deutschland;'niversität Witten/ Herdecke, Zentrum für Kinder und Jugendmedizin, Witten, Deutschland

Speckle Tracking Echokardiographie (STE) erhält als Methode zur Quantifizierung myokardialer Funktion zunehmend Einzug in die Diagnostik der kardialen Leistungsfähigkeit mittels Stressechokardiographie. Auf diese Weise können regionale Wandbewegungsstörungen erkannt werden, die unter Ruhebedingen möglicherweise unentdeckt blieben. In der Pädiatrie hat sich die Kombination der beiden Methoden allerdings noch nicht etabliert, weshalb ihre Reproduzierbarkeit und der Einfluss der Bildqualität darauf für den Einsatz bei Kindern noch nicht untersucht sind.

Im Rahmen dieser Studie wurden 127 herzgesunde, normal entwickelte Kinder (Durchschnittsalter 13,4 $\pm 3,0$ Jahre, 50,4 \% weiblich) in Ruhe und unter Belastung mittels Fahrrad-Ergometrie echokardiographiert. Zur STE Analyse wurden zweidimensionale B-Mode-Bilder aufgezeichnet und anschließend mit einer Quantifizierungssoftware analysiert. Dabei wurde segmentaler und globaler linksventrikulärer Strain und die Strain rate sowohl in Ruhe als auch unter Belastung bestimmt und die Messungen auf ihre Inter- und Intraobserver-Reproduzierbarkeit untersucht. Eine Einteilung der Bilder an Hand der prozentualen Myokardsichtbarkeit in drei Qualitätskategorien ermöglichte den Vergleich der Messabweichungen zwischen den drei Gruppen. Die Darstellung der Messunterschiede erfolgte in Bland-Altman-Diagrammen. Zur Veranschaulichung der Variabilität unter Berücksichtigung der Bildqualität wurden Streudiagramme verwendet.

LV globaler Strain und Strain rate waren mit schmalen limits of agreement in allen Belastungsstufen sehr gut reproduzierbar. Für Strain-rate-Werte zwischen -1,5 und -3,0 s-1 nahmen die Messabweichungen leicht $\mathrm{zu}$. Interessanterweise hatte die Bildqualität keinen relevanten Einfluss auf die Reproduzierbarkeit der Messungen. Die Variabilität der Messungen war für Bilder mit einwandfreier, mittelmäßiger und suboptimaler Bildqualität gleich gering.

Mittels STE erhobene Strain- und Strain-rate-Messungen bei Kindern sind unter Fahrrad-Ergometrie-Belastungsechokardiographie gut durchführbar und sehr gut reproduzierbar. Die Bildqualität der echokardiographischen Untersuchung scheint dies kaum zu beeinflussen. Da der Bearbeitungssoftware aber auch bei unzureichender Myokardsichtbarkeit ein scheinbar einwandfreies Tracking mit physiologischen Messwerten gelingt, müssen diese myokardialen Deformationsparameter mit Vorsicht gedeutet werden. In zukünftigen echokardiographischen Studien sollte die Bildqualität explizit deklariert und in die Interpretation einbezogen werden.

\section{DGKJ-PO 44}

\section{Exomsequenzierung: Symptombezogene Auswertung mittels Human Phenotype Ontology (HPO)}

Maren Wenzel', Hans-Martin Büttel', Alina Henn'2, Marius Kuhn', Birgit Zirn²

'genetikum Neu-Ulm, Genetische Beratung, Neu-Ulm,

Deutschland;'2genetikum Stuttgart, Genetische Beratung, Stuttgart, Deutschland

Die Exomsequenzierung hat in der Diagnostik von Kindern mit Entwicklungsstörungen einen hohen Stellenwert - vor allem, wenn zusätzliche Symptome wie Wachstumsstörungen, angeborene Fehlbildungen, eine Epilepsie oder Dysmorphien vorliegen. Alle proteincodierenden Abschnitte des Genoms des Patienten werden computergestützt ausgelesen. Über eine symptombezogene Auswertung gelingt in vielen Fällen der Nachweis der ursächlichen Veränderung. Dazu werden die Auffälligkeiten des Kindes gemäß der „Human Phenotype Ontology“ (HPO) bezeichnet und codiert und dann systematisch mit internationalen Datenbanken wie 
z. B. OMIM oder Orphanet abgeglichen. Im Vortrag werden fünf klinische Fälle aus unserer Sprechstunde präsentiert. Dabei wird insbesondere auf die klinische Symptomatik, die HPO-Codierung und das Procedere zur Auswertung bis hin zur Diagnosestellung eingegangen.

\section{DGKJ-PO 45}

Effect of sebelipase alfa on survival to 3 years of age and liver function in infants with rapidly progressive lysosomal acid lipase deficiency: Results from two studies

Simon A. Jones', Suresh Vijay², Simona Fecarotta ${ }^{3}$, Arunabha Gosh', Kerstin Allen ${ }^{4}$, Mark Friedman $^{4}$, Florian Abel ${ }^{4}$

'Manchester University Hospitals NHS Foundation Trust St Mary's Hospital, n. a., Manchester, United Kingdom;'Birmingham Children's Hospital, n. a., Birmingham, United Kingdom; ${ }^{3}$ Frederico II University, n. a., Naples, Italy; ${ }^{4}$ Alexion Pharmaceuticals, Inc., n. a., New Haven, CT, United States

Two studies (VITAL/CL08) are evaluating the safety and efficacy of enzyme replacement with sebelipase alfa (SA) in infants with rapidly progressive lysosomal acid lipase deficiency (LAL-D). Nineteen infants were enrolled (VITAL, $n=9$; CL08, $n=10$ ). Median age at treatment initiation for VITAL/CL08 was 3.0/2.8 months. As of August 2017, 13 patients continue on study or completed the study; 6 have died. Five are $\geq 3$ years old (all in VITAL) and have been receiving SA for 228-263 weeks. All patients experienced $\geq 1$ serious adverse event (SAE). Seven ( $37 \%$ ) had treatment-related SAEs; of these, 5 had infusion-associated reactions (IARs), including 1 patient with an IAR who also had pyrexia. Among patients who did not have an IAR, 1 developed anti-drug antibodies (ADAs), and 1 had pyrexia, pallor, chills, and tachycardia. All SAEs resolved. None discontinued treatment because of tolerability/IARs. Of 17 patients tested for ADAs, 10 had detectable titers; of these, 8 developed neutralizing antibodies, with no apparent effects on safety/efficacy. Median serum alanine aminotransferase levels, 145.0 U/L at baseline (BL, $n=9$ ) in VITAL, decreased by $29.6 \%$ at week $48(n=4), 7.4 \%$ at week $96(n=5)$, and $12.5 \%$ at week $144(n=5)$; $37 \mathrm{U} / \mathrm{L}$ at BL $(n=9)$ in CL08, they showed no change at week $48(n=7)$, decreased by $55.2 \%$ at week $96(n=5)$, and decreased by $90.3 \%$ at week $144(n=2)$. Serum hemoglobin and albumin levels also improved. Median weight centile, $3.1(n=8)$ at BL, increased by $7.5(n=4)$ at week $48,21.8$ $(n=5)$ at week 96, and $14.0(n=5)$ at week 144 in VITAL; and $0.2(n=9)$ at BL in CL08, increased by $27.2(n=7)$ at week $48,45.5(n=5)$ at week 96 , and $61.7(n=3)$ at week 144 .

In conclusion, $\mathrm{SA}$ is associated with prolonged survival, favorable tolerability, and sustained improvements in disease activity parameters in in fants with LAL-D.

\section{DGKJ-PO 46}

Patients with LC-FAOD have poor clinical outcomes despite newborn screening

Deborah Marsden', Camille Bedrosian', Jerry Vockley²

'Ultragenyx Pharmaceutical Inc., Clinical Development, Novato, United States; ${ }^{2}$ University of Pittsburgh, Pediatric Medical Genetics, Pittsburgh, PA, United States

Background: Long-chain fatty acid oxidation disorders (LC-FAOD) are rare, genetic disorders characterized by impaired fatty acid metabolism. Patients (pts) with LC-FAOD experience frequent hospitalizations and high mortality due to serious disease consequences including hypoglycemia, cardiomyopathy, and rhabdomyolysis. Current disease management includes low-fat/high-carbohydrate diet, medium chain triglycerides (MCT), and avoidance of fasting. Pts are diagnosed by newborn screening (NBS) in several countries, and otherwise are diagnosed symptomatically. Methods: To evaluate the difference in clinical outcomes in pts diagnosed by NBS compared with pts diagnosed symptomatically, we conducted PubMed electronic searches to identify publications (in English) relating to mortality rates in pts with LC-FAOD.
Results: In a retrospective review of 75 pts with LC-FAOD in Germany, Switzerland, Austria and the Netherlands (Spiekerkoetter 2009), 32 pts were detected by NBS. No mortalities were reported in screened pts with very long-chain acyl-CoA dehydrogenase (VLCAD) or long-chain 3-hydroxy-acyl-CoA dehydrogenase (LCHAD) deficiency. Of 3 screened pts diagnosed with trifunctional protein (TFP) deficiency, two pts died at 3 and 8 days of age. Two of three screened pts diagnosed with carnitine palmitoyl transferase-2 (CPT-2) deficiency died at 6 and 7 months of age. Among pts diagnosed symptomatically, 2/10 with VLCAD deficiency, 3/13 with LCHAD deficiency, and 3/4 with TFP deficiency died from 2 days to 4 yrs of age.

A study in Europe of 50 pts with LCHAD deficiency diagnosed without NBS reported a mortality rate of $38 \%$ (den Boer 2002). 14 pts died before diagnosis and five died within 3 months of diagnosis. Seven pts were diagnosed neonatally. Eight pts died within 8 wks of presentation due to cardiac complications. Acute hypoglycemia accounted for $38 \%$ of deaths, and $36 \%$ of children with chronic nonspecific symptoms died.

In a study of 21 pts with TFP deficiency in the UK and the Netherlands (den Boer 2003), 10 presented neonatally. 9 pts had rapidly progressive clinical deterioration, including hypoglycemia. Twelve pts had nonspecific chronic symptoms, including hypotonia and cardiomyopathy. Sixteen pts died, mostly due to cardiac involvement. Two pts diagnosed prenatally died despite treatment.

A retrospective study in France analyzed 187 children with FAOD diagnosed $<6$ yrs of age without NBS (Baruteau 2012). Mortality rates were $>60 \%$ for pts with LCHAD, VLCAD, and CPT-2 deficiency.

A retrospective chart review of 37 pts with VLCAD deficiency in Saudi Arabia found that 23 pts died before the age of 2 yrs, despite NBS and early treatment, including MCT supplementation (Obaid 2018).

Conclusions: Although outcomes have improved with NBS, major medical events still occur despite early diagnosis and disease management. These literature review results underscore the need for urgent early intervention and new treatment options for pts with LC-F

\section{DGKJ-PO 47}

\section{Ergebnisse eines 6 min-Laufs bei adipösen sowie} normalgewichtigen Kindern und Jugendlichen an der Klinik Schönsicht Berchtesgaden

Richard Eyermann ${ }^{1,2}$

'Dr. Eyermann München, Kinder- und Jugendmedizin, Kinderkardiologie, Sportmedizin, München, Deutschland; ${ }^{2 K l i n i k}$ Schönsicht Berchtesgaden, Rehabilitation für Kinder und Jugendliche, AHB, Kind-Mutter/VaterRehabilitation, München, Deutschland

Problemstellung: Leistungsdiagnosen dienen zur Einordnung des aktuellen Leistungszustandes. Dies ist besonders wichtig an Kliniken, da hier anhand von Anfangs- und Enddiagnosen Schlüsse über den Erfolg des Klinikaufenthaltes gezogen werden. Bestandteil der Diagnose ist oftmals auch ein Sporttest.

Methodik: In der vorliegenden Studie wurden Daten (Januar 2017 bis Mai 2018, $n=298$ ) einer Klinik für Rehabilitation von Kindern und Jugendlichen, AHB und Kinder-Mutter-/Vater-Rehabilitation zum 6 min-Lauf, der Bestandteil der Leistungsdiagnose ist, ausgewertet. Dabei wurde die Anfangsleistung mit der Endlaufleistung verglichen, der BMI mit der Anfangs- und Endlaufleistung korreliert, der Retest bezüglich der Reliabilität ausgewertet, die sportliche Aktivität des vorangegangenen Jahres mit der Anfangslaufleistung korreliert, sowie die Standardisierung des 6 minLaufs beschrieben.

Ergebnisse und Diskussion: Die Ergebnisse konnten eine Steigerung der Laufleistung mit einer durchschnittlichen Verbesserung von $81 \mathrm{~m}$ bei den Jungen bzw. $108 \mathrm{~m}$ bei den Mädchen belegen. Während eine hohe Korrelation zwischen Retest und Laufleistung festgestellt werden konnte, besteht nur eine geringe Korrelation zwischen BMI und Laufleistung sowie zwischen der sportlichen Aktivität im vorangegangenen Jahr und der Anfangslaufleistung. Bezüglich der Standardisierung des 6 min-Laufs konnte 
ein hoher Durchführungsstandard festgestellt werden, während die Teststandards an sich vereinzelt Mängel aufwiesen.

Konklusion: Für die Praxis kann geschlussfolgert werden, dass der 6 minLauf durchaus ein Mittel zur Leistungsdiagnose darstellt. Dennoch ist zu berücksichtigen, dass er oftmals zur Analyse der aeroben Ausdauerleistung durchgeführt wird. Dies kann aber nur unter Berücksichtigung der Gütekriterien erfolgen, die neben der Standardisierung auch die Validität umfassen. Diese kann jedoch erst anhand eines weiteren Kriteriums, neben der Laufleistung, wie beispielsweise der Schwimmleistung, festgestellt werden und erfordert folglich zusätzliche Daten zur Auswertung. L. Heinrichs: Ergebnisse eines 6 min-Laufs bei adipösen sowie normalgewichtigen Kindern und Jugendlichen an der Klinik Schönsicht Berchtesgaden. Masterarbeit Sportwissenschaft, Universität Mainz.

\section{DGKJ-PO 48 Auffälligkeiten des Ernährungsverhaltens von an Cystischer Fibrose erkrankten Kindern und deren Einfluss auf das familiäre Zusammenleben}

Marcella Berndt-Müller', Isolde Krug', Michael Barth', Andrea Heinzmann" ${ }^{2}$ 'Universitätsklinikum Freiburg, Zentrum für Kinder- und Jungendmedizin pädiatrische Pneumologie, Allergologie und Mukoviszidose, Freiburg i. Br., Deutschland; ${ }^{2}$ Universitätsklinikum Freiburg, Zentrum für Kinderund Jugendmedizin, pädiatrische Pneumologie, Allergologie und Mukoviszidose, Freiburg, Deutschland

Fragestellung: Ein wesentlicher Bestandteil in der Therapie der CF ist die Ernährungstherapie. Ein stabiler Ernährungszustand ist entscheidend für den gesundheitlichen Verlauf eines an CF erkrankten Kindes. Dabei stellt die empfohlene Aufnahme der bis zu 50 \% erhöhten Kalorienzufuhr im Vergleich zu gesunden Gleichaltrigen für die Betroffenen und deren $\mathrm{Fa}-$ milien eine erhebliche Herausforderung dar. Viele CF kranke Kinder entwickeln schon frühzeitig Ernährungsprobleme und Schwierigkeiten im Essverhalten. Die bisherigen Daten zur Auswirkung dieser Herausforderungen auf den familiären Alltag sind limitiert. Im Rahmen dieser Studie werden elterliche Strategien zum Umgang mit der chronischen Erkrankung unter besonderer Berücksichtigung der Ernährungssituation betrachtet

Material und Methode: Es handelt sich um eine gemischte Querschnittsstudie mit Eltern von CF kranken Kindern im Alter von 2 bis 14 Jahren, die sich in der medizinischen Behandlung an einem CF-Zentrum (Universitätskinderklinik Freiburg, Deutschland) befinden. Es nahmen 16 von 32 Familien an der Studie teil. Mithilfe zweier Fragebögen und individuellen Interviews wurden die Ernährungssituation und das Verhalten der Kinder während der Mahlzeiten erhoben und darüber hinaus die familiäre Belastung und der Umgang mit der Krankheitssituation im alltäglichen Leben herausgestellt. Ein Fragebogen diente zur Erfassung des familiären Alltags von Familien mit einem chronisch kranken Kind; hier wurde der Family Management Measure von Knafl et al. 2013 verwendet. Mit einem weiteren Fragebogen wurden ernährungsbedingte Auffälligkeiten im kindlichen Verhalten sowie elterliche Reaktionsweisen darauf erhoben (Behavioral Pediatrics Feeding Assessment nach Crist et al. (2001)).

Ergebnisse: 6 der 16 Familien berichten über ein bestehendes problematisches Ernährungs- und Essverhalten ihres an CF erkrankten Kindes, 4 andere berichten von ernsthaften Ernährungsproblemen in der Vergangenheit. Eltern von Kindern mit problematischem Essverhalten schildern häufiger Schwierigkeiten im familiären Alltag, eine höhere emotionale Belastung und geringere Fähigkeiten im Umgang mit der chronischen Erkrankung. Probleme im Essverhalten korrelieren in hohem Masse mit negativen Zukunftserwartungen für das CF kranke Kind.

Diskussion: Zu beachten ist, dass die Einschätzung der ernährungsbedingten Probleme der betroffenen Kinder auf den Angaben der Eltern beruht. Eine objektive Erfassung des Ernährungszustandes ist hierbei noch nicht berücksichtigt und wird im Anschluss anhand von Perzentilenverläufen erhoben.

Schlussfolgerung: Ernährungsbedingte Schwierigkeiten bei einem an CF erkrankten Kind werden von Eltern als Quelle einer hohen psychischen
Belastung beschrieben. Chronische Ernährungsprobleme gehen mit einer geringeren Zufriedenheit im täglichen Familienleben einher und mit weniger Sicherheit im Umgang mit der chronischen Krankheit. Eine umfassende Ernährungs- und Verhaltensberatung zur Unterstützung sollte angeboten werden.

\section{DGKJ-PO 49}

Auswirkungen eines 3-wöchigen Interventionsprogramms auf die Körperzusammensetzung bei adipösen Kindern und Jugendlichen gemessen mit bioelektrischer Impedanzanalyse (BIA) an der Klinik Schönsicht

\section{Richard Eyermann ${ }^{1,2}$}

'Dr. Eyermann München, Kinder- und Jugendmedizin, Kinderkardiologie, Sportmedizin, München, Deutschland;'Klinik Schönsicht Berchtesgaden), Rehabilitation für Kinder und Jugendliche, AHB, Kind-Mutter VaterRehabilitation, München, Deutschland

Hintergrund: Adipositas nach WHO eines der größten Gesundheitsprobleme des 21. Jh.

Ziel war die Wirksamkeit der sportlichen Intervention in der Reha zu überprüfen, ob diese zu der gewünschten Gewichtsreduktion durch Verlust von Körperfett führt u. ob in einem Interventionszeitraum von 3 Wo. schon sign. Ergebnisse erzielt werden können.

Methodik: 34 Kinder u. Jugendliche mit Adipositas stationär in Reha multimodal behandelt, 20 w. u. 14 m., im Alter von 10-17 Jahren, BMI $34,6 \mathrm{~kg} /$ $\mathrm{m}^{2}$ (MW) $\pm 6,9$ (SD) bzw. 33,3 kg/m² (MW) \pm 7,4 (SD).

Einschlußkriterien: für mindestens 4 Wo. Patient, Diagnose Adipositas, ärztl. Sporterlaubnis, zwischen 10-18 Jahren. Ausschlusskriterien: Medikamente, die Flüssigkeitshaushalt beeinflussen, Infekt (akut während letzter 14 Tage), metallische Implantate (OP-induziert), Schwangerschaft, Herzschrittmacher, Defibrillator.

Messungen mit 2 BIA-Geräten, MALTRON Körperfett-Analysegerät BF906 u. BIA-Gerät „DENNER Systemtechnik“.

Daten sowohl deskriptiv als auch inferenzstatistisch ausgewertet (Statistikprogramm IBM SPSS 21).

Ergebnisse u. Diskussion: Studie untersuchte kurzfristige Auswirkungen von körperlicher Bewegung auf die Körperzusammensetzung. Verwendetes Studiendesign ist durchaus gut gewählt u. bietet gute Grundlage um die Fragen zu beantworten. Allerdings bleiben hier die Langzeitwirkungen außen vor, welche gerade im Bereich der kindlichen u. jugendlichen Adipositas von großer Bedeutung sind, wie Folgeerkrankungen. Trotz des eher geringen Zeitraums zeigt die Studie die Effektivität solcher Interventionsprogramme u. konnte dem Ziel der Gewichtsreduktion durch Körperfettabnahme gerecht werden.

- Körpergewicht: Der MW lag beim Prae-Test bei 89,74 $\pm 18,46 \mathrm{~kg}$ u. bei der Messung nach 3 Wo. bei 86,05 $\pm 17,58 \mathrm{~kg}$ bei den Mädchen, bei den Jungen MW 97,34 $\pm 35,81 \mathrm{~kg}$ bzw. $92,21 \pm 33,33 \mathrm{~kg}$.

- Körperfett: Fettanteil sank von 39,13 $\pm 3,58 \%$ auf 37,34 $\pm 3,48 \%$ bei den Mädchen, bei den Jungen von 33,61 $\pm 9,38 \%$ auf 30,89 $\pm 10,18 \%$.

- Muskelmasse: Zunahme um 0,29 \% bei den Mädchen bzw. 0,33 \% bei den Jungen.

- Körperwasser: Zunahme bei Mädchen u. Jungen.

- Body Cell Mass: Steigerung um 2,73 \% bei den Mädchen u. 2,56 \% bei den Jungen.

- Resistanz(R) u. Reaktanz(Xc): Veränderungen waren bei keinem der beiden Geschlechter signifikant.

- Phasenwinkel: Veränderung nicht signifikant.

Der ebenfalls untersuchte Gerätevergleich zeigt auf, dass die verwendeten BIA-Geräte gleich messen. Sie haben dasselbe Funktionsprinzip. Für zukünftige Studien besteht hier die Option, weitere Meßgeräte mit einzubeziehen $u$. diese untereinander zu vergleichen, z. B. sind Vergleiche zwischen BIA, MRT u. DEXA möglich, zudem können diese mit „einfachen u. kostengünstigen “ Varianten, wie der Hautfaltenmessung verglichen werden.

Konklusion: Angewandte sportliche Interventionen in der Reha erreichen die positiven u. erwünschten Wirkungen $u$. sollten als wirksame Maßnah- 
me gegen die Volkskrankheit Adipositas beibehalten u. etabliert werden. Studie trägt zu neuen Erkenntnissen bei Adipositas bei.

M. Kaestner: Auswirkungen eines 3-wöchigen Interventionsprogramms auf die Körperzusammensetzung bei adipösen Kindern und Jugendlichen gemessen mit bioelektrischer Impedanzanalyse (BIA) an der Klinik Schönsicht.

Masterarbeit Sportwissenschaft, TU Chemnitz

\section{DGKJ-PO 50}

Bioäquivalenz von auf Lebensmittel gestreutem und direkt verabreichtem Hydrocortison-Granulat mit Geschmacksmaskierung und altersgerechter Dosierung

Eleni Daniel', Dena Digweed ', Jo Quirke'2, Bernard Voet ${ }^{2}$, Hanna Bendfeldt', Madhu Davies', Richard John M Ross'

'University of Sheffield, Oncology and Metabolism, Sheffield, UK;'²Diurnal Limited, Development, Cardiff, UK; ' Diurnal Limited, Medical Affairs, Cardiff, UK

Hintergrund: Studien zu individuell hergestellten Hydrocortison-Rezepturen für Kinder zeigen, dass bis zu $25 \%$ der in den Apotheken und $50 \%$ durch Eltern hergestellten Hydrocortisonzubereitungen nicht den Spezifikationen des Europäischen Arzneibuches entsprechen. Alkindi ${ }^{\circledast}$ (Diurnal Ltd., UK), ein Hydrocortison-Granulat in kindgerechten Dosierungen, ist mit einer Geschmacksmaskierung versehen und lässt sich leicht verabreichen. So werden adäquate Cortisolspiegel in Neugeborenen, Säuglingen und Kindern erreicht.

Material und Methoden: In dieser randomisierten, Einzeldosis-, 3-Perioden-Crossover-Studie in 18 Dexamethason behandelten, gesunden Männern sollte die Bioverfügbarkeit von auf Soft Food gestreutem und direkt auf die Zunge verabreichtem Hydrocortison-Granulat verglichen werden. In jeder Periode erhielten die Teilnehmer nüchtern 5 mg Alkindi mit einer von 3 Verabreichungsmethoden: direkt auf den Zungenrücken, auf $5 \mathrm{ml}$ Soft Food (Apfelmus) und auf $5 \mathrm{ml}$ Joghurt. Cortisol im Serum wurde mittels LCMS/MS vor und bis zu $12 \mathrm{~h}$ nach der Verabreichung gemessen (ClinicalTrials.gov NCT03178214).

Ergebnisse: Die mittleren Cortisol Cmax und AUC0-inf Werte für die direkte Verabreichung, Granulat auf Joghurt und Granulat auf Soft Food waren: Cmax 428, 426, $427 \mathrm{nmol} / \mathrm{l} \& \mathrm{AUC} 0$-inf 859, 886, $844 \mathrm{~h}{ }^{\star} \mathrm{nmol} / \mathrm{l}$. Die 90 \% Konfidenzintervalle (CI) für das Verhältnis von Cmax \& AUC0-inf nach Verabreichung mit Soft Food oder Joghurt zur direkten Verabreichung lagen deutlich innerhalb der 80-125\%-Grenze, was die Bioäquivalenz bestätigt. Der Median Tmax betrug $0,63 \mathrm{~h}$ für direkt verabreichtes Granulat, $0,75 \mathrm{~h}$ für Granulat auf Soft Food und $0,75 \mathrm{~h}$ für Granulat auf Joghurt. Während der Studie traten keine unerwünschten Ereignisse auf. Schlußfolgerung: Alkindi ${ }^{\oplus}$ Hydrocortison Granulat, das als Streusel auf Apfelmus oder Joghurt verabreicht wird, ist bioäquivalent zu direkt auf die Zunge gestreutem Granulat. Betreuer, Eltern und Patienten können Alkindi ${ }^{\circledR}$ entweder direkt als Trockengranulat verabreichen oder auf Soft Food oder Joghurt streuen.

\section{DGKJ-PO 51}

\section{The MPS VII Disease Monitoring Program (DMP) is a novel, longitudinal, cohort program with rigor beyond a traditional registry}

Deborah Marsden, Robert Hostutler, Tricia Cimms, Kenneth J Sansone, Marcel van Kuijck

Ultragenyx Pharmaceutical Inc., Medical Affairs, Novato, CA, United States

MPS VII is an ultra-rare, autosomal recessive, heterogeneous, debilitating, progressive lysosomal disease caused by beta-glucuronidase (GUSB) enzyme deficiency. Vestronidase alfa (recombinant human GUSB) is an approved enzyme replacement therapy for MPS VII. Given the rarity of MPS VII, there is a need to better understand disease progression and evaluate long-term treatment with vestronidase alfa.
The MPS VII Disease Monitoring Program (DMP) is a multicenter program collecting long-term real-world data from patients with MPS VII, regardless of treatment status. To overcome the common registry challenge of missing data, the DMP is designed to provide standardized, consistent, focused outcomes data. Site selection is based on MPS expertise and location, to maximize enrollment. To ensure retention, appropriate site compensation and travel assistance are provided.

The DMP aims to enroll approximately 35 patients with MPS VII worldwide. Diagnosis will be confirmed by GUSB enzyme assay and mutation analysis. Key assessments include demographics; clinical history; clinical presentation; cognition, mobility, skeletal disease, and pulmonary function; patient/caregiver-reported outcomes and health-related quality of life; and long-term vestronidase alfa safety and effectiveness. Assessments occur at baseline, every 6 months for the first year (for patients $\geq 5$ years) or first 2 years (for patients $<5$ years), then annually thereafter for up to 10 years. Data will be source verified based on GCP standards.

By standardizing assessments, monitoring with source document verification, and focusing on patient retention, the DMP will address the shortcomings of traditional registries and provide a comprehensive dataset offering a greater understanding of MPS VII disease course.

Disclosures:

- Deborah Marsden, Robert Hostutler, Tricia Cimms, and Marcel van Kuijck are employees and shareholders of Ultragenyx Pharmaceutical Inc.

- Ken Sansone has consulted for and is a shareholder of Ultragenyx Pharmaceutical Inc. 


\section{Neonatologie und Intensivmedizin/Varia 1}

\section{DGKJ-PO 52 \\ Hydrops fetalis mit Hexadaktylie - Diagnose eines Noonan- Syndroms durch Exom-Diagnostik}

Rebecca Nathalie Schäfer', Annemarie Bösch², Felix Boschann 3, Jana Hinkel', Denise Horn ${ }^{3}$, Christoph Bührer', Christof Dame ${ }^{1}$

${ }^{1}$ Charité - Universitätsmedizin Berlin, Klinik für Neonatologie, Berlin, Deutschland, ${ }^{2}$ Charité - Universitätsmedizin Berlin, Berliner Centrum für Seltene Erkrankungen, Charité, Berlin; ${ }^{3}$ Charité - Universitätsmedizin Berlin, Institut für Medizinische Genetik und Humangenetik, Berlin

Fallbericht: Wir berichten von einem weiblichen Zwillings-Frühgeborenen (dichorial-diamniot; $35+4$ SSW) gesunder, nicht-konsanguiner nigerianischer Eltern (33-Jährige G5/P4). Pränatal Hydrops fetalis; unauffälliger 2. Zwilling.

Erstversorgung: Primäre Intubation bei ausgeprägtem Hydrothorax, umgehende Pleuradrainage bds., HFO-Beatmung. Apgar 2/4/9. Labor: Leichte Thrombozytopenie (147/nl), plasmatische Gerinnungsstörung, keine Anämie, übrige Labordiagnostik unauffällig. Phänotyp: Breite Nasenwurzel, tiefstehende Ohren, hoher Gaumen, persistierende obere Hohlvene, postaxiale Hexadaktylie bds. Im Verlauf suprasystemische pulmonale Hypertension, Beatmung mit inhalativem NO. Extubation am 10. Lebenstag. Unter Ernährung chylöse Pleuraergüsse, Persistenz unter fettfreier Ernährung und Therapie mit Octreotid über 10 Tage. Sonographisch zunehmende biventrikuläre kardiale Hypertrophie mit Herzinsuffizienz bei pulmonaler Hypertonie, pulsatiler Fluss in der hypoplastischen Vena portae mit großem Pooling-Gefäß zwischen Umbilikalvene und Vena cava inferior, Thrombose in der V. portae. Im MRT dilatierte Lymphabflussbahnen zervical, thorakal und abdominal. Zunehmende respiratorische Insuffizienz trotz wieder begonnener Beatmung bei therapie-refraktärem Chylothorax, dann Pneumonie. Im Konsens mit den Eltern Therapiebegrenzung. Das Kind verstarb am 48. Lebenstag im Beisein der Eltern.

Schlussfolgerung: Zur Klärung des Krankheitsbildes wurde eine ExomDiagnostik der Patientin mit anschließender Segregationsanalyse der Eltern und des Zwillings-Geschwisterkindes im Rahmen des TRANSLATE-NAMSE Projekts durchgeführt. Das Versorgungsprojekt, welches aus dem Innovationsfonds des Gemeinsamen Bundesausschusses gefördert wird, widmet sich der Versorgung von Patient*innen mit seltenen Erkrankungen. In der Diagnostik fand sich eine heterozygote Mutation (Chr. 2 rs40651; c508A>G, Lys170G; CM086966 der Human Genome Mutation Database) im SOS1 Gen (Son of Sevenless), so dass ein Noonan-Syndrom (OMIM 163950) als Ursache des Krankheitsbildes vorliegt.

Das SOS1 Gen kodiert für GEF (Guanine Nucleotide Exchange Factor), welches RAS in die aktive Form konvertiert und so den RAS-MAPK-Signalweg stimuliert. Der Fallbericht belegt den Stellenwert der Next Generation Sequencing Analyse zur zügigen Diagnostik unklarer komplexer Krankheitsbilder in der Neonatologie.

\section{DGKJ-PO 53}

\section{Rizinsamenintoxikation}

Cornelia Vogel, Fabian Kaßberger

ALB FILS KLINIKEN, Klinik für Kinder- und Jugendmedizin, Göppingen, Deutschland

Hintergrund: Ricinus communis ist eine in Deutschland verbreitete Zierpflanze, deren Samen (Kastorbohnen) das hochpotente Zellgift Rizin enthalten. Kastorbohnen sind frei im Handel verfügbar. Die letale orale Dosis wird mit 1-20 mg/kg KG angegeben, wobei der Rizingehalt der Samen starken Schwankungen unterliegt. Die Varianz der Toxizität in der Literatur reicht von einem letalen Ausgang nach Ingestion von 2 Kastorbohnen bis zu einer überlebten Dosis von insgesamt 60 Kastorbohnen.

Fallbericht: Eine 17-jährige Patientin wurde durch den Notarzt auf unsere Intensivstation eingewiesen, nachdem sie $5 \mathrm{~h}$ vor Aufnahme circa 40
Rizinsamen in suizidaler Absicht im Rahmen einer schweren Depression eingenommen hatte.

Klinisch präsentierte sich die Patientin mit starker Übelkeit und Erbrechen sowie Tachykardie; im weiteren Verlauf auch mit einem Anstieg der Körperkerntemperatur auf max. $39^{\circ} \mathrm{C}$, einem erworbenen long-QT-Syndrom und einer arteriellen Hypotension. Nach Aufnahme erfolgten eine Notfallgastroskopie sowie die wiederholte Gabe von medizinischer Kohle und Glaubersalz zur Minimierung der aufgenommenen Toxinmenge. Bei Aufnahme zeigten sich keine laborchemischen Auffälligkeiten, im Verlauf kam es zu einem Anstieg der LDH, CK und des CRP im Rahmen einer Rhabdomyolyse sowie eines ASAT-Anstiegs und INR-Abfalls bei hepatotoxischer Wirkung mit Maximum am 5. Tag post ingestionem.

Unter symptomatischer Therapie mit Infusionstherapie, Elektrolytausgleich, Nahrungskarenz und physikalischer Kühlung wurde eine schrittweise Restitutio ad integrum erreicht, sodass die Patientin nach 7 Tagen in eine psychiatrische Klinik verlegt werden konnte.

Fazit: Kürzlich ist Rizin durch einen geplanten bioterroristischen Angriff in den Fokus der Öffentlichkeit gelangt. Daten über den klinischen Verlauf von Intoxikation sind daher von besonderem Interesse. Unsere Patientin hat sich trotz der potenziell letalen Dosis komplett erholt.

\section{Literatur}

1. Musshoff F, Madea B (2009) Ricin poisoning and forensic toxicology. Drug Test Anal 1:84-91

2. Challoner KR, McCarron MM (1990) Castor bean intoxication: review of reported cases. Ann Emerg Med 19:1177-1183

3. Müller SM, Mehlig A, Zimmerli W Anleitung zum

\section{DGKJ-PO 54}

\section{Intranasale Muttermilchapplikation bei sehr kleinen} Frühgeborenen mit intraventrikulärer Hirnblutung

\section{Franz Mierke, Lars Mense, Mario Rüdiger, Barbara Seipolt}

Universitätsklinikum Carl Gustav Carus Kinderklinik, Neonatologie, Dresden, Deutschland

Hintergrund: Tierexperimentelle Daten zeigen positive Effekte einer intranasalen Applikation von Stammzellen und Wachstumsfaktoren auf den Verlauf zerebraler Schädigungen bei Neugeborenen. Basierend auf ersten Erfahrungen mit der intranasalen Applikation von Muttermilch bei Frühgeborenen mit Hirnblutungen (1) wurde in unserer Einrichtung diese Intervention im Rahmen individueller Heilversuche im Jahr 2017 eingeführt. Bei Patienten mit intraventrikulärer Hirnblutung (IVH) werden nach $\mathrm{Zu}$ stimmung der Kindeseltern alle 3-4 h jeweils 0,1 ml frische Muttermilch in jedes Nasenloch appliziert.

Zielstellung: Ziel der vorliegenden Arbeit ist ein Vergleich der Patientenpopulationen vor und nach Einführung dieser Intervention. Die Ergebnisse sollen die Grundlage darstellen für eine prospektive klinische Studie. Methoden: Es wird eine retrospektive Analyse durchgeführt. Hierbei werden Patienten betrachtet, die zwischen März 2016 und Februar 2019 in der Schädelsonographie eine $\mathrm{IVH}_{\mathrm{II}}^{\circ}-\mathrm{III}^{\circ}$ aufwiesen. Dabei werden Patienten gegenübergestellt, welche intranasal Muttermilch erhielten (MM+) oder nicht (MM-). Patienten der MM+ Gruppe gelten als Fallgruppe, während Patienten ohne intranasale Muttermilchgabe (MM-) als Kontrollgruppe betrachtet werden. Die statistischen Analysen werden mit dem R Softwarepaket durchgeführt. Das Signifikanzlevel wird für alle Berechnungen auf $a<0,05$ festgelegt.

Ergebnisse: Innerhalb der analysierten drei Jahre wurden insgesamt 406 Frühgeborene $<1500 \mathrm{~g}$ betreut. Davon erfüllten 36 Patienten $(8,9 \%)$ die Einschlusskriterien; 19 erhielten intranasal Muttermilch, 17 nicht. Die perinatalen Parameter unterscheiden sich nicht signifikant zwischen beiden Gruppen. Die intranasale MM-Applikation wurde gut toleriert.

Der Vergleich beider Gruppen zeigt tendenziell weniger Shuntversorgungen $(\mathrm{MM}+5 \%$ vs. MM- $18 \%, p=0,33)$ in der MM-behandelten Gruppe, der Unterschied ist bei der kleinen Fallzahl jedoch nicht statistisch signifikant. 
Diskussion: Die intranasale Applikation von Muttermilch scheint sicher durchführbar. Die vorliegende Analyse bestätigt die Ergebnisse vorangehender Untersuchungen und unterstützt die Notwendigkeit einer randomisierten kontrollierten Studie zu Überprüfung der Wirksamkeit dieser Intervention.

\section{Literatur \\ 1. Keller T, Körber F, Oberthuer A et al (2019) Eur J Pediatr 178:199. https:// doi.org/10.1007/s00431-018-3279-7}

\section{DGKJ-PO 55 \\ Neonatale Staphylococcus-aureus-Sepsis nach mütterlicher Mycophenolat-Mofetil Einnahme in der Schwangerschaft}

Franziska Roche', Silvia Poralla ${ }^{2}$, Till Dresbach ${ }^{2}$, Andreas Müller ${ }^{2}$

'Universitätsklinikum Bonn, Neonatologie und pädiatrische Intensivmedizin, Bonn, Deutschland; ${ }^{2}$ Universitätsklinik Bonn, Neonatologie und pädiatrische Intensivmedizin, Pädiatrische Infektiologie, Bonn, Speyer

Einleitung: Die neonatale Sepsis ist aufgrund ihrer hohen Mortalität eine gefürchtete Komplikation des Frühgeborenen. Iatrogene Immunsuppression durch Mycophenolat-Mofetil (MMF) erhöht das Risiko für eine Sepsis durch eine verminderte B- und T-Zell-Aktivität. MMF hemmt selektiv, nicht-kompetitiv und reversibel die Inosinmonophosphat-Dehydrogenase, ein Schlüsselenzym der Purin Synthese und somit der Proliferation von B- und T-Lymphozyten. MMF wird off-lable bei Erwachsenen mit Systemischem Lupus Erythematodes (SLE) unter anderem bei Lupus Nephritis eingesetzt. Die Therapie eines SLE mit MMF in der Schwangerschaft wird, aufgrund einer möglichen Embryopathie, sowie assoziierten Frühgeburten mit erniedrigtem Geburtsgewicht, nicht empfohlen. Bei einer mütterlichen Therapie bis zur Entbindung können beim Neugeborenen MMF Plasmaspiegel erreicht werden, die im therapeutischen Bereich liegen. Wir berichten über den Verlauf eines $31+4$ SSW Frühgeborenen, dessen Mutter während der Schwangerschaft aufgrund einer schweren Lupus Nephritis mit MMF behandelt wurde.

Fallbeschreibung: Der Patient wurde mit $31+4$ SSW bei intrauteriner Wachstumsretardierung und pathologischen Dopplerflüssen in der Sonographie per Sectio geboren. Im ersten Trimenon wurde bei der Mutter die Erstdiagnose eines SLE mit schwerer Lupusnephritis Typ IV und V.a. Anti-Phospholipid-Syndrom gestellt. Hierauf erfolgte eine Medikation mit Prednisolon, Hydroxychloroquin, Mycophenolat-Mofetil und Clexane. Der MMF Spiegel bei unserem Patient lag am 1. Lebenstag oberhalb des therapeutischen Bereichs bei 4,0 mg/l (Ref.: 1,3-3,5 mg/l). Nach zunächst dem Gestationsalter entsprechend adäquatem Verlauf, entwickelte unser Patient am 4. Lebenstag eine Late-Onset-Sepsis, welche sich klinisch durch Sättigungsabfälle, Herzfrequenzschwankungen, sowie eine gerötete Eintrittsstelle des Venenkatheters zeigte. Laborchemisch war das C-reaktive Protein auf 12,9 mg/l erhöht, es bestand eine relative Lymphopenie bei Leukopenie und in der Blutkultur konnte Staphylococcus aureus nachgewiesen werden. Unter antibiotischer Therapie mit Piperacillin/Tazobactam erholte sich der Patient rasch und komplikationslos. Unser Patient zeigte in der Entwicklungsneurologischen Untersuchung nach 6 Monaten ein altersentsprechendes Verhalten.

Fazit: Es ist möglich, dass die stattgehabte Sepsis unseres Patienten mit der mütterlichen MMF Einnahme assoziiert war. MMF sollte als plazentagängiges und potentiell teratogenes Medikament in der Schwangerschaft grundsätzlich nicht angewendet werden. Wenn eine MMF Einnahme unumgänglich ist, sollte in der Neonatalperiode ein besonderes Augenmerk auf das erhöhte Infektionsrisiko gelegt werden.

\section{DGKJ-PO 56}

Late onset - big impact: Septische Arthritis und Ventrikulitis durch Gruppe B Streptokokken bei einem fünf Wochen alten Säugling

Julian Sommer', Boris Wittekindt', Christian Wölke', Stephan Göttig'², Thomas Wichelhaus ${ }^{2,3}$, RolfSchlößer $^{4}$

'Uniklinik Frankfurt, Neonatologie, Frankfurt, Deutschland; ${ }^{2}$ Uniklinik Frankfurt, Institut für medizinische Mikrobiologie und Krankenhaushygiene, Frankfurt; ${ }^{3}$ niklinik Frankfurt, Institut für medizinische Mikrobiologie und Krankenhaushygiene, Frankfurt, Deutschland; ${ }^{4}$ Uniklinik Frankfurt, Neonatologie, Frankfurt, Deutschland

Hintergrund: Bei mit Streptokokken der Lancefield-Gruppe B (GBS) besiedelten Schwangeren kann es durch vertikale Übertragung unter der Geburt beim Neugeborenen zu Infektionen mit unterschiedlichen klinischen Verläufen kommen, wobei sich früh manifestierenden Erkrankungen (early-onset) primär als Pneumonie oder Sepsis ablaufen, während spätere Manifestationen (late-onset) häufiger mit einer Meningitis einhergehen. Wir berichten von einem fünf Wochen alten Säugling mit komplizierten Verlauf einer GBS-Infektion mit septischer Arthritis und Ventrikulitis.

Fallbericht: Ein reif geborenes Kind wurde nach unkompliziertem Spontanpartus am vierten Lebenstag gesund aus der Geburtsklinik entlassen. In der fünften Lebenswoche erfolgte die Einweisung bei Gewichtsverlust und einer Schwellung am rechten Oberarm.

Untersuchungsbefund bei Aufnahme: Dystropher Säugling in stabilem Allgemeinzustand, afebril, eupnoeisch, peripher gut durchblutet. Einschränkung der Bewegung des rechten Arms, hier tastete sich über dem Epicondylus medialis eine Schwellung. Bis auf eine diskret gespannte Fontanelle sonst keine pathologischen Befunde in der klinischen Untersuchung.

Labor: CRP 6,5 mg/dl, Leukozyten 12,8/nl, Natrium 128 mmol/l; Röngten: keine Fraktur; Sonographie: Erguss im rechten Ellenbogengelenk mit Periostabhebung und deutliche Erweiterung der inneren Liquorräume. Nach Beginn der antibiotischen Behandlung (s. u.) erfolgte eine MRT-Untersuchung des Schädels, in der ein Hydrocephalus occlusus diagnostiziert wurde, zu dessen Behandlung drei Rickham-Reservoire implantiert wurden. Im Liquor waren Leukozytenzahl $(1427 / \mu \mathrm{L})$ und Gesamtprotein $(20 \mathrm{~g} / \mathrm{l})$ stark erhöht. Die mikrobiologische Diagnostik erbrachte einen Nachweis von GBS-DNA per PCR im Liquor. Zusätzlich konnte GBS aus dem Rachenabstrich kulturell angezüchtet werden.

Wir behandelten mit Ampicilin, Cefotaxim, Clindamycin und Fosfomycin über 4 Wochen. Hierunter normalisierten sich die Liquorbefunde langsam. Bei weiterhin behindertem Liquorabfluss erfolgte nach weiteren 4 Wochen die operative Anlage von zwei VP-Shunts.

Bei Entlassung hatte das Kind eine gute Gewichtsprogression und bis auf einen deutlichen Strabismus keine neurologischen Auffälligkeiten. Die Arthritis war klinisch und sonographisch ausgeheilt.

Diskussion: In Deutschland wird nur bei einem Teil der Schwangeren ein GBS Screening durchgeführt. Durch die Antibiotika-Therapie während der Geburt kann die Inzidenz der early-onset Sepsis reduziert werden, die Häufigkeit der selteneren late-onset Sepsis wird hierdurch allerdings kaum beeinflusst. Aktuell sind daher Impfstoffe in der Entwicklung, die einen Schutz von Schwangeren und Neugeborenen gegen Infektionen mit GBS bewirken könnte.

Der molekularbiologische Nachweis von bakterieller DNA kann auch nach Beginn einer Antibiotika-Therapie Erreger identifizieren.

\section{DGKJ-PO 57}

Messung volatiler organischer Substanzen mittels Multikapillarsäulen-gekoppelter lonenmobilitätsspektrometrie: Potential zur nicht-invasiven Diagnostik in der Neonatologie

Michelle Bous

Universitätsklinikum d. Saarlandes, Klinik für Allgemeine Pädiatrie und Neonatologie, Homburg, Deutschland 
Messung volatiler organischer Substanzen mittels Multikapillarsäulen-gekoppelter Ionenmobilitätsspektrometrie: Potential zur nicht-invasiven Diagnostik in der Neonatologie

Fragestellung: Blutentnahmen bei Frühgeborenen können Stress und Anämien verursachen und sich somit negativ auf die Kurz- und Langzeitentwicklung auswirken. Daher ist eine nicht-invasive Diagnostik erstrebenswert. Großes Potential bergen flüchtige volatile Substanzen (volatile organic compounds, VOCs), die im Körper bei physiologischen und pathophysiologischen Prozessen entstehen und über Haut, Urin, Stuhl und Ausatemluft an die Umwelt abgegeben werden. Ziel ist es, eine Methode zur VOC-Analyse zu entwickeln, mit welcher Infektionen und andere Krankheitszustände detektiert werden können.

Material und Methode: Mittels Multikapillarsäulen-gekoppelter Ionenmobilitätsspektrometrie (MCC-IMS) können VOCs bereits in extrem geringen Konzentrationen (pg/l) detektiert werden. Wir haben VOCs in Urinund Stuhlproben Frühgeborener mittels MCC-IMS analysiert und die statistische Auswertung der Daten unter Verwendung des Mann-Whitney-U-Tests und Bonferroni-Korrektur durchgeführt. Einschlusskriterien waren neben der Betreuung auf den neonatologischen Stationen des Universitätsklinikums des Saarlandes ein Geburtsgewicht $<2000$ g oder ein Gestationsalter $<32$ Wochen.

Ergebnisse: Es wurden VOC-Profile von 133 Stuhl- und Urinproben von insgesamt 12 Frühgeborenen untersucht. Zunächst erfolgte die stufenweise Etablierung der MCC-IMS-Methode zur Analyse der VOCs in Windelproben der Frühgeborenen. Im Sinne eines „proof of principle“-Experiments zeigten sich signifikante Ergebnisse im Signifikanzlevel von $p<0,001$. Es konnte demonstriert werden, dass der Inhalt der Windel (Urin/Stuhl) anhand des VOC-Musters klassifiziert werden kann, eine Unterscheidung zwischen leerer und voller Windel konnte ebenfalls vorgenommen werden.

Diskussion: Wir haben eine Umgebungsluft-unabhängige Methode zur Messung von VOCs an Bioproben von Frühgeborenen mittels Multikapillarsäulen-gekoppelter Ionenmobilitätsspektrometrie (MCC-IMS) entwickelt. Die Ergebnisse stellen einen innovativen Ansatz zur nicht-invasiven Diagnostik in der Neonatologie dar. Durch die Erkenntnis, dass man den Inhalt der Windeln mittels MCC-IMS nachweisen kann, könnte zukünftig ein bedarfsgerechtes Windelwechseln erfolgen und somit unnötiges Öffnen des Inkubators, was mit Stress für das Kind sowie einem hygienischen Risiko einhergeht, vermieden werden. Von großer klinischer Bedeutung könnte die Anwendung der VOC-Analyse in der Diagnostik verschiedener Krankheitsentitäten, wie z. B. Sepsis sein. Die Methode birgt daher großes Potential und könnte bei der Diagnostik weiterer Krankheitsbilder erprobt werden.

Schlussfolgerungen: Profile volatiler organischer Substanzen können zuverlässig und Umgebungsluft-unabhängig an Bioproben von Frühgeborenen erhoben werden und könnten sich zur Suche nach Biomarkern in der Neonatologie eignen.

\section{DGKJ-PO 58}

Cutis marmorata teleangiectatica congenita (CMTC) - Fallbericht eines betroffenen Kindes mit angeborenen vaskulären Malformationen aus dem Formenkreis dermatomaler oder segmentaler Teleangiektasien, Epilepsie und Hemihypertrophie der Extremitäten

\section{Richard Eyermann}

'Dr. Eyermann München, Kinder- und Jugendmedizin, Kinderkardiologie, Sportmedizin, München, Deutschland; ${ }^{2}$ Klinik Schönsicht Berchtesgaden, Rehabilitation für Kinder und Jugendliche, AHB, Kind-Mutter/VaterRehabilitation, München, Deutschland

Erstbeschreiber: Van Lohuizen, 1922

Definition: Konnatale, durch Teleangiektasien und Phlebektasien hervorgerufene, generalisierte oder lokalisierte, nicht selten systematisierte (entweder entlang der Dermatomgrenzen ausgebildet oder den BlaschkoLinien folgend), netzförmige Zeichnung der Haut. Die Dystrophie (Unterscheidung zur passageren Livedo reticularis des Neugeborenen) kann isoliert an der Haut auftreten oder mit mesodermalen oder neuroektodermalen Anomalien einhergehen. Nicht selten können Atrophien in der befallenen Haut nachgewiesen werden. Auch lokalisierte Atrophien des subkutanen Fettgewebes, der angrenzenden Muskulatur sowie des knöchernenen Skeletts sind beschrieben (s. a. Adams-Oliver-Syndrom).

Manifestation: Ab Geburt, keine Geschlechtsbevorzugung.

Lokalisation: Sowohl lokalisierte, unilaterale Formen (meist segmental oder den Blaschko-Linien folgend), v. a. an den Extremitäten; auch isoliert am Stamm. Selten ist generalisiertes Auftreten möglich.

Klinisches Bild: Integument: Unsymmetrisch, auch segmental verteilte oder systematisierte marmorierte Haut mit Teleangiektasien und Phlebektasien; häufig auffallend dünne, durchscheinende (atrophische) Haut mit deutlicher Venenzeichnung.

Seltener sind eingestreute Spinnennävi oder prominente Venen.

Bei Jugendlichen und Erwachsenen kann eine deutliche Fettgewebs- und Muskelatrophie vorhanden sein.

Nicht ganz selten ist die Cutis marmorata teleangiectatica congenita mit einem melanozytären Naevus oder einem Naevus anämicus kombiniert (s. a. pigmentär vaskuläre Phakomatose)

Extrakutane Manifestationen: Genitoanale Anomalien, Skelettveränderungen, Lipome, Hyperkalzämie, Glaukom, Missbildungen wie Hemiatrophie, Hemihypertrophie betroffener Extremitäten, neuromuskuläre Störungen. Vereinzelt Ausprägung eines progerieartigen Aspektes.

Histologie: Vermehrte, zum Teil lakunär erweiterte Kapillaren, Venulen in der Dermis und Subkutis.

Diagnose: Bei Säuglingen ist die Unterscheidung zur reaktiven Livedo reticularis wichtig.

Differentialdiagnose: Livedo reticularis des Neugeborenen (reaktiv, rückbildungsfähig in den ersten Lebensmonaten)

Genuine diffuse Phlebektasie

Acrodermatitis chronica atrophicans (nicht angeboren, DD bei Erwachsenen, die sich erstmals mit den Erscheinungen vorstellen; fehlende Serologie, Histologie ist für die chronische Borreliose diagnostisch)

Livedo racemosa (nicht angeboren, Histologie ist diagnostisch, weitere Zeichen der Systemvaskulitis); s. u. Livedosyndrome.

Therapie: Symptomatisch.

Verlauf/Prognose: Komplette Rückbildung in 50 \% der Fälle in den ersten Lebensjahren. Ein Teil der Fälle persistiert oder entwickelt sich progredient (Atrophie von Haut, Fettgewebe, Muskulatur und/oder Skelettsystem). Inwieweit diese klinisch differenten Verläufe sich genetisch unterscheiden ist bisher nicht geklärt.

Hinweis(e): Nach Kienast und Höger werden Major- und Minor-Kriterien aufgeführt.

Major-Kriterien: Kongenitales retikuläres Erythem, keine Venektasien, keine Reaktion auf Erwärmung.

Minor-Kriterien: Abheilung über 2 Jahre, Teleangiektasien, Naevus flammeus andernorts, Ulzeration, Atrophie der Haut (Kienast u. Höger 2018).

\section{DGKJ-PO 59}

\section{Angeborenen multiplen Exostosen (MO) - Häufigkeit und Art} stationärer Behandlungen bei Kindern und Jungendlichen

\section{Steffen Wahler', Lothar Seefried ${ }^{2}$}

${ }^{1}$ St. Bernward GmbH, Epidemiologie, Hamburg, Deutschland;'²Universität Würzburg, Orthopädie, Würzburg, Deutschland

Einführung: Angeborene multiple Exostosen bzw. Multiple Osteochondrome (MO) bezeichnet eine autosomal-dominant vererbte Erkrankung, charakterisiert durch multifokales Auftreten primär benigner Osteochondrome. Die typische Lokalisation in der Nähe der Wachstumsfugen kann einhergehen mit gestörtem Längenwachstum, Bewegungseinschränkungen und Schmerzen. Ursächlich sind loss-of-function Mutationen in den Genen EXT1 und EXT2 die für Glykosyltransferasen kodieren. Die Inzidenz wird mit 1:50.000 angenommen. Bei $70 \%$ der Patienten liegt eine familiäre Form vor, bei $30 \%$ eine Neumutation. Die individuelle Ausprägung der MO ist sehr variabel. Die wesentliche Therapieform ist die operative Entfernung der Osteochondrome. 
Es gibt für Deutschland kaum epidemiologische Daten zur MO gibt. Hier sollen die stationären Aufenthalte von Patienten mit dem spezifischen ICD-Code (Q78.6) über 12 Jahre untersucht werden.

Methoden: Daten der stationären Versorgung des statistischen Bundesamtes 2005-2016 und die Qualitätsberichte für das Jahr 2016 wurden auf MO-Kodierungen ausgewertet. Analyse mit Microsoft-Excel und Access (V 2016)

Ergebnisse: Im Jahr 2016 wurden 269 stationäre Fälle (2005: 200) mit MO als Hauptdiagnose (HD) und 230 als Nebendiagnose (ND) kodiert $(2005=238)$. Die Gesamtzahl der Fälle schwankte im Beobachtungszeitraum zwischen 393 und 518 pro Jahr, zuletzt steigend.

Die Patienten wurden in 87 verschiedenen stationären Einrichtungen behandelt (HD) wobei $95 \%$ der Behandlungen in chirurgischen oder orthopädischen Fachabteilungen, bzw. Kinderfachabteilungen erfolgten. Kodiert wurden zu 97 \% operative DRG des Muskel- und Skeletsystems. $24 \%$ der Fälle wurden in Universitätseinrichtungen versorgt.

Das Durchschnittsalter lag 2016 bei 18,5 Jahren, Median bei 20 Jahren (nur HD). Männliche Patienten waren im Mittel 17,7 Jahre alt, weibliche 20,1 Jahre. Es gibt eine Lücke in der Altersverteilung mit vergleichsweise wenigen Versorgungen in der Transitionsphase vom 20. bis 25. Lebensjahr. Seit 2005 hat sich das Altersmittel tendenziell um knapp zwei Jahre verjüngt. In 2016 betrafen 64,3 \% der Fälle (HD) Männer. Die Einbeziehung weiterer Jahre bestätigt, dass stets etwa $60 \%$ der Behandlungen männliche Patienten betreffen und diese zum Behandlungszeitpunkt im Mittel zwei Jahre jünger sind als die Frauen. Die durchschnittliche Verweildauer war bei beiden Geschlechtern mit 4,1 Tagen gleich.

Schlussfolgerungen: Stationär operationsbedürftige Fälle von MO sind in Deutschland selten aber konstant mit leicht steigender Tendenz. Bemerkenswert sind die Ungleichverteilung der Geschlechter vor der Hintergrund einer autosomalen Erkrankung sowie das zunehmend geringere Alter der Männer bei stationären Eingriffen. Die Lücke im Transitionsalter könnte man u. a. auf das Fehlen eines krankheitsspezifischen Transitionsprogrammes zurückführen. Zur Ermittlung der exakten Prävalenz wären gezielte Forschungsansätze oder ein systematisches Register erforderlich.

\section{DGKJ-P0 60}

Seltene Differenzialdiagnose der schweren Infektion beim Neugeborenen - ein Fallbericht

Sebastian Friedrich ${ }^{1}$, Niklas Gäbler², Ulrike Teufel-Schäfer', Christoph Müller', Anton Härtling ${ }^{3}$

${ }^{1}$ Klinik für Allgemeine Kinder- und Jugendmedizin, Zentrum für Kinderund Jugendmedizin, Universitätsklinikum Freiburg, Medizinische Fakultät, Albert-Ludwigs-Universität Freiburg, Deutschland, Klinik für Allgemeine Kinder- und Jugendmedizin, Zentrum für Kinder- und Jugendmedizin, Freiburg, Deutschland:'2Klinik für Kinder- und Jugendmedizin, St. Josefskrankenhaus, RKK Klinikum, Freiburg, Deutschland, Klinik für Kinder- und Jugendmedizin, Freiburg, Deutschland; ${ }^{3}$ Klinik für Kinder und Jugendmedizin, St. Josefskrankenhaus, RKK Klinikum, Freiburg, Deutschland, Klinik für Kinder und Jugendmedizin, Freiburg, Deutschland

Hintergrund: Das Food Protein Induced Enterocolitis Syndrome (FPIES) gehört zu den nicht IgE-vermittelten Nahrungsmittelallergien. Genaue epidemiologische Zahlen fehlen, aber mit Inzidenzen von max. 15/100.000 gehört das FPIES zu den eher seltenen Erkrankungen. Das erste Auftreten liegt meist in den ersten 9 Lebensmonaten. Als auslösende Substanzen sind u. a. Kuhmilchprotein (KMP) und Soja beschrieben. Das klinische Bild des akuten FPIES mit massivem Erbrechen, Durchfällen, Apathie, Hypovolämie und Hypothermie macht die Unterscheidung zu infektiösen Ursachen im klinischen Alltag zu einer Herausforderung. Wir berichten von einem Neugeborenen mit erstem Auftreten eines akuten FPIES, begleitet von massiven Volumenverlusten und Hypernatriämie.

Fall: Die Vorstellung eines 25 Tage alten männlichen Reifgeborenen erfolgte mit seit dem Aufnahmetag bestehendem Erbrechen und wässrigen Diarrhoen, zudem erschwerter Atmung und marmoriertem Hautkolorit Initial zeigten sich stabile Vitalparameter, eine mittelgradige Exsikkose, der CRP-Wert mit 16,5 mg/l leicht erhöht. Unter Glucose-Elektrolytlö- sung weiter massive Durchfälle, zeitweise Ausscheidung von $1300 \mathrm{ml} / 24 \mathrm{~h}$, entsprechend $42 \%$ des Körpergewichts. Bei CRP-Anstieg $(91 \mathrm{mg} / \mathrm{l}) \mathrm{er}-$ folgte zusätzliche Sepsis-Diagnostik (Lumbalpunktion, Blutkultur, Urin, Stuhl). In der Folge kam es zur Entwicklung einer schweren Hypernatriämie $(167 \mathrm{mmol} / \mathrm{l})$ mit Hyperchlorämie, Laktatazidose und Methämoglobinämie. Es wurde eine empirische antibiotische Therapie begonnen, zudem Umstellung der Flaschennahrung auf kuhmilchproteinfreie Formula. Darunter kam es zu einem deutlichen Rückgang der Symptome und Abfall des CRP. Entlassung in gutem Allgemeinzustand. Kein Keimnachweis in o.g. Materialien. 4 Wochen später Wiederaufnahme zur Provokation mit kuhmilcheiweißhaltiger Nahrung. Nach Gabe von $1 \times 15 \mathrm{ml}$ und $2 \times 30 \mathrm{ml}$ (Formula, 1,2 g/100 ml) erneut Entwicklung von Erbrechen (Latenz $1 \mathrm{~h}$ ), profusen Durchfällen (Latenz $4 \mathrm{~h}$ ) und deutlicher Marmorierung. Anstieg von Serumnatrium $(151 \mathrm{mmol} / \mathrm{l})$, Chlorid, Laktat und Methämoglobin. Innerhalb von $36 \mathrm{~h}$ vollständiger Rückgang der Symptome. Die klinische Präsentation erfüllt damit alle Kriterien für die Diagnose eines akuten FPIES durch Kuhmilchprotein.

Schlussfolgerung: Die Differenzialdiagnose der Neugeboreneninfektion sollte neben infektiösen auch allergische Ursachen beinhalten. Im vorliegenden Fall waren unter anderem die gastroenteritischen Symptome ohne Erregernachweis, als auch die Methämoglobinämie hinweisgebend. Neben empirischer antibiotischer Therapie muss daher auch eine kuhmilchproteinfreie Nahrung in Betracht gezogen werden. Die Diagnosestellung kann dann durch Provokation unter stationären Bedingungen erfolgen. Eine allergologische Beratung der Eltern ist geboten, um über das Krankheitsbild aufzuklären und im Notfall auch Kollegen auf diese seltene Ursache für eine Sepsis-ähnliche Präsentation hinweisen zu können. Die Prognose des FPIES ist gut.

\section{DGKJ-PO 61}

\section{Individueller Heilversuch: Erfahrungen mit Therapie des} kongenitalen Plasminogenmangels (PM) bei einem 11 Monate alten Mädchen mit Hydrocepahlus (HC) und Konjunktivitis lignosa (KL)

Madlen Reschke', Volker Schuster ${ }^{2}$, Hans Christoph Ludwig ${ }^{3}$, Westekemper Henrike $^{4}$

'Universitätsklinikum Essen, Klinik für Kinder und Jugendmedizin, HämatoOnkologie, Essen, Deutschland:'2Universitätsklinik und Poliklinik für Kinder und Jugendliche Leipzig, Neuropädiatrie, Leipzig, Deutschland; ${ }^{3}$ Universit ätsmedizin Göttingen, Georg-August-Universität Göttingen, Pädiatrische Neurochirurgie, Göttingen, Deutschland; ${ }^{4}$ Universitätsklinikum Essen, Zentrum für Augenheilkunde, Essen, Deutschland

Einleitung: Bei homozygoter oder compound-heterozygoter Mutation des PLG Gens kommt es zu deutlichem PM. Physiologisch entstandenes Fibrin(ogen) wird unzureichend abgebaut. Es entstehen, v. a. im Rahmen von Wundheilung, fibrinreiche, pseudomembranöse Läsionen auf sämtlichen Schleimhäuten (z. B. Augen, Trachea, Gingiva). Deshalb sollte auf jegliche chirurgische Interventionen, soweit wie möglich, verzichtet werden. Häufig fallen Kinder initial mit einem Hydrocephalus occlusus auf. Nach ventrikuloperitonealer Shuntanlage erschweren Membranbildungen die peritoneale Liquorresorption (fibrin(ogen)reicher Aszites).

Konsekutiv entwickelt sich eine KL bds., die unbehandelt zu Erblindung führen kann. Bisher gibt es keine effektive, zugelassene Behandlung. Klinische Präsentation: 1. Kind nicht konsanguiner Eltern. Geburt per elektiver Sektio in der 35. SSW wegen Hydrocephalus occlusus. VP Shunt Anlage 10 Tage postnatal. KL bds. seit dem 4. Lebensmonat, einhergehend mit stetiger Unruhe und Schmerzen. Wiederholte Abtragung der okularen Membranen, die trotz intensiver Lokaltherapie (Heparin-, Cortisontropfen etc.) schnell rezidivierten. Komplikationen im Verlauf: Erhöhter Augeninnendruck durch lokale Cortisontherapie. 2. Gedeihstörung bei massivem Aszites wegen peritonealer Resorptionsstörung. Im 11. Lebensmonat Nahrungsverweigerung (außer Muttermilch in kleinen Mengen) Körpergewicht $<5$. P (inklusive massivem Aszites); Körperlänge $<1$. P; Kopfumfang 84. P.. Die Plasminogenrestaktivität im Plasma betrug zwi- 
schen $13-30 \%$. Humangenetisch fand sich eine heterozygote compound Mutation im PLG Gen.

Seit dem 11. Lebensmonat erfolgt iv Substitution mit lyophilisiertem, humanen Glu Plasminogen der Firma Prometic ${ }^{\circ}$ (noch keine FDA Zulassung). Diese führte zu deutlicher Besserung des Allgemeinzustandes, wie zuvor publiziert1. Nach $15 \mathrm{~d}$ hatte sich der Augenbefund normalisiert. Der Aszites war deutlich rückläufig. Die selbständige Nahrungsaufnahme normalisierte sich nach wenigen Wochen.

Die initial hohe Substitutionsfrequenz alle $48 \mathrm{~h}$ konnte nach Abklingen der Krankheitsmanifestationen auf aktuell alle 5 Tage reduziert werden. Die Plasminogenrestaktivität im Plasma betrug $24 \mathrm{~h}$ nach der Gabe $(6,6 \mathrm{mg} /$ khKG) etwa $100 \%$, nach 48 h etwa $45 \%$, nach 5 d etwa $20 \%$. Therapieauslassversuche führten nach ca. 5 Tagen zu erneuter KL und zunehmendem Aszites. Die Mutter verabreicht das Medikament eigenständig über einen Portkatheter. Zusammenfassung: Kongenitaler Plasminogenmangel ist eine leidvolle und potentiell lebensbedrohliche Erkrankung, die bei Patienten mit Hydrocephalus okklusus und therapieresistenter Konjunktivitis lignosa ausgeschlossen werden sollte. Die aktuell zugelassenen Therapieoptionen können keine akzeptable Lebensqualität ermöglichen. Die Behandlung mit lyophilisiertem humanen Plasminogen ist relativ einfach, sicher und sehr effektiv.

Plasminogen replacement therapy for the treatment of children and adults with congenital plasminogen deficiency. Amy D. Shapiro, 1 Charles Nakar,1 Joseph M. Parker,2 Gary R. Albert,2 John E. Moran,3 Karen Thibaudeau,3 Neelam Thukral,1 Brandon M. Hardesty,1

\section{DGKJ-PO 62}

\section{Wenn Schönheit schadet - indirekte Quecksilbervergiftung durch Kosmetika}

Elisabeth Asenbauer³, Stefan Rakete', Stephan Böse-O'Reilly', Jochen Peters², Steffen Leiz ${ }^{2}$

'Institut und Poliklinik für Arbeits-, Sozial- und Umweltmedizin, Klinikum der Universität München, Analytik und Monitoring, München, Deutschland;' ${ }^{2}$ linikum Dritter Orden München Nymphenburg, Klinik für Kinder- und Jugendmedizin, München, Deutschland ${ }^{3}$ München, Deutschland; ${ }^{4}$ Institut und Poliklinik für Arbeits-, Sozial- und Umweltmedizin, Klinikum der Universität München, Globale Umweltmedizin, München, Deutschland

Wir berichten über einen 4 Jahre alten Jungen, der seit Juli 2018 folgende Symptome entwickelte: rasche Ermüdbarkeit, Appetitminderung und $2 \mathrm{~kg}$ Gewichtsverlust innerhalb von vier Wochen, vermehrtes Schwitzen, Bauchschmerzen, Beinschmerzen sowie ausgeprägte Verhaltensauffälligkeiten (Irritabilität und Missmutigkeit). Zudem bestanden eine arterielle Hypertonie und eine Tachykardie. Es erfolgte eine ausführliche Labordiagnostik unter besonderer Berücksichtigung entzündlicher (infektiöser und autoimmuner) und hämato-onkologischer Ursachen (einschließlich KMP). Die einzigen auffälligen Befunde waren erhöhte Catecholaminkonzentrationen im Urin, passend zur arteriellen Hypertonie und Tachykardie. Die bildgebende Diagnostik - inklusive Ganzkörper-MRT - ergab keinen wegweisenden Befund. Schließlich konnten erhöhte QuecksilberKonzentrationen im Blut und Urin nachgewiesen werden, die deutlich über den HBM-II Werten lagen. Auch die klinische Symptomatik war mit einer chronischen Quecksilberintoxikation zu vereinbaren. Bei der Mutter und dem Bruder fanden sich ebenfalls erhöhte Werte für Quecksilber. Durch eine Therapie mit Dimaval ${ }^{\circledR}$ (DMPS) normalisierten sich die Quecksilber-Konzentrationen innerhalb von 2 Monaten und die klinische Symptomatik bildete sich vollständig zurück.

Für potentielle Quecksilberquellen wie Thermometer, Batterien oder Leuchtmittel ergaben sich keine Hinweise. Es konnte eine hautaufhellende Gesichtscreme der Mutter aus dem Kosovo mit einem Quecksilbergehalt von ca. $18 \%$ identifiziert werden. Allerdings verneinte die Mutter die Applikation der Creme am Kind, so dass nach weiteren Quellen in der häuslichen Umgebung gesucht wurde. Dabei zeigte sich eine fast hundertfach erhöhte Quecksilber-Konzentrationen im Hausstaub. In einer Messung der Raumluft in der Wohnung der Familie fanden sich jedoch keine Wer- te oberhalb der Hintergrundbelastung, so dass eine inhalative Exposition ausgeschlossen werden konnte. Nachdem die Creme eliminiert wurde, zeigten sich rückläufige Quecksilber-Konzentrationen in Blut, Urin und Hausstaub, sodass die Creme als einzige Quelle bestätigt werden konnte. Zusammenfassend gehen wir von einer indirekten, akzidentellen Quecksilbervergiftung durch Hautkontakt zwischen Mutter und Kind aus, was nach unserer Kenntnis in der Literatur noch nicht beschrieben ist. Die erhöhten Quecksilber-Konzentrationen im Hausstaub sind am ehesten durch abgeschilferte Hautzellen zu erklären.

\section{DGKJ-PO 63}

\section{Psyche versus Physis Das „clenched-fist-syndrome“}

\section{Felix Schriegel', Anja Lange', Heimke Osten ${ }^{2}$, Norbert Utzig', Knud} Linnemann', Matthias Heckmann'

'Universitätsklinikum Greifswald, Kinder- und Jugendmedizin, Greifswald, Deutschland;'niklinikum Greifswald, Kinder- und Jugendmedizin, Greifswald, Deutschland

Hintergrund: Die Unterscheidung einer „somatischen“ Erkrankung von psychischen Störungen kann eine Herausforderung sein und ist nicht immer möglich. Ein Paradebeispiel hierfür ist das "clenched fist syndrome“/ „psycho-flexed hand syndrome“. Es ist gekennzeichnet durch Schmerzen, Schwellung und paradoxer Steifheit nach einem „Bagatelltrauma“ und manifestiert sich in Form einer stark geballten Faust und flektierten Fingern, die aktiv und passiv nicht gelöst werden können. Bei diesem in der Pädiatrie seltenen Syndrom besteht zunächst kein Leidensdruck und es findet sich kein pathomorphologisches Korrelat. Bei Persistenz der Symptomatik sind als Komplikationen Funktionseinschränkungen, Ulzerationen und Infektionen der betroffenen Hand beschrieben, welche sogar schon zu Amputationen führten.

Fallvorstellung:

Anamnese: Ein 15-jähriges bisher gesundes Mädchen klagte im Anschluss an einen Stromunfall über ein Taubheitsgefühl und Bewegungseinschränkungen der betroffenen Hand, die sich seit dem in einer Pfötchenstellung befand.

Befund: 15-jähriges Mädchen in gutem Allgemeinzustand und adipösen Ernährungszustand (KG $95 \mathrm{~kg}$, KL $174 \mathrm{~cm}$, BMI 31,4) Temperatur $36,7^{\circ} \mathrm{C}$, RR 116/63 mmHg, HF $71 \mathrm{bpm}, \mathrm{SaO} 297 \%$. Parästhesie in der rechten Hand bis zum Handgelenk. Flexion der Finger der rechten Hand, weder passives noch aktives Lösen möglich. Hoher dynamischer Tonus in der rechten Hand, der bei Kraftprüfung der linken Hand nachließ. Keine Strommarken.

Diagnostik: Labor: Normalbefunde für: Blutbild, BZ, Troponin I, CK, LDH, Drogenscreening. EKG: Normalbefund, Nervenleitgeschwindigkeitstestung: $\mathrm{N}$. ulnaris $57 \mathrm{~m} / \mathrm{s}$ beidseits, motorische Latenzen $\mathrm{N}$. ulnaris und N. medianus beidseits 2,1-2,5 ms: Normalbefund; EEG: okzipital betonte alpha-beta-Grundaktivität um 9-10/sek., Amplitude 20-40 $\mu \mathrm{V}$, Temporo-okzipital dysrhythmisches, und spannungslabiles EEG mit deutlicher Beta-Auflagerung.

Verlauf: Im Schlaf zeigte sich eine gelöste Handhaltung, im Wachzustand konnten die Finger weder aktiv noch passiv bewegt werden. Die Patientin zeigte einen auffallend geringen Leidensdruck. Im weiteren Verlauf spontane Rückbildung.

Diskussion: Differentialdiagnostisch schlossen wir eine rheumatoide Arthritis, M. Parkinson, Hirninfarkt, Dupuytren Kontraktur und ein complex regional pain syndrome aus und diagnostizierten das psychogene "clenched fist syndrom" aufgrund der typischen Handhaltung, dem geringen Leidensdruck, der gelösten Handhaltung im Schlaf und der unauffälligen paraklinischen Befunde. Eine rechtzeitige Diagnosestellung und Mitbetreuung dieser Patienten durch einen Kinder- und Jugendpsychiater ist dringend erforderlich um die potentiell schweren lokalen Komplikationen zu verhindern. 


\section{Varia 2}

\section{DGKJ-P0 64 \\ Eine spezielle Manifestation von CF bei negativem Screeningbefund - Kurzvortrag}

Ludwig Seebauer', Susanne Harner', Uta Duppel², Michael Wurm³, Andrea Schweiger-Kabesch

'Uni-Kinderklinik Regensburg - Standort St. Hedwig, Abteilung für Pädiatrische Pneumologie und Allergologie, Regensburg, Deutschland; ${ }^{2}$ Krankenhaus Barmherzige Brüder - Klinik St. Hedwig Regensburg, Kinder- und Jugendmedizin, Regensburg, Deutschland;'Krankenhaus Barmherzige Brüder Regensburg, Klinik und Poliklinik für Kinder- und Jugendmedizin - Lehrstuhl der Universität Regensburg, Regensburg, Deutschland; ${ }^{4}$ Regensburg, Deutschland

Hintergrund: Das CF Screening bei Neugeborenen wurde in seiner jetzigen Form 09/2016 deutschlandweit eingeführt. Nach mehrfacher Messung von Immunreaktivem Trypsin und Pankreas-assoziiertem Protein werden bei auffälligen Ergebnissen die 31 häufigsten genetischen Mutationen in Deutschland untersucht.

Fallbericht: Es präsentierte sich ein 2 Monate alter Säugling (Geburt 37+4 SSW, GG $3000 \mathrm{~g}(-0.21 \mathrm{z}, 42 \mathrm{P}$.)) mit rez. schwallartigem Erbrechen, ungenügender Gewichtszunahme, entfärbten Stühlen sowie einem intermittierend auftretendem Erythem an Wangen und Windelbereich und Ikterus. Radiologisch bestand ein Mittellappeninfiltrat rechts, Cholestase, Anämie und CMV Infektion. Es zeigte sich eine auffällige Facies mit Pausbacken, sowie grauen Haarsträhnen. Das Aufnahmegewicht lag bei 3650 g (-2,86 $z, 1$. Perzentile), Kopfumfang und Größe ebenfalls unterhalb der 10. Perzentile.

Die Eltern waren türkischer Abstammung und doppelt konsanguin. Ein 10 Jahre alter Cousin der Mutter war an CF erkrankt. Eine genetische Untersuchung zur Familienplanung ergab eine Heterozygotie für die besagte Mutation bei der Schwester der Mutter.

Das initiale Neugeborenen Screening war nicht auswertbar, eine Kontrolle wurde als unauffällig befundet.

Wiederholte Schweißtests ergaben Chloridkonzentrationen von $>80 \mathrm{mmol} / \mathrm{l}$. Das Röntgen-Thorax zeigte infiltrative Veränderungen im Bereich des rechten Mittel- und Unterlappens, sowie mäßige Bronchialwandverdickungen. In der Sonographie war eine erhöhte Echogenität der Leber, bei gleichzeitig nicht darstellbarer Gallenblase zu erkennen Eine Gallengangsatresie konnte in der HBSS sowie bioptisch ausgeschlossen werden. Die Genetik ergab, dass die Patientin Homozygot für CFTR $[$ c.1116+1G >A] (Homozygote Variante ACMG5) ist.

Bei Exokriner Pankreasinsuffizienz erfolgte die Therapie mit Nortase. Die Lungenbeteiligung wurde antibiotisch sowie mit Inhalationen therapiert Die Vitamine und Spurenelemente (V.a. Zink und Selen) bedarfsgerecht substituiert. Die Muttermilchernährung um Cystilac ergänzt. Der CMV mit Valganciclovir behandelt. Bei Anämie insgesamt 3malige Transfusion. Hierunter kam es zu einer deutlichen Verbesserung des Allgemeinzustandes, sowie zu einer raschen Gewichtszunahme.

Schlussfolgerung: 1 . Im diesem Fall zeigt sich, eine aussergewöhnliche Manifestation der CF mit Hautveränderungen, Doll-like Face („puffy cheeks") und auffällig grauem Haarbüschel, wie es bereits bei Buket et. Al 2011 beschrieben wurde. Der Fall deckt auf, wie schwierig eine genetische Kinderwunschberatung bei konsanguinen Eltern sein kann, die aus einem anderen Kulturkreis stammen. Diskutieren lässt sich weiter, ob bei dieser speziellen Form der Mukoviszidose nicht auch der ausgeprägte Nährstoffmangel ursächlich für die sich dargestellte Panzytopenie war, oder zumindest für eine verzögerte Regeneration bei bestehender CMV Infektion gesorgt hat. Trotz Screening muss an durch seltenere Mutationen verursachte CF bei klinischer Symptomatik gedacht werden.
DGKJ-PO 65

Impfraten und Impfzeitpunkte der PneumokokkenKonjugatimpfung bei reifgeborenen Säuglingen vor und nach Umstellung auf das $\mathbf{2}+\mathbf{1}$ Schema

Maren Laurenz', Christof von Eiff ${ }^{2}$, Kathrin Borchert ${ }^{3}$, Sebastian Braun ${ }^{3}$, Christian Jacob ${ }^{3}$, Julia Schiffner-Rohe ${ }^{4}$, Katharina Schley ${ }^{4}$

${ }^{1}$ Pfizer Pharma GmbH, Pfizer Vaccines, Berlin, Deutschland;'2Pfizer Pharma $\mathrm{GmbH}$, Vaccines, Berlin, Deutschland; ${ }^{3} \mathrm{Xcenda} \mathrm{GmbH}$, Real World Evidence, Hannover, Deutschland; ${ }^{4}$ fizer Deutschland $\mathrm{GmbH}$, Health Technology Assessment \& Outcomes Research, Berlin, Deutschland

Hintergrund: Die ständige Impfkommission (STIKO) empfahl bis August 2015 für alle Kinder bis zu 24 Monaten eine Pneumokokken-Konjugatimpfung im sogenannten 3+1 Schema, mit Impfungen im Alter von 2, 3, 4 und 11-14 Lebensmonaten (LM). Im August 2015 wurde das empfohlene Impfschema für alle reifgeborenen Kinder um eine Dosis reduziert (2+ 1 Schema mit Impfungen im Alter von 2, 4 und 11-14 LM). Die Rationale hierfür war, dass trotz einer möglicherweise geringeren Effektivität des $2+1$ Schemas den Kindern eine Impfung weniger verabreicht werden muss, assoziiert mit Kosteneinsparungen und einer angenommenen besseren Impfakzeptanz.

Fragestellung: Ziel der Studie ist es, Impfraten reifgeborener Kinder der Geburtskohorten 2013 (3+1 Schema empfohlen) und 2016 (2+1 Schema empfohlen) zu ermitteln, sowie die Einhaltung der empfohlenen ImpfZeitfenster zu prüfen und miteinander zu vergleichen. Dargestellt sind die Ergebnisse der Zwischenanalyse der jeweils 1. Quartale der Geburtskohorten 2013 und 2016 mit einer Nachbeobachtung von jeweils 24 LM. Methoden: Retrospektive Datenbankanalyse (InGef, Institut für angewandte Versorgungsforschung Berlin) von Abrechnungsdaten einer für Deutschland bezüglich Alter und Geschlecht repräsentativen Stichprobe mit mehr als 4 Millionen gesetzlich Krankenversicherten. Studienpopulation waren alle Kinder der Stichprobe, die entweder zwischen 01.01.201331.03.2013 oder zwischen 01.01.2016-31.03.2016 reifgeboren und im Beobachtungszeitraum (individuell $24 \mathrm{LM}$ ) durchgängig versichert waren. Kinder mit mind. einer Pneumokokken-Impfung (Impfziffer 89.118 oder 89.120) galten als geimpft. Studienendpunkte waren die Impfrate (Anteil der pro Dosis geimpften Kinder) sowie die Einhaltung der empfohlenen Impfzeiträume.

Ergebnisse: 90,4 \% (91,0 \%, in Klammern jeweils die Daten der Geburtskohorte 2013) der Kinder erhielten bis zum Alter von 24 LM mind. eine Pneumokokken-Impfung, 9,6 \% (9,0 \%) erhielten keine. Eine BoosterImpfung entsprechend dem 3+1 Schema erhielten 66,5\% der in 2013 geborenen Kinder. Eine Booster-Impfung entsprechend dem $2+1$ Schema erhielten 69,8\% der in 2016 geborenen Kinder, zusätzlich erhielten 4,6\% der Kinder dieser Geburtskohorte eine Booster-Impfung nach dem 3+1 Schema. Von den geimpften Kindern erhielten 50,6 \% (42,1 \%) die 1. Dosis gemäß STIKO im Alter von 2 LM. Der Booster im Alter von 11-14 LM erfolgte bei 45,2 \% (46,0 \%) der Kinder zeitgerecht entsprechend der STIKO-Empfehlung.

Schlussfolgerungen: Bei reifgeborenen Kindern ist die STIKO-Empfehlung zum $2+1$ Schema bezüglich der Pneumokokken-Impfung weitgehend umgesetzt worden. Der Anteil der ungeimpften Kinder blieb allerdings konstant, bei der Booster-Impfung im 2. Lebensjahr konnte eine dezente Steigerung der Impfrate beobachtet werden. Beim Anteil der zeitgerecht geimpften Kinder zeigte sich kein substantieller Unterschied, was v. a. auch für die Booster-Impfung gilt. Es gibt bisher keinen Hinweis, dass die Reduktion des Impfschemas zu einer besseren Impfakzeptanz geführt hat. 


\section{DGKJ-PO 66}

\section{Sozialmedizinische Aspekte der ungeordneten Migration von minderjährigen Geflüchteten}

\section{Anne Krieg ', Irmgard Walter', Markus Schubert ${ }^{2}$, Peter Gessler ${ }^{1}$}

'Klinikum Konstanz, Klinik für Kinder und Jugendliche, Konstanz, Deutschland; 'Jugendamt Stadt Konstanz, Jugendamt Stadt Konstanz, Konstanz, Deutschland

Fragestellung: Seit 2017 verzeichnet unsere Klinik aufgrund ihrer geographischen Lage einen sprunghaften Anstieg an unbegleiteten minderjährigen Geflüchteten als Patienten. Ziel dieser monozentrischen retrospektiven Analyse war es, die Auswirkungen dieser neuen Entwicklung $\mathrm{zu}$ analysieren.

Material und Methode: Mittels einer standardisierten Datenbank wurden systematisch Alter, Herkunftsland, Gesundheitsstatus und Vorliegen einer posttraumatischen Belastungsstörung (Diagnose gemäß aktueller Leitlinien) aller in unserer Klinik behandelten Geflüchteten im Zeitraum von März 2017 bis Februar 2019 anonymisiert analysiert.

Ergebnisse: Im Untersuchungszeitraum wurden 95 minderjährige Geflüchtete durch die Bundespolizei im Rahmen des Grenzübertritts in unserer Klinik zur Erstuntersuchung vorgestellt. 90 Patienten waren männlich (94,7 \%). Das Durchschnittsalter lag bei 16,11 Jahren. Die Fluchtdauer betrug durchschnittlich 15,5 Monate. Die Herkunftsländer waren Somalia (32), Gambia (13), Guinea (8), andere afrikanische Länder (19), Afghanis$\tan (4)$, Iran (1) und Albanien (1). Bei 16 Patienten blieb das Herkunftsland aufgrund sprachlicher Barrieren unbekannt.

Bei 12 Patienten (12,6\%) bestand eine aktive Hepatitis B (HBsAntigenNachweis). Bei einem Patienten (1,1\%) wurde eine HIV-Infektion mit hoher Viruslast neu diagnostiziert. Positive MRSA-Abstriche aus dem Rachenraum fanden sich bei 5 Jugendlichen (5,3\%), 10 Patienten (10,5\%) präsentierten sich mit Scabies. Der IGRA-Test war bei 39 Jugendlichen positiv (41,1\%). Hiervon wurden 25 Patienten $(64,1 \%)$ wegen einer aktiven Tuberkulose hier therapiert. Eine posttraumatische Belastungsstörung wurde bei 17 Patienten (17,9\%) diagnostiziert.

Diskussion: Auffallend war der hohe Anteil (76,8 \%) jugendlicher Geflüchteter aus Afrika (73). 67 Jugendliche (70,5\%) präsentierten sich mit akuten oder abgelaufenen Infektionen, welche typisch für die unzureichenden hygienischen Bedingungen während der langen Flucht sind. Entsprechende Infektionsschutzmaßnahmen sind häufig sofort erforderlich. Ein relevanter Patientenanteil zeigte Anzeichen schwerer posttraumatischer Belastungsstörungen. Dringend indizierte Therapien konnte jedoch aufgrund mangelnder Ressourcen nicht in allen Fällen angeboten werden. Eine umgehende Behandlung posttraumatischer Belastungsstörungen ist jedoch unverzichtbar und prognoseentscheidend, sodass hier dringender Handlungsbedarf besteht.

Schlussfolgerung: Das Recht des Kindes auf das erreichbare Höchstmaß an Gesundheit (UN-Kinderrechtskonvention) stellt beim ungeordneten Zustrom minderjähriger Flüchtlingen ein hohes logistisches Problem für das Gesundheitssystem dar, da die notwendigen Strukturen permanent vorgehalten werden müssen. Dies schließt neben entsprechend geschultem medizinischem Personal beispielsweise auch Dolmetscher ein. Die vorliegende Analyse unterstreicht die unzureichende Versorgungsrealität in Deutschland und unterstreicht die dringende Notwendigkeit eines länderübergreifenden politischen Lösung.

\section{DGKJ-PO 67 \\ Clinical symptoms do not reflect functional impairment in early cystic fibrosis lung disease}

Insa Korten', Kathryn Ramsey', Elisabeth Kieninger', Urs Frey2', Ben Spycher ${ }^{3}$, Philipp Latzin', Carmen Casaulta'

'Inselspital, Universitäts Kinderklinik, Pädiatrische Pneumologie, Bern, Switzerland;'²Universitäts-Kinderspital beider Basel (UKBB), Pädiatrische Pneumologie, Basel, Switzerland; ${ }^{3}$ Kinderspital Bern, Institut für Sozial- und Präventionsmedizin, Bern, Switzerland
Background: Lung impairment in cystic fibrosis (CF) can already occur within the first year of life. However, frequency and severity of overt respiratory symptoms in infants with $\mathrm{CF}$ are not known.

Methods: We included 50 infants with CF and 50 healthy matched controls from two prospective birth cohort studies. Respiratory symptoms and respiratory rate were documented weekly throughout infancy. Infants performed lung function measurements within the first weeks of life. Results: The analyses included 4552 data points (2217 in CF). Respiratory symptoms (general, mild or severe) were not more frequent in infants with CF (OR:1.1; 95\% CI: $[0.76,1.59] p=0.6$ ). Early lung function and respiratory rate measurements were elevated in infants with $\mathrm{CF}$ compared to healthy controls, but not associated with respiratory symptoms.

Conclusions: We found no difference in respiratory symptoms between healthy and CF infants. This may indicate that early subclinical changes might not be captured by the clinical presentation of patients.

\section{DGKJ-PO 68}

Ambulante Allgemeine Pädiatrie (AAP) - Herausforderung des ambulanten pädiatrischen Kernversorgungsbereiches mit Blick auf den Masterplan Medizinstudium 2020

\section{Dominik A. Ewald', Ralf Moebus², Folkert Fehr ${ }^{3}$}

1 Praxis Drs. Heuschmann und Ewald, Paediatric Primary Care Centre Regensburg, Regensburg, Deutschland;'Praxis Dr. Moebus, Paediatric Primary Care Centre Bad Homburg vor der Höhe, Bad Homburg vor der Höhe, Deutschland; ${ }^{3}$ Praxis Dr. Fehr und Buschmann, Paediatric Primary Care Centre Sinsheim an der Elsenz, Sinsheim, Deutschland

Einleitung: Der Masterplan Medizinstudium 2020 stellt die universitäre Humanmedizin-Ausbildung vor eine Herausforderung. Ein Viertel der Studierenden soll im Neigungsfach Primärversorgung ausgebildet werden. Der pädiatrische Anteil der ambulanten Primärversorgung beträgt etwa 16,5 \% [B̈̈K 2016]. So müssen ca. 2500 Medizinstudierende in Kinder- und Jugendarztpraxen ausgebildet werden. Wie kann sich die ambulante Pädiatrie auf diese neue Aufgabe in den Praxen vorbereiten? Methodik: Die Deutschen Gesellschaft für Ambulante Allgemeine Pädiatrie, DGAAP, hat auf der 1. Bundeskonferenz zur Ausbildung in der Ambulanten Allgemeinen Pädiatrie (AAP) am 10.04.2019 in Frankfurt a. M. die 16 Ausbildungsbeauftragten der Landesverbände des Berufsverbandes der Kinder- und Jugendärzte Deutschlands, BVKJ, eingeladen. In Gruppenarbeiten wurden die Themen Integration der AAP in die universitäre Ausbildung, Kinder- und Jugendarztpraxen als Lernorte der medizinischen Ausbildung und Lerninhalte der ambulanten allgemeinen Pädiatrie erarbeitet und mittels SWOT-Analyse Stärken, Schwächen, Chancen und Risiken eingeschätzt und diskutiert.

Ergebnis: Ambulante allgemeine Kinder- und Jugendmedizin soll von niedergelassenen Allgemeinpädiatern unterrichtet werden. Die Integration der AAP in die universitäre Medizin soll mit Lehrbeauftragten und - ähnlich wie die Entwicklung in der Allgemeinmedizin vor 25 Jahren begann auch mit eigenen Lehrstühlen erfolgen. Diese Lehrstühle sollen Forschung und Lehre in der AAP vertreten und leisten. Die Kooperation mit den bestehenden pädiatrischen Lehrstühlen ist unverzichtbar. Neue Konzepte in Lehre und Forschung müssen erprobt werden.

Studierende sollen da ausgebildet werden, wo Kinder ambulant behandelt werden. Spezielle Praxen müssen Lehrpraxen werden und organisatorische und räumliche Bedingungen erfüllen. Das Personal muss für den Umgang mit Studierenden geschult und eine Ansprechperson zu definierten Zeiten verfügbar sein. Die lehrenden Pädiater in den Praxen werden „Train the Trainer"-Prozesse durchlaufen, unterliegen einer kontinuierlichen Weiterqualifizierung und akademische Prüfungen abnehmen.

Studierende sollen gesunde von kranken Kindern unterscheiden und häufige ambulante Vorstellungsgründe kennenlernen. Die Lerninhalte werden anhand eines einheitlichen Gegenstandkataloges vorgegeben. Neue Medien und Apps machen es möglich, abgeleistete Lehrinhalte zu dokumentieren und Studiengänge flexibel zu gestalten.

Diskussion: Der Masterplan 2020 macht eine Öffnung der universitären Pädiatrie in die ambulante Versorgung notwendig. Niedergelassene Pä- 
diater sind bereit, Teile der ambulanten Ausbildung zu leisten und ihre Expertise in Lehre und Forschung - auch mit Blick auf eine qualifizierte Versorgung in der Zukunft - einzubringen. Der Dialog innerhalb der Universitäten und mit Vertretern der ambulanten Medizin scheint je früher desto besser notwendig, um konstruktiv die gesetzlichen Vorgaben mit allen Betroffenen erfolgreich umzusetzen.

\section{DGKJ-PO 69}

Weiterbildung in Ambulanter Allgemeinpädiatrie: Konstruktion, Einführung und Wirkung des elektronischen Logbuches www. paedcompenda.de

Folkert Fehr', Wolfgang Gempp ${ }^{2}$, Christoph Weiß-Becker ${ }^{3}$, Ulrike Gitmans ${ }^{4}$, Elke Jäger-Roman ${ }^{5}$

${ }^{1}$ Gemeinschaftspraxis, für Kinder- und Jugendmedizin, Sinsheim, Deutschland; ${ }^{2}$ Deutsche Gesellschaft für Ambulante Allgemeine Pädiatrie,

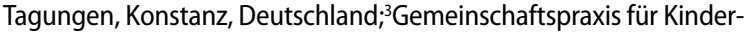
und Jugendmedizin Dr. Becker \& Dr. Weiß-Becker \& Müller-Breitkreuz, Ambulante Allgemeinpädiatrie, Husum, Deutschland; ${ }^{4}$ Gemeinschaftspraxis Dr. med. Ulrike Gitmans, Jan-Peter Schubert, Claudia Laudeley, Dr. med. Anke von Garrel, Ambulante Allgemeinpädiatrie, Saterland - Ramsloh, Deutschland; ${ }^{5}$ Deutsche Gesellschaft für Ambulante Allgemeine Pädiatrie, Strategie, Berlin, Deutschland

Hintergrund: Die Weiterbildung in Deutschland ist im Umbruch. Traditionell klafft ein tiefer Graben zwischen der Ausbildung, die universitätsbasiert, naturwissenschaftlich und mit vielen multiple-choice Fragen aufgestellt ist und der Weiterbildung, die krankenhausbasiert, arbeitsbegleitend und praktisch organisiert ist. Für eine strukturierte kompetenzbasierte Weiterbildung fehlt eine flächendeckende, verbindliche curriculare Vorgabe und damit die Grundlage der Vergleichbarkeit der Kompetenzen von Ärztinnen in Weiterbildung (ÄiW).

Zielsetzung: Phasen während der medizinischen Weiterbildung, die der Grundversorgung gewidmet sind, sollten in jede pädiatrische Weiterbildung einbezogen werden. Pädiatrische Grundversorgung muss die Orte an denen eine solche Ausbildung stattfindet, zu Lernumgebungen entwickeln. Nur dort können Weiterzubildende praktische im Sektor Primärversorgung handeln. Die praktizierenden Fachkräfte werden zu Mentoren Methoden: Die Arbeitsgruppe Weiterbildung der Deutschen Gesellschaft für Ambulante Allgemeine Pädiatrie (DGAAP) hat deshalb nach dem Konzept der Entrustable Professional Activities reale anvertraubare professionelle Tätigkeiten (APT) der Grundversorgung identifiziert, über Anzahl und Umfang entschieden, Titel und Inhalte definiert. Kompetenzdomänen wurden ausgewählt, erforderliches Wissen und notwendige Fähigkeiten spezifiziert und Prüfungsmethoden beschrieben. Über 200 ÄiW und Weiterbilder nutzen das Online-Tool www.paedcompenda.de. Ergebnisse: 12 APTs werden bundesweit genutzt. Die ÄiW bearbeiten die Lernzielkataloge in Rücksprache mit den Weiterbildern, die Weiterbilder dokumentieren Ihre Beobachtungen von Arzt-Patienten-Kontakten (Rückmeldebegegnung) in Rücksprache mit den ÄiW. Eine Matrix stellt sicher, dass alle Teilkompetenzen des Lehrplans abgedeckt werden. Untersuchungen zeigen Vorkommen, Häufigkeit und Lerngeschwindigkeit der einzelnen Teilkompetenzen.

Fazit: Die Einführung der kompetenzbasierten Weiterbildung in der Pädiatrischen Grundversorgung wurde mit Begeisterung und Vorbehalten aufgenommen. Befürworter sehen eine Neuausrichtung auf gegenseitiges Vertrauen im Netzwerk von ärztlichen und nicht-ärztlichen Mentoren, ÄiW, Kindern und Familien sowie für die Gesellschaft insgesamt. Kritiker fürchten einen erheblichen Mehraufwand an Arbeit, Bürokratie und ein Abwandern gefragter ÄiW aus Krankenhäusern in die Grundversorgung. Entrustable Professional Activities - Visualization of Competencies in Postgraduate Training.

\section{Literatur}

1. Position Paper of the Committee on Postgraduate Medical Training of the German Society for Medical Education (GMA). Berberat PO, Harendza S, Kadmon M, GMS Z Med

\section{DGKJ-PO 70}

\section{Simulationstrainings erhöhen die subjektiv empfundene} Sicherheit bei pädiatrischen Notfällen

\section{Anthea Peters', Johannes Breuer', Dejan Vlajnic ${ }^{3}$, Sven Dannemann}

${ }^{1}$ Unikinderklinik Bonn, Kardiologie, Bonn, Deutschland;'2Universitätskin derklinik Bonn, Kinderkardiologie, Bonn, Deutschland;'Marienhospital Bonn, Allgemeinpädiatrie, Bonn, Deutschland; ${ }^{4}$ Marienhospital Bonn, Neonatologie, Bonn, Deutschland

Hintergrund: Pädiatrische Notfälle sind seltene Ereignisse, die mit einem hohen Stresslevel für das behandelnde Team verbunden sind. In Simulationskursen werden Algorithmen trainiert sowie fachliches Wissen und Teamkompetenzen vermittelt. Ein häufig genannter Grund für den Besuch eines Reanimationskurs ist die subjektiv empfundene Unsicherheit der Teilnehmer. Unsicherheit und Angst führen nicht nur zu einem „schlechten Gefühl“; sie gehen einher mit einem erhöhten Stresslevel und schlechterer Performance im Notfall.

Fragestellung: Kann durch ein Simulationstraining die subjektiv empfundene Sicherheit der Teilnehmer verbessert werden? In welchen Teilbereichen der Notfallbehandlung gelingt dies besser oder schlechter?

Material und Methoden: In sieben zweitägigen PALS (Pediatric Advanced Life Support)-Simulationskursen wurden insgesamt 73 Teilnehmer vor und nach dem Kurs nach ihrer subjektiv empfundenen Sicherheit bei pädiatrischen Notfällen befragt. Dabei wurden die Teilbereiche Medikamentendosierung, Teamführung, Angehörigenarbeit, pädiatrische Krankheitsbilder und Reanimationsalgorithmen erfasst. Die Einschätzung erfolgte auf einer Skala von 1-10 ( $1=$ sehr unsicher, $10=$ sehr sicher $)$.

Ergebnisse: Im Rahmen der PALS-Kurse kam es zu einem statistisch signifikanten Anstieg der subjektiv empfundenen Sicherheit um durchschnittlich 2,5 Punkte. Die insgesamte Sicherheit bei pädiatrischen Notfällen stieg von 4,2 Punkten auf 7 Punkte $(+2,8)$. Am besten bewerteten die Teilnehmer ihre Sicherheit bezüglich der pädiatrischen Reanimationsalgorithmen (5,8 vor, 8,1 Punkte nach dem Training). Die größte Unsicherheit bestand im Bereich der Medikamentendosierung (3,8 und 6,5 Punkte).

Diskussion: In zahlreichen Studien zeigt sich ein positiver Aspekt von Simulationstrainings sowohl auf die Performance der Teilnehmer in Testszenarien als auch auf die Überlebensrate von pädiatrischen Patienten. Ursache für die positiven Effekte sind sicherlich ein Zugewinn an praktischen Fähigkeiten und Training in Bezug auf fachliche Aspekte und Teamdynamik. Zusätzlich zeigen Studien, dass es durch eine zunehmende Selbstsicherheit der Teilnehmer zu einem Absinken des Stresslevels in Simulationssituationen kommt. Daher lässt sich postulieren, dass ein Teil des positiven Effekts durch eine zunehmende Sicherheit und damit herabgesetztes Stresslevel der Teilnehmer zustande kommt. Unsere Untersuchung zeigt, dass durch das PALS-Simulationstraining in sämtlichen Aspekten der Behandlung pädiatrischer Notfälle eine Verbesserung der subjektiven Sicherheit erreicht wurde. Weitere Studien sind notwendig um zu zeigen, inwieweit die Sicherheit mit der tatsächlichen Performance und dem Stressniveau in realen Notfallsituationen korreliert und in welchen Abständen Trainings notwendig sind, um die erworbene Sicherheit aufrecht zu erhalten. Insgesamt ist zu fordern, regelmäßiges Simulationstraining mit Personal von Kinderintensivstationen und - notaufnahmen durchzuführen.

\section{Literatur}

1. Judd BK, Currie J, Dodds KL, Fethney J (2019) Gordon C Registered nurses psychophysiological stress and confidence during high-fidelity emergency simulation: Effects on performance. Nurse Educ Today 29(78):44-49. https://doi.org/10.1016/j. nedt.2019.04.005 


\section{DGKJ-PO 71}

\section{Ein außergewöhnlich schwerer Verlauf eines Kawasaki-Syndroms mit intestinaler Beteiligung}

Christine Müller', Florian Schneider², Laura Marchena', Thomas Frank ${ }^{3}$, Meike Franssen ${ }^{4}$, Katharina Knop ${ }^{7}$, Florian Urlichs ${ }^{5}$, Michael Böswald ${ }^{1}$

1 'St. Franziskus Hospital Münster, Allgemeine Kinder- und Jugendmedizin, Münster, Deutschland; ${ }^{2}$ St. Franziskus Hospital, Klinik für Pädiatrie und Neonatologie, Münster, Deutschland; 3 St. Franziskus Hospital Münster, Klinik für Neonatologie und Kinderkardiologie, Münster, Deutschland; ${ }^{4}$ St. Franziskus Hospital Münster, Klinik für Neonatologie, Münster, Deutschland; 5 St. Franziskus Hospital Münster, Klinik für Neonatologie, Münster, Deutschland

Hintergrund: Das Kawasaki-Syndrom ist eine systemische Vaskulitis der mittleren Gefäße. Begleitende intestinale Symptome sind beschrieben, aber selten schwerwiegend. Hier wird ein Patient mit Kawasaki-Syndrom und lebensbedrohlicher duodenaler Ulkusblutung und Invagination beschrieben.

Fallbericht: Ein 4-jähriger Junge wurde mit Erbrechen und Durchfall, Fieber seit vier Tagen, makulopapulösem Exanthem, trockenen Lippen und bilateraler Konjunktivitis aufgenommen. Das Aufnahmelabor zeigte: $\mathrm{CrP}$ 105 mg/l, 8,6 g/dl, Leukozyten 6,1/nl, Thrombozyten 89/nl und Serumnatrium $126 \mathrm{mmol} / \mathrm{l}$. Sonographisch: paralytischer Ileus. Antibiotische Therapie über 3 Tage ohne Besserung, dann Lacklippen, Enanthem, makulopapulöses Exanthem stammbetont, Lymphadenitis colli, Konjunktivitis und Erweiterung der Koronargefäße (LCA 3,6 mm). Diagnose eines Kawasaki-Syndroms. Die zweimalige Immunglobulin-Gabe $(4 \mathrm{~g} / \mathrm{kg})$ und ASS $(60 \mathrm{mg} / \mathrm{kg} / \mathrm{d})$ zeigten eine unzureichende Besserung. Am 6. Tag zeigte sich ein $\mathrm{Hb}$-Abfall $(5,0 \mathrm{~g} / \mathrm{dl})$. In der ÖGD wurde ein blutendes Ulcus duodeni gefunden. Versorgung mit Adrenalin und Fibrinkleber. Die Therapie mit ASS wurde vorübergehend pausiert. Bei Verdacht auf sekundäre Makrophagenaktivierung (Hyperferritinämie, Hypertriglyzeridämie, erhöhte LDH und Transaminasen, persistierend aktivierte Gerinnung mit erniedrigtem Fibrinogen) gaben wir Methylprednisolon $(30 \mathrm{mg} / \mathrm{kg} / \mathrm{d}$ für 3 Tage). Im Tagesverlauf kam es zu einer Schock-Symptomatik, die mehrere Transfusionen und eine Katecholamintherapie erforderte. Ursächlich zeigte sich endoskopisch eine spritzende arterielle Blutung aus vorbestehendem Ulkus. Offen chirurgisch konnte das tiefwandig perforierte Ulcus reseziert werden. Histologisch zeigte sich eine vaskulitisassoziierte ischämische Nekrose. Im Verlauf gelang ein Kostaufbau nur protrahiert. Im MRT-Abdomen fand sich eine ileo-ileale Invagination. Operativ gelang eine Desinvagination problemlos. Postoperativ gelang ein zügiger Kostaufbau, echokardiographisch zeigten sich eine Rückbildung der Koronarerweiterung und es erfolgte die Entlassung nach 35 Tagen.

Schlussfolgerung: Unser Fallbericht beschreibt eine schwerwiegende intestinale Beteiligung beim Kawasaki-Syndrom. Er stellt die erschwerte Diagnosestellung dar, da eine abdominelle Symptomatik der Erfüllung der klassischen Kriterien vorausgehen kann, unterstreicht aber gleichzeitig die Bedeutung einer frühen Diagnose und Therapie. Patienten mit relevanter abdomineller Beteiligung werden in der Literatur als besondere Risikogruppe angesehen, ein Versagen der Immunglobuline sowie ein höheres Risiko für Aneurysmen der Koronarien sind beschrieben.

\section{DGKJ-PO 72}

Subtile Anamneseerhebung nicht vergessen! Zum weiterhin hohen Stellenwert in der Medizin trotz zunehmender "Gerätemedizin“ und Gefahr von Überdiagnostik Fallbericht einer Patientin mit gravierenden rezidivierenden Bauchbeschwerden

\section{Richard Eyermann ${ }^{1,2}$}

'Dr. Eyermann München, Kinder- und Jugendmedizin, Kinderkardiologie, Sportmedizin, München, Deutschland; '²linik Schönsicht Berchtesgaden, Rehabilitation für Kinder und Jugendliche, AHB, Kind-Mutter/VaterRehabilitation, München, Deutschland
Problemstellung: In Medizinzeiten mit Diagnosestellung durch immer aufwändigere Bildgebung und umfangreiches Labor wird v.a. durch jüngere Ärzte die Anamnese mit klinischem Status praesens mit daraus resultierender Verdachtsdiagnose und Differenzialdiagnosen zunehmend unterschätzt.

Fallbericht: 12 6/12 Jahre altes Mädchen zur Rehabilitation bei V.a. psychosomatischen Beschwerden. Seit 3 Jahren rezidivierende Bauchschmerzen, plötzlich einsetzend und teilweise sehr stark. Umfangreiche kindergastroenterologische Diagnostik erfolgt:

Atemtest auf H.p.v. 11/2015: Test: Infai Delta-Wert 30 min nach $75 \mathrm{mg}$ 13C-Harnstoffgabe: 0,1

Test: Diabact Delta-Wert 10 min nach 50 mg 13C-Harnstoffgabe: $0,1(<2,5$ bzw. $<2,2$ kein Hinweis auf H. p.-Befall);

Gastroskopie + PEs v. 7/2016: Endoskopie des oberen GI-Traktes makroskopisch unauffällig. Hinweise auf H.p.-Infektion weder mikrobiologisch noch im Schnelltest.

Großes Labor v. 1/2017 und 1/2018: insges. unauffällig.

Allergietestung: Rast v. 1/2017: Nahrunsgmittelscreen FX5: negativ; Rast v. 1/2017: Phadiatop Screen SX1; negativ; Ges.-IgE(S): 48,10 U/ml (NW $<120)$

H2-Exhalationstests: Lactose v. 11/2016: H2 ppm 0-120 min: 17,14,10,9,9; kein max. Anstieg, keine Beschwerden, Normalbefund; Fructose v. 1/2017: H2 ppm 0-90 min: 2,3,12,14; max. Anstieg 12 ppm, keine Beschwerden, Normalbefund.

Bei Annahme psychosomatischer Beschwerden, Verlegenheitsdiagnose rez. Gastritis und probatorische PPI (Omeprazol)-Therapie.

Bei Aufnahme in der Reha-Klinik und subtiler Anamneseerhebung berichtete die Mutter über die Assoziation mit ausgedehnter Urticaria jeweils bei Bauchschmerzen. Viele Handy-Fotos der Mutter lagen vor, wurden aber ärztlicherseits nicht beachtet.

Diskussion und Konklusion: Unabhängig von der Verlaufsform der Nesselsucht zeigen alle Formen die gleichen Symptome, nämlich das plötzliche Auftreten von Juckreiz, Rötungen und Quaddeln oder Schwellungen der Haut (Angioödeme).

Zusätzliche Beschwerden, die bei der Urtikaria auftreten können, sind Schluckbeschwerden, Atemnot, Bauchschmerzen, Übelkeit und Durchfall. Dies lässt sich durch die Tatsache erklären, dass die Mastzellen nicht nur in der Haut, sondern auch an den Schleimhäuten der Atemwege und des Magen-Darm-Traktes vorkommen.

Eine Urticaria ist in den wenigsten Fällen $(<5 \%)$ allergisch bedingt, wird aber oft als Ursache vermutet! Häufigste Ursachen sind Infektionen, physikalisch (Wärme, Kälte, mechanisch), Begleitsymptom bei immunologischen Erkrankungen, in ca. $50 \%$ unklar.

Bei Urticaria können Bauchschmerzen auftreten. Eine subtile Anamneseerhebung kann Überdiagnostik verhindern und psychosomatische Erklärungsversuche ausräumen.

\section{DGKJ-PO 73}

\section{It is not easy to find the right diagnosis: Cystic teratoma in a 3 year} old patient

Florian Schneider', Florian Beyer', Lars Grüber ${ }^{3}$, Jean Tsokas ${ }^{3}$, Ludger Heuckmann ${ }^{4}$, Michael Böswald'

'St. Franziskus Hospital, Allgemeine Kinder- und Jugendmedizin, Münster, Germany; ${ }^{2}$ St. Franziskus Hospital, Klinik für Radiologie, Münster, Germany; ${ }^{3}$ St. Franziskus Hospital Münster, Klinik für Kinderchirurgie, Münster, Germany; ${ }^{4}$ Kinderarztpraxis Becker/Heuckmann, Kinderarztpraxis Becker/Heuckmann, Münster, Germany

Question: A 3 year old boy with an extended belly visited the pediatric surgery department of our hospital with an unclear abdominal mass below the left rib cage. The boy presented in good health, normal vital signs, appropriate growth percentiles according to his age and gender except an extended belly. He had no pain on palpation, no problems with passing stool or urine and no other pathologies like night sweats, weight loss or other signs of being unwell. Furthermore, the laboratory did not show any abnormalities. 
Material \& method: Ultrasound revealed an abdominal mass consisting of colonic structures with wide lumen (max. $3,5 \mathrm{~cm}$ ) and a trilaminar appearance of the wall. Moreover, an elongation of the sigmoid colon was seen suggesting megacolon or constipation. Even though multiple laxative procedures were induced, the results of the ultrasound stayed the same. Using contrast enema to rule out chronic Hirschsprung's disease, there were signs of displacement of the colonic frame and the small bowels to the periphery suggesting a space occupying mesenteric mass.

For further investigation an MRI scan was performed. A multi cystic abdominal mass $(16 \mathrm{~cm} \mathrm{x} \mathrm{8,5} \mathrm{cm} \times 17 \mathrm{~cm})$ was seen in the upper abdomen. Different compartments within the mass were divided by septs with slight linear contrast enhancement. The differential diagnosis at this point consisted of mesenteric lymphatic malformation (i. e. lymphangioma) or (multiple) gastrointestinal duplication cysts. Other visceral organs did appear normal in size and shape without infiltration.

Results: The boy underwent explorative laparotomy in May 2018 without any complications. The tumor weighed $1.8 \mathrm{~kg}$ and had components of bone and cartilage, suggesting being a teratoma. It was embedded in peritoneum, mesenterium and omentum. It reached from the diaphragm to the pelvis minor. There was no infiltration in other organs.

The histological examination resulted in a cystic teratoma. It consisted of epithelial, neuro-ectodermal and mesenchymal parts. The wall of the teratoma had colon-like structures with intramuscular and submucous ganglion cells, which can explain the motility within the mass, previously seen in the ultrasound examination as peristalsis. There were no signs of any malignancy within the tumor and the borders of the resected tumor were free of any malignancies as were the resected lymph nodes. Since the surgeons palpated bone like structures within the tumor an x-ray examination of the teratoma was conducted, which showed bones and cartilages in no specific order.

Conclusion: In outpatient departments pediatricians often examine extended bellies in children. The most likely diagnoses are constipation or meteorism. Tumors of soft tissues in children and adolescents, however, are scarce (1/100.000 children/year) and clinically difficult to diagnose. We presented a rare case causing an extended belly in a 3 year old patient. In a multi-discplinary team we could quickly diagnose and treat it. 


\title{
Abstracts der 71. Jahrestagung der Deutschen Gesellschaft für Sozialpädiatrie und Jugendmedizin (DGSPJ)
}

\author{
Wissenschaftliche Leitung: \\ Dr. Andreas Oberle
}

\section{Freie Vorträge}

\author{
Versorgungsforschung für Kinder und Jugendliche - geförderte \\ Projekte aus dem Innovationsfonds \\ Silke Pawils \\ UniversitätsklinikumHamburg-Eppendorf, Institut f. Med. Psychologie, \\ Forschungsgruppe Prävention im Kindes- und Jugendalter, Hamburg, \\ Deutschland
}

Psychosoziale Grundversorgung im Ambulanten Sektor

- Schnittstellen- und Datenschutzmangement in der psychosozialen Versorgungsforschung

Hintergrund: Das Innovationsfondprojekt „KID-PROTEKT“ untersucht, wie die systemübergreifende Zusammenarbeit von ambulanter Gynäkologie bzw. Pädiatrie mit der Kinder- und Jugendhilfe zur Umsetzung der gesetzlich geforderten Präventionsleistungen unter Effektivitäts- und Effizienzaspekten bestmöglich gelingen kann. Ziel ist die Förderung einer gesunden Kindesentwicklung trotz psychosozialer Belastungssituation in der Familie durch „Schnittstellenmanagement“ in der Arztpraxis. Die bestehende Regelversorgung zur Schwangerenvorsorge bzw. Früherkennung von Krankheiten bei Kindern und Jugendlichen wird um eine systematische psychosoziale Belastungsanamnese erweitert und führt im Bedarfsfall zu einer Überleitung ins Jugendhilfesystem.

Fragestellung: Verschiedene Sozialrechtsbereiche (Gesundheits- und Jugendhilfesystem) müssen methodisch erfasst und in die Evaluation der Interventionen einbezogen werden unter der Berücksichtigung relevanter Datenschutzrichtlinien. Wie kann dies bei einem RCT-Forschungsdesign gelingen und gleichzeitig auch Aussagen der eigentlichen Zielgruppe einbezogen werden bzw. eine Nicht-Teilnehmer durchgeführt werden kann. Methodik: In einem clusterrandomisierten Kontrollgruppenvergleich gegenüber „Treatment as usual“ (TAU) wird ein Verfahren, bei dem eine sozialpädagogische Fachkraft eine Sprechstunde in der Praxis durchführt und die Lotsenfunktion zu den Hilfsangeboten übernimmt („Supported Treatment") mit einer Variante ohne Lotsin („Qualified Treatment“) verglichen. Die teilnehmenden Frauen- und Kinder- und Jugendarztpraxen werden randomisiert den Bedingungen zugeordnet und der psychosoziale Unterstützungsbedarf der Patienten erhoben. Primäre Outcomes sind Effektivität und Effizienz im Vergleich zur aktuellen Versorgung. Dazu werden die Bedarfsquote im Verhältnis zur Belastungsquote, die Weiterleitungsquote im Verhältnis zur Bedarfsquote und die Häufigkeit der Inanspruchnahme gemessen. Als sekundäre Outcomes werden Akzeptanz und Machbarkeit mittels Patienten- und Praxenbefragung ermittelt und die Generalisierbarkeit u. a. durch Analyse regionaler Einflüsse geprüft. Eine Nicht-Teilnehmer-Analyse gibt Aufschluss über die Gründe der Teilnahmeverweigerung zu unterschiedlichen Zeitpunkten der Versorgung.

Ergebnisse: Die Problematik der Evaluation des Schnittstellenmanagements wird erläutert und eine Lösung aufgezeigt. Das Datenschutzkonzept des Projekts wird dargestellt und die Besonderheiten zur Diskussion gestellt.
Interessant und wichtig

\author{
DGSPJ-FV 01 \\ SINDA: Eine neue standardisierte entwicklungsneurologische \\ Screening-Untersuchung für das Säuglingsalter. Gütekriterien der \\ Neurologischen Skala. \\ Uta Hedwig Tacke' ${ }^{1}$ Heike Philippi' ${ }^{2}$ Joachim Pietz ${ }^{3}$, Rupp André4, Mijna \\ Hadders-Algra ${ }^{5}$ \\ 'Universitäts-Kinderspital beider Basel (UKBB), Neuro-Onkologie, Basel, \\ Schweiz; ${ }^{2}$ Sozialpädiatrisches Zentrum Frankfurt Mitte, SPZ, Frankfurt am \\ Main, Deutschland; ${ }^{3}$ Kinder Palliative Team Südhessen, Palliative Medizin, \\ Frankfurt am Main, Deutschland; ${ }^{4}$ Universitätsklinikum Heidelberg, Sektion \\ Biomagnetismus, Heidelberg, Deutschland; ${ }^{5}$ Universität Medical Center \\ Groningen, Entwicklungsneurologie, Groningen, Niederlande
}

Fragestellung: Die entwicklungsneurologische Beurteilung von Säuglingen dient zwei Zielen: 1. Der Feststellung des aktuellen zur Identifikation von Förderbedarf und Beratung der Eltern, 2. der Prognoseabschätzung, insbesondere bei Kindern mit bekannten Entwicklungsrisiken. Das „Standardized Infant NeuroDevelopmental Assessment (SINDA)“ ist ein neues Screeningverfahren für das korrigierte Alter (KA) von 6 Wochen bis zu 12 Monaten. SINDA besteht aus drei Skalen: der Neurologischen Skala, der Entwicklungs-Skala und der Sozio-Emotionalen Skala. Hier wird die Neurologische Skala, das neurologische Untersuchungsprotokoll vorgestellt. Sie umfasst 28 altersunabhängige Items aus den fünf Domänen Spontanmotorik, Hirnnerven, Motorische Reaktionen, Tonus und Reflexe. Die Gesamtpunktzahl ergibt sich aus der Summe der bestandenen Items, mit einem Maximalwert von 28. Die Durchführungsdauer beträgt etwa 10 min. Kollektiv und Methode: Insgesamt 181 Säuglinge ( $83 \%$ ehemalige Frühgeborene) wurden im korrigierten Alter (KA) von 6 Wochen bis 12 Monaten im SPZ Frankfurt Mitte mit der SINDA Neurologischen Skala untersucht und mit $\geq 24$ Monaten (24-41 Monate) KA nachuntersucht. Zur Bestimmung der Interrater-Reliabilität beurteilten drei Autoren zweimal unabhängig voneinander insgesamt 24 Untersuchungsvideos. Die SINDA Vorhersagevalidität wurde an den Ergebnissen der Nachuntersuchung mit $\geq 24$ Monaten KA geprüft. Dazu gehörten der neurologische Befund und die Ergebnisse eines standardisierten Untersuchungsverfahrens, meist den Bayley Scales of Infant Development II (BSID II).

Ergebnisse: 1. Reliabilität: Sowohl für das SINDA-Gesamtergebnis als auch für die einzelnen Domänenpunkte ergab sich eine sehr hohe Intra- und Interrater-Reliabiltät (Intraclass Correlation Coeffizient 0,923-0,965). 2. Vorhersagevalidität: Insgesamt 56 Kinder (31 \%) hatten im Alter von $\geq 24$ Monaten Entwicklungsstörungen, d.h. einen MDI/PDI $<70$ in den BSID II oder eine Cerebralparese nach den klinischen SCPE Kriterien $(n=29)$ neben anderen neurologischen Auffälligkeiten. Ein Gesamtpunktwert $\leq 21$ zeigte eine exzellente Vorhersagevalidität für das Vorliegen einer Cerebralparese (Sensitivität 1,0, Spezifität 0,81 ) oder für einen MDI/PDI $<70$ (Sensitivität: 0,89 , Spezifität: 0,81 ). 
Diskussion und Schlussfolgerung: Die Neurologische Skala von SINDA ist ein schnell durchzuführendes, valides Untersuchungsprotokoll für das erste Lebensjahr. Sie ermöglicht eine neurologische Befunderhebung und erlaubt Entwicklungsprognosen bis ins Kleinkindalter. Als standardisierte Screeningmethode eignet sie sich für die Anwendung in der allgemeinpädiatrischen Praxis, in Kinderkliniken, in Sozialpädiatrischen Zentren und spezialisierten entwicklungsneurologischen Einrichtungen.

\section{Literatur}

1. Hadders-Algra M, Tacke U, Pietz J, Rupp A, Philippi H (2018) Reliability and predictive validity of the Standardized Infant NeuroDevelopmental Assessment neurological scale. Dev Med Child Neurol. https://doi.org/10.1111/dmcn.14045

\section{DGSPJ-FV 02}

Genetische Untersuchungen bei Kindern und Jugendlichen mit Entwicklungsstörungen, Behinderungen und/oder Epilepsien was erwarten Eltern von der Aufklärung

\section{Marie Elisa Mey', Andreas Jenke ${ }^{2}$, Peter Borusiak ${ }^{3}$}

'Universität Witten/Hedecke, Fakultät für Gesundheit, Hamburg, Deutschland; ${ }^{2}$ Klinikum Kassel, Klinik für Neonatologie und allgemeine Pädiatrie, Kassel, Deutschland; ${ }^{3}$ Klinikum Bremen-Mitte, Sozialpädiatrisches Institut, Bremen, Deutschland

Fragestellung: Genetische Untersuchungen haben in den letzten Jahren bei der ätiologischen Abklärung von Kindern mit Intelligenzminderungen, Autismus-Spektrum-Störungen und Epilepsien zunehmend an Bedeutung gewonnen. Das Aufklärungsgespräch für die genetischen Untersuchungen dient den Eltern als Informationsquelle. Dieses sensible Thema stellt jedoch sowohl die Ärzte als auch die Eltern vor eine anspruchsvolle Aufgabe. Der Komplexität der Vorgaben für die Aufklärung steht ein eher geringes Verständnis für Gesundheitsbelange und -zusammenhänge („,health literacy“) gegenüber. Wir haben die Erwartungen von Eltern an eine solche Aufklärung untersucht.

Methode: In einem Mixed-Methods-Ansatz wurden zunächst freie Interviews mit mehreren Ärzten geführt, um relevante Aspekte aus ärztlicher Sicht zu erfassen. Anschließend erfolgte die Entwicklung eines halbstandardisierten Fragebogens anhand ausführlicher, offener und qualitativer Interviews mit 5 Eltern. Der Bogen wurde dann in einer Befragung von 30 Eltern eingesetzt.

Ergebnisse: Die Bereiche „Diagnose“ und „Therapie“ haben bei den befragten Eltern einen hohen Stellenwert. Die Aufklärungsgespräche bedeuten für die Eltern eine Herausforderung und emotionale Anspannung. Die genetischen Vorkenntnisse werden unterschiedlich eingeschätzt, wobei viele Eltern Verständnisproblem während des Aufklärungsgespräches angaben. Überwiegend als „sehr wichtig“ oder „wichtig“ wurden folgende Themenbereiche eingeordnet: Befunde unklarer Zuordnung bzgl. der Pathogenität, Zufallsbefunde, Recht auf Nichtwissen, psychische Folgen, weiterer Verlauf, mögliche Therapien. 10 Eltern hatten entweder keinen oder einen Sonderschulabschluss, 20 Eltern waren im Deutschen Nichtmuttersprachler.

Diskussion: Eltern haben einen hohen Informationsbedarf, der fast alle Bereiche der Aufklärung umfasst.

Die Kommunikation der möglichen Ergebnisse mit unklaren genetischen Varianten und deren Bedeutung, inklusive der Angst und Unsicherheiten der Betroffenen stellt sowohl die Eltern als auch die Ärzte vor eine große Herausforderung. Der Bildungsstand und mögliche sprachliche kommunikative Hürden können zusätzlich die Aufklärung erschweren.

Schlussfolgerung: Es gibt Verbesserungspotential bei der Aufklärung vor molekulargenetischen Untersuchungen. Internet-basierte Angebote in mehreren Sprachen und in leichter Sprache könnten eine Hilfestellung bieten. Gleichzeitig stellt sich die Frage, ob bzw. wie die im Gendiagnostikgesetz aufgeführten und für jeden Arzt verpflichteten Inhalte einer Aufklärung durch die genannten Schwierigkeiten im Alltag umsetzbar sind.

\section{DGSPJ-FV 03 \\ Nicht-akzidentelles Schädeltrauma (NAHT) - klinisches und bildgebendes Spektrum}

\section{Marion Döbler', Ingeborg Krägeloh-Mann ${ }^{2}$}

'Universitätsklinik für Kinder- und Jugendmedizin Tübingen, Neuropädiatrie, Entwicklungsneurologie, SPZ, Tübingen, Deutschland;'ZUniversitätskinder klinik Tübingen, Neuropädiatrie, Entwicklungsneurologie, Sozialpädiatrie, Tübingen, Deutschland

Schädel- und Gehirnverletzungen durch Misshandlung sind bei Säuglingen und Kleinkindern die häufigste nicht natürliche Todesursache. Als wesentlicher Mechanismus wird ein Schütteln eines Kindes angenommen, was zu unkontrolliertem Rotieren des Kopfes führt mit konsekutiver Schädigung des Gehirns in variabler Ausprägung.

Die charakteristische Trias bestehend aus Enzephalopathie, subduralen Hämatomen und retinalen Blutungen findet sich in der Regel beim klassischen, signifikanten Schütteltrauma-Syndrom (STS).

Die klinische Symptomatik kann jedoch variabel und unspezifisch sein, äußere Verletzungszeichen fehlen meist, dies erschwert im Alltag häufig die Diagnosestellung. Hinweisend auf eine nichtakzidentelle Genese sind fehlende oder inadäquate/inkonsistente Angaben der betreuenden Personen.

Material und Methode: Anhand von 4 Fallbeispielen werden das klinische Spektrum von leichten bis schweren Verläufen und die entsprechenden MR-tomographischen Befunde dargestellt.

Ergebnisse: Fall 1: 2 Monate altes Mädchen, Kind wurde im Laufstall schlapp und apathisch aufgefunden. Bei Aufnahme keine Zeichen einer Enzephalopathie. Am 2. stationären Tag fokaler Krampfanfall. In der MR-Bildgebung umschriebenes subdurales Hämatom sowie eine kleine intraparenchymatöse Einblutung. Nachweis disseminierter retinaler Blutungen. Ein STS wird zugegeben. Altersentsprechende Entwicklung im Verlauf

Fall 2: Stationäre Aufnahme des 5 Monate alten Mädchens aufgrund unklarer Hämatome im Halsbereich. Leicht reduzierter AZ, Gedeihstörung, keine akuten neurologischen Symptome. In der MR-Bildgebung subdurales Hämatom und eine umschriebene Parenchymbeteiligung im Marklager. Keine retinalen Blutungen. Altersentsprechende Entwicklung im Verlauf.

Fall 3: Notfallmäßige Aufnahme des 7 Wochen alten komatösen Jungen auf die Intensivstation. In der MR-Bildgebung subdurale Blutungen und gravierende parenchymatöse Verletzungen. Keine retinalen Blutungen. Residualsymptomatik mit geistiger Behinderung, Verhaltensproblematik, Epilepsie und leichter spastischer Hemiparese.

Fall 4: Notfallmäßige Aufnahme des 4,5 Monate alten Jungen auf die Intensivstation bei instabilen Vitalparametern. Anamnestisch Nahrungsverweigerung, bei Trinkversuch sei das Kind plötzlich steif geworden, dann apathisch und schlapp. In der MR-Bildgebung subdurale Hämatome sowie ausgeprägte supra- und infratentorielle corticale Ischämien mit ausgeprägten Substanzdefekten und Hirnatrophie im Verlauf. Ausgedehnte retinale Blutungen. Schwere Residualsymptomatik mit spastischer Tetraparese, Epilepsie, Sehbeeinträchtigung und schwerer globaler Entwicklungsstörung.

Schlußfolgerungen: Die klinisch-neurologische Symptomatik bei NAHT ist variabel und zeigt ein Kontinuum von geringen Funktionsstörungen bis zu schweren akuten Beeinträchtigungen.

Die klassische Trias mit Enzephalopathie, subduralen- und retinalen Blutungen ist nicht immer vorhanden.

Das neurologische Outcome ist vom Ausmaß und der Topographie der Gehirnschädigung abhängig.

\section{Literatur}

1. Herrmann B (2016) Epidemiologie, Klinik und Konzept des Schütteltrauma-Syndroms. Pädiatrische. Praxis 86:297-312

2. Choudhary AK, Servaes S, Slovis TL et al Consensus statement on abusive head trauma in infants and young children. Pediatr Radiol 20: 


\section{DGSPJ-FV 04}

\section{Das Reha $1 \times 1$ - Zugangswege zur (neuro)pädiatrischen Rehabilitation}

\section{Ulf Hustedt}

Helios Klinik Hattingen, Neuropädiatrische Rehabilitation, Hattingen, Deutschland

Fragestellung: Die Anträge zur stationären Rehabilitation im Kindesalter waren in den letzten Jahren rückläufig, was auch an dem aufwändigen und zum Teil unklaren Antragsverfahren lag. Trotz erfreulicher gesetzlicher Änderungen in den letzten Jahren bestehen bei den pädiatrischen Kollegen immer noch große Unsicherheiten hinsichtlich der Beantragung von stationären rehabilitativen Leistungen für Kinder. Dieser Vortrag soll im Sinne eines $1 \times 1$ eine möglichst einfache Hilfestellung bei der Beantragung von stationären rehabilitativen Leistungen am Beispiel der neuropädiatrischen Rehabilitation geben.

Material und Methode: Anhand der letzten gesetzlichen Änderungen wird aufgezeigt, wie eine Antragstellung zur stationären, neuropädiatrischen Rehabilitation ablaufen kann. Von Seiten der Deutschen Rentenversorgung (DRV) wurden dafür Erleichterungen durch das Flexirentengesetz definiert, das zum Dezember 2016 in Kraft getreten ist. Durch die Änderungen im Flexirentengesetz wird die Bedeutung der Kinder-Reha ausdrücklich gestärkt.

Über die Gesetzliche Krankenversicherung (GKV) ist es seit April 2016 für jeden Vertragsarzt möglich, über das neue KV Muster 61, einen RehaAntrag zu stellen. Eine besondere Qualifikation, wie sie zuvor in Form eines Colloquiums notwendig war, muss dafür erfreulicherweise nicht mehr nachgewiesen werden.

Ergebnisse - Diskussion: An Fallbeispielen werden die unterschiedlichen Zugangswege und die möglichen Kostenträger einer stationären rehabilitativen Behandlung diskutiert.

Schlussfolgerung: Die rehabilitative Behandlung hat im Kindesalter eine wichtige, präventive Bedeutung und sollte über die Kostenträger GKV und DRV als stationäre oder ambulante Therapie nicht nur bei akut verunfallten oder operierten Kindern genutzt werden. Auch chronisch kranke und mehrfachbehinderte Kinder profitieren von dieser Form der Behandlung.

\section{DGSPJ-FV 05}

Das Schädel-Hirn-Trauma im Kindesalter: eine chronische Erkrankung?!

Ulf Hustedt

Helios Klinik Hattingen, Neuropädiatrische Rehabilitation, Hattingen, Deutschland

Fragestellung: Das Schädel-Hirn-Trauma gehört zu den häufigsten Unfällen im Kindesalter und kann zu Behinderungen und Todesfällen führen. Fast $30 \%$ aller SHT betreffen Kinder unter 16 Jahren. Glücklicherweise sind weniger als $10 \%$ der SHT im Kindesalter als mittelschwer und schwer einzustufen. Insgesamt ist die Akutversorgung der Patienten in der Pädiatrie, Intensivmedizin, Kinderchirurgie und Neurochirurgie in Deutschland sehr gut. Bleibende Störungen, wie z.B. Paresen, treten für gewöhnlich nur nach einem schweren SHT auf. Die assoziierten knöchernen Verletzungen an den Extremitäten heilen in wenigen Wochen ab. Was häufig bleibt, sind Verhaltensauffälligkeiten, die die Kinder in ihrer weiteren Entwicklung begleiten. Aus neuropädiatrischer Sicht hat das SHT damit den Charakter einer chronischen Erkrankung, was häufig in der weiteren Nachbetreuung der Patienten verkannt oder falsch eingeordnet wird. Material und Methode: Anhand unseres Kollektivs von Kindern und Jugendlichen nach SHT, die zu einer stationären neuropädiatrischen Rehabilitation aufgenommen wurden, werden die Langzeitfolgen des SHT aufgezeigt. Alle Patienten wurden während ihrer Rehabilitation neuropsychologisch betreut und besuchten die Schule für Kranke in unserer Klinik. Unsere Klinik nahm über einen längeren Zeitraum am SHT-Register des Landes Nordrhein-Westfalen teil, auf dessen Erhebungen die Ergebnisse zum Teil gründen. Wichtig ist die Kenntnis von Verhaltens- und Persönlichkeitsveränderungen, die aus einem SHT im Kindesalter resul- tieren können, diese können internalisierend (z. B. Depression, Angst, sozialer Rückzug) oder externalisierend (z. B. hyperaktives oder aggressives Verhalten) sein.

Ergebnisse - Diskussion: Für die neuropädiatrische Rehabilitation konnte gezeigt werden, dass die Patienten deutlich von einer längeren Rehabilitationsdauer profitieren konnten. Bei schweren und mittelschweren SHT beträgt der Anteil von Verhaltensauffälligkeiten nach einem Jahr $66 \%$, selbst bei leichten SHT noch 40 \% (1). Es zeigten sich besonders Probleme im Sozialverhalten, besondere Erregbarkeit, ungewohnte Emotionen und Veränderungen der Persönlichkeit.

Schlussfolgerung: Gilt für den Schlaganfall „time is brain“, so gilt für das SHT im Kindesalter „brain needs time“. Kinder profitieren von einem längeren Rehaaufenthalt, auch noch Jahre nach dem Unfallereignis und zum wiederholten Male (Re-Reha). Entgegen weitläufiger Meinungen trifft eine frühe Schädigung das Gehirn besonders stark, die Langzeitfolgen sind umso gravierender je jünger die Kinder sind. Motorische Defizite bilden sich im ersten halben Jahr zurück (2), neuropsychologische Defizite und psychosoziale Beeinträchtigungen haben langanhaltenden Bestand (3). Darüber sollten die weiterbehandelnden Kollegen informiert sein.

\section{Literatur}

1. Brown G et al. Psychol Med; 1981; 11: 63-78. Rivara JB et al. Arch Phys Med Rehabil 1994; 75: 369-379. Hawley CA. Brain Inj 2003; 17:105-129. Hooper SR et al. NeuroRehabilitation 2004;19:175-189

2. Neuhäuser \& Thyen 2008,601

3. Lehmkuhl et al. 2013,12 


\section{Versorgungsforschung für Kinder und Jugendliche: Geförderte Projekte aus dem Innovationsfonds}

\section{DGSPJ-FV 07 \\ Kinder und Familien stärken für eine gesunde Gewichtsentwicklung: Stufenmodell Adipositas-Therapie im Kindes- und Jugendalter (STARKids)}

Katrin Ziser', Felicitas Stuber', Stephan Zipfel', Stefan Ehehalt'2, Florian Junne 'Universitätsklinikum Tübingen, Psychosomatische Medizin und Psychotherapie, Tübingen, Deutschland; ${ }^{2}$ Universitätsklinikum Tübingen, Psychosomatische Medizin und Psychotherapie, Adipositas-Plattform, Tübingen, Deutschland

Hintergrund: Im Folgenden wird ein vom Innovationsfonds in der Förderlinie neue Versorgungsformen gefördertes Projekt vorgestellt. Die Zielsetzung dieses Projekts ist ein niederschwelliges, strukturiertes und bedarfsgerechtes transsektorales Versorgungsmodell für Kinder und Jugendliche mit Übergewicht und Adipositas zu entwickeln und zu evaluieren, das innovative E-health-Ressourcen integriert zur Verringerung von Übergewicht/Adipositas und der damit verbundenen Folgekrankheiten/Belastungen.

Fragestellung und Ziele: Die neue Versorgungsform wird in einer clusterrandomisierten, kontrollierten Interventionsstudie evaluiert. Die Hauptfragestellungen beziehen sich dabei auf die Verringerung des Gewichts (BMI-SDSLMS), Erhöhung der Lebensqualität, Verbesserung von Folgeerkrankungen von Adipositas, günstige Verläufe bzgl. psycho-bio-sozialer Determinanten und Folgen von Übergewicht und Adipositas (z. B. Motivation, Stigmatisierung, soziale Teilhabe), sowie eine vorteilhafte KostenEffektivitäts-Relation des Interventionsprogrammes.

Material und Methoden: Die neue Versorgungsform gliedert sich in die folgenden Bereiche auf: (i) Entwicklung eines elektronischen Moduls zur strukturierten Anamnese und leitliniengerechten Diagnostik. (ii) Entwicklung eines computergestützten (Tablet-basierten) ambulanten Präsenz-Schulungsprogramms, das in der Kinder- und Jugendarztpraxis (KJAP) gemeinsam mit den betroffenen Familien auf strukturierte, leitlinienbasierte Art und Weise durchgeführt wird. (iii) Konzeption eines unabhängigen, angebotsübergreifenden Fallmanagements durch den öffentlichen Gesundheitsdienst für übergewichtige und adipöse Kinder und Jugendliche mit initial mäßigem/fehlendem Therapieerfolg zur weiterführenden Therapieplanung. (iv) Entwicklung einer Online-Ressource für die Vertiefung der Schulungsinhalte im häuslichen Umfeld mit spielerischer Vermittlung von relevanten Inhalten. Die Interventionsgruppe erhält das dargestellte Interventionsprogramm bestehend aus Präsenzschulungen und Zugriff auf die Online-Ressourcen zur Vertiefung und Reflektion der Inhalte. Die Kontrollgruppe erhält eine einmalige Basisberatung (,Treatment as usual"). Beide Gruppen durchlaufen drei Hauptmesszeitpunkte: $\mathrm{T} 0=$ Baseline, $\mathrm{T} 1=\mathrm{T} 0+12$ Monate und $\mathrm{T} 2=\mathrm{T} 1+6$ Monate .

Ergebnisse und Diskussion: Die Versorgung von Kindern und Jugendlichen mit Übergewicht oder Adipositas soll mit diesem neuen Versorgungsansatz erstmals in einem flächendeckenden, strukturierten, niederschwelligen und nachhaltigen Ansatz ermöglicht werden. Die KJAP wird hierbei in ihrer Rolle als erste und für Familien häufig wichtigste Anlaufstelle in Gesundheitsfragen der Kinder und Jugendlichen gestärkt.

\section{DGSPJ-FV 06}

Stand der sozialräumlichen Hilfe- und Unterstützungsangebote in Bezug auf die Förderung der frühkindlichen Entwicklung von Kindern aus anregungsarmen Familien in Berlin

Ulrich Fegeler', Elke Jäger-Roman ${ }^{2}$

'Deutsches Kinderbulletin, Öffentlichkeitsarbeit, Oranienburg, Deutschland; ${ }^{2}$ DGAAP, Pädiatrische Grundversorgung, Berlin

Die Bedeutung einer guten frühkindlichen Entwicklung für das gesamte spätere Leben ist seit langem bekannt. Kinder aus Familien des unteren
SES-Quartils haben schlechtere Entwicklungschancen und in Folge eine schlechtere Sozialprognose (Schul-, Ausbildungsabschlüsse, Arbeitslosigkeit, Delinquenz). Mit der Bundesinitiative „Frühe Hilfen“ (seit 2012) und seit 2018 der Bundesstiftung FH wurde deutschlandweit ein Netzwerk geschaffen, das durch das Vorhalten frühzeitiger, koordinierter und multiprofessioneller Angebote für werdende Eltern und in den ersten 3 Lebensjahren die Förderung der Entwicklung von Kindern zum Ziel hat. Bislang fehlen Untersuchungen dazu, wie viele Familien in belastenden Lebenslagen erreicht werden, ob die finanzielle und personelle Ausstattung der FH angemessen ist für die angenommenen $10 \%$ Familien eines Geburtsjahrganges mit psychosozialem Unterstützungsbedarf und ob sich die Entwicklung der Kinder aus diesen Familien allgemein verbessert.

Beispielhaft wird die Situation in Berlin dargestellt: 2018 kamen 43.670 Neugeborene zur Welt. 22,7 \% Kinder im Alter bis 18 Jahren leben in armutsgefährdeten und meist bildungsfernen Familien, das sind ca. 9900 Kinder des Jahrgangs 2018. Seit kurzem können alle Erstgeborenen Kinder durch den KJGD besucht werden, nicht jedoch die Nachgeborenen. $70 \%$ aller Familien werden nach der Geburt durch Hebammen betreut. Im Rahmen der FH gibt es vielfältige Angebote des Gesundheitswesens (KJGD) und der Jugendhilfe wie aufsuchende Elternhilfen, 140 Familienzentren, Ehrenamtsprojekte und z. B. 75 Familienhebammen, die z. T. nicht in Vollzeit arbeiten, sie arbeiten berlinweit derzeit 580 Wochenstunden. Aus den vielfältig erhobenen Daten zur Dokumentation der Arbeit der $\mathrm{FH}$ ist die Anzahl der unterstützten Familien nicht ableitbar, da eine $\mathrm{Fa}$ milie mehrere Angebote wahrnehmen kann (z.B. Familienhebamme plus Kurse im Familienzentrum plus Erstellung eines Sozialgutachtens durch den KJGD). Auch sind keine Zahlen bekannt, wie viele Familien Unterstützungsangebote nicht wahrnehmen wollen.

Die Berliner Schuleingangsuntersuchungen der letzten 10 Jahre lassen keine Verbesserungen im sprachlichen-, kognitiven und motorischen Bereich bei Kindern aus dem unteren SES-Quartil erkennen. $10 \%$ der Jugendlichen schaffen den Abschluss der 10. Klasse nicht. Mögliche Ursachen werden diskutiert.

\section{DGSPJ-FV 08}

FetoNeonatPfad: Integrierte Versorgung von Schwangeren und Kindern mit dem Risiko einer fetalen Wachstumsrestriktion

Lars Mense', Ekkehard Schleußner², Hans Proquitté3, Cahit Birdir', Jörg Reichert ${ }^{1}$, Jochen Schmitt ${ }^{5}$, Mario Rüdiger

'Universitätsklinikum Carl Gustav Carus Dresden, Klinik und Poliklinik für Kinder- und Jugendmedizin, Fachbereich Neonatologie \& Pädiatrische Intensivmedizin, Dresden, Deutschland;'niversitätsklinikum Jena, Klinik für Geburtsmedizin, Jena, Deutschland;'3niversitätsklinikum Jena, Klinik für Kinder- und Jugendmedizin, Jena, Deutschland; ${ }^{4}$ Universitätsklinik um Carl Gustav Carus an der Technischen Universität Dresden, Klinik und Poliklinik für Frauenheilkunde und Geburtshilfe, Dresden, Deutschland; ${ }^{5}$ Universitätsklinikum Carl Gustav Carus an der Technischen Universität Dresden, Zentrum für Evidenzbasierte Gesundheitsforschung, Dresden

Einleitung: In $5 \%$ aller Schwangerschaften ist die intrauterine Versorgung des Feten eingeschränkt und fetale Wachstumsrestriktion (FWR) eine mögliche Folge. Präeklampsie und arterielle Hypertonie sind mit FWR assoziiert und stellen ein hohes prä- und postpartales Risiko für die Mutter dar. Das Neugeborene ist durch das hohe Risiko der Frühgeburtlichkeit mit entsprechenden Morbiditäten betroffen. Unabhängig vom Geburtszeitpunkt haben FEW-Kinder ein erhöhtes Risiko späterer respiratorischer und neuro-kognitiver Probleme und entwickeln häufiger ein metabolisches Syndrom.

Sonographisch und laborchemisch können Hochrisiko-Schwangeren identifiziert werden

In den Perinatalregionen Ost-Sachsen und Ost-Thüringen wird, beginnend ab Oktober 2019, gefördert durch den Innovationsfonds und gemeinsam mit der AOK Plus Sachsen/Thüringen und der Barmer, ein integriertes Versorgungsmodell betroffener Familien von der 10. Schwangerschaftswoche bis zum Ende des 1. Lebensjahres prospektiv evaluiert. 
Methodik: Risiko-Schwangere werden anhand anamnestischer Angaben identifiziert und in den Pfad eingeschlossen. Ein Pränatalmediziner führt das kombinierte Präeklampsie-Screening (Fetal Medicine Foundation, London) durch. Ab der 20. Schwangerschaftswoche (SSW) finden risikoadaptiert vierwöchige Vorstellungen im Perinatalzentrum statt, die von interdisziplinären Fallkonferenzen, psychosozialen Interventionen und Gesprächen mit Neonatologen begleitet werden. Die Entbindung wird in einer dem Risiko angepassten Entbindungsklinik geplant und das Kind postnatal, unter besonderer Berücksichtigung der FWR, mit dem Ziel der Vermeidung von Folgeschäden betreut. Nach Entlassung werden die kinderärztlichen Vorsorgeuntersuchungen U3-U6 vertieft durchgeführt. Über den Förderzeitraum von 4 Jahren ist mit 30.500 Schwangeren in der Modellregion zu rechnen, von denen bei ca. 10.900 Schwangeren anamnestische Risiken vorliegen. Bei knapp 900 Schwangeren ist mit einem auffälligen Präeklampsie-Screening zu rechnen und ca. 500 Kinder werden im Pfad betreut, davon ca. 250 Kinder in einem Perinatalzentrum.

Evaluation: Primäres Outcome ist die geplante Entbindung in der adäquaten Entbindungsklinik und die Vermeidung von Todgeburten. Die integrierte Versorgung soll das Risiko von Frühgeburtlichkeit und die Folgen der FWR verringern, sodass eine Kostenersparnis trotz der Ausgaben für das frühe Präeklampsie-Screening und die intensivierte perinatologische Betreuung möglich ist.

\section{Poster}

\section{Entwicklung/Kinderschutz}

\section{DGSPJ-PO 01}

Münchener Funktionelle Entwicklungsdiagnostik - Überarbeitung der Urversion und Beginn der Normierungsstudie

Tamara Fuschlberger

kbo-Kinderzentrum München gemeinnützige GmbH, SPZ, Planegg,

Deutschland

Einleitung: Die Münchener Funktionelle Entwicklungsdiagnostik (MFED) wird seit vielen Jahren eingesetzt, um Entwicklungsverzögerungen bei Kindern im Alter von 0-3 Jahren aufzuzeigen. In den letzten Jahren stand das Testverfahren vermehrt in der Kritik, da die Gütekriterien bereits veraltet sind und die Zusammenstellung des Verfahrens nicht eindeutig nachvollzogen werden kann. Daher wird daran gearbeitet das Testverfahren zu überprüfen und neu zu normieren. Es wurden Abänderungen an der MFED vorgenommen, aus welchen die Neufassung entstand.

Methode: Für die Studie wurden Kinder im Alter von 0 bis 47 Monaten mit der ersten Neufassung der MFED untersucht. Soziodemografische Daten wurden anhand von einem Fragebogen erhoben.

Ergebnisse: Es nahmen 488 Kinder an den Untersuchungen teil: $49 \%$ männlich, 51 \% weiblich; die Hälfte der Eltern hatte einen Hochschulabschluss. Zur Berechnung der Reliabilität und Itemschwierigkeit wurden die Daten in drei Altersgruppen: 0-12 Monate, 13-24 Monate, 25-47 Monate, geteilt. Es zeigten sich in allen Skalen und Altersgruppen Reliabilitäten über 0,9, außer in der Skala der sozialen Entwicklung (Altersgruppe 25-47 Monate). Hier lag das Ergebnis bei 0,6. Die Ergebnisse zeigen geringe Differenzen in der Datenerhebung zwischen Medizinern und Psychologen.

Diskussion: Die Datenauswertung der Neufassung zeigt zufriedenstellende Ergebnisse. Durch die Berechnung der Itemschwierigkeit ergibt sich, dass Items umsortiert werden müssen. Schlussfolgerung: Für die Neunormierung wird ein besserer Ausgleich bezüglich der sozialen Schichten angestrebt. Um die Qualität der Datenerhebung zu sichern, werden weitere Schulungen der Untersucher durchgeführt und eine Mindestzahl von 50 Testungen pro Untersucher angestrebt.

\section{DGSPJ-PO 02}

Elterliche Belastung und die psychische Entwicklung von Kindern und Jugendlichen - Konsequenzen für die Zusammenarbeit von Pädiatrie und Kinder- und Jugendlichenpsychiatrie/psychotherapie

Viktoria Irlbauer-Müller, Oliver Kratz

Universitätsklinikum Erlangen, Kinder- und Jugendabteilung für Psychische Gesundheit, Erlangen, Deutschland

Elterliche Belastung ist als Stress zu verstehen, den Eltern bei der Erziehung ihrer Kinder erleben, wenn ihre vorhandenen Ressourcen subjektiv empfunden nicht ausreichen, um die an sie gestellten Anforderungen (kind- und elternbezogen) zu bewältigen. Dabei ist bekannt, dass elterliche Belastung die Entstehung und Aufrechterhaltung psychischer und Verhaltensauffälligkeiten seitens der Kinder fördern kann; ein Prozess, der umgekehrt eine kindbezogene Belastungserhöhung für die Eltern darstellt (Bidirektionalität/,Teufelskreis“). Die Wahrnehmung elterlicher Belastung im pädiatrischen Alltag wird so zur bedeutsamen Voraussetzung, die betroffenen Familien entsprechenden kinder- und jugendpsychiatris chen/-psychotherapeutischen (KJP) Angeboten zuzuführen. Gleichsam bedarf es der Berücksichtigung kommunikativer Besonderheiten belasteter Eltern.

Für N $=166$ zu einem KJP-Erstkontakt vorstellige Kinder und Jugendliche (11-18 Jahre) wurden KJP-relevante Merkmale (inkl. kritischer Lebensereignisse), die elterliche Belastung und drei Messungen der kindseitigen 
Symptomatik (1. Elternurteil, 2. Selbsturteil, 3. klinisches Urteil) unter epidemiologischen Fragestellungen betrachtet. Zudem sollte die Hypothese geprüft werden, ob belastete Eltern valide Auskünfte über die psychische und verhaltensbezogene Symptomatik ihrer Kinder geben können.

Die befragten Eltern zeigten sich auffällig hoch belastet. Diese Belastung korrelierte hoch bedeutsam mit kritischen Lebensereignissen sowie mit dem Elternurteil. Verglichen mit dem Selbst- und dem klinischen Urteil erwies sich nur das Elternurteil als hoch signifikanter Prädiktor elterlicher Belastung, die für verschiedene KJP-Merkmale variierte. Die Inanspruchnahme der KJP-Behandlung wurde seitens der Eltern zumeist als Entlastung erlebt. Für auffällig belastete Eltern fanden sich in Mittelwertvergleichen größere Differenzen aus Eltern- und klinischem Urteil als für unauffällig belastete Eltern. Korrelationen zeigten zudem, dass eine höhere elterliche Belastung mit größeren Urteilsdifferenzen einherging. Die multiple Regression belegte dabei die Vorhersagekraft kindbezogener elterlicher Belastung für die Urteilsdifferenz.

Schlussfolgernd sollte die Belastung von Eltern bereits unter präventiven Gesichtspunkten, wie es im Bereich des pädiatrischen Vorsorgesystems geschehen kann, systematisch erfasst werden, um betroffenen Familien möglichst frühzeitig entsprechende Hilfsangebote zu unterbreiten. Zudem sollten Elternangaben bezüglich der psychischen und Verhaltensauffälligkeiten ihrer Kinder im Falle elterlicher Belastung kritisch reflektiert werden, um deren Einbezug in diagnostische und therapeutische Entscheidungen sorgfältig abzuwägen.

\section{DGSPJ-PO 03 \\ „Unser kleiner Schreihals": Smartphone-gestützte Psychoedukation für Eltern von Kindern mit Regulationsproblemen}

\section{Michaela Augustin, Maria Licata-Dandel, Volker Mall, Anna Friedmann}

Technische Universität München, Lehrstuhl für Sozialpädiatrie, München, Deutschland

Hintergrund: Frühkindliche Regulationsprobleme wie häufiges Schreien, Schwierigkeiten beim Ein- oder Durchschlafen und/oder beim Füttern stellen für Eltern und Kind im Alltag häufig eine enorme Belastung dar. Betroffene Eltern erleben sich zudem oft als wenig selbstwirksam in ihrer Elternrolle und als sozial isoliert. Die neu entwickelte App „Unser kleiner Schreihals" zielt auf Psychoedukation im Bereich frühkindlicher Regulationsprobleme ab und soll als niederschwelliges frühzeitiges Unterstützungsangebot dienen. Zusätzlich zu Informationstexten und Expertenvideos enthält die App eine Tagebuch-Funktion zur SymptomDokumentation, ein Eltern-Austauschforum sowie ein bayernweites Anlaufstellenverzeichnis.

Fragestellung: Übergeordnetes Ziel der Studie ist die Wirksamkeitsevaluation der App „Unser kleiner Schreihals“. Es wird untersucht, ob die AppNutzung zu einer Reduktion der elterlichen Belastung führt (1), den elterlichen Wissensstand über Regulationsprobleme (2) und das elterliche Selbstwirksamkeitsempfinden (3) erhöht, eine Reduktion der kindlichen Symptombelastung bewirkt (4) sowie die elterliche erlebte soziale Unterstützung verbessert (5).

Material und Methoden: Die Wirksamkeit der App wird anhand einer monozentrischen, prospektiven, randomisiert-kontrollierten Interventionsstudie (RCT) mit Wartekontrollgruppendesign überprüft, gefördert durch die Initiative „Gesund.Leben.Bayern“ des Bayerischen Staatsministeriums für Gesundheit und Pflege. Hierfür werden insgesamt $N=136$ Elternteile von Kindern im Alter von 0-24 Monaten, die sich in der Schreiambulanz des kbo-Kinderzentrums München zu einem Erstberatungstermin anmelden, rekrutiert. Während der Interventionsgruppe für die Dauer der regulären Wartezeit bis zum Erstberatungstermin in der Schreiambulanz (4-6 Wochen) die App zur Verfügung gestellt wird, erhält die Wartekontrollgruppe diese erst nach dem Beratungstermin (=Studienende). Beide Gruppen werden hinsichtlich der elterlicher Belastung, des Wissenstandes über Regulationsprobleme, des elterlichen Selbstwirksamkeitsempfindens, der kindlichen Symptomausprägung sowie der erlebten elterlichen sozialen Unterstützung zu zwei Messzeitpunkten (prä-post) anhand von
Fragebögen untersucht. Ein Evaluationsfragebogen erfasst zudem das Nutzerverhalten der Probanden sowie die Anwenderfreundlichkeit und Konzeption der App.

Ergebnisse: Es erfolgt eine Präsentation der Zwischenergebnisse der aktuell laufenden Studie.

Schlussfolgerung/Diskussion: Bei positiven Ergebnissen soll das niederschwellige Angebot zukünftig kostenfrei zur Verfügung gestellt werden. Die App soll eine Ergänzung zur etablierten professionellen Beratung für Eltern von Kindern mit Regulationsproblemen darstellen, indem sie über die Bereiche Schreien, Schlafen und Füttern angemessen informiert. Durch eine gezielte Aufklärung über Behandlungsmöglichkeiten können betroffene Familien zur frühzeitigen Annahme professioneller Unterstützung motiviert werden.

\section{DGSPJ-PO 04 \\ Belastungen und Ressourcen in der therapeutischen Arbeit mit Kindern und Jugendlichen}

\section{Sarah Bien, Oliver Kratz, Viktoria Irlbauer-Müller}

Universitätsklinikum Erlangen, Kinder- und Jugendabteilung für Psychische Gesundheit, Erlangen, Deutschland

Ziel nachfolgender Untersuchung war es, sich mit berufsbezogenen Belastungen und Ressourcen von Therapeuten in der Kinder- und Jugendpsychiatrie und -psychotherapie, sowie mit deren Zusammenhang zu Wohlbefindens- und Belastungsmaßen auseinanderzusetzen. Hierzu wurde ein Fragebogen erstellt, der neben diverser demographischer Daten Belastungen und Ressourcen von therapeutisch tätigen Personen, Wohlbefinden, emotionale Erschöpfung, Erfolgsdruck sowie die selbsteingeschätzte Therapieeffektivität erfragte. Dieser Fragebogen wurde im Paper-Pencil-Verfahren von $\mathrm{N}=66$ Therapeuten aus 3 verschiedenen Kliniken für Kinderund Jugendpsychiatrie und -psychotherapie ausgefüllt. In explorativen Analysen der bisher kaum untersuchten Belastungs- und Ressourcenskalen zeigte sich vor allem die große Bedeutung sozialer Belastungen und Ressourcen für unerfahrene sowie in Ausbildung befindliche Therapeuten. Negative Zusammenhänge zwischen dem Wohlbefinden und der emotionalen Erschöpfung sowie zwischen dem Wohlbefinden und den wahrgenommenen Belastungen sprechen für die Notwendigkeit, sich zukünftig in der Forschung mit berufsbezogenen Belastungen und Ressourcen von Therapeuten im kinder- und jugendpsychiatrischen/-psychotherapeutischen Kontext auseinanderzusetzen, um im Sinne der Qualitätssicherung den Patienten eine optimale Behandlung bieten zu können. Gelingt es Kliniken durch Reduktion der Belastungsfaktoren und ausreichende Bereitstellung von Ressourcen die Gesundheit und das Wohlbefinden ihrer angestellten Therapeuten aufrechtzuerhalten, können bessere Behandlungserfolge erzielt werden. Eine Prüfung der Generalisierbarkeit für den pädiatrischen Bereich ist ausstehend, wenngleich auf Basis des gegenwärtigen Erkenntnisstandes von einer gewissen Vergleichbarkeit ausgegangen werden kann.

\section{DGSPJ-PO 05}

Umsetzung diagnostischer Standards bei Sprachauffälligkeiten: Eine Kasuistik im Längsschnitt

\section{Falko Dittmann, Anke Buschmann}

Zentrum für Entwicklung und Lernen, Diagnostik und Beratung, Heidelberg, Deutschland

Hintergrund: Die S2k-Leitlinie für SES sieht für die Sprachdiagnostik einen mehrstufigen, interdisziplinären Prozess vor. Ziele sind die differenzialdiagnostische Abklärung, die Ermittlung möglicher Störungsschwerpunkte und damit das Entscheiden über Therapieindikationen. Dieses Vorgehen findet in der klinischen Praxis jedoch nicht konsequent Anwendung. Insbesondere bei mehrsprachig aufwachsenden Kindern wird eine langsam voranschreitende Sprachentwicklung häufig fälschlicherweise der Mehrsprachigkeit zugeschrieben und infolgedessen keine frühzeitige differenzialdiagnostische Abklärung initiiert. Diese ist jedoch wichtig, da 
eine deutlich verzögerte Sprachentwicklung mit 24 Monaten ein Indiz für schwerwiegende Auffälligkeiten sein kann, wie eine globale Entwicklungsstörung oder eine Autismus-Spektrum-Störung (Buschmann et al., 2008). Zielsetzung: Im Rahmen einer Kasuistik wird die Umsetzung des diagnostischen Prozesses und dessen Standards gemäß der S2k-Leitlinie im Längsschnitt vorgestellt.

Probanden und Methoden: Es handelt sich um ein simultan zweisprachig (georgisch/deutsch) aufwachsendes Mädchen, welches zur U7 über einen deutlich eingeschränkten aktiven Wortschatz in beiden Sprachen verfügte ( $<10$ Wörter). Bei der Erstvorstellung mit 2;0 Jahren erfolgte eine Beurteilung der Allgemeinentwicklung (Bayley-III-Skalen) sowie der sprachlichen Fähigkeiten (expressiv und rezeptiv) mittels SETK-2. Verlaufsuntersuchungen fanden mit 3;2, 3;7 und 4;3 Jahren statt. Hierbei erfolgte je eine standardisierte Abklärung der nonverbalen kognitiven Fähigkeiten (SON-R 21/2-7) und der rezeptiven-expressiven Sprachkompetenzen auf Deutsch (SETK-3-5; TROG-D). Zudem wurde informell das Sprachverständnis im Georgischen erfasst. Eine Verhaltensbeobachtung und eine Spontansprachanalyse ergänzten die Testverfahren.

Ergebnisse: Mit 2;0 Jahren verfügte das Mädchen über eine altersentsprechende Allgemeinentwicklung. Ihre rezeptiven und expressiven Sprachfähigkeiten waren in beiden Sprachen deutlich beeinträchtigt. Somit wurde eine isoliert rezeptiv-expressive Sprachentwicklungsverzögerung (F.89.0; Late Talker) diagnostiziert. Der Sprachrückstand beider Sprachen wurde trotz häuslicher Sprachanregung, Kita-Besuchs und Logopädie nicht aufgeholt: Mit drei Jahren und auch später wies sie eine Umschriebenen Entwicklungsstörung des Sprechens und der Sprache (F.80.2; rezeptiv-expressive SES) auf, von der beide Sprachen betroffen waren. Die nonverbalen kognitiven Fähigkeiten waren zu allen Untersuchungszeitpunkten altersentsprechend.

Schlussfolgerung: Die weiterhin bestehenden sprachlichen Schwierigkeiten verunsichern und belasten die Eltern zunehmend. Sie sorgen sich um die weitere Entwicklung, insbesondere hinsichtlich einer Regelbeschulung. Eine Beratung im Hinblick auf Fragen zur mehrsprachigen Erziehung sowie weitere Unterstützungsmöglichkeiten ist indiziert. Der Entwicklungsverlauf sollte weiterhin überprüft und die therapeutischen Schwerpunkte ggf. angepasst werden.

\section{DGSPJ-PO 06}

Interdisziplinäre Zusammenarbeit der Hochschule für Gesundheit Bochum und der Kinderklinik des St. Josef-Hospitals Bochum (Universitätsklinik RUB): Logopädische Diagnostik in der interdisziplinären Zusammenarbeit mit der Neuropädiatrie

\section{Claudia Herhold, Sylvia Costard}

Hochschule für Gesundheit Bochum, Department für angewandte Gesundheitswissenschaften, Studienbereich Logopädie, Bochum, Deutschland

Seit Herbst 2013 kooperiert der Studienbereich Logopädie des Departments für Angewandte Gesundheitswissenschaften der Hochschule für Gesundheit (hsg) mit der neuropädiatrischen Abteilung des St. Josef-Hospitals in Bochum (Universitätsklinikum Ruhr Universität Bochum). Initiiert wurde das Projekt von Prof. Dr. Sylvia Costard, Leitung des Studienbereichs Logopädie, und Prof. Dr. Thomas Lücke, Direktor der Klinik für Kinder- und Jugendmedizin und Leiter der Abteilung Neuropädiatrie mit Sozialpädiatrie.

Primäres Ziel der interprofessionellen Kooperation des Studienbereichs Logopädie und der Abteilung Neuropädiatrie ist es insbesondere, herauszufinden, ob ein Zusammenhang von Sprach- und Schriftsprachstörungen und Rolando-Epilepsien (idiopathisch fokalen Epilepsien) generiert werden kann. Die Prävalenz von Epilepsien im Kindesalter beträgt 0,5 \% (Neubauer, Groß \& Hahn 2008). Insgesamt macht der Anteil von Kindern etwa $25 \%$ aller Neuerkrankungen aus. (ebd.) Laut Finetti (2009) weisen ca. 6-8 \% aller Kinder mit einer Epilepsie Sprachstörungen auf.

Bei der Auswertung der Ergebnisse von untersuchten Kindern mit einer idiopathisch fokalen Epilepsie kooperieren wir mit dem ansässigen Psychologen im Universitätsklinikum, da etwa 70 \% aller betroffenen Kinder eine Komorbidität in Bereich der Intelligenzminderung aufweisen. Die logopädische Diagnostik soll sich jedoch auf die sprachlichen Leistungen in allen sprachsystematischen Ebenen konzentrieren. Dazu werden innerhalb der logopädischen Diagnostik standardisierte Testverfahren eigesetzt, die speziell den Wortschatz untersuchen. Orientierend werden aber auch die pragmatisch-kommunikative, phonetisch-phonologische sowie morphologisch-syntaktische Ebene beobachtet. Durch die enge Verzahnung von Praxis, Interdisziplinarität und Lehre im Rahmen der wöchentlichen Patientenvorstellungen werden die Studierenden praxisnah an ihr Berufsleben als Logopäden/Innen herangeführt. Und innerhalb der Forschungsfrage können wir Daten sammeln, um eventuell Zusammenhänge generieren zu können.

Durch die enge Verzahnung von Praxis und Lehre im Rahmen der wöchentlichen Patientenvorstellungen werden die Studierenden praxisnah an ihr Berufsleben als Logopäden/Innen herangeführt. Aber auch die Abteilung Neuropädiatrie profitiert von der Kooperation, denn die Studierenden arbeiten sich in Gebiete ein, in denen noch ein großer Forschungsbedarf besteht, so z. B. in den oben genannten Zusammenhang von Sprach- und Schriftsprachstörungen und Epilepsien. Die enge Kooperation ermöglicht Studierenden Projekt- und Bachelorarbeiten zu klinischen Fragestellungen zu schreiben, die zu weiteren Erkenntnissen im neuropädiatrischen Bereich führen. Aus der Kooperation resultiert also zum einen eine Erweiterung des Lehrangebotes an der hsg mit engem Bezug zum späteren Berufsbild. Zum anderen können auch Erkenntnisse zu neuropädiatrischen Sprachstörungen gewonnen und die Forschung vorangetrieben werden.

\section{Literatur}

1. Boscariol M (2015) Language and central temporal auditory processing in childhood epilepsies. Epilepsy Behav 53(2015):180-183

2. Lücke T, Costard S, Illsinger S (Hrsg) (2017) Neuropädiatrie für Sprachtherapeuten. Elsevier, München

\section{DGSPJ-PO 07}

Schwerer Verlauf bei initial neurologisch unauffälligem Säugling nach „Schütteltrauma“ (Nicht akzidentellem Schädelhirn-Trauma)

Stephanie Lehmann-Kannt, Marina Akçay, Michael Zemlin

Universitätsklinikum d. Saarlandes, Klinik für Allgemeine Pädiatrie und Neonatologie, Homburg/Saar, Deutschland

Hintergrund: Intrakranielle Verletzungen können sich von der Symptomfreiheit bis zu komatösen Zuständen und Apnoen präsentieren. Die Haut ist bei Kindern mit nicht-akzidentellen Verletzungen das Organ, welches am häufigsten betroffen ist.

Fallbericht: Wir berichten von einem 3 Monate alten Säugling, der mit multiplen Hämatomen am Thorax, Knie und Kinn sowie radiologisch älteren und frischen Rippenfrakturen in unsere Klinik zur Überwachung und weiteren Diagnostik verlegt wurde. Die Eltern berichteten, dass die Ursache der Verletzungen ein Sturz der 16 Monate alten Tochter vom Sofa auf ihren Bruder am Vorstellungstag gewesen sei. Dieser habe vor dem Sofa auf dem Bauch gelegen und geschlafen.

Im Aufnahmeuntersuchungsbefund war der Säugling neurologisch unauffällig. In der augenärztlichen Untersuchung fanden sich frische, retinale Blutungen und im kraniellen MRT zeigten sich beidseitige Subduralhämatome unterschiedlichen Alters sowie ausgeprägte Ischämien in sämtlichen Stromgebieten beider Hemisphären links mehr als rechts. Im Ganzkörperröntgen fielen neben einer älteren, linksseitigen Klavikulafraktur multiple, ältere Rippenfrakturen mit Kallusbildung sowie mehrere frische Rippenfrakturen und metaphysäre Kantenabsprengungen an beiden distalen Tibiametaphysen auf.

Auf Grund des ausgeprägten MRT-Befundes erfolgte vorsorglich die Verlegung auf die Intensivstation. Einige Stunden nach Aufnahme begannen komplex-fokale Krampfanfälle bis zum Status epilepticus, der medikamentös nicht durchbrochen werden konnte. Daher wurde der Patient intubiert und beatmet und mit Thiopental behandelt. Hierunter besserten sich die pathologischen EEG-Befunde sukzessive, so dass der Patient nach 4 Tagen 
extubiert werden konnte. Die medikamentöse Behandlung wurde mit zwei Antiepileptika fortgesetzt.

Die Summe und die Art der Verletzungen ist nicht mit dem von den Eltern geschilderten Unfallmechanismus vereinbar. Es handelt sich ohne Zweifel um ein NASHT (Nicht akzidentellem Schädelhirn-Trauma) - früher als „Schütteltrauma“ bezeichnet.

Schlussfolgerung: Bei jedem Verdacht auf Kindsmisshandlung sollte auch bei scheinbar nicht lebensbedrohlichem Verletzungsmuster - zeitnah nach eventuell noch asymptomatischen, schweren, inneren (Begleit-) Verletzungen wie z. B. Nicht akzidentelles Schädelhirn-Trauma oder Verletzungen der Abdominalorgane gesucht werden. Jedes Kind mit Verdacht auf Kindesmisshandlung kann sich als akuter Notfall entpuppen.

\section{DGSPJ-PO 08}

\section{Intrakranielle Hygrome im Säuglingsalter - mehr als ein Hinweis auf Schütteltrauma}

Felix Timpe ${ }^{1}$, Anne Jaabusch', Thomas Kunz ${ }^{3}$, Thorsten Wygold'

${ }^{1}$ Westküstenkliniken Heide, Klinik für Kinder- und Jugendmedizin, Heide, Deutschland; ${ }^{2}$ Westküstenklinikum Heide, Institut für diagnostische und interventionelle Radiologie/Neuroradiologie, Heide, Deutschland; ${ }^{3}$ Westküstenklinikum Heide, Frauenklinik, Heide, Deutschland

Einleitung: Isolierte intrakranielle Hygrome ohne hämostaseologische Grunderkrankung im Säuglingsalter gelten als wegweisend für 2 Diagnosen: Schütteltrauma und Glutarazidurie Typ 1. Die folgende Kasuistik soll auf eine 3. Ursache hinweisen, die offensichtlich häufiger ist als die Möglichkeit der Stoffwechselerkrankung.

Fallvorstellung: Ein zum Zeitpunkt der Vorstellung drei Monate alter Patient wurde bei Wohlbefinden aufgrund einer angeblich akut aufgetretenen $3 \times 5 \mathrm{~cm}$ großen, fluktuierenden Schwellung am Hinterkopf vorgestellt. Das Kind war bislang internistisch und neurologisch stets unauffällig gewesen. Bei sonographischem Verdacht auf Enzephalozele wurde eine Bildgebung des Schädels im 3T-MRT durchgeführt, in der intrakraniell frontal gelegene Hygrome und punktförmige Einblutungen auf den Kleinhirntonsillen auffielen. Die Glutarazidurie Typ 1 und eine Gerinnungsstörung konnten ausgeschlossen werden. Insofern komplettierten wir unter dem Verdacht auf ein Schütteltrauma die im Behandlungspfad Kinderschutz hinterlegte Diagnostik und informierten, wie im Pfad festgelegt, Jugendamt und Rechtsmedizin. Die spezifische Abklärung ergab aber keine weiteren Hinweise auf zugefügte Gewalt. Gleichzeitig beteuerten die Eltern eindrücklich, das Kind nie geschüttelt zu haben, so dass wir den Fall weiter aufarbeiteten. Dass das Kind per Vakuumextraktion entbunden worden war, war bereits vorher bekannt gewesen. Nach Angaben der beteiligten Geburtshelfer und auch so dokumentiert war die VE nur kurz und ohne Probleme verlaufen. Das Kind war postpartal unauffällig geblieben. Schlussfolgerung: Unsere Literaturrecherche ergab, dass auch bei komplikationsloser und erfolgreicher Vakuumextraktion eine Inzidenz subduraler Hämatome von 1:860 VE besteht (1). Insofern ist im vorliegenden Fall bei fehlenden Alternativen die Ausbildung der Hygrome auf diesem Wege denkbar, auch wenn die überwiegende gynäkologische Standardliteratur in Deutschland diese Komplikation nach lege artis durchgeführter Vakuumextraktion als eigentlich nicht möglich beschreibt (2).

52 von 1000 Entbindungen werden in Deutschland per Vakuumextraktion durchgeführt (2). Daraus errechnet sich eine Inzidenz subduraler Hämatome nach VE von 1:16.500 Geburten, die damit höher ist als die Inzidenz der Glutarazidurie Typ 1 (1:156.000 Geburten) (3). Bei zufällig entdeckten Hygromen des Säuglingsalters muss daher auch eine Entbindung mit unauffällig verlaufener Vakuumextraktion als mögliche Ursache angemessen berücksichtigt werden.

\footnotetext{
Literatur

1. Ali UA, Norwitz ER (2009) Vacuum-Assisted Vaginal Delivery. Rev Obstet Gynecol 2(1):5-17

2. Deutsche Gesellschaft für Gynäkologie und Geburtshilfe. Vaginal-operative Entbindungen. S1-Leitlinie, AWMF-Register-Nr. 015-023, in der Fassung vom
}

31.05.2012, www.awmf.org/leitlinien/detail/ll/015-023.html, download am 13. Mai 2019

3. Deutsche Gesellschaft für Kinderheilkunde und Jugendmedizin e. V. Glutarazidurie Typ I, Diagnostik, Therapie und Management. S3-Leitlinie, AWMF-RegisterNr. 027-018, in der Fassung vom 01.06.2016, www.awmf.org/leitlinien/detail/ Il/027-018.html, download am 13. Mai 2019.

\section{DGSPJ-PO 09}

\section{Schulabsentismus: Ein Hinweis auf eine Kindeswohlgefährdung?}

Christina Beisenherz, Angelika Konrad, Anna Meyer-Borgstädt, Anna Franz Landeshauptstadt München, Referat für Gesundheit und Umwelt, Gesundheitsvorsorge für Kinder- und Jugendliche, München, Deutschland

In der Schulärztlichen Sprechstunde der Landeshauptstadt München nimmt die Zahl der Vorstellungen von Kindern und Jugendlichen aufgrund hoher Fehlzeiten in der Schule in den letzten Jahren zu. Die Genese des Schulabsentismus ist multifaktoriell, psychosoziale Belastungsfaktoren liegen in der Regel vor. Vernachlässigung, aber auch andere Formen der Kindeswohlgefährdung, wie körperliche Misshandlung, häusliche Gewalt und sexueller Missbrauch werden im Zusammenhang mit Schulabsentismus beschrieben. Zudem kann Schulabsentismus bereits für sich mit weitreichenden negativen Folgen für die Gesundheit und Entwicklung einhergehen. Die mangelnde soziale Teilhabe, fehlende altersentsprechende Förderung und das drohende schulische Versagen stellen erhebliche Risiken dar. Hinweise für das Vorliegen einer Kindeswohlgefährdung sollten damit im Rahmen der interdisziplinären Abklärung von Schulabsentismus geprüft werden.

Im Schuljahr 2017/18 wurden 200 minderjährige Schülerinnen und Schüler aufgrund von Schulabsentismus in der Schulärztlichen Schulsprechstunde kinder- und jugendärztlich begutachtet. In 47 Fällen erfolgte die Kontaktaufnahme einschließlich einer schriftlichen Stellungnahme zu dringend erforderlichen ärztlich-therapeutischen Maßnahmen an das zuständige Jugendamt. Der Grund war in über $90 \%$ das Vorliegen von Schulabsentismus und Hinweisen auf Vernachlässigung bei mangelnder elterlicher Mitwirkung in der Umsetzung der indizierten medizinischen Maßnahmen. Die Bedeutung der intensiven Zusammenarbeit aller beteiligten Berufsgruppen und Akteure in der weiteren Abklärung von Schulabsentismus mit Hinweisen auf Kindeswohlgefährdung wird anhand eines Fallbeispiels dargestellt. 


\section{Posterbereich DGSPJ - Impfungen/Neonatologie/ Arzneimittel}

\section{DGSPJ-PO 10}

\section{Inanspruchnahme von Standardimpfungen für Kinder und Jugendliche - Entwicklungen seit der KiGGS-Basiserhebung}

Christina Poethko-Müller', Ronny Kuhnert', Sofie Gillesberg Lassen², Anette Siedler $^{2}$

'Robert Koch-Institut, Abteilung für Epidemiologie und

Gesundheitsberichterstattung, Berlin, Deutschland;'Robert Koch-Institut, Abteilung für Infektionsepidemiologie, Berlin, Deutschland

Die Rahmenbedingungen des Impfens haben sich in Deutschland seit der Basiserhebung der "Studie zur Gesundheit von Kindern und Jugendlichen in Deutschland (KiGGS)“ 2003-2006 verändert und zusätzliche Impfungen sind in den Impfkalender aufgenommen worden. Die aktuellen Daten der KiGGS Studie (KiGGS Welle 2) erlauben für die Geburtsjahrgänge von 1985 bis 2013 eine Beurteilung des Impfstatus und der zeitlichen Veränderung.

Von insgesamt 15.023 Teilnehmenden an KiGGS Welle 2 (Response 40,1\%) nahmen 3238 drei- bis 17-Jährige am Untersuchungsprogramm teil, legten ihren Impfausweis vor oder waren laut Elternangabe ungeimpft. Im Vergleich zu Gleichaltrigen vor 10 Jahren sind die Impfquoten angestiegen. Das gilt insbesondere für Impfungen, für die in KiGGS-Basis noch starke Defizite bestanden, wie z. B. die Hepatitis B- und 2. Masernimpfung in allen Altersgruppen, die Pertussisimpfung- (11- bis 17-Jährige) sowie die Auffrischimpfung gegen Tetanus bei den 7- bis 10-Jährigen.

Trotz deutlicher Anstiege liegen jedoch auch bei den jüngsten Geburtskohorten die Impfquoten zum Ende des 2. Lebensjahres für alle Impfungen noch weit unter 95 \% (z. B. 1. Masern: 88,6 \%; 2. Masern: 64,4 \%).

Weiterhin zeigen sich Unterschiede beim Impfstatus in Abhängigkeit von soziodemografischen Faktoren und weniger als jedes zweite Kind ist gegen Hepatitis B geimpft (45,9\%), wenn Eltern Angst vor Nebenwirkungen oder eine impfskeptische Haltung als Gründe gegen Impfungen nennen. Nach Elternangaben wurden 3,2 \% der Kinder ärztlich von einer oder mehreren Impfungen abgeraten, am häufigsten wurde von Impfungen gegen Varizellen, FSME, Rotaviren, Meningokokken, HPV und Hepatitis B abgeraten. Die Gründe dafür unterscheiden sich zwischen den Impfungen. Die Ergebnisse zeigen, wo noch weitere Bemühungen notwendig sind, um die Impfquoten um die verbleibenden letzten Prozentpunkte zu steigern und die zeitgerechte Gabe aller im Impfkalender aufgeführten Impfungen sowie die gesetzten Eliminationsziele zu erreichen.

\section{DGSPJ-PO 11}

\section{Comparison of vaccination strategies against respiratory syncytial virus: impact on disease burden and costs/VIPER}

Marina Treskova', Stefan Flasche ${ }^{2}$, Viktoria Schönfeld ${ }^{3}$, Francisco Pozo Martin $^{3}$, Stefan Scholz ${ }^{3}$, Kathrin Thöne ${ }^{4}$, Udo Schneider', Thomas Harder ${ }^{3}$, Mark Jit ${ }^{2}$, Ole Wichmann ${ }^{3}$

'Robert Koch-Institut Berlin, Abteilung für Infektionsepidemiologie, Berlin, Germany; ${ }^{2}$ London School of Hygiene and Tropical Medicine, Department of Infectious Disease Epidemiology, London, United Kingdom; ${ }^{3}$ Robert KochInstitut, Abteilung für Infektionsepidemiologie, Berlin, Germany; ${ }^{4}$ Techniker Krankenkasse, Versorgungsmanagement - Entwicklung, Hamburg, Germany

Background: Respiratory syncytial virus (RSV) is one of the leading causes of lower respiratory tract infections in children which may lead to complications and death. Premature infants, children with cardiac or pulmonary conditions and immunosuppressed persons are at high risk for complications. For these individuals, palivizumab (monoclonal antibody) is recommended by the Association of the Scientific Medical Societies in Germany (AWMF) for prevention of RSV infections. There is no approved vaccine against RSV, however, a vaccine candidate for pregnant women is currently undergoing a phase III clinical trial.
Due to the absence of RSV-specific surveillance systems in Germany, there are no available estimates of RSV incidence and hospitalizations. Also the economic burden of RSV infections in Germany has not been estimated. Objective and research questions: We aim to investigate the incidence of RSV infections and hospitalizations and estimate usage of healthcare resources attributed to RSV.

The main goal is to synthesise evidence for the German Standing Committee on Vaccination (STIKO) about the most effective and cost-effective RSV-vaccination strategy. The questions such as „how many vaccinations are needed to avoid a RSV-related hospitalization?" and „how many Euros are to be spent to generate an additional quality-adjusted life year (QALY)?" will be addressed and answered.

Data and methods: Estimating RSV incidence and usage of healthcare resources will be performed using claims data of Techniker Krankenkasse (TK) for the period 2015-2018. Incidence will be estimated using ICD-10GM code. RSV-attributable usage of resources will be assessed based on incremental differences between RSV cases and controls using multivariate analyses. The control sample will be identified using matching methods. To project RSV infections and hospitalizations under no vaccination, immunization with palivizumab and different vaccination scenarios a compartmental age-structured model for RSV transmission will be developed. The model will be informed with the demographic structure and a contact matrix of the German population. The model will be parameterised using the RSV incidence data.

The economic efficiency will be estimated as costs per life year- and QALY gained and costs per avoided hospitalization.

Expected results: Results are expected in 2021. These include the estimated disease burden in terms of age-specific incidence of RSV infections, number and duration of hospitalizations, usage of other healthcare services and loss of productivity. Economic evaluations will outline an optimal strategy, probable cost for implementation and its potential in reducing RSV infections and hospitalizations.

Conclusion: These outcomes are expected to inform recommendations of STIKO about the RSV-vaccination strategies and improve healthcare provision for the German population.

Funding: The Innovation Fund of the Joint Federal Committee (Grant number 01VSF18015)

\section{DGSPJ-PO 12}

\section{Auffrischimpfung gegen Meningokokken der Serogruppe C: Abschätzung der Effekte auf Krankheitslast und Kosten im deutschen Gesundheitssystem/AMSeC}

Viktoria Schönfeld ', Francisco Pozo Martin², Stefan Scholz², Marina Treskova², Ole Wichmann ${ }^{2}$, Thomas Harder ${ }^{2}$

${ }^{1}$ Robert Koch-Institut, Fachgebiet Impfprävention, Berlin, Deutschland;'Robert Koch-Institut, Abteilung für Infektionsepidemiologie, Berlin, Deutschland

Hintergrund: Invasive Meningokokken-Erkrankungen sind selten, aber manifestieren sich häufig in Form einer Meningitis oder Sepsis. Sie werden durch Neisseria meningitidis ausgelöst und betreffen insbesondere das Kindes- und Jugendalter. Komplikationen, die hohe Letalität und potentielle Spätschäden haben große gesundheitsökonomische Auswirkungen. Trotz der seit 2006 von der Ständigen Impfkommission (STIKO) empfohlenen Standardimpfung aller Kinder im Alter von 12 bis 23 Monaten gegen Meningokokken der Gruppe C (MenC) reicht der Herdenschutz zur Verhinderung aller Erkrankungsfälle nicht aus. Die Wirksamkeit der MenCImpfung lässt über die Zeit nach, sodass eine mögliche Auffrischimpfung im Jugendalter diskutiert wird.

Fragestellungen: Ist eine Auffrischimpfung gegen MenC im Jugendalter epidemiologisch sinnvoll und kosteneffektiv?

Welche Impfstrategie kann die meisten Fälle verhindern und ist am kosteneffektivsten?

Studienziele: Primäres Ziel ist es, die Effekte einer möglichen Auffrischimpfung gegen MenC bzw. gegen Meningokokken der Serogruppen ACWY (MenACWY) im Jugendalter auf die konkrete Versorgungssitu- 
ation in Deutschland unter Berücksichtigung der Krankheitslastreduktion und der ökonomischen Folgen abzuschätzen. Sekundäres Ziel ist die Pilotierung der 2016 von der STIKO implementierten „Methoden zur Durchführung und Berücksichtigung von Modellierungen zur Vorhersage epidemiologischer und gesundheitsökonomischer Effekte von Impfungen für die Ständige Impfkommission“. Nachfolgend wird das Studiendesign vorgestellt.

Material und Methoden: Mit Hilfe eines dynamischen Transmissionsmodells (altersstrukturiertes Kompartmentmodell) für die deutsche Bevölkerung soll eine Prognose über die zukünftige Inzidenz von MenC- bzw. MenACWY-Erkrankungen sowie über die zusätzlichen Kosten pro gewonnenem qualitätsadjustierten Lebensjahr (QALY) unter verschiedenen Impfstrategien simuliert werden. Meldedaten und eine Kontaktmatrix für die deutsche Bevölkerung werden in das Modell einfließen. Zur Schätzung der Impfeffektivität und der Dauer des Impfschutzes werden systematische Reviews durchgeführt. QALYs werden in einer Substudie bei je 265 Fällen und Kontrollen mittels standardisierter und validierter Fragebögen zur gesundheitsbezogenen Lebensqualität (EQ 5D/HUI3/PedsQL) erhoben. Zur Schätzung der durch die Meningokokken-Erkrankungen verursachten Kosten werden Auswertungen von Krankenkassen-Abrechnungsdaten, Interviews mit klinischen Experten, Literatur sowie eine Befragung von Erkrankten zu direkten und indirekten Kosten im Rahmen ihrer Erkrankung herangezogen.

Erwartete Ergebnisse: Ergebnisse sind Anfang 2022 zu erwarten und sollen die STIKO bei ihrer Entscheidungsfindung zu der Fragestellung unterstützen, ob zusätzlich zur Standard-MenC-Impfung von Kleinkindern eine Impfung im Jugendalter gegen MenC oder MenACWY empfohlen werden sollte.

Gefördert durch den Innovationsfond beim Gemeinsamen Bundesausschuss (Förderkennzeichen 01VSF18017).

\section{Literatur}

1. STIKO (2016), Methoden zur Durchführung und Berücksichtigung von Modellierungen zur Vorhersage epidemiologischLiteraturer und gesundheits-ökonomischer Effekte von Impfungen für die Ständige Impfkommission, Version 1.0 (Stand: 16. März 2016), Berlin

\section{DGSPJ-PO 13}

\section{INSIST: Impact des Neonatologie-Screenings auf Infektionsprävention/Senkung von Transmission}

Tanja Artelt', Martin Kaase', Helmut Küster' ${ }^{2}$, Martina Lange' ${ }^{2}$, Thomas Paul', Steffen Unkel', Helmut Eiffert', Diana Fenz' ${ }^{1}$, Sonja Poklekowski', Tim Friede ${ }^{3}$, Simone Scheithauer?

'Universitätsmedizin Göttingen, Georg-August-Universität Göttingen, Institut für Krankenhaushygiene und Infektiologie, Göttingen, Deutschland; 2Universitätsmedizin Göttingen, Georg-August-Universität Göttingen, Klinik für Pädiatrische Kardiologie und Intensivmedizin, Göttingen, Deutschland; ${ }^{3}$ Universitätsmedizin Göttingen, Georg-August-Universität Göttingen, Institut für Medizinische Statistik, Göttingen, Deutschland; ${ }^{4}$ Universitätsmedizin Göttingen, Georg-August-Universität Göttingen, Institut für Medizinische Mikrobiologie, Göttingen, Deutschland

Fragestellung: Seit dem Jahr 2012 empfiehlt die Kommission für Krankenhaushygiene und Infektionsprävention am Robert Koch-Institut (KRINKO) ein generelles mikrobielles Kolonisationsscreening bei Früh- und Neugeborenen auf neonatologischen Intensivstationen. Die Empfehlung für dieses Vorgehen ist als Reaktion auf die (medial als solche bekannt gewordenen) Ausbrüche zu verstehen und bis dato nicht evidenzbasiert. INSIST untersucht, ob die Zielsetzungen des Screenings, i. Ausbrüche zu verhindern und ii. Infektionserreger vorherzusagen, erzielt werden. Darüber hinaus überprüft INSIST negative Effekte wie einen gesteigerten oder besonderen (Breitspektrum-)Antibiotikaeinsatz bei Nachweis resistenter kolonisierender Erreger

Patienten und Methodik: Eingeschlossen werden alle Früh- und Reifgeborenen, die zwischen 2011 und 2019 geboren und in der Universitätsmedizin Göttingen (UMG) innerhalb ihres ersten Lebensmonats stationär behandelt wurden. Screening-Ergebnisse gemäß KRINKO-Empfehlung bis maximal zum sechsten Lebensmonat wurden analysiert. Die Erregernachweise erfolgten mit klassischen kulturellen Methoden inklusive Resistenztestung, Resistenzgennachweisen und für Q4/2017-Q2/2020 zusätzlich mittels molekularer Feintypisierung (whole genome sequencing). Für den Zeitraum Q4/2011-Q3/2017 wurden alle im Rahmen des neonatologischen Kolonisationsscreenings erhobenen Befunde hinsichtlich der folgenden drei Fragestellungen evaluiert: i) Positiv-prädiktiver Wert (PPV) einer Kolonisation für spätere Infektionen, ii) Kolonisationsdynamik und iii) PPV für Häufung klonaler Stämme.

Ergebnisse: In den Jahren 2011 bis 2017 konnten insgesamt 3134 Patienten mit 44.689 Screeninguntersuchungen eingeschlossen werden, davon 30.930 mit Erregernachweis. Bislang fanden sich 2450 MSSA, 21 MRSA, 25 3MRGN E. coli, 63 3MRGN Klebsiella pneumoniae, 1663 Vancomycin-sensible und 40 Vancomycin-resistente Enterokokken. In dieser Zeit gab es bei 1727 Patienten insgesamt 2171 Episoden mit Antibiotikagabe. Zusammenfassung und Schlussfolgerung: Der Nachweis oder das Fehlen relevanter kolonisierender Erreger werden aktuell hinsichtlich Infektionsprädiktion untersucht. Es wird evaluiert, inwieweit über einen willkürlich gelegten Schwellenwert hinausgehende Kolonisationsnachweise eine beginnende Häufung/einen Ausbruch vorhersagen können (speziesspezifisch).

Durch die molekulare Feintypisierung kann eine Präzisierung der Hypothese der Stammidentität erreicht werden und die Rate der im Rahmen des neonatologischen Kolonisationsscreenings als falsch-positiv ,identisch" charakterisierten Isolate und folglich der falsch-positiven Alerts beziffert werden.

Des Weiteren wird der Antibiotikaverbrauch (auch einzelner Substanzen/ Substanzklassen) mit der vorausgegangenen Dichte an resistenten Erregern assoziiert. Alle Auswertungen sind aktuell gestartet und befinden sich in Bearbeitung.

\section{DGSPJ-PO 14 \\ Neugeborenenscreening auf endokrine und metabolische Erkrankungen in der europäischen Region der WHO}

Pia Maier', Susanne Carai' ${ }^{2}$, Aigul Kuttumuratova ${ }^{3}$, Martin Willi Weber ${ }^{3}$ 'Zentrum für Kinder- und Jugendmedizin, Universitätsklinikum Heidelberg, Klinik Kinderheilkunde I, Heidelberg, Deutschland; ${ }^{2}$ Universität Witten/ Herdecke, Globale Kindergesundheit, Witten, Deutschland; ${ }^{3}$ World Health Organization, Division of noncommunicable diseases and promoting health through the life-course, Child and Adolescent Health Programme, Kopenhagen, Dänemark

Hintergrund und Fragestellung: Entscheidungen über die in Neugeborenen-Screening-Programmen (NBSP) flächendeckend getesteten endokrinen und metabolischen Erkrankungen werden von jedem Land separat getroffen. Ein Konsens in der europäischen Region über den Umfang existiert nicht. Ziel des Projektes war die Analyse der NBSP in der durch die WHO definierten europäischen Region und der Position Deutschlands im europäischen Vergleich.

Material und Methode: In einem zweistufigen Verfahren wurde zunächst eine Online-Recherche hinsichtlich der nationalen NBSP durchgeführt. Waren keine bzw. keine aktuellen Informationen des Gesundheitsministeriums, der Krankenhäuser o. Ä. verfügbar, wurden erfahrene Ärzte aus dem jeweiligen Land als repräsentative Informanten befragt.

Ergebnisse: Informationen zu dem NBSP waren für 49 der 53 Länder und für den Kosovo verfügbar. Die Anzahl der Erkrankungen der NBSP liegt sich zwischen 0 und 40. In Deutschland sind derzeit 16 Erkrankungen im NBSP enthalten. Die am häufigsten in den NBSP repräsentierten Erkrankungen sind die kongenitale Hypothyreose (46 von 50) und die Phenylketonurie (44 von 50). In Albanien, Kirgistan und im Kosovo wird kein reguläres Neugeborenenscreening auf endokrine oder metabolische Erkrankungen durchgeführt.

Diskussion: Angeborene endokrine und metabolische Erkrankungen können erhöhte Morbidität und Mortalität bedingen. Durch eine frühe Identifikation und Behandlung können Folgeschäden reduziert bzw. verhindert 
und die Lebensqualität der Kinder verbessert werden. Dennoch besitzen nicht alle Länder ein nationales NBSP. Wichtig für die Interpretation der NBSP ist: 1. Das offiziell vorgesehene NBSP lässt keinen Rückschluss auf den Prozentsatz der tatsächlich untersuchten Neugeborenen zu. Insbesondere in ländlichen Regionen oder Ländern mit geringer Anzahl an Krankenhäusern wird eine schlechte Screening-Abdeckung vermutet. 2. Bei der Interpretation der eingeschlossenen Erkrankungen müssen landesspezifische Krankheitsinzidenzen berücksichtigt werden. 3. Bei Ländern, in denen nur auf wenige oder keine Erkrankungen flächendeckend getest wird, sollte bei Forderung nach Aufbau oder Erweiterung des NBSP neben dem politischen Gefüge auch das Gesundheitssystem und die Finanzierung von Gesundheitsleistungen hinterfragt werden. Denn ein Screening wäre nur dann ethisch vertretbar, wenn ein mit Erkrankung diagnostiziertes Kind auch eine adäquate Therapie erhalten würde.

Schlussfolgerung: Es bestehen große Unterschiede in den nationalen NBSP in der europäischen Region. Zielführend sind flächendeckende, evidenzbasierte Screening-Programme, die den positiven Nutzen für die Gesundheit der Neugeborenen im Fokus haben. Essentiell ist hierfür auch eine höhere Transparenz hinsichtlich der Screening-Abdeckung, nicht jedoch unweigerlich die Maximierung der Anzahl eingeschlossener Erkrankungen.

Empfehlungen für die europäische Region könnten helfen, Veränderungen in nationalen Leitlinien und Gesundheitssystemen zu initiieren.

\section{DGSPJ-PO 15}

\section{Einsatz von Webcams auf neonatologischen Intensivstationen - Implementierung und Evaluation (Neo-CamCare)}

Nadine Scholten', Sebastian Bretthauer', Martin Hellmich', Hendrik Hillen ${ }^{4}$, Dirk Horenkamp-Sonntag ${ }^{5}$, Christiane Jannes ${ }^{6}$, Ludwig Kuntz ${ }^{7}$, Andreas Müller', Christina Samel', Indra Spiecker', Stefanie Wobbe-Ribinski', Christiane Woopen ${ }^{6}$, Till Dresbach ${ }^{8}$

'Uniklinik Köln, Universität zu Köln, IMVR - Institut für Medizinsoziologie, Versorgungsforschung und Rehabilitationswissenschaft, Köln, Deutschland;'²Goethe-Universität Frankfurt, Lehrstuhl für Öffentliches Recht, Informationsrecht, Umweltrecht, Verwaltungswissenschaften, Frankfurt, Deutschland; ; Universität zu Köln, IMSB, Köln, Deutschland;'Universität zu Köln, MIG, Köln, Deutschland:5Techniker Krankenkasse, Versorgungsmanagement, Hamburg, Deutschland; ${ }^{6}$ Universität zu Köln, Ceres, Köln, Deutschland;'Universität zu Köln, MIG, Köln, Deutschland; ${ }^{8}$ Uniklinik Bonn, Neonatologie, Bonn, Deutschland; ${ }^{9}$ DAK, Versorgungsforschung, Hamburg, Deutschland

Hintergrund: Für Eltern frühgeborener Kinder besteht nicht immer die Möglichkeit, dauerhaft auf der neonatologischen Intensivstation (NICU) bei ihrem Frühgeborenen anwesend zu sein. Diese Trennung kann weitreichende Folgen für die Mütter und die Bindung zwischen den Eltern und dem Kind haben. Die Trennung von Mutter und Kind kann zu Schwierigkeiten in der Entwicklung von Muttergefühlen, zu weniger Verantwortungsgefühl, Sensibilität und zu Schwierigkeiten bei der Versorgung mit Muttermilch führen [1]. Eine Möglichkeit, den Folgen der räumlichen Trennung von Neugeborenen und ihren Eltern zu begegnen, ist die Einführung von Webcams auf NICUs. Diese, über dem Bett des Kindes installierte Kamera, soll es den Eltern ermöglichen, auch über die Distanz Sichtkontakt zu ihrem Kind zu halten und eine aus der Perspektive der Eltern bessere Beziehung aufzubauen. Positive Effekte auf das medizinische Outcome der Neugeborenen werden indirekt z. B. über das gesteigerte Wohlbefinden der Eltern erwartet. Durch die gesteigerte Empfindung von Nähe zum Kind soll die Laktation und somit die Ernährung der Neugeborenen mit Muttermilch gefördert werden. Neben den positiven Auswirkungen der Webcams berichten Eltern aber auch von gesteigerten Ängsten, ausgelöst durch das Beobachten kritischer Situationen.

Fragestellung: Ziel der Neo-CamCare Studie ist es, die Implementierung und den Einsatz von Webcams auf neonatologischen Intensivstationen in Deutschland wissenschaftlich zu begleiten und die Effekte auf die Eltern, Pflegekräfte und ÄrztInnen systematisch zu erheben. Förderer: Innovationsfonds des G-Ba (FKZ: 01VSF18037)
Methode: Die Implementierung und der Einsatz von Webcams auf den NICUs von drei deutschen Universitätsklinika und einem weiteren Krankenhaus mit Level I NICU evaluiert. Die Effekte auf die Eltern werden im Rahmen einer randomisiert-kontrollierten Studie im Wartegruppen-Kontrolldesign erfasst werden. Mit einbezogen wird dabei eine noch zu entwickelnde Handlungshilfe für die Eltern, die ihnen den Umgang mit der Kamera erleichtern soll. Zeitgleich wird der Effekt (Mehraufwand, Disruptionen) für die Pflegenden erhoben, der mit dem Einsatz der Webcams verbunden sein kann. Flankierend findet die Erstellung eines Rechtsgutachtens zur Aufarbeitung datenschutzrechtlicher und arbeitsdatenschutzrechtlicher Fragen, wie auch die Erfassung von Implementierungshürden durch eine Befragung aller NICU Leitungen in Deutschland statt. Eltern von Frühgeborenen, die bei einer der kooperierenden Krankenkasse versichert sind werden in Bezug auf ihre Einstellung, wie auch zur Situation von-Eltern Frühgeborener allgemein befragt.

\section{Literatur}

1. Henderson J, Carson C, Redshaw M (2016) Impact of preterm birth on maternal well-being and women's perceptions of their baby: A population-based survey. BMJ Open 6(10):e12676

\section{DGSPJ-PO 16 \\ Deskriptive Analyse von Spontanberichten über Arzneimittelnebenwirkungen bei Kindern}

Sarah Leitzen', Diana Dubrall', Irmgard Toni', Antje Neubert ${ }^{3}$, Julia Sting ${ }^{2}$, Bernhardt Sachs ${ }^{2}$

'BfArM, Forschung, Bonn, Deutschland;'B Bundesinstitut für Arzneimittel und Medizinprodukte (BfArM), Forschung, Bonn, Deutschland;'3niversitätsklinikum Erlangen, Kinder- und Jugendklinik, Kinderklinik, Erlangen, Deutschland;

Hintergrund: Wirksamkeit und Sicherheit von Arzneimitteln werden vor ihrer Zulassung oft nur an Erwachsenen getestet. Daher werden viele Arzneimittel bei Kindern „off-label“ eingesetzt. Dies kann mit einem erhöhten Risiko für unerwünschte Arzneimittelwirkungen (UAW; „Nebenwirkungen") verbunden sein. UAW-Datenbanken enthalten eine Vielzahl von UAW-Berichten zu Kindern. Analysen dieser Berichte können relevante Informationen zur Erhöhung der Arzneimitteltherapiesicherheit bei Kindern liefern.

Fragestellung/Ziel: Das Ziel dieser Untersuchung im Rahmen des KiDSafe-Projektes war es, UAW-Berichte zu Kindern aus Deutschland deskriptiv zu analysieren.

Material und Methoden: Für den Zeitraum von 01.01.2000-28.02.2019 wurden 20.854 UAW-Spontanberichte, die für die Altersklassen 0 bis $<18$ Jahre für Deutschland in der Europäischen UAW-Datenbank EudraVigilance vorlagen, in Bezug auf die Zahl der Meldungen pro Jahr, Meldequellen, demografische Parameter, am häufigsten gemeldete UAW und als verdächtigt gemeldete Wirkstoffe analysiert.

Ergebnisse: Die Anzahl der gemeldeten Spontanberichte pro Jahr hat seit 2000 kontinuierlich zugenommen. Im Jahr 2018 hatte sich die Anzahl der Spontanberichte im Vergleich zum Vorjahr verdoppelt (1354 vs. 2633 Fälle).

12,2\% der Berichte wurden ausschließlich von Patienten und sogenannten Non-Health Care Professionals gemeldet. 86,5\% der Spontanberichte stammten ausschließlich von Health Care Professionals.

$33,7 \%$ der Spontanberichte entfielen auf die Altersgruppe 3 bis 11 Jahre und $39,7 \%$ auf die Altersgruppe 12 bis $<18$ Jahre (vgl. Altersgruppe 0-1 Monat $11,8 \%$ und 2 Monate-2 Jahre $14,9 \%$ ).

Die am häufigsten berichteten UAW waren Erbrechen, Nesselsucht, Atemnot, Übelkeit und Ausschlag.

Die am häufigsten als verdächtigt gemeldeten Arzneistoffe waren Methylphenidat und Ibuprofen (+Kombi).

$74,4 \%$ der 20.854 UAW Meldungen wurden gemäß rechtlicher Definition als schwerwiegend klassifiziert.

Diskussion: Die kontinuierliche Zunahme der gemeldeten Spontanberichte seit dem Jahr 2000 könnte, neben anderen Ursachen, auch auf Verschär- 
fungen der gesetzlichen Meldeverpflichtungen zurückzuführen sein. Bei der Interpretation der Ergebnisse müssen die bekannten Limitierungen des Spontanberichtsystems berücksichtigt werden.

Schlussfolgerung: Der hohe Anteil der als schwerwiegend klassifizierten Spontanberichte sowie die konstante Zunahme der gemeldeten Spontanberichte unterstreichen die Bedeutung der Arzneimitteltherapiesicherheit bei Kindern. Die Meldung von UAW und die Analyse dieser Meldungen kann die Kenntnisse über UAW bei Kindern erhöhen und Anlass zu gezielteren Untersuchungen sein.

Innovationsausschuss G-BA; Förderkennzeichen: 01NVF16021

\author{
Literatur \\ 1. Leitzen S et al.: KiDSafe - Verbesserung der Versorgung von Kindern und Jugend- \\ lichen mit Arzneimitteln durch Erhöhung der Arzneimitteltherapiesicherheit \\ Bulletin zur Arzneimittelsicherheit 2018; 4: 24-27 \\ 2. Dubrall $D$ et al Häufige Nebenwirkungen.
}

\section{DGSPJ-PO 17}

Comprehensive assessments and related interventions to enhance long-term outcome in children, adolescents and young adults cancer survivor-Presentation of the CARE for CAYA program

Tineke Boesten', Barbara Koch', Gabrielle Eschrich', Stefan Bielack', G Calaminus ${ }^{5}$, Thorsten Langer ${ }^{6}$, CNiemeyer $^{7}$, D Reinhardt ${ }^{8}$, Alexander Stein ${ }^{8}$, Carsten Bokemeyer

'UKE-Hamburg, pädiatrische Hämatologie und Onkologie, Hamburg, Germany; ${ }^{2}$ UKE-Hamburg, Onkologie, Hamburg; ${ }^{3}$ Olgahospital Stuttgart, PHO, Stuttgart, Germany; ${ }^{4}$ Universitätsklinikum Bonn, PHO, Bonn, Germany; ${ }_{5}^{5}$ Universitätsklinikum Schleswig-Holstein, Campus Lübeck, PHO, Lübeck, Germany; ${ }^{6}$ Universitätsklinikum Freiburg, Zentrum für Kinder- und Jugendmedizin, PHO, Freiburg, Germany; ${ }^{7}$ Universitätsklinikum Essen, $\mathrm{PHO}$, Essen, Germany; ${ }^{8}$ UKE-Hamburg, Onkologie, Hamburg, Germany

Background: Improved, multimodal treatment strategies increase cure rates in cancer patients. Those who survive cancer as a child, adolescent or young adult (CAYA) are at high risk for therapy- or disease-related late or long-term effects. The CARE for CAYA-Program (CFC-P) has been developed to comprehensively assess potential future problems, to offer needbased preventive interventions and thus to improve long-term outcomes in this particularly vulnerable population.

Methods: The CFC-P is designed as an adaptive trial with an annual com prehensive assessment followed by need-stratified, modular interventions, currently including physical activity, nutrition and psychooncology, aimed to improve lifestyle and/or the psychosocial situation. Patients between 15 and 39 years with a prior cancer diagnosis, who have completed tumour therapy, are in follow-up care and are tumour free will be included. At baseline (and afterwards yearly) the current medical and psychosocial situation and lifestyle will be assessed by a survey compiled of validated questionnaires (e.g. EORTC QLQ C30, NCCN distress thermometer, PHQ-4 BSA, nutrition protocol) and objective parameters (e.g. BMI, WHR, comorbidities like hyperlipidaemia, hypertension or diabetes), followed by basic care (psychological and lifestyle consultation). Depending on their needs, CAYAs will be allocated to preventive interventions in the abovementioned modules (physical activity, nutrition and psychooncology) over a 12-month period. After one year, the assessment will be repeated and further interventions may be applied as needed. During the initial trial phase, the efficacy of this approach will be compared to standard care (waiting list with intervention in the following year) in a randomized study. During this phase, 530 CAYAs will be included and 320 randomized. Overall, 1500 CAYAs will be included and assessed. The CFC-P is financed by the innovation fund of the German Federal Joint Committee and will be conducted at 14 German sites.

Results: In January 2018 recruitment started at 14 german sites. As per February 2019340 patients were enrolled, 246 of which fulfilled the criteria's to be randomised. 76 patients did not show any specific needs in either of the three modules at baseline assessment and therefor did not qualify for randomisation.
Of the 119 Patients recruited in Hamburg, 85 showed a specific need for at least one module: 41 were randomised into interventional group and were distributed as follows: 32 patients within the psychooncological module, 21 patients in nutrition and 19 for the sport module

Discussion: CAYAs are at high risk for long-term sequelae, e. g. cardiac diseases or psychosocial problems. Providing structured interventions to improve lifestyle and the psychological situation may compensate these risk factors. The CFC-P serves to establish uniform regular comprehensive assessments and need-based interventions to improve long-term outcome in CAYA survivors.

\section{DGSPJ-PO 18}

\section{Aktuelle Trends in der Anwendung systemischer Antibiotika im pädiatrischen Setting - eine populationsbasierte deutschlandweite Studie}

Jakob Holstiege, Annika Steffen, Maike Schulz, Manas Akmatov, Jörg Bätzing Zentralinstitut für die kassenärztliche Versorgung in der Bundesrepublik Deutschland, Regionalisierte Versorgungsanalysen und Versorgungsatlas, Berlin, Deutschland

Zielsetzung: Bestimmung deutschlandweiter und regionaler krankenkassenübergreifender zeitlicher Trends der ambulanten Anwendung systemischer Antibiotika im pädiatrischen Setting für die Jahre 2010-2018.

Material und Methoden: Die Untersuchung erfolgte auf Basis einer Vollerfassung ambulanter Arzneiverordnungsdaten und unter Einschluss aller GKV-Versicherten unter 15 Jahren. Als Kennzahl für die Anwendung systemischer Antibiotika wurde die Verordnungsrate, d.h. die Anzahl von Antibiotikaverordnungen pro 1000 Versicherte im Jahr für die Jahre 2010-2018 bundesweit und auf Ebene der KV-Bereiche bestimmt. Zeitliche Trends wurden sowohl altersgruppenspezifisch als auch -übergreifend anhand von Poisson Regressionmodellen getestet.

Ergebnisse: Mit 683 Verordnungen pro 1000 Versicherte im Jahr 2018 konnte die mit Abstand höchste Verordnungsrate in der Altersgruppe der 2-5-Jährigen beobachtet werden (0-1 Jahr: 320, 6-9 Jahre: 417, 10-14 Jahre: 273). Insgesamt ging die Anwendungshäufigkeit systemischer Antibiotika in der ambulanten Versorgung bei Kindern unter 15 Jahren über den Studienzeitraum um $43 \%$ zurück $(p<0,001)$. Mit einer Reduktion von nahezu $50 \%$ wies die Altersgruppe der 0-1-Jährigen den stärksten Rückgang der Verordnungsrate auf $(p<0,001)$. Ein deutlich rückläufiger Verordnungstrend konnte zudem in allen KV-Bereichen und für die überwiegende Mehrheit der eingesetzten Wirkstoffgruppen beobachtet werden. Regional variierte die Verordnungsrate im Jahr 2018 um den Faktor 1,9 zwischen dem KV-Bereich mit dem höchsten (Saarland: 609) gegenüber dem mit dem niedrigsten Wert (Sachsen: 322).

Diskussion: Während Deutschland im europäischen Vergleich traditionell einen sehr niedrigen altersgruppenübergreifenden ambulanten Antibiotikagebrauch aufweist, zeigten sich noch im Jahr 2010 vergleichsweise hohe Verordnungsraten im pädiatrischen Setting. Die beobachteten deutlichen Reduktionen markieren einen Wandel in den pädiatrischen Verordnungsmustern und sind denkbares Resultat zahlreicher Initiativen zur Stärkung eines sachgerechten Antibiotikaeinsatzes in Deutschland. Starke Unterschiede zwischen den KV-Bereichen unterstreichen die Bedeutung regional zugeschnittener Programme in diesem Kontext.

Schlussfolgerung: Substanzielle Rückgänge des ambulanten Antibiotikaeinsatzes in der vertragsärztlichen Versorgung von Kindern und Jugendlichen deuten auf einen Paradigmenwechsel in Form eines zunehmend zurückhaltenden und rationalen Einsatzes systemischer Antibiotika in Deutschland hin. 
DGSPJ-PO 19

Verbesserung der Versorgung von Kindern- und Jugendlichen mit Arzneimitteln durch Erhöhung der Arzneimitteltherapiesicherheit - Das KiDSafe Projekt

Irmgard Toni', Dorothée Malonga Makosi ${ }^{2}$, Christine Gräf ${ }^{2}$, Philipp

Mildenberger ${ }^{2}$, Jochem König' ${ }^{2}$, Wolfgang Rascher', Michael S. Urschitz ${ }^{2}$, Antje

Neubert ${ }^{1}$

'Universitätsklinikum Erlangen, Kinder- und Jugendklinik, Erlangen, Deutschland;'Institut für Medizinische Biometrie, Epidemiologie und Informatik Universitätsmedizin der Johannes Gutenberg-Universität Mainz, Abteilung für Pädiatrische Epidemiologie, Mainz, Deutschland

Hintergrund: Kinder und Jugendliche sind bei der Arzneimittelversorgung benachteiligt. Durch off-label Anwendungen, fehlende pädiatrische Darreichungsformen und komplexe Dosisberechnungen treten Medikationsfehler (MF) im Vergleich zu Erwachsenen bis zu dreimal häufiger auf [1]. Internationale Studien zeigen, dass 3-5 \% aller stationären Aufnahmen aufgrund einer unerwünschten Arzneimittelwirkung (UAW) erfolgen [25]. Das bestehende Versorgungsdefizit in der päd. Arzneimitteltherapie soll durch die Einführung der neuen Versorgungsform PaedPharm verbessert und die Rate an MF und UAW gesenkt werden.

Methode: PaedPharm ist eine Qualitätssicherungsmaßnahme, die evidenzbasierte Informationen sowie spezifische Handlungsempfehlungen zur Anwendung von Arzneimitteln bei Kindern für ambulant tätige Ärzte zur Verfügung stellt. Es besteht aus drei Modulen: ein digitales Kinderarzneimittelinformationssystem, päd.-pharm. Qualitätszirkel und ein päd. UAW-Meldesystem. Es wird in 12 geographischen Clustern randomisiertkontrolliert und schrittweise in einem Stepped-Wedge-Design implementiert. Ein Cluster besteht aus einer Klinik für Kinder- und Jugendmedizin sowie ca. 20 pädiatrischen Praxen im Einzugsbereich. Der Anteil von UAW-bedingten stationären Aufnahmen unter allen nicht-elektiven Aufnahmen wird kontinuierlich vor und nach Einführung von PaedPharm ermittelt. Unter Berücksichtigung von Datenschutzvorgaben werden die UAW/MF-Verdachtsfälle aus den Kliniken an ein Referenzzentrum oder an die Arzneimittelkommission der deutschen Ärzteschaft (Akd̈̈) zur Validierung und Beurteilung übermittelt. Sekundäre Endpunkte umfassen die Zufriedenheit mit der Intervention, das Nutzungsverhalten und das Verordnungsverhalten der teilnehmenden Ärzte.

Ergebnisse: Die Erfassung des primären Endpunktes startete am 1. Juli 2018. Bis 30. April 2019 konnten 134 Ärzte für die Teilnahme gewonnen und PaedPharm in 6 von 12 Clustern eingeführt werden. In den 12 erhebenden Kliniken wurden bisher 17.000 nicht-elektive Fälle erfasst. Davon wurden 835 UAW-Verdachtsfälle an die zuständigen Bewertungsstellen übermittelt. Die Ergebnisse der Bewertungen stehen noch aus. Die Rückmeldung aus den Qualitätszirkeln ist durchweg positiv.

Diskussion: Bei der Implementierung von PaedPharm zeigte sich eine schleppende spontane Rekrutierung von niedergelassenen Ärzten. Diese wurde einer Ursachenanalyse zugeführt und entsprechende Gegenmaßnahmen eingeleitet. Die Inzidenz von UAW/MF bei stationärer Aufnahme kann anhand der vorliegenden Daten noch nicht geschätzt werden. Es ist zu erwarten, dass durch die Bündelung aller Aspekte der päd. Arzneimittelverordnung und -anwendung in praxisrelevanter und dennoch wissenschaftlich fundierter Form, eine nachhaltige Verbesserung aller patienten- und qualitätsrelevanten Parameter erreicht wird. Eine flächendeckende bundesweite Implementierung von PaedPharm und Aufnahme in die Regelversorgung ist angestrebt.

\footnotetext{
Literatur

1. Kaushal et al (2001) JAMA 285:2114-2120

2. Smyth et al (2012) PLoS ONE 7:e24061

3. Rashed et al (2012) Eur J Clin Pharmacol 68:801-810

4. Smyth et al (2014) Programme Grants Appl. Res 2:

5. Gallagher et al (2012) PLoS ONE 7:e50127
}

\section{Rehabilitation/Partizipation/Gesundheitsförderung/} Ausbildung

\section{DGSPJ-PO 20}

\section{Prädiktoren der Patientenzufriedenheit in der Kinder-Jugend- Rehabilitation - Vorstellung des Projektes PräZuKi}

Cindy Höhn', Gloria Metzner', Edith Waldeck', Matthias Stapel', Manuela Glattacker

'Universitätsklinikum Freiburg, Sektion Versorgungsforschung und Rehabilitationsforschung (SEVERA), Freiburg, Deutschland;'Edelsteinklinik, Fachklinik für Kinder- und Jugendrehabilitation, Bruchweiler; ${ }^{3}$ Deutsche Rentenversicherung Rheinland-Pfalz, Dezernat 3.3 Reha-Steuerung und -Strategie, Speyer, Deutschland

Hintergrund: Der medizinischen Rehabilitation kommt im Gesamtversorgungskonzept chronisch kranker Kinder und Jugendlicher eine hohe Bedeutung zu. Die Zufriedenheit von Kindern und Jugendlichen mit ihrer Rehabilitation ist insgesamt hoch. Dennoch gibt es auch Jugendliche, die mit ihrem Reha-Aufenthalt unzufrieden sind und die Maßnahme sogar vorzeitig abbrechen. Über die Einflussfaktoren auf die Patientenzufriedenheit und den Abbruch von Reha-Maßnahmen im Bereich der Kinder-Jugend-Rehabilitation ist wenig bekannt. Studien aus dem Erwachsenenbereich zeigen, dass subjektive Krankheits- und Behandlungskonzepte wichtige Prädiktoren der Zufriedenheit und anderer gesundheitsbezogener Outcomes sind.

Fragestellung: Ziel des Projektes PräZuKi ist es, Prädiktoren der Zufriedenheit von Jugendlichen mit ihrer Reha-Maßnahme sowie frühzeitiger Reha-Abbrüche zu identifizieren.

Material und Methoden: Die Studie setzt ein mixed-methods Design um. Ziel der qualitativen Vorstudie ist - v. a. unter Nutzung von Interview-Daten mit Jugendlichen - die Entwicklung eines Fragebogens zum rehabezogenen Behandlungskonzept Jugendlicher. Ziel der quantitativen Hauptstudie ist die Ermittlung von Prädiktoren der Patientenzufriedenheit und der Reha-Abbrüche. Die Hauptstudie hat ein prospektives längsschnittliches Eingruppen-Design mit 3 Messzeitpunkten (vor Reha-Beginn, 2 Wochen danach und Reha-Ende), und es sollen $\mathrm{N}=220$ Jugendliche zwischen 12 17 Jahren einbezogen werden. Als potenzielle Prädiktoren werden neben soziodemographischen und krankheitsbezogenen Variablen v.a. psychologische Konstrukte wie das subjektive Krankheits- und Behandlungskonzept der Jugendlichen untersucht.

Ergebnisse: Die qualitative Vorstudie ist abgeschlossen. Hierbei wurden zwischen Juli und September 2018 dreizehn Jugendliche zu ihren Vorstellungen, Erwartungen und Befürchtungen hinsichtlich ihres Reha-Aufenthaltes interviewt. Basierend auf den Ergebnissen einer qualitativen Inhaltsanalyse dieser Interviews und unter Nutzung von Items aus verwandten Fragebögen, wurden Fragebogen-Items zum rehabezogenen Behandlungskonzept von Jugendlichen formuliert. Diese Items wurden in einem kognitiven Pretest mit $\mathrm{N}=4$ Jugendlichen auf ihre Verständlichkeit hin geprüft und adaptiert und finden in dieser Form Anwendung in der im April 2019 begonnenen quantitativen Hauptstudie. Im Vortrag werden neben dem Studienprotokoll ausgewählte Ergebnisse der Vorstudie vorgestellt. Diskussion: Im Projekt PräZuKi wird ein Fragebogen zur Operationalisierung des rehabezogenen Behandlungskonzepts Jugendlicher entwickelt, der in zukünftigen Studien Anwendung finden kann. Darüber hinaus wird die Hauptstudie Erkenntnisse über Prädiktoren der Patientenzufriedenheit und frühzeitiger Reha-Abbrüche liefern, die letztlich in die Entwicklung konkreter Maßnahmen münden sollen, um die Zufriedenheit der Jugendlichen mit ihrer Rehabilitation zu steigern sowie das Risiko von Reha-Abbrüchen zu reduzieren. 
DGSPJ-PO 21

\section{Chronische Kopfschmerzen bei Schulkindern - 5-Jahre Follow-up eines multimodalen ambulanten Therapie-Programms}

Raymund Pothmann, Maite Hartwig

Delfin Kids - Zentrum für integrative Kinderschmerztherapie, Hamburg, Deutschland

Chronische Kopfschmerzen bei Schulkindern stellen eine zunehmende therapeutische Herausforderung dar. Die meisten Untersuchungen beschränken sich auf kleine Gruppen und beschäftigen sich überwiegend mit eindimensionalen Verfahren wie der medikamentösen Prophylaxe der Migräne. Erst in den letzten Jahren lässt sich ein internationaler Trend erkennen, der sich auf funktionelle Aspekte der Kopfschmerzen bezieht und damit über die reinen Kopfschmerzparameter hinausgeht. Die Übertragung in die Basisversorgung fehlt bisher völlig.

Methodik: 4000 Kinder und Jugendliche im Alter von 4-18 Jahren mit chronischen Kopfschmerzen wurden einem multimodalen Behandlungskonzept unterzogen. Nach einer einstündigen Befragung, Untersuchung und Beratung erfolgten weitere Behandlungseinheiten im Monatsrhythmus. Dabei wurden neben Lebensstilberatung verhaltensmedizinischen Verfahren, niedrig dosiertem Amitriptylin bei Dauerkopfschmerzen mit Schlafstörung und TENS eine lösungsfokussierte Strategie (Steve de Shazer \& Inso Kim Berg) angewandt. Ein und fünf Jahre nach Beendigung der Therapie erfolgte eine schriftliche Befragung einer Serie von 300 konsekutiv behandelten Kindern und Jugendlichen mit chronischen Schmerzen, 259 davon mit Kopfschmerzen.

Ergebnisse: 259 Kindern und Jugendlichen mit chronisch-rezidivierenden Kopfschmerzen wurden erfasst. Bei 28 \% der Kinder bestanden zwei Kopfschmerzdiagnosen. An Komorbiditäten fanden sich Bauchschmerzen, Rückenschmerzen und Gelenkschmerzen. Das durchschnittliche Alter zum Zeitpunkt des Erstkontaktes lag bei 11 Jahren. Die durchschnittliche Erkrankungsdauer betrug 3,2 Jahre, die Schmerzstärke: 6;1/10 auf der NRS PedMIDAS: 2,8/4 im Sinne einer deutlichen Lebensqualitätseinschränkung. Chronifizierungsgrad: 2/3. 2,4 Schultage und 3,8 Freizeitaktivitäten fielen/Monat aus. An 2,2 Tagen/Monat wurden Analgetika eingenommen. Bei einem Aufwand von 3,8 Therapieterminen innerhalb von 3,9 Monaten betrug die durchschnittliche Besserung $71 \%$, die nach 1 und 5 Jahren stabil blieb. Der Rücklauf der schriftlichen Nachbefragung lag bei 57 \%. Die Lebensqualität, ablesbar an der Reduktion der Kopfschmerzfrequenz und -intensität, sowie der sozialen Ausfallstage und des Analgetikaverbrauchs nahm signifikant zu

Diskussion und Fazit: Die Ergebnisse einer pragmatischen ambulanten Kopfschmerztherapie sind sowohl effektiv als auch ökonomisch. Die Lebensqualitätseinbuße bzw. Chronifizierung ist mit anderen Untersuchungen vergleichbar. Der Vorteil der systemischen Rahmenbedingungen liegt im Ressourcen fokussierten Behandlungsansatz. Bisherige Untersuchungen beschränkten sich oft nur auf ein Krankheitsbild (z. B. Migräne) oder Einzelverfahren. Die Kombination aus Lebensstilberatung, Schmerzedukation, Entspannung und gezielten weiteren Therapiemodulen richtet sich nach dem inviduellen Bedarf und stellt eine konkret umsetzbare Chance für die Bewältigung der defizitären Versorgungssituation von Kindern und Jugendlichen mit chronischen Kopfschmerzen dar.

\section{Literatur}

1. De Shazer S (1990) Wege der erfolgreichen Kuzzeittherapie. Klett-Cotta, Stuttgart

2. Eccleston C et al (2002) Systematic review of randomised controlled trials of psychological therapy for chronic pain in children. Pain 99:157-165
DGSPJ-PO 22

\section{Kopf-, Bauch- und Rückenschmerzen bei Kindern und} Jugendlichen in Deutschland (KiGGS Welle 2 2014-2017)

Hannelore Neuhauser', Roma Thamm², Giselle Sarganas', Laura Krause ${ }^{2}$ 'Robert Koch-Institut; Deutsches Zentrum für Herz-Kreislauf-Forschung Standort Berlin, Abteilung für Epidemiologie und Gesundheitsmonitoring, Berlin, Deutschland;'Robert Koch-Institut Berlin, Abteilung für Epidemiologie und Gesundheitsmonitoring, 12101 Berlin, Deutschland

Hintergrund: Schmerzen sind ein wichtiges Thema für die Gesundheit von Kindern und Jugendlichen: Sie führen nicht nur zu individuellem Leidensdruck sondern auch häufig zu Schulfehlzeiten, Medikamenteneinnahme und Inanspruchnahme ärztlicher Leistungen. Schmerzen im Kindesalter sind zudem assoziiert mit Schmerzen im Erwachsenenalter.

Methoden: Basierend auf Daten der bundesweiten KiGGS Welle 2 (20142017) werden wiederholt auftretende Kopf-, Bauch- und Rückenschmerzen in den letzten 3 Monaten bei Mädchen und Jungen berichtet und mit Daten der KiGGS-Basiserhebung (2003-2006) verglichen. Folgen wiederholt auftretender Kopfschmerzen werden ebenfalls untersucht. Für die Analysen standen aus KiGGS Welle 2 Daten von $>11.000$ Teilnehmenden und aus der Basiserhebung von > 12.000 Teilnehmenden zwischen 3 und 17 Jahren zur Verfügung. Für 3- bis 10-jährige Kinder beantworteten die Eltern/Sorgeberechtigten die Fragen, während 11- bis 17-Jährige selbst Auskunft gaben.

Ergebnisse: Bei 3- bis 10-Jährigen kommen wiederholt auftretende Bauchschmerzen am häufigsten vor und betreffen ein Drittel der Mädchen und ein Viertel der Jungen. Bei 11- bis 17-Jährigen sind wiederholt auftretende Kopfschmerzen führend und betreffen fast jedes zweite Mädchen und etwa jeden dritten Jungen. Die 3-Monats-Prävalenz wiederholt auftretender Kopf-, Bauch- und Rückenschmerzen hat bei Mädchen und Jungen vor allem in den Altersgruppen 7 bis 10 Jahre und 11 bis 13 Jahre zugenommen. Jugendliche nehmen bei wiederholt auftretenden Kopfschmerzen fast doppelt so oft Medikamente ein wie Kinder.

Diskussion: Kopf-, Bauch- und Rückenschmerzen sind nach wie vor und mit z.T. steigender Prävalenz sehr häufige Symptome bei Kindern und Jugendlichen in Deutschland. Ihre Prävention benötigt einen ganzheitlichen Blick auf die Kindergesundheit im psychosozialen, Lebensumfeldund Versorgungskontext.

\section{DGSPJ-PO 23}

\section{Alle spielen mit! Sinn und Unsinn der differenzierten Schulsportbefreiung}

Alexander Heimann', Swantje Scharenberg' ${ }^{2}$, Christoph Wechsler ${ }^{3}$, Oliver Muensterer', Tatjana König'

'Universitätsmedizin Mainz, Klinik für Kinderchirurgie, Mainz, Deutschland; ${ }^{2}$ Karlsruher Institut für Technologie, Forschungszentrums für den Schulsport und den Sport von Kindern und Jugendlichen, Karlsruhe, Deutschland; ${ }^{3}$ Schweizerischer Verband für Sport in der Schule (SVSS), SVSS Weiterbildung, Binningen, Schweiz

Fragestellung: Die WHO empfiehlt täglich $1 \mathrm{~h}$ körperlicher Aktivität für Kinder und Jugendliche. Schüler/innen mit körperlichen Einschränkungen in den Sportunterreicht einzubeziehen, stellt jedoch eine Herausforderung für Ärzte und Sportlehrkräfte dar. „Activdispens“ ist ein multimediales System zur angepassten Bewegung für Kinder und Jugendliche mit körperlichen Einschränkungen im Schulsport, das in der Schweiz bereits angewendet wird. Diese Studie untersucht, wie oft Ärzte eine differenzierte Sportbefreiung ausstellen und welche Probleme bei der praktischen Umsetzung bestehen.

Methode: Onlineumfrage unter Ärzten und Sportlehrkräften aus Deutschland und der Schweiz.

Ergebnisse: Die befragten Ärzte $(n=71)$, waren zu $86 \%$ Pädiater oder Kinderchirurgen, 52 \% im niedergelassen Bereich. Sie gaben an, Sportbefreiungen am häufigsten aufgrund von akuten Verletzungen (76 \%) oder Infektionen (42\%) auszustellen. Chronische Erkrankungen stellen selten einen Grund dar (14\%). Nur 59 \% haben schon einmal eine differenzier- 
te Sportbefreiung ausgestellt, $18 \%$ hatten noch nie darüber nachgedacht. Eine differenzierte Sportbefreiung fanden $90 \%$ sinnvoll, aber nur $59 \%$ gleichzeitig umsetzbar.

Sportlehrkräften aus Deutschland $(n=197)$ gaben an, dass $11,5 \%$ der Schüler nicht am Sportunterricht teilnehmen und meist (90\%) von den Eltern befreit werden. Gründe waren neben vergessener Sportkleidung (60\%) Infekte (57\%) und Verletzungen (50\%). Chronische Erkrankungen waren selten ein Grund (7 \%). Eine differenzierte Sportbefreiung hielten $92 \%$ für sinnvoll, aber nur $58 \%$ für sinnvoll und umsetzbar.

Sportlehrkräften der schweizer „Activdispens“-Schulen, $(n=180)$ verwenden „Activdispens“ in $60 \%$ d.F. konsequent und $32 \%$ manchmal. Die Nichtteilnehmer-Quote beim Sport war niedriger $(8 \%)$ als in deutschen Schulen. Die Entschuldigung erfolgte häufiger ärztlich und seltener durch die Eltern (34 \%). 60 \% der Lehrer berichten jedoch, dass eine differenzierte Sportbefreiung vom Arzt selten oder nie vorgelegt wurde. Eine differenzierte Sportbefreiung fanden $85 \%$ der Lehrer sinnvoll, 58 \% umsetzbar. Diskussion: Ärzte und Lehrer sind sich einig, dass eine differenziert Sportbefreiung sinnvoll ist. Die praktische Umsetzung stellt sich jedoch schwierig dar. Viele Ärzte haben sich noch nie mit dem Thema beschäftigt, bestehende Vorlagen für eine differenzierte Sportbefreiung werden kaum verwendet. In den "Activdispens“-Schulen nahmen prozentual mehr Schüler am Sportunterricht teil.

Schlussfolgerung: Kinder und Jugendliche mit körperlichen Einschränkungen profitieren von einer differenzierten Sportbefreiung. Diese wird jedoch nur selten umgesetzt. Ärzte und Lehrkräfte sollten für das Thema sensibilisiert sein und Patienten nach Möglichkeit eine angepasste Teilnahme am Sportunterricht im Rahmen ihrer körperlichen Voraussetzungen ermöglichen. Die systematische Umsetzung erfordert eine gute interdisziplinäre Kooperation. Standardisierte Systeme wie „Activdispens“ können hier die Kommunikation erleichtern.

\section{DGSPJ-PO 24}

\section{Vorverlegung der Schuleingangsuntersuchung: Können Kinder mit Förder- oder Therapiebedarf zuverlässig identifiziert werden? Ergebnisse aus dem Pilotprojekt GESiK (Gesundheits- und Entwicklungsscreening im Kindergartenalter) in der Landeshauptstadt München}

\section{Anna Katharina Meyer-Borgstädt, Claudia Sulser, Doris Wohlrab, Kathrin} Martignoni

Referat für Gesundheit und Umwelt, Landeshauptstadt München, Sachgebiet Schulgesundheit, München, Deutschland

Nach dem Beschluss des Bayerischen Ministerrats vom Sommer 2014 soll die Schuleingangsuntersuchung in Bayern neu konzipiert werden. Ziel der Reform ist die Anpassung des Gesundheits- und Entwicklungsscreenings an den aktuellen wissenschaftlichen Standard der Entwicklungsdiagnostik Der Untersuchungszeitpunkt soll um ein Jahr in das vorletzte Kindergartenjahr vorverlegt werden. So soll bestehender Förder- oder Therapiebedarf frühzeitig festgestellt und mehr Zeit für die Umsetzung der entsprechenden Maßnahmen vor der Einschulung gewonnen werden.

Das Referat für Gesundheit und Umwelt (RGU) der Landeshauptstadt München hat sich mit fünf weiteren Landkreisen und Städten im Pilotprojekt GESiK unter Leitung des Bayerischen Landesamtes für Gesundheit und Lebensmittelsicherheit (LGL) an der Neukonzeption beteiligt. Seit Januar 2016 werden pro Jahr ca. 800 Münchner Kinder mit dem neuen Entwicklungs- und Gesundheitsscreening untersucht.

Hierbei erfolgt für jedes Kind zunächst ein Screening durch die Gesundheits- und Kinderkrankenpflegerinnen und -pfleger (GKKP). Bei auffälligen Ergebnissen wird im Anschluss eine ärztliche Untersuchung durchgeführt. Bei Bedarf kann eine zweite Untersuchung im Jahr vor der Einschulung erfolgen.

Ziel des Pilotprojektes war es, das neue Entwicklungs- und Gesundheitsscreening auf Durchführbarkeit, Effektivität, Akzeptanz und Nutzen für die auf freiwilliger Basis teilnehmenden Kinder zu evaluieren.

Das RGU München hat zusätzlich zu diesen Zielen einen weiteren Schwerpunkt auf die zuverlässige Identifikation aller Kinder mit Förder- und The- rapiebedarf zu dem früheren Untersuchungszeitpunkt gelegt. Alle Kinder durchliefen sowohl das Screening als auch die ärztliche Untersuchung. Im Folgejahr wurde dieses Regime für die bereits einmal untersuchten Kinder wiederholt, die Ergebnisse wurden miteinander verglichen.

Für beide Untersuchungszeitpunkte konnten Daten von insgesamt 406 Kindern ausgewertet werden. Es konnte bestätigt werden, dass alle Kinder mit Förder- und Therapiebedarf im neuen Gesundheits- und Entwicklungsscreening bereits zu dem frühen Untersuchungszeitpunkt zuverlässig erfasst werden können.

Die reformierte Schuleingangsuntersuchung kann potentiellen Förderund Therapiebedarf zuverlässig und frühzeitig erkennen. Eine anschließende ärztliche Untersuchung ist nur bei auffälligem Screening erforderlich und sinnvoll.

\section{DGSPJ-PO 25}

\section{Wissen und Einstellungen von Ergotherapeuten, Logopäden und Physiotherapeuten zur Erkrankung Epilepsie}

Katharina Hackel', Martina Patrizia Neininger' ${ }^{2}$, Wieland Kiess', Thilo Bertsche ${ }^{2}$, Astrid Bertsche ${ }^{3}$

'Universitätsklinikum Leipzig, Klinik und Poliklinik für KInder- und Jugendmedizin, Leipzig, Deutschland; '2Universität Leipzig, Zentrum für Arzneimittelsicherheit und Klinische Pharmazie, Leipzig, Deutschland; ${ }^{3}$ Universitätsmedizin Rostock, Kinder- und Jugendklinik; Universitätsklinikum Leipzig, Klinik für Kinder- und Jugendmedizin, Bereich Neuropädiatrie; Klinik und Poliklinik für Kinder- und Jugendmedizin, Rostock/Leipzig, Deutschland

Fragestellung: Ergotherapeuten, Logopäden und Physiotherapeuten spielen eine entscheidende Rolle in der Behandlung von Patienten mit Epilepsie. Dies gilt besonders für Kinder und Jugendliche in Kindergarten und Schule. Zu Wissen und Einstellungen von Mitgliedern dieser Berufsgruppen ist jedoch wenig bekannt.

Material und Methode: Nach Vorliegen eines positiven Ethikvotums führten wir eine Erhebung zu Epilepsie bei Ergotherapeuten, Logopäden und Physiotherapeuten aus Leipzig und dem Landkreis Zwickau mittels Fragebogen durch.

Ergebnisse: An der Befragung nahmen 195 Therapeuten (T) teil, 74 (38 \%) Ergotherapeuten (E), 58 (30 \%) Logopäden (L), 63 (32 \%) Physiotherapeuten (P). Insgesamt hatten sich $92 \%$ der T bereits mit dem Krankheitsbild beschäftigt (E: 97 \%, L: 95 \%, P: 84 \%). Bei 65 \% wurde Epilepsie während der Ausbildung (E: 78 \%, L: $62 \%$, P: $52 \%$ ) thematisiert. Von allen T gaben $8 \%$ an, noch keine Epilepsiepatienten behandelt zu haben (E: $9 \%$, L: $3 \%$, P: $11 \%$ ).

Mit einem epileptischen Anfall waren in ihrer Berufsausübung $43 \%$ der T bereits konfrontiert (E: $46 \%$, L: 45 \%, P: 38 \%). Von allen T wussten $44 \%$ (E: 38 \%, L: $45 \%$, P: $49 \%$ ), dass man bei einem Krampfanfall unter ungünstigen Bedingungen sterben kann.

Bei einem Anfall würden $42 \%$ der T einen festen Gegenstand in den Mund schieben (E: 31 \%, L: 31 \%, P: 63 \%). Ein ärztlich verordnetes Notfallmedikament würden $41 \%$ der T verabreichen (E: $51 \%$, L:31 \% P: 38 \%). Bei Präsentation eines Fotos von buccalem Midazolam gaben $8 \%$ der T an, das Medikament zu kennen (E: 7 \%, L: 9 \%, P: $10 \%$ ). Rektales Diazepam kannten 41 \% der T (E: 36 \%, L:41 \%, P 46 \%). Rechtliche Konsequenzen eines Fehlverhaltens bei einer Notfallarzneimittelgabe fürchten $55 \%$ der T (E: 59 \%, L: 64 \%, P: 43 \%). Mehr Informationen zum Thema Epilepsie wünschen sich $92 \%$ der T (E: $88 \%$, L: $91 \%$, P: $97 \%$ ).

Diskussion: Die meisten Therapeuten behandeln Patienten mit Epilepsie und knapp die Hälfte ist während der Berufsausübung bereits mit einem epileptischen Anfall konfrontiert gewesen. Dennoch gaben nur zwei Drittel der Teilnehmer an, dass die Erkrankung in der Ausbildung thematisiert worden sei, bei den Physiotherapeuten sogar nur die Hälfte. Weniger als die Hälfte der Befragten nimmt richtigerweise an, dass man bei einem Krampfanfall unter ungünstigen Bedingungen sterben kann. Knapp die Hälfte der Teilnehmer würde fälschlicherweise bei einem Krampfanfall einen festen Gegenstand in den Mund schieben, diese Fehlannahme ist insbesondere bei Physiotherapeuten noch verbreitet. Weniger als die Hälfte 
der Befragten würde bei einem Krampfanfall ein ärztlich verordnetes Notfallarzneimittel verabreichen. Trotz der hohen Zahl an behandelten Epilepsiepatienten sind die beiden derzeit für die Laiengabe zugelassenen Epilepsienotfallmedikamente den meisten Therapeuten nicht bekannt. Fast alle Teilnehmer wünschen sich mehr Informationen zum Thema Epilepsie. Schlussfolgerung: Auf Basis der erhobenen Daten erscheinen Schulungen der Therapeuten zum Thema Epilepsie dringend erforderlich.

\section{DGSPJ-PO 26}

\section{Weiterbilden, wo die Versorgung von Kindern und Jugendlichen stattfindet}

\section{Christoph Weiß-Becker', Ralf van Heek2, Georg Hillebrand ${ }^{3}$}

'Gemeinschaftspraxis, Kinder- und Jugendmedizin, Husum, Deutschland;'Praxis für Kinder- und Jugendmedizin, Praxis, Altenholz, Deutschland; ${ }^{3}$ Klinikum Itzehoe, Klinik für Kinder- und Jugendmedizin Itzehoe, Deutschland

Einleitung: In der medizinischen Versorgung erleben wir eine sich zuspitzende Problematik bei der Sicherung der Bedarfe. Die ambulante Grundversorgung in der Fläche wird schwieriger, auch in der Pädiatrie, wo klinische und ambulante Versorgung gleichermaßen wichtig sind. Wer Versorgung für morgen sichern will, muss heute weiterbilden. Die kinderund jugendärztliche Rolle erlernen diejenigen, die strukturiert und kompetenzbasiert die Inhalte an den Orten der Versorgung vermittelt bekommen. Eine gute Weiterbildung lehrt Pädiatrie sektorenübergreifend mit guten Weiterbildungskonzepten.

Fragestellung: Wie kann pädiatrische Weiterbildung in Kliniken und Praxen umgesetzt werden, damit sie die erforderlichen Kompetenzen hochwertig vermittelt? Welche Bedeutung hat dabei ein kompetenzbasiertes We iterbildungscurriculum?Material und Methoden: In Schleswig-Holstein haben sich die Kliniken der akut versorgenden Pädiatrie und ein Netz von Weiterbildungspraxen unter Moderation durch den BVKJ zum Weiterbildungsverbund Pädiatrie zusammengeschlossen. Folgende Rahmenbedingungen wurden vereinbart: Abordnungsvertrag zwischen Klinik, Praxis und Weiterzubildenden; Fortbestand des Weiterbildungsvertrages; Weiterbildung in der ambulanten Grundversorgung mit halber Stelle bevorzugt im 3. und 4. Weiterbildungsjahr; Schicht- und Bereitschaftsdienste in der Klinik; verschiedene Arbeitszeitmodelle werden unter den Beteiligten abgestimmt. Die Weiterbildung in der Praxis erfolgt nach dem strukturierten, kompetenzbasierten und in elektronischer Form hinterlegten Weiterbildungscurriculum PaedCompenda ${ }^{\circ}$ der DGAAP (Deutsche Gesellschaft für ambulante allgemeine Pädiatrie).

Ergebnisse: In den letzten 3 Jahren wurden mehrere Verbundverträge zwischen Kliniken und Praxen geschlossen, die Weitergebildeten haben die Erfahrungen in der ambulanten Pädiatrie positiv bewertet.

Zusammenarbeit zwischen Kliniken und Praxen in der Weiterbildung schafft gegenseitige Kenntnis und Vertrauen und hilft beim Abbau von Vorurteilen über die Tätigkeiten und Kompetenzen des Anderen. Die Durchlässigkeit der Grenzen kann die Interessen und die Bedeutung der Pädiatrie stärken. Die vielfältigen Arbeitsfelder und Versorgungsstrukturen der ambulanten pädiatrischen Grundversorgung neben der Klinik erfahrbar zu machen, kann helfen, das Fach attraktiver zu machen. Es braucht einen langen Atem, um tragfähige Kooperationsstrukturen aufzubauen. Erfreulich ist ein hohes Interesse seitens der Niedergelassenen und der Kliniker.

Zusammenfassung: Der Weiterbildungsverbund pädiatrischer Kliniken und Praxen in Schleswig-Holstein etabliert eine sektorenübergreifende und kompetenzbasierte Facharztweiterbildung unter Einsatz eines digitalen Weiterbildungscurriculums. Interessante Weiterbildungskonzepte im Rahmen der Zusammenarbeit von Kliniken und Praxen können die vielseitige, anspruchsvolle und attraktive Tätigkeit in der Kinder- und Jugendmedizin darstellen.

\section{DGSPJ-PO 27}

\section{Kindzentrierte Psychosoziale Grundversorgung im Ambulanten Sektor (KID-PROTEKT)}

\section{Nikola Nitzschke', Silke Pawils², Sönke Siefert ${ }^{1}$}

'Katholisches Kinderkrankenhaus Wilhelmstift gGmbH, Stiftung

Familienorientierte Nachsorge Hamburg SeeYou, Hamburg, Deutschlan d; ${ }^{2}$ Universitätsklinikum Hamburg-Eppendorf, Zentrum für Psychosoziale Medizin, Institut und Poliklinik für Medizinische Psychologie, Hamburg, Deutschland

Hintergrund: Im Vergleich zur Normalbevölkerung sind die Risiken für Kinder aus psychosozial oder gesundheitlich hoch belasteten Familien, eine schwere, chronische oder psychische Krankheit oder gravierende Entwicklungsstörungen zu erleiden, deutlich erhöht. Pränataler Stress und frühkindliches Erleben von psychosozialen Belastungen können demzufolge lebenslange gesundheitliche Konsequenzen haben. Innerhalb der Frühen Hilfen und der Kinder- und Jugendhilfe gibt es zahlreiche Unterstützungsangebote für Familien mit psychosozialen Belastungen. Häufig kommen jedoch gerade Familien mit intensivem Unterstützungsbedarf nicht im Hilfesystem an bzw. nehmen unterstützende Angebote nur unzureichend in Anspruch.

Fragestellung: KID-PROTEKT untersucht, wie die systemübergreifende Zusammenarbeit von ambulanter Gynäkologie bzw. Pädiatrie mit der Kinder- und Jugendhilfe zur Umsetzung der gesetzlich geforderten Präventionsleistungen unter Effektivitäts- und Effizienzaspekten bestmöglich gelingen kann. Ziel ist die Förderung einer gesunden Kindesentwicklung trotz psychosozialer Belastungssituation in der Familie.

Die bestehende Regelversorgung zur Schwangerenvorsorge bzw. Früherkennung von Krankheiten bei Kindern und Jugendlichen wird um eine psychosoziale Belastungsanamnese erweitert. Durch das standardisierte Erfassen von Belastungen können Familien mit Unterstützungsbedarf systematisch erkannt und an adäquate Hilfen weitervermittelt werden. Das medizinische Fachpersonal wird in der Früherkennung psychosozialer Belastungen geschult und die Praxis über eine bessere Vernetzung entlastet. Methodik: In einem clusterrandomisierten Kontrollgruppenvergleich gegenüber „Treatment as usual“ (TAU) wird ein Verfahren, bei dem eine sozialpädagogische Fachkraft eine Sprechstunde in der Praxis durchführt und die Lotsenfunktion zu den Hilfsangeboten übernimmt („Supported Treatment") mit einer Variante ohne Lotsin („Qualified Treatment") verglichen. Die teilnehmenden Frauen- bzw. Kinder- und Jugendarztpraxen werden randomisiert den Bedingungen zugeordnet und der psychosoziale Unterstützungsbedarf der Patienten erhoben. Primäre Outcomes sind Effektivität und Effizienz im Vergleich zur aktuellen Versorgung. Dazu werden die Bedarfsquote im Verhältnis zur Belastungsquote, die Weiterleitungsquote im Verhältnis zur Bedarfsquote und die Häufigkeit der Inanspruchnahme gemessen. Als sekundäre Outcomes werden Akzeptanz und Machbarkeit mittels Patienten- und Praxenbefragung ermittelt und die Generalisierbarkeit u. a. durch Analyse regionaler Einflüsse geprüft. Eine Nicht-Teilnehmer-Analyse gibt Aufschluss über die Gründe der Teilnahmeverweigerung zu unterschiedlichen Zeitpunkten der Versorgung.

Ergebnisse: Das Projekt läuft seit September 2018 und wird für drei Jahre durch den Innovationsfonds des Gemeinsamen Bundesausschusses gefördert. Aktuell werden die teilnehmenden Arztpraxen rekrutiert und je nach Interventionsarm geschult. Die Umsetzung in den Praxen beginnt im September 2019. 


\section{DGSPJ-PO 28}

\section{Partizipative Entscheidungsfindung mit Patienten von 7-18 Jahren und ihren Eltern: Entwicklung eines Instruments und erste empirische Ergebnisse}

Michael Eichinger', Angelique Herrler', Tatiana Görig', Sabine Georg', Heike Philippi' ${ }^{2}$ Freia De Bock ${ }^{3}$

${ }^{1}$ Medizinische Fakultät Mannheim der Universität Heidelberg, Mannheimer Institut für Public Health und Klinik für Kinder- und Jugendmedizin, Mannheim, Deutschland;'2Sozialpädiatrisches Zentrum Frankfurt-Mitte, vae, Leitung, Frankfurt, Deutschland; ${ }^{3}$ Medizinische Fakultät Mannheim der Universität Heidelberg, Mannheimer Institut für Public Health und Klinik für Kinder- und Jugendmedizin, Mannheimer Institut für Public Health, Mannheim, Deutschland

Hintergrund: Patientenzentrierte Endpunkte in der Pädiatrie sind wichtig Oft allerdings fehlen valide Messinstrumente. Die partizipative Entscheidungsfindung (PEF) mit Patienten und Eltern ist ein solcher Endpunkt. Fragestellung: Ziel der Studie war es, für den deutschen Sprachraum erstmals (1) Skalen zur Erfassung des Grades an PEF mit Patienten von 7-18 Jahren und ihren Eltern zu entwickeln und (2) erste psychometrische Eigenschaften zu untersuchen.

Methoden: Auf Basis der englischen CollaboRATE-Skala zur Messung der PEF mit erwachsenen Patienten entwickelten wir durch kognitive Interviews und iterative Überarbeitungen PEF-Skalen für (i) minderjährige Patienten, (ii) elterliche Fremdauskünfte zur PEF mit Patienten sowie (iii) elterliche Selbstauskünfte (höhere Werte bedeuten mehr PEF). In die Pilotierung des Fragebogens an 2 Sozialpädiatrischen Zentren wurden 230 Patienten-Elternpaare eingeschlossen. In der psychometrischen Evaluation wurden Crohnbach's alpha und bivariate Korrelationen zwischen Eltern- und Patienteneinschätzung analysiert.

Ergebnisse: Die 3 Originalitems mussten im Rahmen der kognitiven Interviews angepasst werden (z. B. umfassender Gesundheitsbegriff, Texthervorhebungen), um die Verständlichkeit zu erhöhen. In rund $57 \%$ der elterlichen Selbstauskünfte, 45 \% der elterlichen Fremdauskünfte und $37 \%$ der Patientenangaben wurde die PEF als optimal eingestuft. Die interne Konsistenz der Skalen war sehr gut (Alpha: 0,87-0,92). Es zeigte sich ein starker Zusammenhang zwischen Patienten- und elterlichen Fremdangaben $(r=0,87, p<0,001)$.

Diskussion und Schlussfolgerungen: Mit den 3 PEF-Skalen stehen in der Pädiatrie erstmals ökonomisch einsetzbare deutschsprachige Instrumente zur Erfassung der PEF mit Kindern und ihren Eltern zur Verfügung. Falls Kinder selbst keine Angaben zur PEF machen können (z. B. kognitive Beeinträchtigung), stellt die elterliche Fremdauskunft eine Alternative dar. Weiterführende psychometrische Analysen (z. B. Kriteriumsvalidität) sind geplant.

\section{Chronische Erkrankungen 1}

\section{DGSPJ-PO 29}

PRO-Kind-Rheuma - Handlungs- und Therapie-Protokolle in der Kinderrheumatologie

Klaus Tenbrock', Kirsten Minden², Dirk Föll', Gerd Horneff

'Uniklinik RWTH Aachen, Pädiatrie, Aachen, Deutschland;'2DRFZ Berlin, Epidemiologie, Berlin, Deutschland; ${ }^{3}$ Universitätsklinikum Münster, Pädiatrische Rheumatologie und Immunologie, Münster, Deutschland; ${ }^{4}$ Asklepios Klinik Sankt Augustin, Allgemeine Pädiatrie, St. Augustin, Deutschland

In Deutschland sind ca. 20.000 Kinder und Jugendliche unter 18 Jahren von entzündlichen Erkrankungen der Muskeln und Gelenke (muskuloskelettale Erkrankungen=MSE) betroffen. Über die Hälfte der Betroffenen bleibt bis ins Erwachsenenalter behandlungsbedürftig. Viele haben ein hohes Risiko dauerhaft körperlich deutlich eingeschränkt zu sein. Häufig werden diese Patienten zu spät oder unangemessen therapiert.
Für die juvenile idiopathische Arthritis (Gelenkrheuma) sowie für potenziell besonders einschränkende Bindegewebserkrankungen (z. B. die juvenile Dermatomyositis) haben die bundesweit tätigen Kinder- und Jugendrheumatologen standardisierte Handlungs- und Therapieprotokolle erstellt. Im Projekt PRO-Kind-Rheuma werden kinderrheumatologische Behandlungsteams motiviert, diese Protokolle anzuwenden. Dafür werden sie entsprechend geschult. Die Behandlung und Therapie von jungen MSEPatienten wird protokolliert. Die routinemäßige Rückmeldung der Patienten hilft dabei, die Therapie individuell auf die Erkrankten anzupassen. An zwanzig Standorten wird die medizinische Versorgung von etwa 500 neu erkrankten MSE-Patienten im ersten Behandlungsjahr beobachtet und analysiert. Es wird überprüft, ob die Behandlungsprotokolle im klinischen Alltag angewendet werden und das therapeutische Vorgehen an den verschiedenen Einrichtungen dadurch harmonisiert wird. Die Wirksamkeit der in den Protokollen vorgeschlagenen verschiedenen Therapiestrategien wird vergleichend geprüft. Das Projekt wird für drei Jahre mit insgesamt rund einer Million Euro gefördert. Die Erkenntnisse fließen wiederum in die Protokolle ein, sodass die Versorgung und letztendlich die Prognose rheumakranker Kinder und Jugendlicher langfristig weiter verbessert wird.

\section{DGSPJ-PO 30}

\section{Zufriedenheit von Kindern und Jugendlichen mit chronischen Erkrankungen in der Schule - Online-Befragung von Schüler*innen mit chronisch entzündlichen Darmerkrankungen}

Sina Krone', Sarah Adelmann', Dominik de Sordi', Martin Classen², Antje Ballauff', Gisela Schulze ${ }^{4}$, Antje Timmer ${ }^{1}$

${ }^{1}$ Carl von Ossietzky Universität Oldenburg, Epidemiologie und Biometrie, Oldenburg, Deutschland;'Klinikum Links der Weser, Klinik für Kinder- und Jugendmedizin, Bremen, Deutschland; ${ }^{3} \mathrm{HELIOS}$ Klinikum Krefeld, Zentrum für Kinder- und Jugendmedizin, Krefeld, Deutschland; ${ }^{4}$ Carl von Ossietzky Universität Oldenburg, Institut für Sonder- und Rehabilitationspädagogik, Fakultät für Bildungs- und Sozialwissenschaften, Oldenburg, Deutschland

Fragestellung: Chronisch entzündliche Darmerkrankungen (CED) schränken betroffene Kinder und Jugendliche in ihrem Alltag z. T. erheblich ein. Eine vorangegangene Befragung ergab, dass die Zufriedenheit der Eltern mit der ärztlichen Versorgung relativ hoch ist, trotz gewisser Einschränkungen. Dagegen äußerten Eltern hohe Unzufriedenheit mit dem Umgang mit dem erkrankten Kind in der Schule. Diese Befragung untersucht im Rahmen eines Qualifikationsprojektes, welche Aspekte des Schulalltages für Schüler mit CED von besonderer Relevanz sind und welche konkreten Mängel oder auch Wünsche genannt werden.

Methode: Im Zeitraum zwischen Januar 2018 und Februar 2019 wurden Schüler*innen im Alter von 10 bis 17 Jahren mit Morbus Crohn, Colitis ulcerosa oder unklassifizierbarer CED über zwei regional diverse CED-Ambulanzen sowie soziale Netzwerke kontaktiert und online befragt. Der Fragebogen war auf der Basis semistrukturierter Interviews mit Experten und Betroffenen erstellt worden. Zur Erhebung der Schulzufriedenheit und der gesundheitsbezogenen Lebensqualität wurden eine Subdomäne des YHCSUN (Range: 1,00 bis 5,00) und das EQ-5D-Y-Instrument verwendet. Ergebnisse: 63 Fragebögen konnten in die Auswertung eingeschlossen werden. 27 Schüler (42,9\%) gaben bzgl. des Verlaufes der CED in den letzten 12 Monaten an, mehrere Schübe oder andauernd Probleme aufgrund der Erkrankung gehabt zu haben; 36 Schüler (57,1 \%) äußerten einige bis große Probleme mit Niedergeschlagenheit und Angst (Gesundheitsprofil EQ-5D-Y). Der Mittelwert des EQ-VAS-Score betrug 73,0 (SD: 20,95).

Der Median des Schulzufriedenheits-Scores lag bei 2,50 (IQR: 1,67-3,63); 23 Schüler (36,5\%) beschrieben die Sauberkeit der Schultoiletten als sehr problematisch. Die Teilnahme an Klassenfahrten war für 14 Schüler $(22,2 \%)$ nur selten oder nicht möglich. Von 18 Schülern, die angaben, kaum Unterstützung beim Nachholen verpassten Unterrichtsstoffes durch ihre Lehrer zu erhalten, äußerten sechs Schüler, dies als sehr problematisch zu empfinden.

Diskussion: Von hoher Relevanz für Schüler mit CED erweisen sich in dieser Befragung die strukturelle Ausstattung der Schulen, Teilnahmen an 
Klassenfahrten sowie das Nachholen verpassten Lernstoffes. Neben symptombezogenen Schwierigkeiten scheinen interpersonelle Faktoren, wie die Kommunikation der Erkrankung in der Schule von Bedeutung zu sein. Unzufriedenheit mit sanitären Anlagen in Schulen sowie geringe Schulzufriedenheitswerte stimmen mit Befunden aus der Literatur überein. Für die Implementierung von Maßnahmen zur Verbesserung der Situation ist die Einbindung von Schulen und Lehrern wichtig.

Schlussfolgerung: Der Handlungsbedarf in Schulen bei der Unterstützung chronisch kranker Schüler wird durch geringe Schulzufriedenheitswerte betont. Die Befragung dient als Vorbereitung einer Befragung von Lehrern. Ziel ist die Identifikation von Ansatzpunkten für eine gezielte Verbesserung der derzeit als problematisch eingestuften Situation von Kindern mit CED in der Schule.

\section{DGSPJ-PO 31}

\section{Verbesserung der Versorgung für Kinder und Jugendliche mit chronisch entzündlichen Darmerkrankungen CED KQN - medizinische Bedeutung und Versorgung}

Jan de Laffolie', Jan Däbritz' ${ }^{2}$ Klaus-Peter Zimmer ${ }^{3}$, Keywan Sohrabi ${ }^{4}$, Michael Radke, Melanie Görsch ${ }^{6}$, Nanja van den Berg ${ }^{6}$, Stefan Trenkel ${ }^{5}$, Nicolas Schneider', Philipp Krieb ${ }^{4}$, Henning Schneider', CEDATA Study Group ${ }^{3}$

'Zentrum für Kinderheilkunde der Justus-v.-Liebig-Universität, Allgemeine Pädiatrie, Gießen, Deutschland;'Universitätsmedizin Rostock, Kinder- und Jugendklinik, Kindergastroenterologie, Rostock, Deutschland; ${ }^{3} J u s t u s-$ Liebig Universität Gießen, Allgemeine Pädiatrie und Neonatologie,

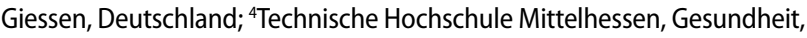
Giessen, Deutschland; ${ }^{5}$ Klinikum Westbrandenburg, Standort Potsdam, Kindergastroenterologie, Potsdam, Deutschland;‘Universitätsklinikum Greifswald, Community Medicine, Greifswald, Deutschland; ' Justus-Liebig Universität Gießen, Medizinische Informatik, Giessen, Deutschland

Fragestellung: Chronisch entzündliche Darmerkrankungen bei Kindern und Jugendlichen spielen eine zunehmende Rolle mit steigender Inzidenz sowie schwieriger Versorgungssituation mit hoher Morbidität und Mortalität. Durch standardisierte Dokumentation von Diagnostik- und Behandlungsdaten sollen Erkenntnisse über diese Patientenpopulation gewonnen werden, um Ansätze zur Verbesserung der Versorgung abzuleiten.

Material und Methoden: Eingebracht in das Projekt wurde das seit 2004 bestehende Patientenregister CEDATA GPGE. Es wurden Bedarfe zur Verbesserung der Versorgung und Identifikation von Versorgungsdefiziten in Arbeitspaketen zusammengestellt und in Kooperation der beteiligten Hochschulzentren umgesetzt.

Ergebnisse: 14 Arbeitspakete wurden als relevante Bausteine konzipiert Neben einer clusterrandomisierten Studie unter neu ins Register aufgenommenen Ambulanzen und den Datenvergleichen von Kindern und Jugendlichen mit CED innerhalb und außerhalb des Registers zur Identifikation von Versorgungsdefiziten, wurden die Arbeitspakete Anwendungsentwicklung, Data Management, IT Infrastruktur, Datensicherheit und Datenschutz adressiert sowie Projekte zu den Themen Transition und Patientenempowerment.

Diskussion: Das CEDKQN Projekt untersucht strukturiert die Versorgungssituation von Kindern und Jugendlichen mit CED in Deutschland. Es soll auch untersucht werden, ob externe Qualitätssicherung und -verbesserung zu einer höheren Versorgungsqualität beitragen kann.

\section{DGSPJ-PO 32}

Verbesserung der Versorgung von Kinder und Jugendliche mit chronisch entzündlichen Darmerkrankungen CED KQN - IT und Methoden

Jan de Laffolie', Jan Däbritz², Klaus-Peter Zimmer ${ }^{3}$, Keywan Sohrabi', Michael Radke ${ }^{5}$, Melanie Görsch ${ }^{6}$, Nanja van den Berg ${ }^{6}$, Stefan Trenkel', Nicolas Schneider', Philipp Krieb ${ }^{4}$, Henning Schneider', CEDATA Study Group ${ }^{3}$

'Zentrum für Kinderheilkunde der Justus-v.-Liebig-Universität, Allgemeine Pädiatrie, Gießen, Deutschland;'²Universitätsmedizin Rostock, Kinder- und Jugendklinik, Kindergastroenterologie, Rostock, Deutschland; ${ }^{3}$ JustusLiebig Universität Gießen, Allgemeine Pädiatrie und Neonatologie,

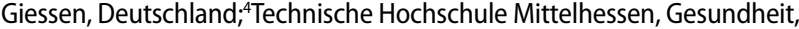
Giessen, Deutschland; ${ }^{5}$ Klinikum Westbrandenburg, Standort Potsdam, Kindergastroenterologie, Potsdam, Deutschland;'Universitätsklinikum Greifswald, Community Medicine, Greifswald, Deutschland; ${ }^{7}$ Justus-Liebig Universität Gießen, Medizinische Informatik, Giessen, Deutschland

Fragestellung: Chronisch entzündliche Darmerkrankungen bei Kindern und Jugendlichen spielen eine zunehmend wichtige Rolle mit steigender Inzidenz sowie schwieriger Versorgungssituation mit hoher Morbidität und Mortalität. Im Rahmen des CED-KQN Projekts soll basierend auf dem digitalen klinischen Register Cedata unter anderem eine clusterrandomisierte Studie durchgeführt werden, welche die Auswirkung von Echtzeitrückmeldungen bei der Datenerhebung untersucht sowie moderne Analyseverfahren (Big Data Techniken) zur Anwendung kommen. Material und Methoden: Eingebracht in das Projekt wurde das bestehende CEDATA GPGE Register, welches zur Umsetzung der Studie erheblich erweitert wurde. Dabei wurden moderne Webtechnologien verwendet, um die ortsunabhängige Datenerhebung unter Einhaltung aktueller Datensicherheitsstandards zu gewährleisten. In einem agilen Softwareentwicklungsprozess wurde in gemeinsamer Arbeit mit Expertengruppen ein Validationssystem zur Feedback-Ausgabe konzipiert.

Ergebnisse: Es wurden 31 IT - Projektteile als relevant für die clusterrandomisierte Studie identifiziert. Diese wurden drei Meilensteinen zugeordnet, die sequenziell bearbeitet wurden, wodurch die Teile voneinander unabhängig umgesetzt wurden. Die erweiterte Webapplikation kann somit Echtzeitrückmeldungen einblenden und diese sowie eventuelle Reaktionen des Nutzers auch speichern. Zur Umsetzung von Datenschutz und Datensicherheit wurde ein Rollen-Rechte-System implementiert, das Nutzern nur für deren Rolle notwendige Zugriffsrechte einräumt. Des Weiteren wurde ein Backup- und Wartungskonzept erstellt, welches Datenverlusten und Sicherheitslücken vorbeugen soll.

Diskussion: Die implementierten Funktionen können die Anforderungen von clusterrandomisierten Studien an das klinische Register Cedata erfüllen und ermöglichen so die webbasierte Datenerfassung mit Echtzeitrückmeldung. Diese Rückmeldungen können zwar auf eventuelle Versorgungsdefizite hinweisen, das Register muss jedoch klar von einem Medizinprodukt, welches bei Diagnostik und Therapie unterstützt, abgegrenzt werden. Dabei ist das Potential eines klinischen Registers damit noch nicht ausgeschöpft, es bieten sich weitere Funktionserweiterungen wie zum Beispiel Benchmarking und Datenschnittstellen zum Import und Export an.

Schlussfolgerung: Unter Verwendung agiler Entwicklungsmodelle ist es möglich, mit Webtechnologien ein klinikübergreifendes Forschungsregister mit Algorithmen basiertem Feedback zu implementieren, welches Studien zur Verbesserung der Versorgung chronisch erkrankter Kinder unterstützt. Der Betrieb des Registers erfordert dabei kontinuierliche Wartung und Weiterentwicklung. 


\section{DGSPJ-PO 33}

\section{TIC-PEA: telemedical interdisciplinary care for patients with esophageal atresia - ein vom innovationsausschuss des GBA gefördertes Projekt zur telemedizinischen Versorgung von Kindern mit Ösophagusatresie}

Oliver Muensterer, Jan Gödeke

Universitätsmedizin der Johannes Gutenberg-Universität Mainz, Klinik und Poliklinik für Kinderchirurgie, Mainz, Deutschland

Hintergrund: Das Ziel des TIC-PEA-Projekts ist es, die Versorgung von Kindern mit Ösophagusatresie gezielt und langfristig durch telemedizinische Beratung und Begleitung zu unterstützen. Inhalt es Projekts ist es daher, auf telemedizinischer Basis ein Netzwerk von Spezialisten zur Behandlung einer angeborenen Unterbrechung der Speisröhre (Ösophagusatresie) aufzubauen. Bislang existiert diese Art von interdisziplinärem, telemedizinischem Netzwerk, das lokale Behandler, Expertenvereinigung, Selbsthilfeorganisation, betroffene Patienten und deren Angehörige zusammenbringt, noch nicht. Das Netzwerk soll sich aus Patienten, Angehörige und behandelnden Ärzte, die in eine Studie zur Ösophagusatresie eingeschlossen werden, speisen.

Methodik: Die Zusammenarbeit in dem Netzwerk soll wie folgt ablaufen: Die Beteiligten erhalten einen Link zur Einwahl in die telemedizinische Plattform. Die erste telemedizinische Kontaktaufnahme erfolgt als virtuelles, koordiniertes Team-Treffen vor der operativen Versorgung der Ösophagusatresie zusammen mit Kinderchirurgen der Universitätsmedizin Mainz und Experten der Pediatric Adolescent and Adult Foregut Interdisciplinary Society (PAAFIS). Nach der Versorgung soll die Weiterbetreuung in zeitlich festgelegten telemedizinischen Sprechstunden erfolgen. Auswertung: Das Projekt wird durch das IMBEI (Institut für Medizinische Biometrie, Epidemiologie und Informatik) in seiner Evaluation und Auswertung begleitet. Dabei wird das in die Studie eingeschlossene Patientenkollektiv mit Krankenkassendaten von solchen Patienten, die nicht partizipieren, sowie historischen Daten verglichen. Die primären Outcome-Parameter dabei sind die Anzahl der Dilatation der Speiseröhre im ersten Lebensjahr. Sekundäre Outcome-Parameter sind unter anderem die Verweildauer im Krankenhaus, die Anzahl der Operationen bis zum Erreichen der Ösophaguskontinuität, die Zeit bis zur oralen Nahrungsaufnahme und die Mortalität.

Zusammenfassung: Diese neue telemedizinische Versorgungsform wird zum ersten Mal die akute und langfristige gezielte Betreuung der Patienten unter Einbeziehung aller relevanten Disziplinen umfassen, somit definiert sie einen neuen, einheitlichen Therapiestandard. Damit schafft sie potentiell die Grundlage einer optimierten Behandlung.

\section{DGSPJ-PO 34}

\section{Präventive Beratung zur Übergewichtsprävention: „GeMuKi - Gemeinsam Gesund: Vorsorge plus für Mutter und Kind“}

Franziska Krebs' ', Laura Lorenz', Farah Nawabi', Isabel Lück², Anne-Madeleine Bau', Adrienne Alayli', Stephanie Stock ${ }^{4}$

'Uniklinik Köln, Institut für Gesundheitsökonomie und Klinische

Epidemiologie, Köln, Deutschland;'Plattform Ernährung und Bewegung e.V., Innovationsfondsprojekt GeMuKi, Berlin, Deutschland; ${ }^{3}$ Plattform Ernährung und Bewegung e.V., Stuttgart, Deutschland; ${ }^{4}$ Universitätsklinikum Köln, Institut für Gesundheitsökonomie und Klinische Epidemiologie, Köln, Deutschland

Hintergrund: Das spätere Übergewichtsrisiko sowie das Risiko chronischer Erkrankungen (z. B. Diabetes mellitus) beim Kind werden bereits während der Schwangerschaft durch den mütterlichen Lebensstil beeinflusst (pränatale Programmierung). Auf Basis dieser Erkenntnisse wird eine individuelle präventive Lebensstilberatung in die regulären Vorsorgeuntersuchungen während der Schwangerschaft und im ersten Lebensjahr des Kindes integriert.

Fragestellung: GeMuKi ist eine komplexe Intervention, die aus mehreren Komponenten besteht und in Kooperation verschiedener Leistungserbringer (Frauenärzte, Hebammen und Kinderärzte) durchgeführt wird. Die
Evaluation beantwortet Fragen der Wirksamkeit, Wirtschaftlichkeit und des Implementierungsprozesses von GeMuKi.

Methode: GeMuKi ergänzt die Vorsorgeuntersuchungen in der Schwangerschaft und nach der Geburt durch eine strukturierte, niedrigschwellige Präventionsmaßnahme in den Bereichen Bewegung, Ernährung und Genussmittelkonsum. Ziele sind die Reduktion des Übergewichtsrisikos bei Mutter und Kind, die Stärkung der Gesundheitskompetenz werdender Mütter sowie die Vernetzung des medizinischen Fachpersonals.

GeMuKi wird seit Januar 2019 in 4 Regionen Baden-Württembergs implementiert. Teilnehmen können volljährige Schwangere. Teilnehmerinnen der Interventionsregionen erhalten zu 11 Zeitpunkten eine individuelle Beratung durch verschiedene Leistungserbringer in der Versorgungskette, während Teilnehmerinnen der 4 Kontrollregionen die Regelversorgung erhalten. Im Rahmen eines cluster-RCTs mit 2550 Teilnehmerinnen finden zu 4 Zeitpunkten Befragungen statt. Zusätzlich werden Gewichtsentwicklung und Gesundheitsdaten von Mutter und Kind zu jedem Vorsorgezeitpunkt erfasst. Anhand von GKV-Routinedaten wird die Inanspruchnahme von Gesundheitsleistungen untersucht. Im Rahmen der Prozessevaluation werden förderliche und hemmende Faktoren der Implementierung identifiziert.

Ergebnisse: Durch eine gezielte Präventionsmaßnahme mit Beginn der Schwangerschaft soll das Risiko für Übergewicht sowie chronische Erkrankungen reduziert werden.

Mit Abschluss der Studie in 2021 kann die Wirksamkeit, der Implementierungsprozess und die Wirtschaftlichkeit der neuen Versorgungsform bewertet werden.

Diskussion: GeMuKi trägt der Forderung nach langfristigen, niedrigschwelligen, multimodalen Interventionen mit Einbettung in Lebenswelten und bereits vorhandene Netzwerkstrukturen Rechnung. Die präventive Beratung im Rahmen der Vorsorgeuntersuchungen zielt auf die Reduktion des Übergewichtsrisikos, die Verbesserung der Gesundheitskompetenz und die Ausbildung gesundheitsförderlicher Verhaltensweisen ab. Darüber hinaus wird durch bessere Vernetzung und Schulung der Fachakteure in der Versorgungskette neben der Verhaltensprävention auf Personenebene auch die Verhältnisprävention auf Strukturebene berücksichtigt.

\section{DGSPJ-PO 35 \\ Was sind die Einflussfaktoren der Adipositas bei Mädchen und Jungen im Schulalter? Ergebnisse einer systematischen Literaturrecherche im Rahmen des AdiMon-Projektes.}

Gianni Varnaccia, Franziska Lehmann, Johannes Zeiher, Cornelia Lange, Susanne Jordan

Robert Koch-Institut, Epidemiologie und Gesundheitsmonitoring, Berlin, Deutschland

Fragestellung: Adipositas kann bereits im Kindes- und Jugendalter die Gesundheit beeinträchtigen und bis ins Erwachsenenalter negative gesundheitliche Folgen haben. In Deutschland ist etwa jedes sechste Kind übergewichtig oder adipös. AdiMon ist ein vom Robert Koch-Institut aufgebautes, webbasiertes Indikatorensystem, das systematisch zusammengestellte und regelmäßig aktualisierte Daten über die Einflussfaktoren der Adipositas bei Kindern liefert. Im Rahmen der Erweiterung der Zielgruppe vom Kita- (0-6 Jahre) auf das gesamte Kindes- und Jugendalter wurde eine systematische Literaturrecherche nach adipositasrelevanten Einflussfaktoren bei Mädchen und Jungen im Schulalter (7-17 Jahre) durchgeführt.

Material und Methode: Die Literaturrecherche erfolgte in den Datenbanken Scopus, PubMed, Embase, Web of Science und Cochrane. Berücksichtigt wurden systematische Übersichtsarbeiten zu adipositasrelevanten Einflussfaktoren bei Mädchen und Jungen im Schulalter. Die in den Übersichtsarbeiten identifizierten Einflussfaktoren wurden Einflussbereichen zugeordnet.

Ergebnisse: Die Suche ergab 2959 Treffer, von denen 279 Artikel die Einschlusskriterien erfüllten. Insgesamt wurden über 70 adipositasrelevante Einflussfaktoren identifiziert. Eine ausgewogene Ernährung, ein körper- 
lich aktiver Alltag und ausreichend Schlaf sind von zentraler Bedeutung für die Adipositasprävention. Das individuelle Verhalten wird maßgeblich von den Verhältnissen beeinflusst, in denen die Kinder aufwachsen. Hierzu gehören eine gesunde Verpflegung im häuslichen Umfeld und in der Schule sowie eine bewegungsfreundliche Wohnumgebung. Eine wichtige Rolle spielen auch die sozioökonomischen Rahmenbedingungen wie das Einkommen der Eltern oder deren Bildungsstand. Darüber hinaus sind biologische Faktoren wie die genetische Prädisposition, pränatale Faktoren wie das Rauchen der Mutter und frühkindliche Faktoren wie das Stillen wichtige Determinanten der Adipositas. Psychosoziale Faktoren können ebenfalls für die Entwicklung einer Adipositas relevant sein. Hierzu gehören protektive Faktoren wie ein ausgeprägtes Gesundheitsbewusstsein und Risikofaktoren wie bestimmte psychische Störungen (z. B. Depressionen). Nicht zuletzt können Maßnahmen der Prävention und Gesundheitsförderung einen wichtigen Beitrag leisten, um der Entwicklung von Adipositas bei Jungen und Mädchen im Schulalter vorzubeugen.

Schlussfolgerung: Die Ergebnisse der Literaturrecherche verdeutlichen, dass die Ursachen der Adipositas bei Mädchen und Jungen im Schulalter vielfältig sind und sich nicht auf eine ungesunde Ernährung und Bewegungsmangel reduzieren lassen. AdiMon bietet umfassende Informationen über die multifaktoriellen Ursachen der Adipositas bei Kindern und Jugendlichen. Diese können und sollen z. B. von Akteuren im Gesundheitswesen, im Bildungs- und Sozialbereich genutzt werden, um präventive Aktivitäten zu planen und umzusetzen.

\section{DGSPJ-PO 36 \\ PROMPt - Primärindikative und optimierte Zuweisung zu gezielten Maßnahmen bei emotionalen und Verhaltensauffälligkeiten bei Kindern}

\section{Cornelia Siegmund, Julia Ernst, Patricia Porst, Max Weniger}

TU Dresden, Institut für Klinische Psychologie und Psychotherapie, Dresden, Deutschland

Emotionale und Verhaltensprobleme beginnen häufig im Kindesalter mit oft weitreichenden Folgen für die soziale, emotionale und kognitive Entwicklung. Prävalenzschätzungen zu psychischen Auffälligkeiten in Deutschland zeigen, dass etwa 7,4 \% der drei- bis sechsjährigen und $7,8 \%$ der sieben- bis zehnjährigen von emotionalen (z. B. Rückzug, Ängstlichkeit, Depressivität) oder Verhaltensauffälligkeiten (z. B. oppositionelles Verhalten, Aggression, Hyperaktivität) betroffen sind.

Das vom GB-A Innovationsfond zur Förderung neuer Versorgungsformen geförderte PROMPt-Projekt hat zum Ziel eine optimierte Versorgungskette in die Routineversorgung zu implementieren. Dazu sollen Kinder mit einem erhöhten Risiko für die Entwicklung von emotionalen und Verhaltensstörungen direkt bei den Kinder- und Jugendärzten frühzeitig identifiziert und unmittelbar einem etablierten indizierten Präventionsprogramm zugewiesen werden. Dazu werden im Raum Dresden Eltern während der regulären Gesundheitsuntersuchungen (U-Untersuchungen, U9: 5-6 Jahre, U10: 7-8 Jahre, U11: 9-10 Jahre) gebeten den Strengths and Difficulties Questionnaire (SDQ; inklusive SDQ impact) auszufüllen, welcher u. a. Verhaltensauffälligkeiten und Stärken ihrer Kinder erfasst. Durch die selektivvertragliche Ausgestaltung erhalten die Eltern von ihrem Kinderarzt anschließend eine Rückmeldung und konkrete Empfehlungen zu möglichen Maßnahmen. Diese umfassen bei präventionsbedürftigen Kindern das Trainingsprogramm „Mutig werden mit Til Tiger“ für sozial unsichere Kinder und das Baghira-Training für häufig trotzige oder zornige Kinder. Geplant ist ein Screening von mehr als 3500 Kindern in ca. 50 Arztpraxen. Davon erhalten ca. 8 bzw. 15 \% der Kinder eine Empfehlung zur Teilnahme an einem der beiden genannten indikativen Präventionsprogrammen. Diese Kinder werden sowohl vor der Programmteilnahme, als auch nach Abschluss des Programms sowie 12 Monate später befragt. Unauffällige und hochauffällige Kinder, die an keinem der beiden Programme teilnehmen, werden zum Versorgungsverlauf ebenfalls 6 Monate und 12 Monate nach dem Screening für eine Fragebogenuntersuchung eingeladen. Durch Abstimmung der Erhebungsinstrumente und Messzeitpunkte mit der BELLA-Studienkohorte erfolgt zusätzlich ein Vergleich mit einer bundesweit repräsentativen unbehandelten Stichprobe. Erwartet werden disseminationsfähige evidenzbasierte Ergebnisse, die unmittelbar genutzt werden, um Präventionsangebote stärker in die Regelversorgung zu implementieren und so die persönliche Belastung von einzelnen Personen sowie gesellschaftliche Kosten infolge emotionaler und Verhaltensprobleme nachhaltig zu reduzieren.

\section{DGSPJ-PO 37 \\ Muster der Inanspruchnahme fachärztlicher Versorgung von Kindern und Jugendlichen mit ADHS in der KiGGS Welle 2}

\section{Robert Schlack, Stephan Junker}

Robert Koch-Institut Berlin, Epidemiologie und

Gesundheitsberichterstattung, Berlin, Deutschland

Fragestellung: Die Versorgung von Kindern und Jugendlichen mit ADHS erfolgt durch verschiedene Facharztgruppen, darunter nicht unwesentlich durch Kinder- und Jugendärzte. Mit den Daten der aktuellen KiGGS Welle 2 des Robert Koch-Instituts (Erhebungszeitraum 2014-2017) lassen sich Muster der fachärztlichen Inanspruchnahme bei ADHS für unterschiedliche Bevölkerungsgruppen untersuchen. Die Daten der KiGGS Welle 2 zeigten, dass es bezüglich diagnostizierter ADHS weiterhin einen deutlichen Sozialgradienten zuungunsten von Kindern aus Familien mit niedrigem Sozialstatus gibt (Göbel et al., 2018). Aus der KiGGS-Basiserhebung (2003-2006) ist zudem bekannt, dass ADHS bei Kindern mit Migrationshintergrund weniger häufig diagnostiziert wird (Schlack et al., 2007). Es wird der Frage nachgegangen, wie die fachärztliche Inanspruchnahme bei aktueller ADHS (12-Monats-Prävalenz) mit dem familiären Sozialstatus (SES) und einem familiären Migrationshintergrund assoziiert ist.

Material und Methoden: An dem bundesweit repräsentativen Untersuchungs- und Befragungssurvey zur Kinder- und Jugendgesundheit KiGGS Welle 2 (2014-2017) nahmen im Querschnitt insgesamt 15.023 Kinder und Jugendliche und ihre Eltern teil. Für die Analyse fachärztlicher Inanspruchnahme bei ADHS stehen Befragungsdaten von insgesamt 13.568 Teilnehmenden (6758 Jungen und 6810 Mädchen) im Alter von 3-17 Jahren zur Verfügung.

Ergebnisse: Insgesamt 4,3\% der Eltern berichteten für Ihre Kinder eine jemals durch einen Arzt oder Psychologen vergebene ADHS-Diagnose, bei insgesamt 2,3\% $(n=359)$ bestand die ADHS auch in den letzten 12 Monaten. Die Wahrscheinlichkeit elternberichteter ADHS war für Jungen dreimal höher als für Mädchen $(\mathrm{OR}=3,12)$, bei hohem SES mehr als doppelt so hoch wie bei niedrigem $(\mathrm{OR}=2,31)$ und bei Migrationshintergrund sehr viel geringer als ohne einen solchen $(\mathrm{OR}=0,18)$. Insgesamt nahmen 68,1 \% der Kinder mit ADHS in den letzten 12 Monaten einen Kinderarzt in Anspruch, 50 \% einen (Kinder)Psychiater und 18,2 \% einen Psychologen. Die Chance einer kinderärztlichen Inanspruchnahme war in der multivariaten, alters- und geschlechtsadjustierten Analyse bei Kindern mit niedrigem (vs. hohem) SES deutlich geringer $(\mathrm{OR}=0,20)$, bei Migranten gegenüber Nichtmigranten 11-fach erhöht $(\mathrm{OR}=11,1)$, diejenige der Inanspruchnahme eines (Kinder)Psychiaters $(\mathrm{OR}=0,27, \mathrm{n}$. s.) oder Psychologen $(\mathrm{OR}=0,01)$ dagegen deutlich niedriger als bei Nichtmigranten. Diskussion: Während Kinder mit ADHS aus Familien mit niedrigem Sozialstatus seltener in der Kinderarztpraxis vorgestellt werden als solche aus Familien mit hohem Sozialstatus, scheinen Kinderärzte für Familien mit Migrationshintergrund und Kindern mit ADHS eine Hauptanlaufstelle in der fachärztlichen Versorgung zu sein. Mögliche Inanspruchnahmebarrieren (kinder)psychiatrischer und -psychologischer Versorgung für Migranten wären zu diskutieren.

\section{Literatur}

1. Göbel K, Baumgarten F, Kuntz B, Hölling H, Schlack R (2018) ADHS bei Kindern und Jugendlichen in Deutschland - Querschnittergebnisse aus KiGGS Welle 2 und Trends. J Health Monit 3(3):46-53

2. Schlack, R., Hölling, H., Kurth, B. 
DGSPJ-PO 38

Entwicklung eines IT-gestützten Case Managements zur Verbesserung der Versorgungsqualität und Entlastung der Eltern von Patienten mit Spinaler Muskelatrophie

Thorsten Langer', Jana Willems'2, Erik Farin-Glattacker ${ }^{2}$

'Universitätsklinikum Freiburg, Zentrum für Kinder- und Jugendmedizin, Klinik für Neuropädiatrie und Muskelerkrankungen, Freiburg, Deutschland; ${ }^{2}$ Universitätsklinikum Freiburg, Sektion für Rehabilitationsforschung und Versorgungsforschung, Freiburg, Deutschland

Die Spinale Muskelatrophie Typ 1 ist eine schwer verlaufende, seltene, neuromuskuläre Erkrankung mit komplexer Versorgungssituation, die durch folgende Merkmale gekennzeichnet ist:

- Krankheitsverlauf und Versorgungsbedarf mit hoher Dynamik,

- multiprofessionelle Versorgung

- große räumliche Distanzen zwischen lokalen Behandlern und Muskelzentren

- hoher Beratungsbedarf der Familien.[1]

Häufig ist die Versorgung zwischen den Behandlern und Versorgungssektoren fragmentiert und unzureichend aufeinander abgestimmt bzw. integriert. Eine unzureichende Behandlungsintegration kann die Versorgungsqualität beeinträchtigen und zu chronischen Belastungen für Eltern führen.

In einem 3-jährigen Projekt wird zunächst ein Patient-Reported-Outcome-Instrument zur Qualität der Behandlungsintegration [2] bei chronischen Erkrankungen in der Pädiatrie für den deutsch-sprachigen Raum validiert. Nachfolgend werden in einer IST-Analyse die Erfahrungen von Eltern und niedergelassenen Kinder- und Jugendärzten analysiert, die an der Versorgung von Kindern mit SMA beteiligt sind. Dabei kommen leitfaden-gestützte Interviews sowie Versorgungstagebücher zum Einsatz. Darauf aufbauend wird ein spezifisches Case-Management konzeptionalisiert und durch eine digitale Plattform ergänzt.

Im Rahmen einer quasi-experimentellen Studie wird die Auswirkung der Intervention im Vergleich zur einer Vergleichsgruppe mit einem mixed methods-Ansatz untersucht $(n=2 \times 25)$. Als primäre Endpunkte werden die gesundheitsbezogene Lebensqualität der Eltern und sowie die Qualität der Behandlungsintegration (PICS) untersucht. Sekundäre Endpunkte sind die Inanspruchnahme medizinischer Leistungen (Patient und Eltern), die Kosten sowie Daten zur Prozessqualität.

Wichtiges Element des Forschungsprozesses ist die Beteiligung von Patientenvertretern und Betroffenenverbänden (z. B. Deutsche Gesellschaft für Muskelkranke (DGM) und Kindernetzwerk e. V.) im Rahmen von regelmässig stattfinden Symposien. Dieser Beteiligungsprozess wird im Rahmen einer Begleitforschung evaluiert.[3]

Am Ende des Projekts liegt nach der Validierungsstudie des PICS-Fragebogens erstmals ein deutschsprachiges Instrument vor, mit dem die Qualität der Behandlungsintegration bei chronischen Erkrankungen gemessen werden kann. Es wird für andere Forschungsprojekte und in Qualiätsverbesserungsmaßnahmen eingesetzt werden können. Nach der Evaluation des Case-Managements liegen Ergebnisse zu Machbarkeit, erwartbaren Effektgrößen und Prozessqualität vor. Auf dieser Grundlage können zukünftige cluster-randomisierte Studien geplant werden. Es ist zu erwarten, dass die Intervention auf andere Zentren, die Patienten mit SMA und anderen seltenen Erkrankungen betreuen, übertragbar sein wird.

\footnotetext{
Literatur

1. Mercuri E et al (2018) Diagnosis and management of spinal muscular atrophy: Part 1:Recommendations for diagnosis, rehabilitation,orthopedic and nutritional care.

Neuromuscul Disord 28:103-115

2. Ziniel $\mathrm{SI}$, Rosenberg HN, Bach AM, et al. Val
}

DGSPJ-PO 39

KOMKEK - Entwicklung einer Fortbildung für Behandelnde von Krebserkrankten zur Stärkung der Kompetenz im Umgang mit krebskranken Eltern minderjähriger Kinder

Corinna Bergelt, Lene Marie Johannsen, Wiebke Frerichs, Laura Inhestern

Universitätsklinikum Hamburg-Eppendorf, Institut und Poliklinik für

Medizinische Psychologie, Hamburg, Deutschland

Hintergrund: Psychoonkologische Angebote für Erkrankte und Angehörige sind integrale Bestandteile einer modernen patientenorientierten onkologischen Versorgung. Die psychoonkologische Versorgung soll und kann aber in der Regel nur für hochbelastete Patientinnen und Patienten von Spezialisten durchgeführt werden, daher ist eine Basis-Qualifizierung aller an der Behandlung beteiligten Berufsgruppen auch für psychosoziale Themen erforderlich.

Krebserkrankte, die minderjährige Kinder haben, durchleben spezifische Belastungsaspekte und Unterstützungsbedürfnisse. Jedoch äußert nur ein Bruchteil betroffener Eltern von sich aus den Wunsch nach Unterstützung für sich als Elternteil oder für die Kinder. Gleichzeitig werden psychosoziale Themen selten von (onkologischem) Fachpersonal eigeninitiativ angesprochen, u. a. weil sich diese in der Kommunikation unsicher fühlen. Um mögliche Belastungen betroffener Eltern besser adressieren sowie diese bei Bedarf an familienorientierte Beratungsstellen weiterzuleiten zu können, ist eine Stärkung der kommunikativen Kompetenz und die Sensibilisierung der Behandelnden für elternbezogener Themen dringend erforderlich.

Ziele: Das Projekt KOMKEK zielt auf die Verbesserung der Versorgung krebskranker Eltern und ihrer Kinder durch die Sensibilisierung behandelnder Personen ab. Durch ein Präsenztraining, bei dem die Teilnehmenden anwesend sind, und eine Online-Schulung soll medizinisches und psychosoziales Personal in seinen fachlichen Kompetenzen gestärkt werden.

Methodik: Basierend auf einer umfassenden Literaturrecherche und qualitativen Interviews mit Behandlern, betroffenen Eltern und Expertinnen und Experten zum Thema Kinder krebskranker Eltern, werden in der ersten Projektphase das Präsenztraining und die Online-Schulung entwickelt. In der zweiten Projektphase wird eine Pilotstudie zur Evaluation des Trainings mithilfe einer randomisiert-kontrollierten Studie mit dreiarmiger Randomisierung (Präsenztraining vs. Online-Schulung vs. Wartekontrollgruppe) durchgeführt.

Zwischenergebnisse: Aktuell wurden die inhaltlichen Vorarbeiten (systematische Literaturübersicht \& Interviews mit Behandelnden, betroffenen Eltern und Expertinnen und Experten) abgeschlossen, das Evaluationskonzept der Studie entwickelt und die Inhalte der Präsenz- sowie OnlineFortbildung entworfen. Ein erster Ergebnisausschnitt wird auf dem Kongress vorgestellt.

Ausblick: Nach Abschluss des Projekts liegt erstmalig für den deutschen Sprachraum eine systematische Intervention für Behandelnde zum Thema „Kinder krebskranker Eltern“ vor. Durch die Fortbildung kann die Kompetenz und Kommunikation der Behandelnden im Umgang mit betroffenen Eltern und Kindern gestärkt werden, damit betroffene Familien emotionale Entlastung erfahren und bei Bedarf an spezialisierte kind- oder familienzentrierte Angebote weitervermittelt werden können.

\section{DGSPJ-PO 40}

Anwendung von Arznei- und Nahrungsergänzungsmitteln im Kindes- und Jugendalter in Deutschland - Ergebnisse der KIGGSStudie

Hiltraud Knopf', Giselle Sarganas², Daniel Grams², Yong Du², Christina Poethko-Müller ${ }^{2}$

'ehemals Robert Koch-Institut, Abteilung für Epidemiologie und Gesundheitsberichterstattung, Berlin, Deutschland; ${ }^{2}$ Robert Koch-Institut, Abteilung für Epidemiologie und Gesundheitsberichterstattung, Berlin, Deutschland 
Mit den Ergebnissen der Basiserhebung des Kinder- und Jugendgesundheitssurveys („KiGGS-Basis“) standen in Deutschland erstmals bundesweit bevölkerungsbezogene Indikatoren der aktuellen Anwendung von Arznei- und Nahrungsergänzungsmitteln (NEM) für Kinder und Jugendliche zur Verfügung. Mit der erneuten Untersuchung und Befragung in KiGGS Welle 2 liegen aktuelle Ergebnisse vor, die es erlauben, zeitliche Veränderungen im Anwendungsverhalten abzubilden.

Aus KiGGS Welle 2 (2014-2017) liegen für 3462 drei- bis 17 Jährige Daten aus einem standardisierten Interview zur aktuellen Anwendung (in den letzten 7 Tagen vor Untersuchung) von Arzneimitteln (AM) und NEM vor. Für den zeitlichen Vergleich wurden die Daten von 3- bis 17-Jährigen aus KiGGS-Basis $(n=14.870)$ genutzt.

Die Prävalenz der Anwendung von mindestens einem AM oder NEM betrug in KiGGS Welle $236,4 \%$ (95\% KI 34,1-38,8 \%). Bei den 14- bis 17-Jährigen war die Prävalenz mit 46,5 \% am höchsten und bei Mädchen (M) signifikant höher als bei Jungen (J) (56,4 \% vs. 37,3 \%). Nur bei Mädchen zeigten sich neben dem Alter signifikante Unterschiede nach Migrationshintergrund. Am häufigsten wurden Präparate zur Behandlung des Respirationstraktes (ATC-Code R: M: 14,2 \%; J: 14,9\%) angewendet. Es folgten Varia (ATC-Code V: M: 8,7 \%; J: 9,3 \%) und Muskel-Skelettsystem (ATC-Code M: M: 8,9 \%, J: 5,8 \%). Im Vergleich zur Basiserhebung zeigte sich ein signifikanter Rückgang in der Anwendungsprävalenz. Dieser Rückgang war vor allem durch niedrigere Anwendungsprävalenzen in den ATC-Klassen „N Nervensystem“, „J Antiinfektiva zur systemischen Anwendung “ und „H Systemische Hormonpräparate, excl. Sexualhormone und Insuline" bedingt und bezog sich in erster Linie auf die Verordnungsmedikation.

Die dargestellten Ergebnisse beschreiben Eckpunkte des Gebrauchs von AM und NEM unter Einschluss der Selbstmedikation für Kinder in Deutschland. Sie können damit das Anwendungsverhalten abbilden und ergänzen die Informationen aus Verordnungsdaten der GKV.

\section{Chronische Erkrankungen 2}

\section{DGSPJ-P0 41 \\ Psycho-soziale Belastungen von Kindern und Jugendlichen mit Typ 1 Diabetes und ihren Familien}

\section{Andrea Dehn-Hindenberg, Karin Lange}

Medizinische Hochschule Hannover, Medizinische Psychologie, Hannover, Deutschland

Fragestellung: Eine chronische Erkrankung stellt Kinder und Jugendliche vor große Herausforderungen. Sie sind körperlich und psychisch stärkeren Belastungen ausgesetzt als Gleichaltrige ohne eine chronische Krankheit. Dies gilt ebenso für die Eltern und Geschwister. Deshalb richtet sich der Fokus der Langzeittherapie nicht allein auf das betroffene Kind, sondern bezieht das gesamte Familiensystem mit ein. Beispielsweise ist bei Typ 1 Diabetes die Qualität der Stoffwechseleinstellung zu sichern, indem das Familiensystem und das Gesundheits- und Selbstmanagement der betroffenen Kinder und Jugendlichen gestärkt werden. Um effektive und alltagsorientierte Hilfen anbieten zu können, sind belastbare Erkenntnisse darüber notwendig, welche Hilfsleistungen die Familien bislang nutzen. Die Studie gibt Aufschluss über die Fragen der aktuellen Situation und den psycho-sozialen Unterstützungsbedarf von Kindern und Jugendlichen mit Typ 1 Diabetes und ihren Familien.

Methodik: In 9 Diabeteszentren wurden bundesweit Eltern von Kindern mit einer Diabetesmanifestation vor dem 14. Lebensjahr mittels eines strukturierten Fragebogens zu den psycho-sozialen Folgen der Stoffwechselstörung des Kindes, zur psycho-sozialen Belastung und zum Unterstützungsbedarf aller Familienmitglieder anonym befragt. (Messinstrumente: CRF (Soziodemografische Daten der Eltern und zentrale Diabetesdaten des Kindes), Fragebogen zu beruflichen Folgen der Diabetesmanifestation der Eltern, zur Inanspruchnahme von Versorgunsgleistungen und Hilfsangeboten, und zur psychosozialen Belastung aller Familienmitglieder sowie ein semistrukturierter Fragebogen zum Unterstützungsbedarf). Ergebnisse: Es beteiligten sich 1144 Eltern an der Studie (Rücklauf 81 \%). Nach ihren Angaben haben 48,3 \% der Kinder und Jugendlichen mit Typ 1 Diabetes eine hohe Alltagsbelastung, 22,9 \% haben eine hohe körperliche Belastung. Bei $47 \%$ der betroffenen Kinder ist die psychische Belastung hoch. Die Eltern sind durch die Diabeteserkrankung des Kindes ebenfalls hoch belastet. So ist der Belastungsrad im Alltag bei 52,6\% der Mütter und 30,6 \% der Väter hoch. Eine hohe psychische Belastung haben $62 \%$ der Mütter und $41 \%$ der Väter. Bei $9 \%$ der Kinder und Jugendlichen mit Typ-1-Diabetes und bei $13 \%$ der Mütter wurde eine psychische Erkrankung diagnostiziert. (Bei alleinerziehenden Müttern liegt der Anteil an psychischen Erkrankungen bei $18 \%$ ). Einen hohen Unterstützungsbedarf haben $46,6 \%$ der Familien.

Diskussion und Schlussfolgerungen: Die Studie belegt die psychosozialen Folgen der Diabetesdiagnose für Kinder und Jugendliche und ihre Familien und deren hohen Unterstützungsbedarf bei der Krankheitsbewältigung und bei der Integration der Therapie in den Alltag. Es stellt sich die Frage wie die psycho-soziale Familiensituation bei der Therapie berücksichtigt und welche frühzeitigen Hilfen angeboten werden sollten, um Folgeerkrankungen der Kinder und Jugendlichen mit Typ 1 Diabetes und ihren Familien zu verhindern bzw. zu reduzieren. 


\section{DGSPJ-PO 42}

\section{Sozialstatus, Diabetesoutcomes und Diabetesversorgung bei Kindern und Jugendlichen mit Typ-1 Diabetes in Deutschland: Die Ergebnisse der DIAS-Studie (Diabetes and Social Disparities)}

Esther Müller-Godeffroy', Kirsten Mönkemöller', Eggert Lilienthaß’, Bettina Heidtmann ${ }^{4}$, Marinne Becker ${ }^{5}$, Lutz Feldhahn ${ }^{6}$, Markus Freff', Dörte Hilgard ${ }^{8}$, Beate Krone, Matthias Papsch ${ }^{10}$, Andrea Schumacher ${ }^{11}$, Karl Otfried Schwab ${ }^{12}$, Heidemarie Schweiger ${ }^{13}$, Johannes Wolf ${ }^{14}$, Reinhard Holl ${ }^{15}$, Esther Bollow ${ }^{15}$

'Universitätsklinikum Schleswig-Holstein, Campus Lübeck, Klinik für Kinder- und Jugendmedizin, Lübeck, Deutschland; ${ }^{2}$ Kinderkrankenhaus Amsterdamer Straße, Köln, Klinik für Kinder- und Jugendmedizin, Köln, Deutschland; ${ }^{3}$ St. Josef-Hospital, Ruhr-Universität Bochum, Klinik für Kinder- und Jugendmedizin, Bochum; ${ }^{4}$ Katholisches Kinderkrankenhaus Wilhelmstift, Kinderdiabetologie, Hamburg, Deutschland; ${ }^{5} \mathrm{HELIOS}$ Dr. Horst Schmidt Kliniken Wiesbaden, Klinik für Kinder- und Jugendmedizin, Wiesbaden, Deutschland; ${ }^{6}$ Klinikverbund-Suedwest, Klinik für Kinder- und Jugendmedizin, Böblingen, Deutschland; ${ }^{7}$ Darmstädter Kinderkliniken Prinzessin Margaret, Klinik für Kinder- und Jugendmedizin, Darmstadt; ${ }^{8}$ Gemeinschaftskrankenhaus Herdecke, Kinderdiabetologie, Herdecke; ${ }^{9}$ Klinikum Bremen-Nord, Kinderklinik, Bremen; ${ }^{10}$ Marienhospital Gelsenkirchen, Klinik für Neonatologie, Kinder- und Jugendmedizin, Gelsenkirchen; ${ }^{11}$ Klinik am Eichert, Zentrum für Kinder- und Jugendmedizin, Göppingen; ${ }^{12}$ Universitätskinikum Freiburg, Klinik für Kinder- und Jugendmedizin, Freiburg; ${ }^{13} \mathrm{Klinikum}$ Fürth, Klinik für Kinder und Jugendliche, Fürth; ${ }^{14} \mathrm{St}$. Vincenz-Krankenhaus Paderborn, Kinderklinik, Paderborn; ${ }^{15}$ Universität Ulm, Institut für Epidemiologie und medizinische Biometrie, ZIBMT, Ulm, Deutschland

Fragestellung: Es wurde die Assoziation zwischen Sozialstatus (socioeconomic status, SES) und diabetesbezogenen Outcomes bei Kindern mit Typ-1 Diabetes in Deutschland untersucht.

Material und Methode: 1829 Kinder und Jugendliche mit Typ-1 Diabetes mellitus unter 18 Jahren aus 13 deutschen Diabeteszentren wurden zwischen Juni 2013 und Juni 2014 in die DIAS-Studie eingeschlossen. Die Daten wurden im Rahmen des DPV-Registers (Diabetes-Patienten-Verlaufsdokumentation) erfasst. Der Sozialstatus wurde in Anlehnung an den Winkler-Index gemessen, der auf Angaben zum schulischen und beruflichen Bildungsniveau, zur beruflichen Stellung und zur Einkommenssituation basiert. Die Assoziationen zwischen Sozialstatus und den Diabetesoutcomes wurden mittels multivariater Regressionsmodelle analysiert, wobei für Alter, Geschlecht, Diabetesdauer und Migrationsstaus adjustiert wurde.

Ergebnisse: Ein niedriger SES war mit schlechteren diabetesbezogenen Outcomes assoziiert: mit höherem HbAlc $(64,4 \mathrm{mmol} / \mathrm{mol})$, weniger häufiger Nutzung von Insulinpumpentherapie (42,9\%), weniger täglichen Blutzuckerselbstmessungen (self-monitoring of blood glucose, SMBG) $(5,7)$, häufigeren Krankenhaustagen pro Patientenjahr $(5,8)$ im Vergleich zu mittlerem/höherem SES (HbAlc: $61,3 \mathrm{mmol} / \mathrm{mol}$, $p<0,001 / 59,8 \mathrm{mmol} / \mathrm{mol}, p<0,0001$; Anteil an Pumpentherapie: $54,5 \%$, $p<0,01 / 54,9 \%, p<0,01$; SMBG: $6,0, p<0,01 / 6,1, p<0,01$; Krankenhaustage: $4,5, p<0,0001 / 3,4, p<0,0001)$. Die Berücksichtigung des Migrationshintergrundes hatte dabei nur einen geringen Einfluss auf die Ergebnisse. Diskussion: Ein niedriger Sozialstatus ist bei Kindern mit Diabetes Typ 1 auch in Deutschland mit ungünstiger Stoffwechsellage, geringerem $\mathrm{Zu}$ gang zu fortgeschrittenen Therapien und häufigerer Inanspruchnahme stationärer Versorgung assoziiert. Ähnliches gilt vermutlich auch für andere chronische Erkrankungen im Kindesalter. Eine routinemäßige Erhebung des SES durch geeignete Indikatoren in den Gesundheitsregistern erscheint wünschenswert, da sie für die Versorgungsforschung und Qualitätssicherung wichtigen Daten liefern und zu einer besseren Gestaltung der Versorgung von Risikogruppen wie beitragen kann.

Schlussfolgerung: Der Sozialstatus ist neben anderen Sozialvariablen wie Migrationshintergrund und Familienstand ein bedeutsamer Prädiktor für Gesundheitsoutcomes, der in der Qualitätssicherung, der Gesundheitsstatistik und Versorgungsforschung mehr Berücksichtigung erfahren sollte.
Die Ergebnisse wurden publiziert in Pediatr Diabetes. 2019;1-8. https:// doi.org/10.1111/pedi.12847.

\section{DGSPJ-PO 43 \\ PART-CHILD: Evaluation einer ICF-CY-basierten komplexen Versorgungsmaßnahme zur Verbesserung der partizipativen Entscheidungsfindung an Sozialpädiatrischen Zentren: Studiendesign einer cluster-randomisierten Studie im Stepped- Wedge-Design.}

Freia De Bock', Tatiana Görig', Sabine Georg', Heike Philippi', Dorle Hoffmann ${ }^{3}$, Jochem König ${ }^{3}$, Michael Siegfried Urschitz ${ }^{3}$, Michael Eichinger ${ }^{1}$ Medizinische Fakultät Mannheim, Universität Heidelberg, Mannheimer Institut für Public Health, Sozial- und Präventivmedizin, Mannheim, Detschland; ${ }^{2}$ Sozialpädiatrisches Zentrum Frankfurt Mitte, Frankfurt am Main, Deutschland; ${ }^{3}$ Universitätsmedizin Mainz, Institut für medizinische Biometrie, Epidemiologie und Informatik, Mainz, Deutschland

Hintergrund: Um die Versorgungsqualität für Kinder und Jugendliche mit chronischen Krankheiten oder Behinderungen zu verbessern, hat die WHO die International Classification of Functioning, Disability and Health for Children and Youth (ICF-CY) publiziert, die die Domänen Aktivitäten, Partizipation und Kontextfaktoren in den Mittelpunkt rückt. Gemeinsam mit einer stärkeren Teilhabeorientierung der Versorgung und einer Stärkung der partizipativen Entscheidungsfindung könnte die ICF-CY einen wichtigen Beitrag zur Verbesserung der Versorgungsqualität leisten. Eine Implementierung der ICF-CY im Versorgungsalltag wurde bisher jedoch nicht konsequent versucht.

Das Ziel des Projekts PART-CHILD ist es, die Implementierung einer komplexen ICF-CY-basierten Versorgungsmaßnahme an Sozialpädiatrischen Zentren (SPZ) zu evaluieren. Die Versorgungsmaßnahme umfasst (1) eine dreimodulare Mitarbeiterschulung zur ICF-CY und zur teilhabe- und patientenzentrierten Gesprächsführung, (2) die Bereitstellung der Planungs- und Dokumentationssoftware ICF-Add-In, die die Anwendung der ICF-CY im SPZ-Alltag unterstützt, sowie (3) eine mehrmonatige interdisziplinäre Begleitung des Transfers von Schulungsinhalten in den SPZAlltag (z. B. Supervision).

Fragestellung: Erhöht sich durch die Implementierung der dreimodularen ICF-CY-basierten Versorgungsmaßnahme für Kinder und Eltern (1) der Grad an partizipativer Entscheidungsfindung, (2) ihre Zufriedenheit mit der Versorgung, (3) ihre Lebensqualität sowie (4) die Teilhabe der Patienten im Alltag?

Methoden: Die Effektevaluation an $15 \mathrm{SPZ}$ erfolgt durch eine cluster-randomisierte Studie im Stepped-Wedge-Design zwischen November 2018 und Juli 2020. Insgesamt werden ca. 3000 Kinder und 12.000 Eltern eingeschlossen. Primärer Endpunkt ist die von den Eltern empfundene partizipative Entscheidungsfindung. Sekundäre Endpunkte sind die partizipative Entscheidungsfindung aus Sicht der Kinder (falls $>7$ Jahre), die Zufriedenheit mit der Versorgung, die gesundheitsbezogene Lebensqualität sowie die Teilhabe der Kinder. Die Analyse erfolgt mit hierarchischen Modellen. Im Rahmen der Prozessevaluation werden quantitative (u. a. Fachkraftsurvey) und qualitative Daten (u. a. semistrukturierte Interviews mit Fachkräften) analysiert.

Ergebnisse: Im Rahmen des Kongresses werden Details zur (1) Versorgungsmaßnahme und zum (2) Studiendesign der Effekt- und Prozessevaluation vorgestellt.

Schlussfolgerungen: Durch die Stärkung einer teilhabeorientierten Versorgung hat die ICF-CY-basierte Versorgungsmaßnahme das Potenzial, die Versorgungsqualität für Kinder mit chronischen Krankheiten zu verbessern. Bei nachgewiesener Effektivität der Versorgungsmaßnahme wäre auf Grund des modularen Aufbaus eine Ausweitung des Programms auf weitere SPZ und die Adaptierung für andere pädiatrische Versorgungssysteme (z. B. Hochschulambulanzen) zeitnah möglich. 


\title{
Abstracts der 57. Herbsttagung der Deutschen Gesellschaft für Kinderchirurgie (DGKCH)
}

\author{
Wissenschaftliche Leitung \\ Prof. Dr. Stephan Kellnar
}

\section{Freie Vorträge}

\section{Das chirurgisch kranke Frühgeborene}

\section{DGKCH-FV 01}

Leistenhernie beim Frühgeborenen $<1500 \mathrm{~g}$. Wann ist der beste Zeitpunkt für die operative Korrektur?

Vincent Hoffmann', Katharina Karsten², Stefanie Märzheuser

${ }^{1}$ Charité - Universitätsmedizin Berlin, Campus Virchow, Kinderchirurgie, Berlin, Deutschland; ${ }^{2}$ Charité - Universitätsmedizin Berlin, Campus Virchow, Neonatologie, Berlin

Der beste Zeitpunkt für eine operative Korrektur der Leistenhernie bei Frühgeborenen wird in der Literatur kontrovers diskutiert - hier gilt es, die Risiken einer frühen Operation (postoperative Nachbeatmung, Infektion und Sepsis, intraoperative Bradykardien und anspruchsvoller Situs) gegenüber dem Inkarzerationsrisiko der Hernie bei späterer Operation abzuwägen.

Methoden: In einer retrospektiven Studie wurde der Operationszeitpunkt der Leistenhernien von 66 Frühgeborenen mit einem Geburtsgewicht von $<1500 \mathrm{~g}$ in einem Patientenkollektiv der der Charité Universitätsmedizin Berlin, die zwischen dem 01.01.2010 und dem 31.10.2016 operiert wurden, untersucht.

Als früher Zeitpunkt der operativen Korrektur wurde die Operation während des ersten stationären Aufenthalts definiert. Kinder, die spät operiert wurden, verließen das Krankenhaus zunächst und wurden zur Operation elektiv erneut aufgenommen.

Für die Auswertung wurde eine Unterteilung in zwei Gruppen vorgenommen. Eine Gruppe umfasste Patienten ohne perioperative Komplikation (Gruppe A, $n=69$ ), eine weitere Gruppe bestand aus Patienten mit perioperativer Komplikation (Gruppe B, $n=17$ ).

Die Unterschiede bei den Komorbiditäten, dem Geburtsgewicht, dem Gestationsalter oder dem CRIB-Score wurden analysiert. Der Operationszeitpunkt wurde verglichen, außerdem wurde ein Zusammenhang mit perioperativen Komplikationen geprüft.

Ergebnisse: Es wurden 86 Operationen bei 66 Patienten mit einem Geburtsgewicht von $<1500 \mathrm{~g}$ und einem Leistenbruch analysiert. 19,8 \% der Frühgeborenen erlitten während oder nach der Hernienoperation Komplikation wie Wundinfektion, stimulationspflichtige Bradykardien, postoperative Nachbeatmung, Bluttransfusion, Hernienrezidiv und Sauerstoffsättigungsabfall.

Beide Gruppen waren im Hinblick auf ihre Vorerkrankungen gleich verteilt. Frühchen in der Komplikationsgruppe hatten ein signifikant geringeres Gestationsalter, ein geringeres Geburtsgewicht und wurden früher operiert. Diese Aspekte stellten damit in unserer Arbeit die größten Risikofaktoren für eine Komplikation bei der Leistenhernienoperation dar. Kinder, bei denen eine Komplikation auftrat, wurden zu $95 \%$ früh operiert, von den spät operierten Kindern hingegen erlitten nur $6 \%$ eine Komplikation. Aus diesen Daten berechneten wir ein relatives Risiko. Kinder, die früher operiert wurden, hatten ein 7,3-faches, signifikant höheres Risiko, eine perioperative Komplikation zu entwickeln, als die Kinder, die zu ei- nem späteren Zeitpunkt elektiv operiert wurden. Das Risiko einer Inkarzeration lag im gesamten Kollektiv bei 3,5 \% der Patienten Schlussfolgerung: In unserer Untersuchung waren die größten Risikofaktoren einer perioperativen Komplikation ein geringes Geburtsgewicht, ein geringes Gestationsalter und ein früher Operationszeitpunkt. Damit liefert diese Arbeit weitere Evidenz für einen späten Operationszeitpunkt, da das Inkarzerationsrisiko unseres Erachtens nicht die Risiken einer frühen Operation aufwiegt.

\section{DGKCH-FV 02}

Ein Frühgeborenes mit Ösophagusatresie Vogt 3b, kompliziert durch großen Trachealwanddefekt, Fallot'scher Tetralogie mit rechts deszendierender Aorta, A. lusoria sowie einer kloakalen Fehlbildung: Eine Herausforderung an die Akut- und Folgetherapie

Peter Knorr, Tobias Schuster

Klinikum UNI Augsburg, Kinderchirurgie, Augsburg, Deutschland

Wir berichten über ein weibliches Frühgeborenes (31. SSW, 968 g. GG) mit einer komplexen VACTERL-Assoziation. Die akut im Vordergrund stehende Ösophagusatresie (Vogt 3b) verkomplizierte sich durch ein komplexes Herzvitium mit Fallot'scher Tetralogie in Kombination mit einer rechts deszendierenden Aorta sowie einer A. lusoria. Präoperativ nicht bekannt war ein ausgedehnter Trachealwanddefekt um die Carina. Des weiteren zeigt die Patientin eine kloakale Malformation mit Vaginal- und Uterusatresie sowie Nierenagenesie einseitig. Der common channel ist mit $1-1,5 \mathrm{~cm}$ kurz.

Die operative Planung der Akuttherapie beinhaltete nun zunächst eine linksseitige extrapleurale Thoracotomie mit Fistelverschluß und End zu End-Anastomose des Ösophagus sowie Anlage A. p.. Der nun intraoperativ sichtbare congenitale Trachealwanddefekt mit Trachealeröffung zwang uns akut zur Änderung des Vorgehens mit nun Verschluß der tracheoösophagealen Fistel, Versuch einer Übernähung der Trachea und Anlage eines Gastrostomas. Ein luftdichter Trachealverschluß war, unter andrem Aufgrund der kreuzenden A. lusoria, nicht möglich, Zusatzmaßnahme war eine Verklebung mit Vicrylnetz und Fibrinkleber sowie Einlage einer extrapleuralen Saugdrainage. Die Trachea war nach etwa 7 Tagen schlagartig dicht. Etwa 3 Wochen später erfolgte zunächst eine Tracheoskopie, im Anschluß die unkomplizierte direkte End-zu-End-Anastomose des Ösophagus bewußt über einen transpleuralen Zugang, um den Trachealdefekt nicht erneut zu tangieren. Im weiteren Verlauf mußte die Ösophagusanastomose bis dato $2 \times$ dilatiert werden.

Mittlerweile ist die Patientin oral komplett ernährbar und hat ihr Geburtsgewicht verdreifacht. Die Entlassung steht bevor. In der weiteren Planung nun die operative Therapie der kloakalen Fehlbildung. 


\section{Chirurgie im Jugendalter}

\section{DGKCH-FV 03}

Evaluation of our paradigm shift and its outcome: conservative treatment of pediatric (second degree) burns between 2002-2016

Katharina Schriek', Mechthild Sinnig', Barbara Ludwikowski'

${ }^{1}$ Kinder- und Jugendkrankenhaus Auf der Bult, Zentrum für Schwerbrandverletzte Kinder, Hannover, Germany; ${ }^{2}$ Kinder- und Jugendkrankenhaus Auf der Bult, Kinderchiurgie und -urologie, Hannover, Germany

Purpose: The different options of conservative treatments in second degree burns have been widely discussed through out the last 15 years. We present our data of second degree burns in children starting 2002 and evaluated the effectiveness of our paradigm shift in the treatment of partial thickness burns in children over the period of the last 13 years: Starting with the first treated cases in our pediatric burn unit 2004 up to the use of a resorbable skin substitute as the goldstandard since 2010 .

A retrospective study (2002-2016) was conducted to evaluate the use of in respect of burn depth, total body surface area (TBSA), number of dressing changes, need for skin grafting and length of hospital stay.

Results: 2134 children have been treated in our institution between $2002-$ 2016. 1735 children had a second degree burn $\left(2 \mathrm{a}^{\circ}=908,2 \mathrm{~b}^{\circ}=1081 \mathrm{pa}-\right.$ tients) and 324 patients had a third degree burn. 1063 patients were treated with Suprathel ${ }^{\circ}$. The need for split thickness skin transplantation in second degree burns dropped inversely proportional to the use of Suprathel ${ }^{\circ}$. and was reduced by $74 \%$ during the last 14 years. The number of dressing changes under general anaesthesia had decreased by more than $40 \%$. Conclusions: The use of Suprathel ${ }^{\circledR}$ provides advantages regarding the need for split thickness skin transplantation, number of dressing changes and length of hospital stay.

\section{Diagnostik und Therapie der komplizierten Appendizits}

\section{DGKCH-FV 04}

Methoden der künstlichen Intelligenz bei der akuten Appendizitis im Kindesalter: ein untersucherunabhängiger Ansatz

Marc Reismann', Alessandro Romualdi', Jim Kallarackal', Josephine Reismann' ${ }^{1}$ Maximiliane Minderjahn' ${ }^{1}$, Natalie Kiss' ${ }^{1}$, Martina Schad ${ }^{4}$

${ }^{1}$ Charite Kliniken f. Kinderheilkunde und Kinderchirugie, Kinderchirurgie, Berlin, Deutschland; ${ }^{2}$ OakLabs GmbH, Computational Science, Hennigsdorf; ${ }^{3}$ OakLabs GmbH, Physics, Hennigsdorf,; ${ }^{4}$ OakLabs GmbH, Biology, Hennigsdorf

Hintergrund: Die Unterscheidung von unkomplizierter (histopathologisch phlegmonöser) und komplizierter (gangränöser/perforierender) Appendizitis ist insbesondere hinsichtlich konservativer Therapiestrategien bei der unkomplizierten Form von Bedeutung. Während der Wert der klinischen Untersuchung durch eine unzureichende Interobserver-Zuverlässigkeit limitiert ist, lassen Methoden der künstlichen Intelligenz (KI) möglicherweise eine optimale Nutzung objektiver Parameter zur Differenzierung zu.

Fragestellung: Lassen sich KI-Methoden auf Basis objektiver Parameter zur Diagnostik bei der Appendizitis im Kindesalter und insbesondere zur Differenzierung von unkomplizierter und komplizierter Appendizitis nutzen?

Material und Methoden: Differentialblutbilder, C-reaktives Protein (CRP) und sonographisch gemessener Appendixdurchmesser von 937 Patienten im Alter von 0-17 Jahren mit histopathologisch nachgewiesener Appendizitis und von 240 Patienten mit histopathologisch negativem Befund wurden retrospektiv mit Algorithmen aus dem maschinellen Lernen (ML) und der KI analysiert. Es resultierten differenzierte Biomarker-Signaturen für die Diagnose der akuten Appendizitis und der komplizierten Entzündung. Ergebnisse: Die Accuracy der Biomarker-Signatur für die Diagnose einer Appendizitis betrug 90\% (93\% Sensitivität, 67\% Spezifität), während die Accuracy für die korrekte Erkennung einer komplizierten Entzündung $70 \%$ betrug (76\% Sensitivität, $67 \%$ Spezifität). Unsere BiomarkerSignaturen übertrafen damit die Vorhersagewerte für die einzelnen Laborwerte wie CRP, Leukozyten- und Neutrophilenzahlen.

Schlussfolgerung: Die vorgestellte Methode hat das Potenzial, die diagnostischen Möglichkeiten bei der akuten Appendizitis zu erweitern, und demonstriert die Fähigkeit von Algorithmen aus KI und ML, die Diagnose auf der Grundlage objektiver diagnostischer Parameter erheblich zu verbessern.

\section{DGKCH-FV 05}

Der Wert von Labor- und sonographischer Diagnostik bei der Differenzierung von unkomplizierter und komplizierter Appendizitis

Josephine Reismann', Tanja Rawolle', Maximiliane Minderjahn', Natalie Kiss', Karin Rothe ${ }^{1}$, Marc Reismann ${ }^{2}$

'Charité - Universitätsmedizin Berlin, Kinderchirurgie, Berlin, Deutschland; ${ }^{2}$ Charite Kliniken $\mathrm{f}$. Kinderheilkunde und Kinderchirugie, Kinderchirurgie, Berlin, Deutschland

Hintergrund: Immunologische und epidemiologische Studien weisen darauf hin, dass die klinisch unkomplizierte (histologisch phlegmonös) und die komplizierte Appendizitis (histologisch gangränös/perforierend) unabhängige Entitäten auf der Basis unterschiedlicher Pathophysiologien darstellen. Dies ist insbesondere für konservative Therapiestrategien für die unkomplizierte Form von Bedeutung.

Fragestellung: Welche Parameter im Rahmen der Labor- und sonographischer Routinediagnostik können für die Unterscheidung von unkomplizierter und komplizierter Appendizitis genutzt werden?

Material und Methoden: Die Daten aller Patienten im Alter von 0 bis 17 Jahren, die von Dezember 2006 bis September 2016 appendektomiert 
worden waren, wurden hinsichtlich epidemiologischer Parameter, der zeitlichen Verläufe (Symptombeginn bis Blutentnahme), der histopathologischen Beurteilung, der Differentialblutbilder, der CRP-Werte und definierter sonographischer Parameter ausgewertet. Das Signifikanzniveau wurde bei $p>0.01$ festgelegt.

Ergebnisse: Es konnten 1041 Patienten in die Studie eingeschlossen werden. Die Auswertung der Laborwerte über die Zeit ergab insbesondere für CRP und Leukozyten ein signifikant differenziertes Bild. Die phlegmonöse Entzündung war durch eine zeitlich stabile Eosinophilie gekennzeichnet $(p<0.01)$. Eine Analyse mittels Receiver-Operating-Characteristic-Kurve zeigte in Bezug auf alle Laborparameter eine grundsätzliche Unterscheidbarkeit; klinisch sinnvolle Cut-off-Werte ließen sich jedoch nicht festlegen. Sonographisch zeigte der Appendixdurchmesser von $>6 \mathrm{~mm}$ eine Sensitivität von $98 \%$ für die Differenzierung der komplizierten Appendizitis. Das Vorhandensein eines Appendicolithen war mit einer Spezifität von 98\% assoziiert. Hinweisgebend war insbesondere die Einschätzung des Kinderradiologen, ob eine Perforation vorlag $(p<0,001)$.

Schlussfolgerung: In der Analyse der Laborparameter lassen sich deutliche Hinweise darauf finden, dass unkomplizierte und komplizierte Appendizitis unabhängige pathophysiologische Entitäten darstellen, auch wenn der klinische Nutzen zur Unterscheidung derzeit noch limitiert ist. Die Sonographie hat einen großen klinischen Nutzen bei der Unterscheidung der Entitäten.

\section{DGKCH-FV 06}

\section{Perforierte Appendizitis: Single Center Erfahrung mit >200 Patienten}

Tobias Klein', Daniel Diesbach', Thomas Boemers ${ }^{3}$

'Städt. Krankenanstalten Kinderkrankenhaus, Klinik für Kinderchirurgie und Kinderurologie, Köln, Deutschland; ${ }^{2}$ Kliniken der Stadt Köln gGmbH - Kinderkrankenhaus, Klinik für Kinderchirurgie und Kinderurologie, Köln, Deutschland; ${ }^{3}$ Kinderkrankenhaus Amsterdamer Straße Köln, Klinik für Kinderchirurgie \& Kinderurologie, Köln, Deutschland

Einleitung: Trotz einer flächendeckenden medizinischen Versorgung und der modernen diagnostischen Methoden kommt es heutzutage weiterhin bei rund 1/5 Patienten mit einer Appendizitis zu einer Perforation.

Fragestellung: Ziel der retrospektiven Datenanalyse ist die Evaluation der Patienten mit perforierter Appendizitis hinsichtlich des operativen Vorgehens (Single Port Appendektomie, laparoskopische oder offene Appendektomie, Konversionsrate) sowie des postoperativen Verlaufs (Dauer der Antibiotika-Therapie, Krankenhausverweildauer, Komplikationsrate). Zudem sollen die Ergebnisse der intraoperativen mikrobiologischen Abstriche hinsichtlich des Erregernachweises und des Antibiogramms analysiert werden.

Material und Methoden: Es erfolgte eine retrospektive Datenanalyse aller Patienten unter 18 Jahren, bei denen aufgrund des V.a. Appendizitis eine Appendektomie zwischen dem 01.01.2006 und dem 31.12.2016 in der Klinik für Kinderchirurgie und Kinderurologie des Kinderkrankenhauses Amsterdamer Straße durchgeführt wurde und bei denen sich intraoperativ eine perforierte Appendizitis zeigte. Ausgewertet wurden epidemiologische Daten, Operationsmethoden und -dauer, Konversionsraten, intraoperativer und histopathologischer Befund, postoperative Komplikationen sowie Krankenhausverweildauer. Des Weiteren wurden die intraoperativen mikrobiologischen Abstriche hinsichtlich des Erregerspektrums und der Antibiotikaresistenzlage analysiert.

Ergebnisse: Insgesamt wurden 225 Patienten mit einer perforierten Appendizitis in die Studie eingeschlossen. Mit $>70 \%$ war die Single-PortAppendektomie die häufigste durchgeführte Operationsmethode. Die Gesamtkomplikationsrate lag bei knapp über $20 \%$, wobei es sich Größtenteils um minor Komplikationen handelte. Die häufigsten Komplikationen waren Wundheilungsstörungen und Abszesse mit knapp 10\%. Eine Re-Operation war in unter 3\% der Fälle notwendig. Im Rahmen des Vortrags soll insbesondere differenzierter auf die mikrobiologische Situation bei einer perforierten Appendizitis eingegangen werden.
Schlussfolgerung: Trotz der verbesserten diagnostischen und therapeutischen Maßnahmen ist die perforierte Appendizitis weiterhin mit einer hohen Komplikationsrate vergesellschaftet. Eine entscheidende Maßnahme zur Reduktion der Morbidität ist die frühzeitige Diagnosestellung der akuten Appendizitis bevor es zu einer Perforation derselben kommt.

\section{Die akute Hüfte}

\section{DGKCH-FV 07}

Einseitige Epiphysenlösung des proximalen Femur rechts bei einem 2 Monate alten Säugling im Rahmen einer NAI - eine diagnostische und therapeutische Herausforderung.

\section{Birte Mack-Detlefsen', Domenica Merklein ${ }^{2}$, Medhat Demian', Thomas} Boemers $^{2}$

${ }^{1}$ Kinderkrankenhaus Amsterdamer Straße, Abteilung für Kinderchirurgie und Kinderurologie, Köln, Deutschland; ${ }^{2} \S \S \S$

Anamnese: Verlegung eines 2 Monate alten weiblichen Säuglings aus einer peripheren Kinderklinik mit unilateraler, anamnestisch atraumatischer massiver Schwellung des rechten Oberschenkels und Verdacht auf Kompartment zur weiteren kinderchirurgischen Versorgung.

Methodik: In der Inspektion zeigte sich eine deutliche Umfangsvermehrung des rechten Oberschenkels ohne sichtbares Hämatom oder Weichteilverletzung mit ausgeprägtem Druckschmerz, zusätzlich lag eine Schonhaltung des rechten Beines vor in Außenrotation und Abduktion. Zum Ausschluß einer entzündlichen Genese wurden eine Blutuntersuchung und eine Sonographie des rechten Oberschenkels und der rechten Hüfte durchgeführt.

Ergebnisse: In der Sonographie wurde der Verdacht auf eine corner fracture der Metaphyse des distalen Femurs rechts gestellt, zusätzlich zeigte sich die Muskulatur des rechten Oberschenkels massiv eingeblutet. Hinweise für eine Osteomyelitis fanden sich nicht. Die Röntgenuntersuchung bestätigte die corner fracture des Femurs rechts sowie eine weitere corner fracture der proximalen Tibia rechts. In der ergänzenden kontrastmittelgestützten MRT-Untersuchung beider Beine zeigte sich als Ursache der Schwellung und Fehlstellung eine Epiphysiolyse des proximalen Femurs rechts. Das Skelett-Screening zeigte zusätzlich eine Humerusschaftfraktur links, eine Fraktur der Costae 3-5 links und eine corner fracture der distalen Femurmetaphyse links. Im MRT des Schädels konnte ein bihemisphärisches Subduralhämatom nachgewiesen werden.

Eine Anamnese für das sicher mehrzeitige Trauma bestand seitens der Eltern nicht.

Die weitere Versorgung der Epiphysenlösung des proximalen Femur rechts stellte eine besondere Herausforderung dar. Nach ausführlicher Diskussion mit externen Kindertraumatologen und Kinderorthopäden wurde ein operatives Vorgehen favorisiert und eine Versorgung mittels K-Drähten durchgeführt.

Schlussfolgerung: An diesem Fallbespiel können 2 besondere Aspekte im Bereich der Kinderchirurgie dargestellt werden. Auf der einen Seite, die Fragestellung der Versorgung der Epiphysenlösung des proximalen Femur rechts, ob hinsichtlich des sehr jungen Alters in jedem Fall eine KDraht-Osteosynthese indiziert ist oder ob auch ein konservatives Vorgehen gerechtfertigt ist.

Zusätzlich der Aspekt der NAI mit der anfänglich ungewöhnlichen und unklaren Befundkonstellation, so dass erst nach umfassender Diagnostik das gesamte Ausmaß der Verletzungen dargestellt werden konnte und weitere Maßnahmen eingeleitet werden konnten. 


\section{Poster}

\section{DGKCH-PO 01 \\ Differences in the treatment of children with severe burns in Germany between burn centers and non-centers}

\section{Steffen Wahler', Pauline Wahler', Alfred Mueller ${ }^{3}$}

'keine, keine, Hamburg, Germany; ${ }^{2}$ St. Bernward GmbH, Epidemiologie, Hamburg; ${ }^{3}$ Analytic Services GmbH, Demographie, Munich, Germany

In Germany exist 19 defined centers dedicated for the care of children with severe burns. However, the transfer to such a center is neither enforced nor regulated in Germany. Thus, many children are treated in the next hospital available. The German quality report system allows insights, how many and how severely burned children are treated in centers and non-centers and how the treatments differ.

We examined federal statistics data and individual quality reports for all German hospitals for 2014 and 2015. Burn children, degree of burns, location, patient age, and coverage materials were analyzed. Results for burn centers and non-burn centers were compared. Software used for analysis was Microsoft Access ${ }^{\circledR} 2016$ and Excel $^{\circledR} 2016$.

In 2015 in Germany were 12,739 (2010: 11,604; 2005: 11,727) hospital stays reported with burn wounds on the body surface as primary diagnosis Thereof were 4335 (34.0\%) children under 5 years (2010: 3677 (31.7\%); 2005: $3605(30.7 \%)) .51 .3 \%$ of all burns were cared for in centers, $39.9 \%$ of degree $2 \mathrm{a}, 60.7 \%$ of degree $2 \mathrm{~b}$ and $71.1 \%$ of third degree. Burn coverages in children were performed in $56.3 \%$ in centers. $40.6 \%$ of second degree and $61.9 \%$ of third degree burns of the hands were cared for in centers. Centers perform $82 \%$ of skin transplants.

Coverage material overall used in centers is xenogeneic $13 \%$, alloplastic $32 \%$, combinations $12 \%$, hydrolytic absorbable membrane $42 \%$. Specifically in children, the hydrolytic absorbable membrane is most common with $48 \%$ of all coverages in non-centers and $53 \%$ in centers.

Despite Germany offers specific burn centers for children, around $50 \%$ of the children with a higher degree burn are treated outside the centers. Around a fifth of all specialized wound coverages, such as skin transplant or coverage with skin substituting materials, are performed outside the centers. The treatment patterns differ largely between centers and noncenters, except hydrolytic absorbable membrane is the preferred coverage choice in children in and outside of centers.

\section{DGKCH-PO 02}

\section{Vaginoplastiken bei jugendlichen Patientinnen mit weiblichen Genitalfehlbildungen}

Verena Ellerkamp' ', Katharina Rall'2, Sara Brucker', Jörg Fuchs'

'Uniklinikum Tübingen, Kinderchirurgie und Kinderurologie, Tübingen, Deutschland; ${ }^{2}$ Universitätsklinikum Tübingen, Gynäkologie, Tübingen, Deutschland

Einleitung: Operative Genitalkorrekturen bei komplexen weiblichen genitalen Fehlbildungen (Sinus urogenitalis, Kloakalfehlblidungen, Exstrophien) erfolgen meist innerhalb der ersten Lebensmonate-/jahre durch Kinderchirurgen oder Kinderurologen. In der Adoleszenz und z. T. auch erst im jungen Erwachsenenalter können gynäkologische Komplikationen, insbesondere Vaginalstenosen symptomatisch werden. Seit 2015 besteht eine enge kinderchirurgisch-gynäkologische Kooperation zum interdisziplinärenen operativen Management dieser Patientinnen.

Material und Methoden: Es erfolgte eine retrospektive Auswertung der Patientendaten, die von 2015 bis 2017 interdisziplinär kinderchirurgischgynäkologisch behandelt wurden. Die Fälle wurden unterschieden in primäre und sekundäre Korrekturen und hinsichtlich operativer Technik, postoperativer Vaginalweiter, Dauer eventueller vaginaler Bougierungen und Komplikationen ausgewertet.

Ergebnisse: Im genannten Zeitraum erhielten 21 Patientinnen eine Vaginoplastik. Das mediane Alter betrug zum Zeitpunkt der Vaginoplastik 19 Jahre (10-31 Jahre). Bei 12 Patientinnen handelte es sich um sekun- däre Vaginalkorrekturen nach im Median 4 vaginalen Voroperationen (range 1-7). 9 Patientinnen erhielten primäre Vaginoplastiken, davon 5 Patientinnen mit unkorrigiertem tiefem Uroginalsinus und 4 Patientinnen mit Vaginalatresie. Die Operationstechniken unterschieden sich wie folgt: partielle oder toatale urogenitale Mobilisation in Kombination mit einer Fortunoff-Plastik (14), laparoscopischer vaginaler Durchzug (3, Video Demonstration), Sigma vaginoplastik (2), modifizierte McIndoe-Vaginoplastik (2), Vecchieetti Vaginoplastik (1). Eine postoperative Dehnungstherapie über 12 Monate war bei 11/12 der Sekundärkorrekturen erforderlich und bei 5/9 der Primärkorrekturen. In einem medianen follow-up von 10 Monaten (8-34) besteht ein Fall mit Restenosierung, der aktuell noch mittels Bougierungstherapie handhabbar ist. Bei 20/21 Patientinnen besteht Kohabitationsfähigkeit bei physiologischen Vaginallängen und -weiten. Schlussfolgerung: Vaginalstenosen mit dem Problem der Dyspareunie können eine Spätkomplikation weiblicher genitaler Fehlbildungen nach weiblichen Genitalfehlbildungen sein. Das interdisziplinäre Management durch Kinderchirurgen und Gynäkologen ermöglicht eine optimale Behandlung der betroffenen Jugendlichen.

\section{DGKCH-PO 03}

\section{Stellenwert verschiedener bildgebender Verfahren in der Evaluation des Kurzdarmsyndroms bei Kindern}

Rasul Khasanov', Meike Weis', Kristina Maurer', Judith Felcht', Lucas Wessel'

'Medizinische Fakultät Mannheim der Universität

Heidelberg, Kinderchirurgische Klinik Mannheim, Mannheim, Deutschland;'²Medizinische Fakultät Mannheim der Universität Heidelberg, Institut für klinische Radiologie und Nuklearmedizin, Mannheim, Deutschland

Fragestellung: Zur Evaluation des Gastrointestinaltraktes bei Kindern mit Kurzdarmsyndrom stehen verschiedene radiologische Techniken zu Verfügung: konventionelle Röntgendiagnostik zur Evaluation der MagenDarm-Passage (MDP), MRT Sellink und Sonografie. Ziel dieser Arbeit ist der Vergleich dieser bildgebenden Techniken hinsichtlich der diagnostischen Güte zur Evaluation der Darmlänge (i), der Darmweite (ii) und der Detektion von Stenosen (iii).

Material und Methode: Es wurden retrospektiv die radiologischen und klinischen Daten von 8 Patienten evaluiert. Die Ergebnisse präoperativer radiologischer Diagnostik wurden hierfür mit intraoperativ erhobenen Befunden verglichen. Ausgewertet wurde die Vorhersagegenauigkeit einzelner Untersuchungsmodalitäten bezüglich verbliebener Darmlänge (i), der Darmweite (ii) und der Detektion von Stenosen (iii).

Ergebnisse: Die Darmlänge (i) konnte mit der Sonographie in 0 \% der Fälle korrekt vorhergesagt werden. In der MRT und in der MDP gelang die Vorhersage bei einer Darmlänge von $<30 \mathrm{~cm}$ sehr genau, bei $30-70 \mathrm{~cm}$ Darmlänge mit einer Fehlerquote von $\pm 25 \mathrm{~cm}$ und bei $>70 \mathrm{~cm}$ unzureichend. Die Darmweite (ii) konnte sowohl im Ultraschall mit $88 \%$, als auch in der MRT und der MDP $100 \%$ korrekt bestimmt werden.

Stenosen (iii) konnten im Ultraschall nicht dargestellt werden, in der MDP jedoch wurden $75 \%$ und in der MRT $100 \%$ der Stenosen detektiert.

Diskussion: Die Abdomensonografie stellt als kostengünstige Technik eine gute Screeningmethode für Kinder mit Kurzdarmsyndrom dar, um Veränderungen der Schlingenweite festzustellen. Zudem ist die Sonografie meist flächendeckend verfügbar. Jedoch ist hiermit lediglich eine orientierende Untersuchung möglich, auf die sich bei Verdacht auf Komplikationen weitere Diagnostik anschließen sollte. Die kontrastmittelgestütze, konventionelle Radiologie bietet unter Inkaufnahme der Röntgenstrahlung die Möglichkeit, Morphologie und Transportfunktion der Dünndarmschlingen gut darzustellen, stößt jedoch bei übereinanderprojizierenden Schlingen an ihre Grenzen. Die Magnetresonanztomografie (in Sellink-Technik) scheint die beste Methode zu sein, um die vorhandene Dünndarmsituation möglichst exakt abzubilden. Nachteile sind hier jedoch der relative hohe Zeitaufwand und eine für kleinere Kinder oft notwendige Anästhesie dar. Schlussfolgerung: Bei Kindern mit Kurzdarmsyndrom sollte eine Stufendiagnostik erfolgen: Die Evaluation der Darmweite gelingt sicher mit der Sonografie. Eine MRT Sellink bietet die Möglichkeit die intestinale Situa- 
tion präoperativ sicher abzubilden, ist jedoch nicht bei allen Kindern notwendig, da auch mit der MDP die Darmlänge und das Vorhandensein von Stenosen beurteilt werden kann.

\section{DGKCH-PO 04 \\ Anorektale Malformationen im Jugend- und Erwachsenenalter - Sind wir "Lost in Transition"? \\ Mark Malota, Stuart Hosie \\ Klinikum München-Schwabing, Kinderchirurgie, München, Deutschland}

Die operative Korrektur von Anorektalen Malformation (ARM) und des Morbus Hirschsprung (HD) ist eine Domäne der Neugeborenenchirurgie. Sowohl der Primäreingriff, als auch die Nachsorge werden zumeist in spezialisierten Zentren durchgeführt, da diese jungen Patienten eine kind- und jugendgerechte Begleitung benötigen, die von Problemen des Wachstums, der hormonellen Umstellung in der Pubertät bis hin zu psychosozialen Faktoren reicht.

Mit dem Erreichen der Adoleszenz und der Volljährigkeit jedoch, werden die Patienten oft zu „Erwachsenen-Proktologen“ transitioniert, für die diese postoperativen Folgezustände ein ungewohntes Terrain darstellen. Daraus resultiert oftmals eine beidseitige Frustration für Arzt und Patient und damit die Einstellung der oft notwendigen Nachsorge. Der Patient ist „Lost in Transition“.

Über standardisierte Fragebögen wurden Patienten der Jahrgänge 19902004, die eine Operation bei ARM oder HD erhalten haben, zu Transition und Behandlung in der Erwachsenen-Proktologie befragt.

Von 36 angeschriebenen Patienten antworteten 75 \% $(n=27)$. Fünfzehn Patienten hatten mehr als einmal den Proktologen gewechselt, weil sie unzufrieden mit der Behandlung waren, 6 davon mehr als zwei Mal. Neun Patienten gaben an, gar nicht mehr zum Proktologen zu einer Nachsorge zu gehen, davon litten 5 an behandlungsbedürftigen Strikturen im Analbereich. ARM und HD sind Erkrankungen des Neugeborenen und erfordern größere operative Eingriffe im Kindes- und Jungendalter. Um auch nach der Volljährigkeit eine gute Nachsorge gewährleisten zu können, ist neben einer suffizienten Transition auch die Kenntnis der gängigen Operationstechniken von Vorteil. Zudem brauchen wir Kinderchirurgen ein gutes Netzwerk zur Weiterbehandlung dieser Patienten, die ansonsten unnötig an den postoperativen Folgezuständen leiden.

\section{DGKCH-PO 05}

\section{Operationszeitpunkt Hodenhochstand - in trockenen Tüchern?}

\section{Ralf-Bodo Tröbs', Matthias Nissen ${ }^{2}$}

${ }^{1}$ Helios Klinikum Duisburg, Klinikum für Kinderchirurgie, Duisburg,

Deutschland; ${ }^{2}$ Marien Hospital Witten, St. Elisabeth Gruppe, Ruhr-Universität Bochum, Klinik für KInderchirurgie, Witten, Deutschland

Der Hodenhochstand ist die häufigste urogenitale Entwicklungsstörung des Jungen. Untersuchungen zur Entwicklung des Samenepithels zeigen, dass bei Abwarten über das 2. Lebensjahr hinaus zur sekundären Schädigung führt, woraus sich die Forderung ergibt, die Hoden bereits vorher an Sollstelle zu bringen. Im Kontrast dazu ergab eine Göttinger Studie von 2005 (Zöller, Ringert. Dt Ärztebl 2005;102: A 1750-A1752), dass weniger als $1 / 5$ der Jungen bis zum 2. Geburtstag operiert wurden!

Ziel der vorliegenden monoinstitutionellen Studie der Jahre 6/2015 bis 01/2018 ist eine aktuelle Überprüfung des Operationsalters.

Material und Methode: 134 Jungen wurden wegen eines Hodenhochstandes operiert und 129 Datensätze ausgewertet. Primärziel war das Alter zur Operation. Weiterhin erfassten wir Seitenverteilung, Höhe des Hodens sowie den chirurgischen Zugang. In Anlehnung an die Vor-Publikation bildeten wir drei Altersgruppen: Operation vor dem 2. Geburtstag, Alter 2 bis 4 Jahre; Alter 5 bis 16 Jahre.

Ergebnisse: Ein einseitiger Hochstand fand sich bei 76 (rechts 41 vs. links 35) und ein beidseitiger Hochstand bei 53 Jungen (41 \%). 55 Knaben wurden vor dem zweiten Geburtstag ( $43 \%$ ), 29 Jungen zwischen 2. und 4. Geburtstag (22\%), 42 im Alter von 5 bis 12 Jahren (33\%) und 3 Jungen wa- ren zwischen 12 und 16 Jahre alt (2\%). Laparoskopie kam in 8 Fällen zum Einsatz (davon 5 laparoskopischer Fowler-Stephens), wobei diese Jungen signifikant jünger waren als primär offen operierte $(p=0,023)$.

Diskussion: Die Beurteilung der Hodenposition beim Säugling und Kleinkind ist eine schwierige klinische Untersuchung, die Erfahrung, Gedult und Kontrollen erfordert. Die Zahlen belegen, dass mehr als die Hälfte der Kinder zu spät operiert wurde. Selbst unter Kalkulation einer Rate von $10 \%$ an sekundärem Hodenhochstand zeigt sich, dass die Problematik unterschätzt wird. Eine substantielle Verbesserung gegenüber den Göttinger Zahlen ist nicht zu erkennen.

Schlussfolgerung: Nur bei einem geringen Anteil der Patienten dürfte es sich um einen sekundären Hochstand handeln. Es gilt, das Problem-Bewusstsein zu schärfen und den einschlägigen Wissenstand bei Kindermediziner der verschiedenen Fachrichtungen weiterhin zu verbessern.

\section{DGKCH-PO 06 \\ Therapie der komplizierten Appendizitis: Drainage ja oder nein, dass ist hier die Frage}

Yannik Schmidt, Danielle Wendling, Dietrich von Schweinitz, Jochen Hubertus, Michael Berger

Universitätsklinikum München, Dr. von Haunersches Kinderspital, Kinderchirurgie, München, Deutschland

Hintergrund: Die kosteneffektivste und schonendste Behandlung ist für die komplizierte Appendizitis beim Kindern noch immer ungeklärt. Insbesondere für die Verwendung von Drainagen gibt es aktuell nur unzureichende Literatur.

Fragestellung: Welchen Stellenwert hat die Verwendung einer intraoperativ eingelegten Drainage nach Appendektomie bei der komplizierten Appendizitis im Kindesalter.

Material und Methoden: Retrospektive Analyse eines einzelnen Zentrums bezüglich aller im Zeitraum von 2013 bis 2018 operierten komplizierten Appendizitiden (Alter $<18$ Jahre). Hierfür wurde u. a. das präoperative Labor, der intraoperative Befund sowie die Histologie berücksichtigt. Für den intraoperativen Befund wurde jeweils zwischen gross spillage, minimal spillage, und no spillage unterschieden. Als Outcome-marker wurden die Komplikationsrate (Wundinfektionen, intraabdominelle Abszessbildung, u. a.) sowie die Krankenhausaufenthaltsdauer herangezogen.

Ergebnisse: Es wurden im genannten Zeitraum 59 Kinder mit komplizierter Appendizitis operiert. Hiervon wurden 7 Kinder offen (11,86\%) und 49 laparoskopisch $(83,05 \%)$ appendektomiert. Eine Konversion fand bei 2 Kindern (3,39\%) statt. In einem Fall (1,7\%) war die Operationstechnik unbekannt. Zwanzig Kinder (33,9\%) hatten gross spillage, 16 Kinder $(27,12 \%)$ minimal spillage, und 14 Kinder hatten no spillage (23,73\%). Bei $7(11,86 \%)$ konnte diese Information nicht exakt zugeordnet werden. In 2 Fällen $(3,39 \%)$ war diese Information nicht vorhanden. Vierundzwanzig Kinder erhielten eine Drainage (40,68 \%) und 33 Kinder erhielten keine (55,93\%). Bei 2 Kindern (3,39 \%) gab es diesbezüglich keine Information. Ob Kinder eine Drainage bekamen oder nicht korrelierte mit dem intraoperativen Befund. Kinder mit gross spillage bekamen in $65 \%$ der Fälle eine Drainage, bei minimal spillage 37,5 \% und bei no spillage nur $28,6 \%$. In keiner der Gruppen korrelierte die Verwendung einer Drainage mit einer reduzierten Komplikationsrate oder Krankenhausaufenthaltsdauer. Diskussion: In unserem Patientenkollektiv korreliert die Verwendung von Drainagen bei komplizierter Appendizitis nicht mit einer verminderten Komplikationsrate oder einer verringerten Krankenhausaufenthaltsdauer. 


\section{DGKCH-PO 07}

\section{Dieulafoy-Läsion als Ursache für eine schwere obere GI-Blutung des Neugeborenen}

\section{Frank-Mattias Schäfer', Christian Grillhös², Annette Kaiser ${ }^{3}$, Maximilian}

Stehr

'Cnopfsche Kinderklinik, Abteilung für Kinderchirurgie und Kinderurologie, Nürnberg, Deutschland; ${ }^{2} \mathrm{Cnopfsche} \mathrm{Kinderklinik,} \mathrm{Abteilung} \mathrm{für}$

Neonatologie und Pädiatrische Intensivmedizin, Nürnberg, Deutschland; ${ }^{3}$ Klinikum Nürnberg, Institut für Pathologie, Nürnberg

Einleitung: Die obere gastrointestinale Blutung des Neugeborenen ist eine sehr seltene Entität, über deren Inzidenz keine Daten existieren. Als häufige Ursachen werden das Verschlucken von mütterlichem Blut (als nur scheinbare kindliche Blutung), eine Kuhmilchproteinunverträglicheit sowie - als iatrogener Auslöser - die Platzierung einer nasogastrischen Sonde genannt. Seltene Ursachen umfassen vaskuläre Malformationen, Koagulopathien bzw. Gastritiden oder Ösophagitiden.

Material und Methoden: Wir berichten über ein weibliches Neugeborenes der 38.+3. SSW. Die postnatale Adaptation nach primärer Sectio gestaltete sich unauffällig. Bei der neonatologischen Erstversorgung wurde eine enorale Absaugung vorgenommen. Im Lebensalter von $24 \mathrm{~h}$ erbrach das Kind frischblutiges Sekret in zunehmender Menge, was zu einem Abfall des Hämoglobins von $15 \mathrm{mg} / \mathrm{dl}$ auf $9 \mathrm{mg} / \mathrm{dl}$ innerhalb von $2 \mathrm{~h}$ führte. Es erfolgte die Transfusion eines Erythrozytenkonzentrates sowie eine diagnostische Ösophagogastroduodenoskopie, bei der sich eine große, den Magen weitgehend ausfüllende Raumforderung an der Magenhinterwand zeigte, die in den Ösophagus hineinragte. In der daraufhin durchgeführten Minilaparotomie mit Gastrotomie stellte sich diese als Blutkoagel heraus. Nach Entfernen des Blutkoagels zeigte sich ein punktförmiges Ulcus an der Magenhinterwand. Es wurde eine Biopsie entnommen und das Ulcus übernäht. In der Folge erholte sich das Kind rasch, es kam zu keiner weiteren Blutung unter Therapie mit Omeprazol.

Ergebnisse: Die histologische Aufarbeitung der Biopsie zeigte das typische Bild einer Dieulafoy-Läsion des Magens mit einem oberflächlich im Ulcusgrund liegenden, starkkalibrigen und entzündlich alterierten Blutgefäß. Diskussion: Die Dieulafoy-Läsion ist eine seltene Gefäßanomalie im GITrakt, bei der eine kaliberstarke, geschlängelt verlaufende Arteriole oberflächlich in der dadurch ausgedünnten Mucosa liegt. Dadurch kommt es zu einer erhöhten Empfindlichkeit zur Ulcusbildung mit Blutungsneigung aufgrund einer Gefäßarrosion. Bei Kindern tritt dies jedoch sehr selten auf. Bislang sind ca. 30 Fallberichte publiziert, darunter nur zwei bei Neonaten in den ersten Lebenstagen/-stunden. In etwa der Hälfte der Fälle konnte die Blutungsquelle endoskopisch verifiziert und therapiert werden (Clipping, Epinephrininjektion), in den anderen Fällen wurde die Blutung, wie in unseren Fall, offen chirurgisch saniert. Eine grundsätzliche Empfehlung zur routinemäßigen Kontrollgastroskopie ist in diesen Fällen laut vorhandener Literatur nicht gegeben. In unserem Fall haben wir bislang bei beschwerdefreiem Kind darauf verzichtet.

Schlussfolgerung: Bei einer oberen GI-Blutung im Säuglings- und Kindesalter sollte auch das Vorliegen einer Dieulafoy-Läsion als spezielle Ulcusform in die Reihe der seltenen Differentialdiagnosen aufgenommen werden.

\section{Literatur \\ 1. Itani M, Alsaied T, Charafeddine L, Yazbeck N (2010) Dieulafoy's lesion in children. J Pediatr Gastroenterol Nutr 51(5):672-674 \\ 2. Koo YH, Jang JS, Cho JH et al Endoscopic injection treatment for gastric dieulafoy lesion in two newborn infants. Korean J :}

\section{DGKCH-PO 08 \\ Chirurgische Versorgung einer Ösophagusatresie mit unterer tracheoösophagealer Fistel bei einem extremen Frühgeborenen (510 g, 25 + 5 SSW) \\ Richard Wagner', Ulrich Thome', Martin Lacher \\ 'Uniklinikum Leipzig, Kinderchirurgie, Leipzig, Deutschland;'²Uniklinikum Leipzig, Neonatologie, Leipzig}

Hintergrund: Die chirurgische Therapie einer Ösophagusatresie (ÖA) bei extremen Frühgeborenen (ELBW; <1000 g) stellt eine Herausforderung dar. Der Versuch einer direkten Anastomosierung ist in der Literatur mit einer extrem hohen Mortalität verbunden. Wir berichten über ein extremes Frühgeborenes ( $510 \mathrm{~g}, 25+5 \mathrm{SSW}$ ) welches bei pränatal nicht bekannter ÖA und unterer tracheoösophagealer Fistel (TÖF; Typ Vogt IIIb) geboren wurde.

Verlauf: Nach intensivmedizinischer Stabilisierung und Intubation in der 1. Lebensstunde wurde die TÖF am 1. Lebenstag (LT) auf der Neonatologischen Intensivstation im Inkubator über einen extrapleuralen Zugang mittels Titan-Clip verschlossen (OP-Zeit $36 \mathrm{~min}$ ). Um das operative Trauma in der initialen Neonatalperiode zu reduzieren (Extubation nach 6 Tagen) entschlossen wir uns, die Gastrostomie erst am 22. LT bei $725 \mathrm{~g}$ Körpergewicht anzulegen. Nach stetiger Gewichtszunahme ( $2510 \mathrm{~g})$ erfolgte im Alter von 3 Monaten eine thorakoskopische Ösophagusanastomosierung (OP-Zeit $93 \mathrm{~min}$ ). Im Verlauf musste die Anastomose insgesamt $8 \times$ endoskopisch dilatiert werden.

Ergebnis: Aktuell schluckt die Patientin im Alter von 2 Jahren problemlos und gedeiht gut $(9,9 \mathrm{~kg}(\mathrm{P} 6) ; 80 \mathrm{~cm}(\mathrm{P} 2))$. Problematisch sind 1-3 Atemwegsinfekte pro Jahr im Sinne einer pneumologischen Begleitmorbidität. Schlussfolgerung: Unser Fall zeigt, dass eine limitierte Operation direkt nach Geburt mit ausschließlichem Fistelverschluss via Thorakotomie, sekundärer Gastrostomie und Anastomosierung im Verlauf (Gewicht $>2,5 \mathrm{~kg}$ ) eine gute Alternative bei ÖA mit TEF bei extremen Frühgeborenen sein kann.

\section{DGKCH-PO 09}

\section{Fetal growth of infants with esophageal atresia}

Ralf-Bodo Tröbs' ${ }^{1}$ Ulrich Witzel' ${ }^{2}$, Andreas Lipphaus ${ }^{3}$

${ }^{1}$ Helios Klinikum Duisburg, Klinik für Kinderchirurgie, Duisburg, Germany; ${ }^{2}$ Ruhr-Universität Bochum, Lehrstuhl für Produktentwicklung Fakultät für Maschinenbau, Bochum, Germany; ${ }^{3}$ Fakultät für Maschinenbau, Ruhr-Universität Bochum, Lehrstuhl für Produktentwicklung Fakultät für Maschinenbau, Bochum, Germany

In the majority of cases with esophageal atresia (EA) the esophagus ends blindly. In addition, a tracheoesophageal fistula (TEF) of the lower part of the esophagus is present. In affected fetuses swallowing of amniotic fluid is interrupted and polyhydramnios results. The aim of the presented analysis is to compare the prenatal growth of infants with EA and TEF with a control group. We tested the hypothesis, that intrauterine gain of weight (growth) of fetuses with EA and TEF is impaired in comparison with neonates with a normal gastrointestinal tract.

Method: Birth weights (BW) of 52 newborns with EA and TEF (29 male) were compared with a control group of 193 infants (140 male) operated on for inguinal hernia (Hernia). Regression analysis was applied to describe growth curves.

Results: Comparison of birth weights generally did not reveal any significant differences. Multivariate regression analysis revealed a relevant difference between both groups $(-2,83, p=0,005,<0,001)$. The regression coefficient was -195.27 .

Discussion: Infants with EA showed a slight but statistical relevant growth retardation. We found a mean difference of BW of $195 \mathrm{~g}$. It is well known, that fetuses are "breathing" (without gas exchange) during REM sleep. It can be hypothesized, that intrauterine breathing (aspiration) of amniotic fluid (AF) leads to a passage of AF via TEF. Conclusion. The trophic effect of $\mathrm{AF}$ might compensate the negative influence of impaired esophageal passage during fetal life. 


\section{DGKCH-PO 10}

Präpartal Verdacht auf Zwerchfellhernie links - postpartal Serpentine-like-Syndrom, eine besondere neonatologische und kinderchirurgische Herausforderung

Birte Mack-Detlefsen, Lena Gindner, Tobias Klein, Thomas M. Boemers Kinderkrankenhaus Amsterdamer Straße, Abteilung für Kinderchirurgie und Kinderurologie, Köln, Deutschland

Falldarstellung eines weiblichen Neugeborenes der 38. SSW mit präpartal diagnostiziertem Polyhydramnion und V.a. Zwerchfellhernie links. $\mathrm{Da}$ die postpartale Bildgebung keinen eindeutigen Befund ergab wurde die Indikation zur medianen Oberbauchlaparotomie gestellt. Intraoperativ zeigt sich ein Brachyösophagus mit ins Mediastinum prolabiertem Magen, Dünn- und Dickdarm sowie eine Asplenie. In Zusammenschau dieser Befunde und dem Nachweis einer Rachischisis im MRT der HWS wurde die Diagnose eines „Serpentine like“-Syndroms gestellt. Der weitere Verlauf gestaltete sich sehr kompliziert. Aufgrund der ausgeprägten Enge des Spinalkanals erfolgte eine neurochirurgische Laminektomie mit Dekompression des zervikalen Myelons am 17. Lebenstag. Im weiteren intensivmedizinischen Verlauf entwickelte die Patientin eine ausgeprägte Magenentleerungsstörung, die zwei Relaparotomien erforderlich machte. Zunächst erfolgte eine Seit-zu-Seit-Duodenojejunoastomie, 14 Tage später eine Y-Roux-Anastomose mit Jejunostomaanlage. Aktuell ist die Patientin nach einem fast 5-monatigen Krankenhausaufenthalt mit zuletzt erneuter Relaparotomie mit einem Re-Verschluss des Zwerchfelldefektes mittels Goretex-Patch und Anlage einer Gastrojejunostomie nachhause entlassen. Die Ernährung erfolgt über das Jejunostoma, der Magensondenrückstau, der zum Teil resondiert wird, beträgt bis zu $200 \mathrm{ml}$ pro Tag.

Falldiskussion: Weltweit sind einschließlich unserer Patientin 9 Fälle mit "Serpentine-like“ Syndrom bekannt. Keiner der Patienten hat bisher das Vorschulalter erreicht. Die Prognose unserer Patientin bleibt ebenso unklar sowie das weitere Management. Allerdings ist der bisherige Verlau unserer Patientin im Hinblick auf dieses extrem seltene Syndrom relativ erfreulich. Anzumerken bleibt noch, dass im Rahmen der Pränataldiagnostik einmalig der V.a. ein „Serpentine-like“ Syndrom geäußert wurde, jedoch nicht weiter verfolgt wurde. Sicherlich für die Eltern keine ganz unwichtige Information.

\section{DGKCH-PO 11}

\section{Merkoniumtransportstörung des sehr kleinen Frühgeborenen als interdisziplinäres Problem - präventive Strategie zur Mekoniummobilisation}

Karin Rothe', Jenyffer-Tabea Schröder', Lars Garten ${ }^{3}$

'Charite - Universitätsmedizin Berlin, Klinik für Kinderchirurgie - Leitung,

Berlin, Deutschland; ${ }^{2}$ Charite - Universitätsmedizin Berlin, Klinik für

Kinderchirurgie, Berlin, Deutschland; ${ }^{3}$ Charité - Universitätsmedizin Berlin, Klinik für Neonatologie, Berlin, Deutschland

Eine verzögerte Entleerung von Mekonium tritt häufig bei ELBW- und hypotrophen VLBW-Frühgeborenen auf und ist mit einem hohen Risiko für gastrointestinale Komplikationen vergesellschaftet $[1,2]$.

Durch die frühe und konsequente Mobilisation des eingedickten Mekoniums kann oftmals eine chirurgische Intervention vermieden werden [3-5]. Zielstellung: Entwicklung einer SOP zum standardisierten Vorgehen zur Vermeidung einer manifesten Mekoniumobstruktion/Mekoniumileus mit Notwendigkeit einer operativen Entfernung des intraenteralen Mekoniums bei ELBW- und hypotrophen Frühgeborenen (GG $<3$. Perzentile) mit einem Gewicht zwischen 1000 und $1499 \mathrm{~g}$.

Ergebnisse: Stufe 1 - RAUS-Manöver: Start 1. Lebenstag.

Versuch der Mobilisation von eingedicktem Mekonium durch „Rektales Anspülen unter Sonographiekontrolle _ RAUS “

Filmsequenz-dokumentation mittels Loop-Funktion

Zum Anspülen wird verwendet: aufgewärmte $\mathrm{NaCl}$ 0,9 \% Lösung (5-10$20 \mathrm{ml})+$ ein halbes Babylax ${ }^{\oplus}(=0,25 \mathrm{mg}$ Glycerol).

Angespült wird entweder mit einem kurzem Darmrohr [Rectal tube $10 \mathrm{~cm}$ Size 5 mm, Ref. 431100 (Fa. Rüsch-Teleflex)] oder mittels dünnem „wei- chen" Blasenkatheter [Soft rubber, Size 2,0 mm/CH6 Ref. 404300 (Fa. Rüsch-Teleflex)].

Frequenz des Anspülens: 8-12 stdl. Dokumentation in Copra

Hinweis: Das RAUS-Manöver wird bei ELBW-Frühgeborenen unter $750 \mathrm{~g}$ Geburtsgewicht nur von einem Facharzt durchgeführt.

Stufe 2: zusätzlich ab 4. Lebenstag bei unzureichender Mekoniumentleerung trotz RAUS-Manöver.

Polyethylengykol (Movicol ${ }^{\bullet}$ aromafrei JUNIOR) per Magensonde. Ein Beutel Movicol ${ }^{\circledR}$ aromafrei enthält 6,9 $\mathrm{g}$ Pulver, dies wird in 62,5 ml Aqua dest. aufgelöst (fertige Lösung enthält dann $0,11 \mathrm{~g} / \mathrm{ml}$ ). Dosierung: $2 \times 0,5-$ $1,0 \mathrm{~g} / \mathrm{kg} / \mathrm{d}$ p. o.; Gabe erfolgt zwischen den Mahlzeiten.

Stufe 3: Zusätzlich zu erwägen ab ca. 7. Lebenstag; Kontrastmittel (z. B. Accupaque $300^{\circ}$ oder Imeron $300^{\circ}$ ) per Magensonde 1-2 ml/kg KG, Erfolgt auf obligat oberärztliche Anordnung in Rücksprache mit der Kinderradiologie und Kinderchirurgie. Ziel: therapeutische (abführende) Wirkung und Diagnostik (Stenose? Perforation?)

Ergebnisse und Diskussion: Seit Einführung der SOP ist ein deutlicher Rückgang an chirurgischen Interventionen bei ELBW- und hypotrophen VLBW Frühgeborenen nachweisbar. Ärzte und Pflegende fühlen sich gegenseitig gestärkt und ernst genommen in der Verantwortung durch nichtinvasive konservative Maßnahmen zur Patientensicherheit und Qualitätsverbesserung beizutragen.

\section{Literatur}

1. McCowan L, Horgan RP (2009) Risk factors for small for gestational age infants. Best Pract Res Clin Obstet Gynaecol 23(6):779-793

2. Tsukamoto H, Fukuoka H, Koyasu M et al (2007) Risk factors for small for gestational age. Pediatr Int 49(6):985-990

\section{DGKCH-PO 12}

\section{Diagnosewandel des akuten Abdomens beim VLBW-} Frühgeborenen von 1995 bis heute

Tabea Schröder, Johanna Buttenberg, Karin Rothe

Charité - Universitätsmedizin Berlin, Klinik für Kinderchirurgie, Berlin, Deutschland

Hintergrund: Das akute Abdomen beim VLBW-Frühgeborenen ist eine gefürchtete Komplikation, welche mit einer hohen Morbidität und Mortalität einher geht.

Fragestellung: Ergibt sich ein Wandel in den Diagnosen und im outcome bei den VLBW-Frühgeborenen dadurch, dass das Gestationsalter und das damit verbundene Geburtsgewicht immer weiter sinkt?

Material und Methoden: In unsere Untersuchung wurden alle VLBWFrühgeborenen eingeschlossen, die im Zeitraum vom 01.01.1995 bis 31.12.2017 in der Klinik für Neonatologie der Charite - Universitätsmedizin Berlin aufgrund eines akuten Abdomens operiert werden mussten. Ausgeschlossen wurden Kinder mit angeborenen Fehlbildungen des Gatrointestinaltraktes, wobei Kinder mit einer Rotationsstörung des Darmes eingeschlossen wurden.

Ergebnisse: Bei den in unserem Patientenkollektiv aufgetretenen Erkrankungen handelt es sich um NEC, FIP, Mekoniumileus, Volvulus und Milk Curd sowie sonstige Ursachen eines Ileus.

Insgesamt wurden in die Untersuchung 172 Kinder eingeschlossen. Es zeigt sich, dass die Anzahl der operierten Kinder im Zeitraum von 19952004 im Vergleich zu den Folgejahren 2005-2017 höher war. Die Kinder waren im Beobachtungszeitraum von 2005-2017 erwartungsgemäß deutlich unreifer und leichter als im dem Zeitraum von 1995-2004. Trotzdem veränderte sich die Verteilung der Diagnosen statistisch nicht signifikant. Die häufigste Erkrankung war die NEC mit 43 \% (1995-2004), bzw. 48 \% (2005-2017).

Es zeigte sich dass obwohl die Kinder immer unreifer wurden, die Letalität konstant blieb mit 26 \% im Zeitraum von 1995-2004 im Vergleich zu 25 \% im Zeitraum von 2005-2017. Jedoch stieg die Anzahl der komplizierten Verläufe mit der Indikation zur Revision von $24 \%$ auf $41 \%$.

Diskussion/Schlussfolgerung: Aufgrund der Fortschritte in der Medizin ist ein Überleben von immer unreiferen Kindern möglich geworden. Das 
akute Abdomen ist und bleibt eine gefürchtete Komplikation. Insgesamt scheint in unserer Klinik die Inzidenz des operationsbedürftigen akuten Abdomens zu sinken, bei konstanter Mortalität in dieser Patientengruppe über die Jahre scheint aber die prozentuale Morbidität zu steigen.

Die Vermeidung des akuten Abdomens ist eine wichtige Voraussetzung zur Verbesserung des outcome des VLBW-Frühgeborenen. 


\title{
Abstracts der 41. Jahrestagung des Berufsverbandes Kinderkrankenpflege Deutschland (BeKD)
}

\author{
Wissenschaftliche Leitung
}

Elfriede Zoller

\section{Poster}

\section{BeKD-PO 01 \\ Frühe Hilfen als neues Arbeitsfeld der Kinderkrankenpflege: Erste Ergebnisse einer empirischen Untersuchung im Rahmen einer Dissertation zu Familien-Gesundheits- und Kinderkrankenpflegekräften \\ Birte Kimmerle', Wilfried Schnepp ${ }^{2}$, Friederike zu Sayn-Wittgenstein ${ }^{3}$ 'Universität Witten/Hedecke, Fakultät für Gesundheit - Department Pflegewissenschaft, Beuren, Deutschland; ${ }^{2}$ Universität Witten/Herdecke, Fakultät für Gesundheit, Department für Pflegewissenschaft, Witten, Deutschland; ${ }^{3}$ Hochschule Osnabrück, Fakultät Wirtschafts- und Sozialwissenschaften, Osnabrück, Deutschland}

Fragestellung: Für Gesundheits- und Kinderkrankenpflegekräfte stellen die „Frühen Hilfen“ ein neues Arbeitsfeld dar, in dem sie als FamilienGesundheits- und Kinderkrankenpflegekräfte (FGKiKP) tätig sind. Eingebunden in Netzwerke Früher Hilfen sollen sie dazu beitragen, dass Kinder aus benachteiligten und belasteten Familien gesund und gewaltfrei aufwachsen. Doch wer sind diese Fachkräfte, was machen sie und was daran ist neu? Hierauf will die vorliegende Arbeit Antworten geben und beleuchtet die Situation von FGKiKP.

Material und Methode: Im Rahmen der Untersuchung werden Gesundheits- und Kinderkrankenpflegekräfte befragt, die als FGKiKP tätig und qualifiziert sind. Der Kontakt zu den Interviewpartner*innen erfolgt über die Geschäftsstelle des Berufsverband Kinderkrankenpflege Deutschland e. V. (BeKD) und über regional ausgewählte Arbeitsstellen von FGKiKP sowie über Weiterbildungsanbieter. Acht offene, leitfadengestützte Interviews sowie eine Gruppendiskussion mit Angehörigen der Berufsgruppe stellen die empirische Basis der ersten Analyse der Situation von FGKiKP dar. Die Arbeit folgt den Vorgehensweisen der interpretativen Sozialforschung, im Speziellen dem Vorgehen der Grounded Theory (nach Strauss 1998), ergänzt durch den neueren Ansatz der Situationsanalyse (Clarke, Friese und Washburn 2018).

Ergebnisse: Es handelt sich um erfahrene Pflegekräfte, die als Pionierinnen in einem neuen Tätigkeitsfeld gefordert sind „sich selbst zu entwerfen“. In der Zusammenarbeit mit anderen Disziplinen befinden sie sich in fortwährenden Aushandlungsprozessen. Sie sind bestrebt ihre Expertise zur Geltung zu bringen, sich zu positionieren und strukturell zu verankern. Gleichzeitig handelt es sich größtenteils um befristete Arbeitsverhältnisse mit geringer Entlohnung und großen Abhängigkeiten. Die Situation dieser spezifischen Fachkräfte ist nicht ausreichend geklärt.

Diskussion: Auf der einen Seite stellt die Tätigkeit in den Frühen Hilfen hohe Anforderungen an die Berufsgruppenangehörigen und fordert großes Engagement vonseiten jeder einzelnen Fachkraft verbunden mit einem gestiegenen Qualifikationsprofil. Auf der anderen Seite sind die Beschäftigungsbedingungen unbefriedigend. Hinzu kommt, dass weit mehr Gesundheitsfachkräfte in den Frühen Hilfen gebraucht werden, als seither dort arbeiten (Pabst et al. 2018). Diskutiert werden soll darum, was die Perspektive für die Berufsgruppe sein sollte.

Schlussfolgerung: Die Analyse der Situation von Gesundheits- und Kinderkrankenpflegenden in Frühen Hilfen soll Aufschlüsse darüber geben, welche Rolle die Berufsangehörigen in den Frühen Hilfen innehaben und bezieht dabei mit ein, unter welchen Bedingungen und gesellschaftlichen Strukturen sich die Kinderkrankenpflege dort entwickelt. Vor dem Hintergrund dieser ersten Erkenntnisse sollen die Chancen aber auch Prämissen für die Tätigkeit der Familien-Gesundheits- und Kinderkrankenpflege aufgezeigt werden.

\section{BeKD-PO 02}

Das Erleben der stetigen Pflegepräsenz in der außerklinischen Kinderintensivpflege aus den Perspektiven von Eltern und Pflegenden

Sandra Falkson, Maren Roling

Universität Witten/Hedecke, Pflegewissenschaft, Witten, Deutschland

Hintergrund: In den letzten Jahren stieg die Zahl pflegebedürftiger Kinder und Jugendlicher, die zu Hause gepflegt werden, stetig an. Die außerklinische Intensivpflege ist eine spezialisierte Dienstleistung mit dem Ziel, technologieabhängige Kinder in häuslicher Umgebung zu pflegen und die Familien zu begleiten. Durch die häusliche Intensivpflege erhalten die Kinder die Möglichkeit, in ihrem vertrauten familiären Umfeld aufzuwachsen und am gesellschaftlichen Leben teilzuhaben. Kinder, die zu Hause gepflegt werden, weisen gegenüber Kindern, die in Institutionen leben, Entwicklungsvorteile auf. Die Pflege in der häuslichen Umgebung unterstützt die eigene Identitätsentwicklung der Kinder. Trotz der benannten Vorteile, die die außerklinische Kinderintensivpflege für die Kinder und ihre Familien mit sich bringt, muss die Konsequenz mitbedacht werden, dass stets eine professionelle Pflegeperson in der Familie präsent ist.

Methodik: Das qualitative Forschungsdesign richtete sich nach der Grounded Theory Methode. Die Datenerhebung erfolgte durch halbstrukturierte, leitfadengestützte Interviews mit 14 Eltern von technologieabhängigen Kindern und 14 Pflegepersonen.

Ergebnisse: Für die Eltern steht das Phänomen „vertrauen können“ im Vordergrund. Sind sie im Stande zu vertrauen, können sie das Leben mit den Pflegenden in der Häuslichkeit akzeptieren. Eine Strategie die Eltern dazu anwenden, ist das genaue Beobachten der Pflegenden. Können Pflegende Klinikroutinen ablegen und die häuslichen und familiären Routinen respektieren, gelingt es den Eltern zu vertrauen, sodass die Pflegenden trotz ihrer stetigen Präsenz eine Entlastung darstellen.

Im Fokus der Pflegenden steht der zentrale Aspekt „sich einlassen können“. Da die Pflegenden für viele Stunden in den Familien arbeiten, setzt diese spezielle Situation bei ihnen Unvoreingenommenheit und Loyalität voraus. Können sie sich auf die häusliche Situation einlassen, kann eine respektvolle Pflegebeziehung aufgebaut werden. Neben medizinischem Wissen ist eine stabile Persönlichkeit, Belastbarkeit und Flexibilität eine wichtige Voraussetzung für Ihre Arbeit. Die Möglichkeit Positives in den 
Familien bewirken zu können, führt zu Zufriedenheit der Pflegepersonen. Hierzu benötigen die Pflegenden Reflexionsvermögen und Kommunikationskompetenzen.

Fazit: Die außerklinische Intensivpflege stellt eine gute Möglichkeit für die Familien dar ihr Kind zu Hause zu versorgen. Dennoch fehlt es den Eltern sowohl an pflegerischer Anleitung als auch an mentaler Vorbereitung, damit sie in der Lage sind die stete Präsenz der Pflegenden und die damit einhergehende fehlende Privatsphäre besser akzeptieren zu können. Hinsichtlich ihrer Qualifikationen fühlten sich die Pflegenden unzureichend auf die Versorgung technologieabhängiger Kinder in der außerklinischen Intensivpflege vorbereitet. Wünschenswert wäre dieses besondere Pflegesetting mit seinen spezifischen Herausforderungen in der Ausbildung näher in den Fokus zu nehmen. 


\section{Autorenverzeichnis}

A

\begin{tabular}{|c|c|}
\hline \multirow{2}{*}{\multicolumn{2}{|c|}{$\begin{array}{l}\text { Abel, Florian } \\
\text { Abellan-Schneyder, }\end{array}$}} \\
\hline & \\
\hline Francisca & DGKJ-PO 43 \\
\hline Adelmann, Sarah & DGSPJ-PO 30 \\
\hline Akçay, Marina & DGSPJ-PO 07 \\
\hline Akmatov, Manas & DGSPJ-PO 18 \\
\hline Alberer, Martin & DGKJ-FV 05 \\
\hline Albrecht, Melanie & DGKJ-FV 24 \\
\hline Allen, Kerstin & DGKJ-PO 45 \\
\hline André, Rupp & DGSPJ-FV 01 \\
\hline Apfelbacher, & \\
\hline Christian & $\begin{array}{l}\text { DGKJ-FV 08, DGKJ-FV } 18 \text {, } \\
\text { DGKJ-PO 05, DGKJ-PO } 23\end{array}$ \\
\hline Apitz, Christian & DGKJ-FV 25, DGKJ-PO 34 \\
\hline Artelt, Tanja & DGSPJ-PO 13 \\
\hline Ascherl, Rudi & DGKJ-PO 24 \\
\hline Asenbauer, Elisabeth & DGKJ-PO 62 \\
\hline Atzendorf, Josefine & DGKJ-PO 23 \\
\hline auf dem Brinke, Elena & DGKJ-PO 30 \\
\hline Augustin, Michaela & DGSPJ-PO 03 \\
\hline Aust, Daniela & DGKJ-PO 07 \\
\hline Avrami, Georgia & DGKJ-PO 29 \\
\hline
\end{tabular}

\section{B}

\begin{tabular}{|c|c|}
\hline Ballauff, Antje & DGKJ-PO 08, DGSPJ-PO 30 \\
\hline Barth, Michael & DGKJ-PO 48 \\
\hline Bätzing, Jörg & DGSPJ-PO 18 \\
\hline \multicolumn{2}{|l|}{ Bau, Anne- } \\
\hline Madeleine & DGSPJ-PO 34 \\
\hline Bauer, Antonia & DGKJ-PO 06 \\
\hline Baysal, Fatma & DGKJ-PO 42 \\
\hline Beck, Ricardo & DGKJ-PO 13 \\
\hline Becker, Marinne & DGSPJ-PO 42 \\
\hline Beck-Wödl, Stefanie & DGKJ-FV 20 \\
\hline Bedrosian, Camille & DGKJ-PO 46 \\
\hline Beer, Angelina & DGKJ-PO 13 \\
\hline Beier, Rita & DGKJ-PO 14 \\
\hline Beisenherz, Christina & DGSPJ-PO 09 \\
\hline Bendfeldt, Hanna & DGKJ-PO 50 \\
\hline Bergelt, Corinna & DGSPJ-PO 39 \\
\hline Berger, Michael & DGKCH-PO 06 \\
\hline Bernbeck, Benedikt & DGKJ-FV 09, DGKJ-PO 09 \\
\hline \multicolumn{2}{|l|}{ Berndt-Müller, } \\
\hline Marcella & DGKJ-PO 48 \\
\hline Berthold, Daniel & DGKJ-FV 02 \\
\hline Bertsche, Thilo & DGSPJ-PO 25 \\
\hline Bertsche, Astrid & DGSPJ-PO 25 \\
\hline Beyer, Florian & DGKJ-PO 73 \\
\hline Bickes, Marie Sophie & DGKJ-FV 24 \\
\hline Bielack, Stefan & DGSPJ-PO 17 \\
\hline Bien, Sarah & DGSPJ-PO 04 \\
\hline Birdir, Cahit & DGSPJ-FV 08 \\
\hline Blank, Lydia & DGKJ-FV 01 \\
\hline Blank, Rainer & DGKJ-FV 01 \\
\hline Blankenburg, Ulrike & DGKJ-FV 04 \\
\hline Boek, Friederike & DGKJ-FV 04 \\
\hline \multirow[t]{2}{*}{ Boemers, Thomas } & DGKCH-FV 06, DGKCH-FV \\
\hline & 07, DGKCH-PO 10 \\
\hline Boesten, Tineke & DGSPJ-PO 17 \\
\hline
\end{tabular}

\begin{tabular}{|c|c|c|c|}
\hline Böhne, Carolin & DGKJ-FV 24 & Diesbach, Daniel & DGKCH-FV 06 \\
\hline Bohnhorst, Bettina & DGKJ-FV 23 & Digweed, Dena & DGKJ-PO 50 \\
\hline Bokemeyer, Carsten & DGSPJ-PO 17 & Dittmann, Falko & DGSPJ-PO 05 \\
\hline Bollow, Esther & DGSPJ-PO 42 & Dittrich, Annika & DGKJ-FV 18 \\
\hline Borchert, Kathrin & DGKJ-PO 65 & Döbler, Marion & DGSPJ-FV 03 \\
\hline Bornemann, & & Döring, Stephan & DGKJ-FV 08 \\
\hline Reinhard & DGKJ-FV 06 & Dresbach, Till & DGKJ-PO 55, DGSPJ-PO 15 \\
\hline Borusiak, Peter & DGSPJ-FV 02 & Du, Yong & DGSPJ-PO 40 \\
\hline Bösch, Annemarie & DGKJ-PO 52 & du Bois, Florian & DGKJ-PO 03 \\
\hline Boschann, Felix & DGKJ-PO 52 & Dubois, A.E.J. & DGKJ-FV 27 \\
\hline Böse-O'Reilly, & & Dubrall, Diana & DGSPJ-PO 16 \\
\hline Stephan & DGKJ-PO 62 & Dunstheimer, & \\
\hline \multirow[t]{2}{*}{ Böswald, Michael } & DGKJ-PO 26, DGKJ-PO 71, & Désirée & DGKJ-FV 17 \\
\hline & DGKJ-PO 73 & Duppel, Uta & DGKJ-PO 64 \\
\hline $\begin{array}{l}\text { Bous, Michelle } \\
\text { Brandstetter, }\end{array}$ & DGKJ-PO 57 & & \\
\hline \multirow[t]{2}{*}{ Susanne } & DGKJ-FV 08, DGKJ-FV 18, & E & \\
\hline & DGKJ-PO 05, DGKJ-PO 23 & Eberl, Wolfgang & DGKJ-PO 16 \\
\hline Braun, Christian Karl & DGKJ-PO 33 & Ebinger, Martin & DGKJ-PO 09 \\
\hline Braun, Katrin & DGKJ-PO 42 & Effner, Renate & DGKJ-PO 27 \\
\hline Braun, Sebastian & DGKJ-PO 65 & Ehehalt, Stefan & DGSPJ-FV 07 \\
\hline Braunschweig, Till & DGKJ-PO 15, DGKJ-PO 17 & Eichinger, Michael & DGSPJ-PO 28, DGSPJ-PO 43 \\
\hline Brecht, I.B. & DGKJ-PO 09 & Eiffert, Helmut & DGSPJ-PO 13 \\
\hline Brenner, Sebastian & DGKJ-PO 13 & Ellerkamp, Verena & DGKCH-PO 02 \\
\hline Bretthauer, & & Ernst, Christian & DGKJ-FV 28 \\
\hline Sebastian & DGSPJ-PO 15 & Ernst, Julia & DGSPJ-PO 36 \\
\hline Breuer, Johannes & DGKJ-PO 35, DGKJ-PO 70 & Eschrich, Gabrielle & DGSPJ-PO 17 \\
\hline Brockow, Inken & DGKJ-FV 14 & Eschweiler, Jacob & DGKJ-PO 18 \\
\hline Brucker, Sara & DGKCH-PO 02 & Ewald, Dominik A. & DGKJ-PO 68 \\
\hline Brunder, Peter & DGKJ-FV 02 & Eyerich, Kilian & DGKJ-PO 27 \\
\hline Buchert, Rebecca & DGKJ-FV 20 & Eyermann, Richard & DGKJ-PO 36, DGKJ-PO 47, \\
\hline Bührer, Christoph & DGKJ-FV 22, DGKJ-PO 52 & & DGKJ-PO 49, DGKJ-PO 58, \\
\hline Buschmann, Anke & DGSPJ-PO 05 & & DGKJ-PO 72 \\
\hline Büttel, Hans-Martin & DGKJ-PO 44 & & \\
\hline \multirow[t]{3}{*}{ Buttenberg, Johanna } & DGKCH-PO 12 & & \\
\hline & & $F$ & \\
\hline & & Farin-Glattacker, Erik & DGSPJ-PO 38 \\
\hline C & & Faust, Johannes & DGKJ-PO 26 \\
\hline Calaminus, Gabriele & DGKJ-FV 09, DGSPJ-PO 17 & Fecarotta, Simona & DGKJ-PO 45 \\
\hline Carai, Susanne & DGSPJ-PO 14 & Fegeler, Ulrich & DGKJ-FV 07 \\
\hline Casaulta, Carmen & DGKJ-PO 67 & Fehlhaber, Beate & DGKJ-FV 24 \\
\hline Chao, Cho-Ming & DGKJ-FV 02 & Fehr, Folkert & DGKJ-PO 68, DGKJ-PO 69 \\
\hline Cimms, Tricia & DGKJ-PO 51 & Felcht, Judith & DGKCH-PO 03 \\
\hline Classen, Martin & DGSPJ-PO 30 & Feldhahn, Lutz & DGSPJ-PO 42 \\
\hline \multirow[t]{3}{*}{ Costard, Sylvia } & DGSPJ-PO 06 & Fenz, Diana & DGSPJ-PO 13 \\
\hline & & Fiedler, Barbara & DGKJ-PO 25 \\
\hline & & Flasche, Stefan & DGSPJ-PO 11 \\
\hline D & & Florian, Annamaria & DGKJ-PO 17 \\
\hline Däbritz, Jan & DGSPJ-PO 31, DGSPJ-PO 32 & Föll, Dirk & DGSPJ-PO 29 \\
\hline Daikeler, Thomas & DGKJ-PO 20 & Förster, Reinhold & DGKJ-FV 24 \\
\hline Dame, Christof & DGKJ-FV 22, DGKJ-PO 52 & Frank, Thomas & DGKJ-PO 26, DGKJ-PO 71 \\
\hline Daniel, Eleni & DGKJ-PO 50 & Franssen, Meike & DGKJ-PO 71 \\
\hline Dannemann, Sven & DGKJ-PO 70 & Franz, Anna & DGSPJ-PO 09 \\
\hline Dauter, Louise & DGKJ-FV 23 & Freff, Markus & DGSPJ-PO 42 \\
\hline Davies, Madhu & DGKJ-PO 50 & Frerichs, Wiebke & DGSPJ-PO 39 \\
\hline De Bock, Freia & DGSPJ-PO 28, DGSPJ-PO 43 & Frey, Urs & DGKJ-PO 67 \\
\hline de Laffolie, Jan & DGSPJ-PO 31, DGSPJ-PO 32 & Friede, Tim & DGSPJ-PO 13 \\
\hline de Sordi, Dominik & DGSPJ-PO 30 & Friedman, Mark & DGKJ-PO 45 \\
\hline Dehn- & & Friedmann, Anna & DGSPJ-PO 03 \\
\hline Hindenberg, Andrea & DGSPJ-PO 41 & Friedrich, Sebastian & DGKJ-PO 60 \\
\hline Demian, Medhat & DGKCH-FV 07 & Fröhlich-Reiterer, Elke & DGKJ-FV 17 \\
\hline
\end{tabular}




\begin{tabular}{|c|c|}
\hline Frühwald, Michael & DGKJ-PO 27 \\
\hline Fuchs, Jörg & DGKCH-PO 02 \\
\hline \multicolumn{2}{|l|}{ Fuschlberger, } \\
\hline Tamara & DGSPJ-PO 01 \\
\hline \multicolumn{2}{|l|}{$\mathbf{G}$} \\
\hline Gäbler, Niklas & DGKJ-PO 60 \\
\hline Gaiser, Ulrike & DGKJ-FV 01 \\
\hline Galler, Angela & DGKJ-FV 17 \\
\hline Garten, Lars & DGKCH-PO 11 \\
\hline Gauck, Darja & DGKJ-FV 20 \\
\hline Gebert, Lena & DGKJ-PO 02 \\
\hline Gempp, Wolfgang & DGKJ-PO 69 \\
\hline Genuneit, Jon & DGKJ-FV 16 \\
\hline \multicolumn{2}{|l|}{ Genzel-Boroviczeny, } \\
\hline Orsolya & DGKCH-P013 \\
\hline Georg, Sabine & DGSPJ-PO 28, DGSPJ-PO 43 \\
\hline Gerling, Stephan & DGKJ-FV 08 \\
\hline Gessler, Peter & DGKJ-PO 66 \\
\hline \multicolumn{2}{|l|}{ Gillesberg Lassen, } \\
\hline Sofie & DGSPJ-PO 10 \\
\hline Gindner, Lena & DGKCH-PO 10 \\
\hline Gitmans, Ulrike & DGKJ-PO 69 \\
\hline Glattacker, Manuela & DGSPJ-PO 20 \\
\hline Göbel, Ulrich & DGKJ-FV 09 \\
\hline Gödeke, Jan & DGSPJ-PO 33 \\
\hline \multicolumn{2}{|l|}{ Goldammer, } \\
\hline Stephanie & DGKJ-PO 40 \\
\hline Görig, Tatiana & DGSPJ-PO 28, DGSPJ-PO 43, \\
\hline Görsch, Melanie & DGSPJ-PO 31, DGSPJ-PO 32 \\
\hline Gosh, Arunabha & DGKJ-PO 45 \\
\hline Göttig, Stephan & DGKJ-PO 56 \\
\hline Graepler-Mainka, Ute & DGKJ-FV 28 \\
\hline Gräf, Christine & DGSPJ-PO 19 \\
\hline Grams, Daniel & DGSPJ-PO 40 \\
\hline Grasshoff, Ute & DGKJ-FV 20 \\
\hline Grillhösl, Christian & DGKCH-PO 07 \\
\hline Grimmel, Mona & DGKJ-FV 20 \\
\hline Gross, Jonathan & DGKJ-PO 30 \\
\hline \multicolumn{2}{|l|}{ Großhauser, } \\
\hline Christoph & DGKJ-FV 05 \\
\hline Grüber, Lars & DGKJ-PO 73 \\
\hline
\end{tabular}

\section{$\mathrm{H}$}

$\begin{array}{ll}\text { Haack, Tobias } & \text { DGKJ-FV 20 } \\ \text { Hackel, Katharina } & \text { DGSPJ-PO 25 } \\ \text { Hadders-Algra, Mijna } & \text { DGSPJ-FV 01 } \\ \text { Hagenguth, Andrea } & \text { DGKJ-FV 02 } \\ \text { Hagl, Beate } & \text { DGKJ-PO 27 } \\ \text { Halle, Stephan } & \text { DGKJ-FV 24 } \\ \text { Hamelmann, Eckard } & \text { DGKJ-PO 29 } \\ \text { Hansen, Gesine } & \text { DGKJ-FV 23, DGKJ-FV 24 } \\ \text { Harder, Thomas } & \text { DGSPJ-PO 11, DGSPJ-PO 12 } \\ \text { Härlein, Miriam } & \text { DGKJ-FV 13 } \\ \text { Harner, Susanne } & \text { DGKJ-PO 64 } \\ \text { Härtel, Christoph } & \text { DGKJ-PO 10 } \\ \text { Härtling, Anton } & \text { DGKJ-PO 60 } \\ \text { Hartmann, Katrin } & \text { DGKJ-FV 13 } \\ \text { Hartwig, Maite } & \text { DGSPJ-PO 21 } \\ \text { Hauch, Holger } & \text { DGKJ-FV 02, DGKJ-PO 40 } \\ \text { Hauer, Julia } & \text { DGKJ-PO 13 } \\ \text { Heckmann, Matthias } & \text { DGKJ-PO 63 } \\ \text { Hediger, Karin } & \text { DGKJ-FV 04 } \\ \text { Heidtmann, Bettina } & \text { DGSPJ-PO 42 }\end{array}$

Heimann, Alexander DGSPJ-PO 23

Heinemann,

Anna Sophie DGKJ-FV 24

Heinzelmann, Miriam DGKJ-PO 34

Heinzmann, Andrea DGKJ-PO 48

Hellmich, Martin $\quad$ DGSPJ-PO 15

Henn, Alina DGKJ-PO 44

Henrike,

Westekemper DGKJ-PO 61

Hensel, Kai O. DGKJ-PO 43

Herhold, Claudia DGSPJ-PO 06

Herrler, Angelique DGSPJ-PO 28

Hertfelder,

Hans-Jörg DGKJ-PO 16

Heuckmann, Ludger DGKJ-PO 73

Heusch, Andreas DGKJ-PO 43

Hiemisch, Andreas DGKJ-FV 16

Hilbert, Anja DGKJ-FV 16

Hilgard, Dörte $\quad$ DGKJ-FV 17

Hilgard, Dörte DGSPJ-PO 42

Hillebrand, Georg DGKJ-PO 06, DGSPJ-PO 26

Hillen, Hendrik DGSPJ-PO 15

Hinkel, Jana DGKJ-PO 52

Hippert, Felicitas DGKJ-FV 09

Hippert, Felicitas DGKJ-PO 09

Hoffmann, Vincent DGKCH-FV 01

Hoffmann,

Georg Friedrich DGKJ-FV 01

Hoffmann, Dorle $\quad$ DGSPJ-PO 43

Höhn, Cindy DGSPJ-PO 20

Holderried, Martin DGKJ-FV 28

Holl, Reinhard W. DGKJ-FV 17, DGSPJ-PO 42

Hollenbach, Sabine DGKJ-PO 39

Holstiege, Jakob DGSPJ-PO 18

Höper, Ansgar DGKJ-FV 28

Hoppe, Bernd DGKJ-PO 35

Horenkamp-

Sonntag, Dirk DGSPJ-PO 15

Horn, Denise DGKJ-PO 52

Horneff, Gerd DGSPJ-PO 29

Hörning, André DGKJ-FV 26

Hosie, Stuart DGKCH-PO 04

Hostutler, Robert DGKJ-PO 51

Huber-Lang, Markus DGKJ-PO 33

Hubertus, Jochen DGKCH-PO 06, DGKCH-

P013, DGKJ-FV 21

Hübner, Johannes DGKJ-FV 05

Hustedt, Ulf DGSPJ-FV 04, DGSPJ-FV 05

I

Inhestern, Laura DGSPJ-PO 39

Irlbauer-Müller,

Viktoria

DGSPJ-PO 02, DGSPJ-PO 04

$J$

Jaabusch, Anne DGSPJ-PO 08

Jacob, Christian DGKJ-PO 65

Jäger-Roman, Elke DGKJ-FV 07, DGKJ-PO 69

Jannes, Christiane DGSPJ-PO 15

Janssen, Niklas DGKJ-PO 31

Jansson, Annette DGKJ-FV 05

Jenke, Andreas DGKJ-PO 43

Jenke, Andreas DGSPJ-FV 02

Jit, Mark DGSPJ-PO 11

$\begin{array}{ll}\text { Johannsen, Lene } & \\ \text { Marie } & \text { DGSPJ-PO } 39 \\ \text { Jones, Simon A. } & \text { DGKJ-PO 45 } \\ \text { Jonigk, Danny } & \text { DGKJ-FV 24 } \\ \text { Jorch, Norbert } & \text { DGKJ-PO 29 } \\ \text { Jordan, Susanne } & \text { DGSPJ-PO } 35 \\ \text { Junker, Stephan } & \text { DGSPJ-PO } 37 \\ \text { Junne, Florian } & \text { DGSPJ-FV 07 } \\ \text { Jurkutat, Anne } & \text { DGKJ-FV 16 }\end{array}$

K

Kaase, Martin DGSPJ-PO 13

Kabesch, Michael DGKJ-FV 08, DGKJ-FV 18, DGKJ-PO 05, DGKJ-PO 23

Kaiser, Annette DGKCH-PO 07

Kalbitz, Miriam DGKJ-PO 33

Kallarackal, Jim DGKCH-FV 04

Kappler, Roland DGKJ-FV 21

Karsten, Katharina DGKCH-FV 01

Kaßberger, Fabian DGKJ-PO 53

Kästner, Jens DGKJ-FV 02

Kavvalou, Alexandra DGKJ-PO 14

Kerst, Gunter DGKJ-PO 11

Khasanov, Rasul DGKCH-PO 03

Kieninger, Elisabeth DGKJ-PO 67

Kiess, Wieland DGKJ-FV 16, DGKJ-PO 22, DGKJ-PO 24, DGSPJ-PO 25 DGKJ-FV 02

Kimmig, Astrid

Kiss, Natalie

Kittel, Jochen

DGKCH-FV 04, DGKCH-FV 05

Klein, Tobias

DGKJ-PO 05

DGKCH-FV 06, DGKCH-PO

10

Klemme, Mathias $\quad$ DGKJ-FV 12

Klock, Wiebke DGKJ-PO 15

Kluger, Gerhard DGKJ-FV 04

Kluger, Evelyn DGKJ-FV 04

Knauth, Burkhild DGKJ-FV 17

Knöfler, Ralf DGKJ-PO 13

Knop, Katharina $\quad$ DGKJ-PO 26, DGKJ-PO 71

Knopf, Hiltraud DGSPJ-PO 40

Knöppel, Carmen DGKJ-PO 40

Knorr, Peter

Koch, Barbara

Kollen, B.J.

Kömhoff, Martin

König, Jochem

König, Tatjana

König, Jochem

Konrad, Angelika

Kontny, Udo

DGKCH-FV 02

DGSPJ-PO 17

DGKJ-FV 27

DGKJ-PO 40

DGSPJ-PO 19

DGSPJ-PO 23

DGSPJ-PO 43

DGSPJ-PO 09

DGKJ-PO 11, DGKJ-PO 15,

DGKJ-PO 17

Koppelman, G.H. $\quad$ DGKJ-FV 27

Körner, Antje DGKJ-FV 16

Körner, Robert DGKJ-PO 32

Korten, Insa $\quad$ DGKJ-PO 67

Köstler, Ursula $\quad$ DGKJ-FV 10

Kotsias-

Konopelska, Sarah

Krägeloh-Mann,

Ingeborg

Kratz, Oliver

Krause, Laura

Krebs, Franziska

Krieb, Philipp

Krieg, Anne

Krone, Sina
DGKJ-PO 30

DGSPJ-FV 03

DGSPJ-PO 02, DGSPJ-PO 04

DGSPJ-PO 34

DGSPJ-PO 31, DGSPJ-PO 32

DGKJ-PO 66

DGSPJ-PO 30
DGSPJ-PO 22 


$\begin{array}{ll}\text { Krone, Beate } & \text { DGSPJ-PO } 42 \\ \text { Krug, Isolde } & \text { DGKJ-PO } 48 \\ \text { Kuhn, Marius } & \text { DGKJ-PO 44 } \\ \text { Kuhnert, Ronny } & \text { DGSPJ-PO 10 } \\ \text { Kuntz, Ludwig } & \text { DGSPJ-PO 15 } \\ \text { Kunz, Thomas } & \text { DGSPJ-PO 08 } \\ \text { Kurth, Ingo } & \text { DGKJ-PO 18 } \\ \text { Küster, Helmut } & \text { DGSPJ-PO 13 } \\ \text { Kuttumuratova, } & \\ \text { Aigul } & \text { DGSPJ-PO 14 }\end{array}$

\section{L}

Laaß, Martin W.

Lacher, Martin

Lange, Anja

Lange, Martina

Lange, Cornelia

Lange, Karin

Langer, Melissa

Langer, Thorsten

Langer, Thorsten

Längler, Alfred

Lassay, Lisa

Latzin, Philipp

Lau, Susanne

Laugwitz, Lucia

Laurenz, Maren

Lauten, Melchior

Lehmann, Franziska

Lehmann-Kannt,

Stephanie

Leitzen, Sarah

Leiz, Steffen

Lewien, Christiane

Liapi, Maria

Licata-Dandel, Maria

Liebl, Bernhard

Liese, Johannes G.

Lilienthal, Eggert

Linnemann, Knud

Lipphaus, Andreas

Lippmann, Norman

Lisec, Kristina

Lohmar, Susanne

Lorenz, Laura

Lücke, Thomas

Lüders, Anja

Ludwig, Hans

Christoph

Ludwikowski,

Barbara

DGKJ-PO 07

DGKCH-PO 08

DGKJ-PO 63

DGSPJ-PO 13

DGSPJ-PO 35

DGSPJ-PO 41

DGKJ-FV 24

DGSPJ-PO 17

DGSPJ-PO 38

DGKJ-FV 09

DGKJ-PO 11, DGKJ-PO 15,

DGKJ-PO 17, DGKJ-PO 18

DGKJ-PO 67

DGKJ-PO 30

DGKJ-FV 20

DGKJ-PO 65

DGKJ-PO 10

DGSPJ-PO 35

DGSPJ-PO 07

DGSPJ-PO 16

DGKJ-PO 62

DGKJ-PO 22

DGKJ-PO 29

DGSPJ-PO 03

DGKJ-FV 14

DGKJ-FV 13

DGSPJ-PO 42

DGKJ-PO 63

DGKCH-PO 09

DGKJ-PO 24

DGKJ-FV 21

DGKJ-PO 04

DGSPJ-PO 34

DGKJ-PO 01

DGKJ-FV 14

DGKJ-PO 61

DGKCH-FV 03

\section{$M$}

Mack-Detlefsen,

Birte

DGKCH-FV 07, DGKCH-PO

10

Maier, Pia

DGSPJ-PO 14

Mall, Volker

Malonga Makosi,

Dorothée

DGSPJ-PO 03

Malota, Mark

Manuela, Richter

DGSPJ-PO 19

DGKCH-PO 04

DGKJ-FV 24

DGKJ-PO 71
Marquardt, Thorsten DGKJ-PO 08

Marsden, Deborah DGKJ-PO 46, DGKJ-PO 51

Martignoni, Kathrin DGSPJ-PO 24

Märzheuser, Stefanie DGKCH-FV 01

Maurer, Kristina DGKCH-PO 03

Mayle, Paulina DGKCH-PO13

Meier, Stephanie DGKJ-FV 25

Meier-Sprinz, Brigitte DGKJ-FV 03

Meigen, Christof DGKJ-PO 22

Meinel, Jörn DGKJ-PO 13

Meißner, Moritz DGKJ-PO 04

Meitinger, Thomas DGKJ-PO 27

Melter, Michael DGKJ-FV 08, DGKJ-FV 18,

DGKJ-PO 05, DGKJ-PO 23

Mendonca, Marina DGKJ-FV 11

Mense, Lars DGKJ-PO 54, DGSPJ-FV 08

Merklein, Domenica DGKCH-FV 07

Metzner, Gloria DGSPJ-PO 20

Mey, Marie Elisa DGSPJ-FV 02

Meyer-Borgstädt,

Anna Katharina

Michel, Holger

DGSPJ-PO 09, DGSPJ-PO 24

Mierke, Franz

DGKJ-FV 08

Mildenberger, Philipp DGSPJ-PO 19

Minden, Kirsten DGSPJ-PO 29

Minderjahn,

Maximiliane

Moebus, Ralf

DGKCH-FV 04, DGKCH-FV 05

Mohn, Josephine

DGKJ-PO 68

Mönkemöller, Kirsten DGSPJ-PO 42

Moser, Olga

DGKJ-PO 11

Mueller, Alfred

DGKCH-PO 01, DGKJ-FV 19 , DGKJ-PO 19

Muensterer, Oliver

DGKJ-PO 21, DGSPJ-PO 23,

DGSPJ-PO 33

Mühlbacher, Tobias DGKJ-FV 22

Müller, Dominik

DGKJ-PO 38

Müller, Andreas

DGKJ-PO 55

Müller, Christoph

Müller, Christine

Müller, Andreas

Müller-Godeffroy,

Esther

DGKJ-PO 60

DGKJ-PO 71

DGSPJ-PO 15

DGSPJ-PO 42

$\mathrm{N}$

Nagel, Ann-Kathrin DGKJ-PO 35

Naust, Barbara DGKJ-PO 06

Nawabi, Farah DGSPJ-PO 34

Neininger,

Martina Patrizia DGSPJ-PO 25

Nennstiel, Uta

DGKJ-FV 14

Neubert, Antje

DGKJ-FV 15, DGKJ-FV 26, DGSPJ-PO 16, DGSPJ-PO 19

Neuhauser,

Hannelore

Niemeyer, C

DGSPJ-PO 22

Niessner, Claudia DGKJ-PO 34

Nissen, Matthias DGKCH-PO 05

Nitsche, Catharina DGKJ-FV 27

Nitzschke, Nikola DGSPJ-PO 27

Nonnenmacher, Lena DGKJ-PO 05

Notheis, Gundula $\quad$ DGKJ-PO 27
0

Obermeier, Viola $\quad$ DGKJ-FV 12

Oetzmann von

Sochaczewski,

Christina

DGKJ-PO 21

Omran, Heymut DGKJ-PO 25

Ost, Michael DGKJ-PO 09

Osten, Heimke DGKJ-PO 63

$P$

Pagel, Julia $\quad$ DGKJ-PO 10

Papaioannou, Kyriaki DGKJ-PO 11

Pappa, Angeliki DGKJ-PO 17

Pappas, Angeliki DGKJ-PO 15

Papsch, Matthias DGSPJ-PO 42

Paul, Thomas DGSPJ-PO 13

Pauly, Marcus DGKJ-PO 04

Pawils, Silke DGSPJ-PO 27

Peter, Corinna DGKJ-FV 23

Peters, Anthea DGKJ-PO 35, DGKJ-PO 70

Peters, Jochen DGKJ-PO 62

Pfeifer, Sandra DGKJ-FV 24

Pfister, Marc DGKJ-PO 37

Philippi, Heike DGSPJ-FV 01, DGSPJ-PO 28,

DGSPJ-PO 43

Piepenbrink, Lea DGKJ-PO 06

Pietz, Joachim DGSPJ-FV 01

Pirr, Sabine

Poethko-Müller,

Christina

Pohl, Michael

DGKJ-FV 23, DGKJ-FV 24

Pohlmann, Ulrich

DGSPJ-PO 10, DGSPJ-PO 40

DGKJ-PO 39

DGKJ-PO 11

Poklekowski, Sonja DGSPJ-PO 13

Poralla, Silvia

Porst, Patricia

DGKJ-PO 55

DGSPJ-PO 36

Potapow, Antonia DGKJ-FV 08

Pothmann, Raymund DGSPJ-PO 21

Poulain, Tanja

Pozo Martin,

Francisco

Preßmar, Jochen

DGKJ-FV 16, DGKJ-PO 22

Prifert, Christiane

DGSPJ-PO 11, DGSPJ-PO 12

Promm, Martin

DGKJ-PO 33

Proquitté, Hans

DGKJ-FV 13

DGKJ-FV 18

DGSPJ-FV 08

Q

Quirke, Jo DGKJ-PO 50

$\mathrm{R}$

Radke, Michael DGSPJ-PO 31, DGSPJ-PO 32

Raecke, Olaf DGKJ-PO 02

Rakete, Stefan DGKJ-PO 62

Rall, Katharina

DGKCH-PO 02

Ramsey, Kathryn

DGKJ-PO 67

Rascher, Wolfgang DGKJ-FV 15, DGKJ-FV 26,

DGSPJ-PO 19

Rawolle, Tanja DGKCH-FV 05

Reibel, Nora Johanna DGKJ-FV 22

Reichert, Jörg DGSPJ-FV 08

Reinhardt, D DGSPJ-PO 17

Reismann, Josephine DGKCH-FV 04, DGKCH-FV 05

Reismann, Marc

Renner, Ellen

DGKJ-PO 27 


\begin{tabular}{|c|c|c|c|c|c|}
\hline Reschke, Madlen & DGKJ-PO 16, DGKJ-PO 61 & Schönfeld, Annika & DGKJ-PO 24 & Sturm, Marc & DGKJ-FV 20 \\
\hline Rieß, Olaf & DGKJ-FV 20 & Schönfeld, Viktoria & DGSPJ-PO 11, DGSPJ-PO 12 & Sturm, Ekkehard & DGKJ-FV 28 \\
\hline Ritter, Jessica & DGKJ-FV 21 & Schriegel, Felix & DGKJ-PO 63 & Sulser, Claudia & DGSPJ-PO 24 \\
\hline $\begin{array}{l}\text { Roche, Franziska } \\
\text { Romualdi, }\end{array}$ & DGKJ-PO 55 & $\begin{array}{l}\text { Schriek, Katharina } \\
\text { Schröder, }\end{array}$ & DGKCH-FV 03 & & \\
\hline Alessandro & DGKCH-FV 04 & Jenyffer-Tabea & DGKCH-PO 11 & $\mathrm{~T}$ & \\
\hline Rösch, Wolfgang $\mathrm{H}$. & DGKJ-FV 18 & Schröder, Tabea & DGKCH-PO 12 & Tacke, Uta Hedwig & DGSPJ-FV 01 \\
\hline Roskopf, Markus & DGKJ-PO 43 & Schröder, Arne & DGKJ-PO 21 & Taylan, Christina & DGKJ-PO 38 \\
\hline Ross, Richard John M & DGKJ-PO 50 & Schröpf, Sebastian & DGKCH-P013 & Tenbrock, Klaus & DGSPJ-PO 29 \\
\hline Roth, Johannes & DGKJ-FV 23 & Schubert, Markus & DGKJ-PO 66 & Terzi, Alexandra & DGKJ-PO 14 \\
\hline Roth, Michèle & DGKJ-PO 20 & Schulz, Bettina & DGKJ-PO 39 & Teufel-Schäfer, Ulrike & DGKJ-PO 60 \\
\hline \multirow[t]{2}{*}{ Rothe, Karin } & DGKCH-FV 05, DGKCH-PO & Schulz, Maike & DGSPJ-PO 18 & Thamm, Roma & DGSPJ-PO 22 \\
\hline & 11, DGKCH-PO 12 & Schulze, Gisela & DGSPJ-PO 30 & Thiels, Charlotte & DGKJ-PO 01 \\
\hline Rothenfusser, Simon & DGKJ-PO 27 & Schumacher, Pauline & DGKJ-PO 26 & Thome, Ulrich & DGKCH-PO 08 \\
\hline Rüdiger, Mario & DGKJ-PO 54, DGSPJ-FV 08 & Schumacher, Andrea & DGSPJ-PO 42 & Thöne, Kathrin & DGSPJ-PO 11 \\
\hline Rudin, Christoph & DGKJ-PO 20 & Schuster, Tobias & DGKCH-FV 02 & Thumfart, Julia & DGKJ-PO 38 \\
\hline \multirow[t]{3}{*}{ Rupp, Jan } & DGKJ-PO 10 & Schuster, Volker & DGKJ-PO 61 & Tillmann, Roland & DGKJ-FV 06 \\
\hline & & Schwab, Karl Otfried & DGSPJ-PO 42 & Timmer, Antje & DGSPJ-PO 30 \\
\hline & & Schwartz, Oliver & DGKJ-PO 25 & Timpe, Felix & DGSPJ-PO 08 \\
\hline S & & Schweiger, & & Tittel, Sascha R. & DGKJ-FV 17 \\
\hline Sachers, Julia & DGKJ-FV 04 & Heidemarie & DGSPJ-PO 42 & Toni, Irmgard & DGKJ-FV 15, DGSPJ-PO 19, \\
\hline Sachs, Bernhardt & DGSPJ-PO 16 & Schweiger- & & & DGSPJ-PO 16 \\
\hline Samel, Christina & DGSPJ-PO 15 & Kabesch, Andrea & DGKJ-PO 64 & Traidl-Hoffmann, & \\
\hline Sansone, Kenneth J & DGKJ-PO 51 & Schweinitz, Dietrich & DGKJ-FV 21 & Claudia & DGKJ-PO 27 \\
\hline Sarganas, Giselle & DGSPJ-PO 22, DGSPJ-PO 40 & Schwerdtfeger, & & Trenkel, Stefan & DGSPJ-PO 31 \\
\hline Sarif, Frieda & DGKJ-PO 08 & Cristina & DGKJ-PO 04 & Trenkel, Stefan & DGSPJ-PO 32 \\
\hline Schacht, Sabine & DGKJ-PO 15, DGKJ-PO 17 & Seebauer, Ludwig & DGKJ-PO 64 & Trepels-Kottek, & \\
\hline Schad, Martina & DGKCH-FV 04 & Seefried, Lothar & DGKJ-PO 59 & Sonja & DGKJ-PO 11 \\
\hline Schäfer, & & Seelbach-Göbel & Birgit DGKJ-PO 05, & Treskova, Marina & DGSPJ-PO 11, DGSPJ-PO 12 \\
\hline Frank-Mattias & DGKCH-PO 07 & & DGKJ-FV 08, DGKJ-FV 18, & Tretiakowa, Irina & DGKJ-FV 02 \\
\hline Schäfer, & & & DGKJ-PO 23 & Tröbs, Ralf-Bodo & DGKCH-PO 05 \\
\hline Rebecca Nathalie & DGKJ-PO 52 & Seiffert, Peter & DGKJ-PO 31 & Ralf-Bodo & DGKCH-PO 09, DGKJ-PO 31 \\
\hline Schaffer, Annika & DGKJ-PO 33 & Seipolt, Barbara & DGKJ-PO 54 & Trollmann, Regina & DGKJ-FV 15, DGKJ-FV 26 \\
\hline Scharenberg, & & Siaplaouras, Jannos & DGKJ-PO 34 & Tsaka, Sofia & DGKJ-PO 14 \\
\hline Swantje & DGSPJ-PO 23 & Sichardt, Lucas & DGKJ-PO 40 & Tsokas, Jean & DGKJ-PO 73 \\
\hline Schatz, Ulrich A & DGKJ-PO 27 & Siedler, Anette & DGSPJ-PO 10 & & \\
\hline Schäuble, & & Siefert, Sönke & DGSPJ-PO 27 & & \\
\hline Julian Norton & DGKJ-PO 41 & Siegmund, Mira & DGKJ-PO 10 & U & \\
\hline Schaudeck, Martina & DGKJ-FV 04 & Siegmund, Cornelia & DGSPJ-PO 36 & Unkel, Steffen & \\
\hline Scheer, Mario & DGKJ-FV 02 & Simma, Alexander & DGKJ-FV 08 & nno & DGKJ-FV 24 \\
\hline Scheithauer, Simone & DGSPJ-PO 13 & Sinnig, Mechthild & DGKCH-FV 03 & Urlichs, Florian & DGKJ-PO 71 \\
\hline Schiffner-Rohe, Julia & DGKJ-PO 65 & Sohrabi, Keywan & DGSPJ-PO 31, DGSPJ-PO 32 & Urschitz, & \\
\hline Schlack, Robert & DGSPJ-PO 37 & Sommer, Julian & DGKJ-PO 56 & Michael Siegfried & DGSPJ-PO 19, DGSPJ-PO 43 \\
\hline Schleußner, Ekkehard & DGSPJ-FV 08 & Sorg, Anna-Lisa & DGKJ-FV 12 & Utzig, Norbert & DGKJ-PO 63 \\
\hline Schley, Katharina & DGKJ-PO 65 & Spiecker, Indra & DGSPJ-PO 15 & & \\
\hline Schlößer, Rolf & DGKJ-PO 56 & Spiegler, Juliane & DGKJ-FV 11 & & \\
\hline Schmidt, Yannik & DGKCH-PO 06 & Spielberger, & & & \\
\hline Schmidt, Beatrix & DGKJ-PO 30 & Benedikt D & DGKJ-PO 27 & & \\
\hline Schmidt- & & Sprikkelman, A.B. & DGKJ-FV 27 & Vaillant, Vera & DGKJ-FV 02 \\
\hline Choudhury, Anjona & DGKJ-PO 01 & Spycher, Ben & DGKJ-PO 67 & van den Anker, & \\
\hline Schmitt, Jochen & DGSPJ-FV 08 & Staab, Doris & DGKJ-PO 30 & Johannes N. & DGKJ-PO 37 \\
\hline Schneider, & & Stäbler, Caroline & DGKJ-PO 42 & van den Berg, Nanja & DGSPJ-PO 31, DGSPJ-PO 32 \\
\hline DominikThomas & DGKJ-FV 09 & Stanulla, Martin & DGKJ-FV 23 & van den Heuvel, & \\
\hline Schneider, Hendryk & DGKJ-PO 03 & Stapel, Matthias & DGSPJ-PO 20 & & DGKJ-PO 25 \\
\hline Schneider, D.T. & DGKJ-PO 09 & Steffen, Annika & DGSPJ-PO 18 & van Donge, Tamara & DGKJ-PO 37 \\
\hline Schneider, Florian & DGKJ-PO 26, DGKJ-PO 71, & Stehr, Maximilian & DGKCH-PO 07 & van Heek, Ralf & DGSPJ-PO 26 \\
\hline & DGKJ-PO 73 & Stein, Alexander & DGSPJ-PO 17 & van Kuijck, Marcel & DGKJ-PO 51 \\
\hline Schneider, Udo & DGSPJ-PO 11 & Steinau, Gerd & DGKJ-PO 15 & Varnaccia, Gianni & DGSPJ-PO 35 \\
\hline Schneider, Nicolas & DGSPJ-PO 31 & Stingl, Julia & DGSPJ-PO 16 & Vazquez-Jimenez, & \\
\hline Schneider, Henning & DGSPJ-PO 31 & Stock, Stephanie & DGSPJ-PO 34 & Jaime F. & DGKJ-PO 11 \\
\hline Schneider, Nicolas & DGSPJ-PO 32 & Stockklausner, & & Viebahn, Richard & DGKJ-PO 31 \\
\hline Schneider, Henning & DGSPJ-PO 32 & Clemens & DGKJ-PO 19 & Viemann, Dorothee & DGKJ-FV 23, DGKJ-FV 24 \\
\hline Schnopp, Nina & DGKJ-PO 27 & Stöhrer, Stephan & DGKJ-PO 04 & Vieten, Gertrud & DGKJ-FV 24 \\
\hline Schoberer, Mark & $\begin{array}{l}\text { DGKJ-PU } 21 \\
\text { DGKJ-PO } 11\end{array}$ & rch Katia & DGKJ-PO 12 & Vijay, Suresh & DGKJ-PO 45 \\
\hline Scholten, Nadine & & & DGKLEV12 & Vlajnic, Dejan & DGKJ-PO 70 \\
\hline & & Streng, Andrea & DGKJ-FV I3 & Vockley, Jerry & DGKJ-PO 46 \\
\hline Scholz, Tasja & DGKJ-PO 10 & Stuber, Felicitas & DGSPJ-FV 07 & Voet, Bernard & DGKJ-PO 50 \\
\hline cholz, Stefan & DGSPJ-PO 11, DGSPJ-PO 12 & Study Group, CEDAT/ & DGSPJ-PO 31, DGSPJ-PO 32 & & \\
\hline
\end{tabular}




Vogel, Mandy
Vogel, Cornelia
Vogl, Thomas
Vokuhl, Christian
Völlger, Lena
von Bernuth, Horst
von Bonin, Malte
von Both, Ulrich
von Eiff, Christof
von Kaisenberg,
Constantin
von Köckritz-
Blickwede, Maren
von Kries, Rüdiger
von Scheidt, Fabian
von Schnakenburg,
Christian
von Schweinitz,
Dietrich

Dietrich

W

Wagner, Richard

Wagner, Norbert

Wahler, Pauline

Wahler, Steffen

Wahler, Elena

Waldeck, Edith

Walter, Irmgard

Weber, Lutz

Weber, Stefanie

Weber, Martin Willi

Wechsler, Christoph

Weis, Meike

Weiß-Becker,

Christoph

Weißbrich, Benedikt

Welzel, Tatjana

Wendeborn, Mathias

Wendling, Danielle

Weniger, Max

Wenzel, Maren

Wessel, Lucas

Westerlaken-

van Ginkel, C.D.

Weyersberg, Annic

Wichelhaus, Thomas

Wichmann, Ole

Wilke, Lucia

Willems, Jana

Willers, Maike

Wimmer, Stefan

Witowski, Andrea

Wittekindt, Boris

Witzel, Ulrich

Wobbe-Ribinski,

Stefanie

Wohlrab, Doris

Wolf, Christian

Wolf, Johannes

Wolke, Dieter

Wölke, Christian

Woopen, Christiane

Wördehoff, Rosa

Wörner, Andreas

Wurm, Michael
DGKJ-FV 16

DGKJ-PO 53

DGKJ-FV 23

DGKJ-FV 09

DGKJ-FV 24

DGKJ-PO 30

DGKJ-PO 13

DGKJ-FV 05

DGKJ-PO 65

DGKJ-FV 24

DGKJ-FV 24

DGKJ-FV 12

DGKJ-FV 25

DGKJ-PO 02

DGKCH-PO 06

DGKCH-PO 08

DGKJ-PO 17, DGKJ-PO 18

DGKCH-PO 01

DGKCH-PO 01, DGKJ-FV 19,

DGKJ-PO 19, DGKJ-PO 59

DGKJ-FV 19

DGSPJ-PO 20

DGKJ-PO 66

DGKJ-PO 38

DGKJ-PO 40

DGSPJ-PO 14

DGSPJ-PO 23

DGKCH-PO 03

DGKJ-PO 69, DGSPJ-PO 26

DGKJ-FV 13

DGKJ-PO 20, DGKJ-PO 37

DGKJ-FV 05

DGKCH-PO 06

DGSPJ-PO 36

DGKJ-PO 44

DGKCH-PO 03

DGKJ-FV 27

DGKJ-FV 10

DGKJ-PO 56

DGSPJ-PO 11, DGSPJ-PO 12

DGKJ-PO 43

DGSPJ-PO 38

DGKJ-FV 24

DGKJ-FV 15

DGKJ-FV 09

DGKJ-PO 56

DGKCH-PO 09

DGSPJ-PO 15

DGSPJ-PO 24

DGKJ-FV 28

DGSPJ-PO 42

DGKJ-FV 11

DGKJ-PO 56

DGKJ-FV 10, DGSPJ-PO 15

DGKJ-PO 06

DGKJ-PO 20

DGKJ-FV 17, DGKJ-PO 64
Wygoda, Simone

Wygold, Thorsten

DGKJ-PO 39

DGSPJ-PO 08

Z

Zahn, Julia $\quad$ DGKJ-FV 26

Zeiher, Johannes $\quad$ DGSPJ-PO 35

Zeißig, Yvonne DGKJ-PO 07

Zemlin, Michael $\quad$ DGSPJ-PO 07

Zimmer, Klaus-Peter DGSPJ-PO 31, DGSPJ-PO 32

Zipfel, Stephan DGSPJ-FV 07

Zirn, Birgit DGKJ-PO 44 